Lehrstuhl für Apparate- und Anlagenbau

Experimentelle Spannungsanalyse

der Technischen Universität München

\title{
Entwicklung eines numerischen Berechnungsverfahrens für Rührwerksschwingungen
}

\author{
Thomas Peter Michael Berger \\ Vollständiger Abdruck der von der Fakultät für Maschinenwesen \\ der Technischen Universität München zur Erlangung \\ des akademischen Grades eines \\ Doktor-Ingenieurs \\ genehmigten Dissertation.
}

Vorsitzender: $\quad$ Univ.-Prof. Dr.-Ing. D. Weuster-Botz

Prüfer der Dissertation:

1. Univ.-Prof. Dr.-Ing. K. Strohmeier

2. Univ.-Prof. Dr.-Ing., Dr.-Ing. habil. J. Stichlmair

Die Dissertation wurde am 16.06.2004 bei der Technischen Universität München eingereicht und durch die Fakultät für Maschinenwesen am 11.01.2005 angenommen. 



\section{Vorwort}

Die vorliegende Arbeit entstand während meiner Tätigkeit als wissenschaftlicher Assistent am Lehrstuhl für Apparate- und Anlagenbau - Experimentelle Spannungsanalyse der Technischen Universität München.

Meinem Doktorvater Ordinarius Univ.-Prof. Dr.-Ing. Klaus Strohmeier danke ich ganz besonders für die Möglichkeit zur Durchführung der Arbeit und das fachliche Wissen, welches er mir in den letzten Jahren der Zusammenarbeit vermittelt hat.

Seine Anregungen und Ratschläge auf der einen, aber auch die Gewährung nötiger Freiräume auf der anderen Seite, waren ideale Voraussetzungen dafür, dass diese Arbeit eine „runde Sache“ wurde. Danke für Ihr stetiges Interesse an meiner Arbeit, Ihre moralische Unterstützung und dass Sie immer ein „offenes Ohr“ hatten!

Herrn Univ.-Prof. Dr.-Ing., Dr.-Ing. habil. Stichlmair danke ich für die Übernahme des Korreferats, Herrn Univ.-Prof. Dr.-Ing. Weuster-Botz für die Übernahme des Prüfungsvorsitzes.

Meinen Industriepartnern Dipl-Ing. Detlev Huber und ganz besonders Dipl.-Ing. Walter Schwechheimer von der Firma Fluid Misch- und Dispergiertechnik GmbH gilt großer Dank für die Bereitstellung des Versuchsrührwerks und ihre ausgezeichneten fachlichen Hilfestellungen. Danke, dass Ihr von Anfang an dabei gewesen seid!

Den Herren Dr.-Ing. Reinhard Geisler und Dr.-Ing. Achim Knoch danke ich für ihre fachlichen Ratschläge auf diversen Fachtagungen.

Ich danke allen Kollegen am Lehrstuhl für Apparate- und Anlagenbau der TU München für das gute und freundschaftliche Arbeitsklima.

Meinem Kollegen Dipl.-Ing. Christoph Reichel danke ich besonders für die hilfreichen fachlichen Diskussionen im In- und Ausland und die Durchsicht des Manuskripts.

Den Angehörigen der lehrstuhleigenen Werkstatt danke ich für Ihre Unterstützung beim Aufbau und Betreiben des Versuchsstands. 
Mein großer Dank gilt allen Studenten, die durch Diplom-, Semesterarbeiten und HiWi-Tätigkeiten einen wichtigen Beitrag zum Gelingen dieser Arbeit geleistet haben. Insbesondere möchte ich mich beim meinen beiden ehemaligen "Spitzen-Studis“ Dipl.-Ing. Bernhard Eckl und Dipl.-Ing. Johannes Ametsbichler für ihr herausragendes Engagement bedanken.

Ich danke Rudi Hillreiner und Jutta Hofmann für die Durchsicht des Manuskripts.

Nicht zuletzt gilt schließlich mein ganz besonderer Dank meiner Freundin Doerthe, die immer hinter mir stand.

Die Jahre am LAA werden mir stets in bester Erinnerung bleiben!

München, im Februar 2005 



\section{Inhalt}

1 Einleitung 1

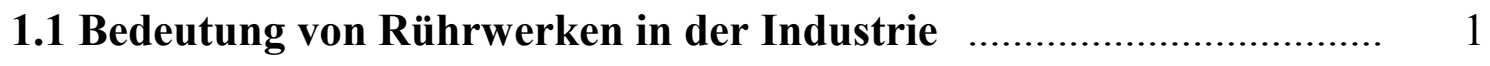

1.2 Rührwerksschwingungen .......................................................... 2

1.2.1 Betrieb im Leerlauf (Luft) ........................................................ 4

1.2.2 Betrieb im Fluid .................................................................... 6

1.3 Bisherige Lösungsansätze ............................................................ 8

1.3.1 Analytische Ansätze für den Betrieb im Leerlauf ......................... 8

1.3.2 Analytische Erweiterungen für den Betrieb im Fluid ..................... 9

1.3.3 Numerische Ansätze ............................................................. 12

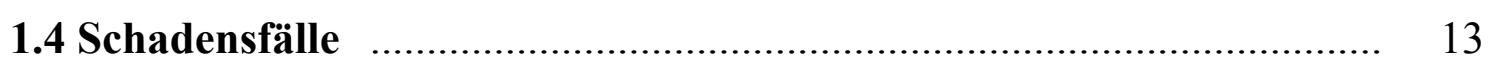

1.5 Ziel der vorliegenden Arbeit ........................................................ 16

1.6 Gliederung der Arbeit $\quad$................................................................ 17

2 Theoretischer Grundlagenteil $\quad 19$

2.1 Mechanik des Einmassenschwingers $\quad$............................................. 19

2.2 Kontinuierlicher Schwinger f.......................................................... 22

2.3 Grundlagen der Rotordynamik ................................................... 27

2.3.1 Unwuchtanregung ................................................................ 27

2.3.2 Fluidanregung ........................................................................... 35

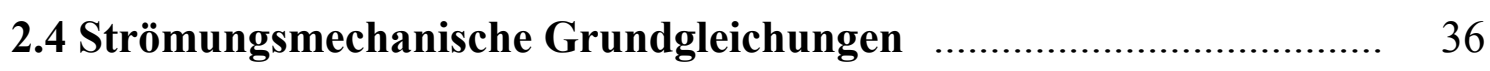

2.4.1 Massenbilanz ........................................................................ 36

2.4.2 Impulsbilanz ....................................................................... 36

2.4.3 Allgemeine Form der Transportgleichungen ............................... 37

2.4.4 Berechnung turbulenter Strömungen .......................................... 38

2.5 Einfluss des Fluids auf die Biegeschwingungen von Rührwerken ..... 40

2.5.1 Fluiddynamische Zusatzmasse ................................................ 40

2.5.2 Fluiddämpfung $\quad$....................................................................... 41

2.5.3 Quertriebskraft .................................................................... 42

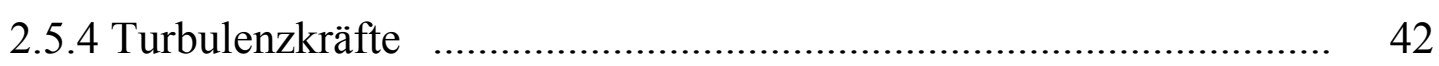

2.6 Einfluss der Einspannsteifigkeit auf das Biegeschwingungsverhalten von Rührwerken ...................................................................... 47 


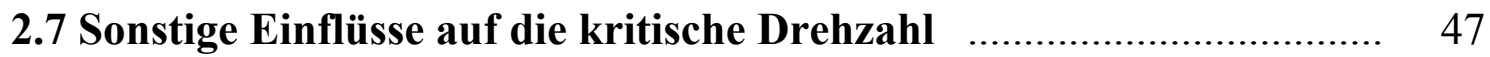

2.7.1 Kreiseleffekt ...................................................................... 47

2.7.2 Axialkräfte ........................................................................ 47

2.7.3 Schwingung aufgrund von Wälzlageranregung ............................ 48

\section{Numerisches Berechnungsverfahren $\quad 50$}

3.1 Diskretisierung der strömungsmechanischen Grundgleichungen $\ldots . . \quad 50$

3.1.1 Berechnungsgitter ................................................................. 50

3.1.2 Zeitschrittweite und Iterationszahl f........................................... 50

3.1.3 Räumliche Diskretisierung ..................................................... 51

Finite-Volumen Methode ........................................................... 51

Forderung an die Diskretisierung der Transportgleichung ............ 53

3.1.4 Turbulenzmodellierung ............................................................. 54

k- $\varepsilon$ Turbulenzmodell ................................................................... 55

RNG k-EModell ........................................................................ 56

Reynolds-Spannung- Modelle ................................................. 57

Randbedingungen für turbulente Strömungen ............................. 58

3.1.5 Numerischer Lösungsalgorithmus ............................................ 59

3.2 Simulation der strukturmechanischen Rührerdynamik $\ldots \ldots \ldots \ldots \ldots \ldots \ldots \ldots . . . .60$

3.2.1 Numerisches Ersatzmodell der Rührerstruktur ............................ 60

Darstellung der Bewegungsgleichungen .................................... 60

Modellierung von Steifigkeit und Dämpfung .............................. 60

Modellierung der äußeren Lasten ................................................ 63

Modellierung der Bewegungsgleichungen .................................. 64

Zeitliche Diskretisierung der Struktur ........................................... 65

3.2.2 Modellierung der Rührerwelle als Euler-Bernoulli-Balken ............ 70

Räumliche Diskretisierung .................................................. $\quad 70$

Zeitliche Diskretisierung und iterative Lösung des

Gleichungssystems ..................................................................... 72

3.2.3 Kopplung von Strömung und Struktur ...................................... 74

Realisierung der Gitterverschiebung .......................................... 74

Zurücksetzen und Verdrehen des Rührwerks ................................ 74

Aufsetzen einer neuen Verschiebung im CFD-CODE ................... 75

Auslesen der Strömungskräfte ................................................... 78 
Berechnung der Auslenkung des Rührwerks ............................. 79

Aufgabe von Wandgeschwindigkeiten ....................................... 80

Festlegung der Zeitschrittweite ............................................... 80

3.2.4 Testrechnungen und Validierung des numerischen Ersatzmodells .... 81

Testrechnungen im Leerlauf ................................................... 81

Validierung des numerischen Ersatzmodells im Leerlauf .............. 85

Parameterstudien im Fluid (Wasser) .......................................... 87

Vergleich des numerischen Ersatzmodells mit dem numerischen Balkenmodell ........................................................................ 91

3.2.5 Berücksichtigung der Einspannsteifigkeit des Rührwerks $\quad$............. 92

Belastungsfall Biegung des Einspannbalkens ........................... 92

Belastungsfall Torsion des Einspannbalkens .............................. 96

Ersatzmodell für das System Rührwerk-Einspannträger ................ 97

Vierkantprofil ........................................................................... 97

3.3 Zusammenfassung: Berechnungsverfahren $\quad$.................................. 100

4 Experimentelle Untersuchungen $\quad 101$

4.1 Beschreibung der Versuchseinrichtung und

Versuchsdurchführung ............................................................. 101

4.1.1 Versuchsstandübersicht …....................................................... 101

4.1.2 Versuchstechnik und Durchführung .......................................... 105

Leistungseintrag des Rührwerks ................................................ 105

Geschwindigkeitsmessung des Strömungsfelds mit LDA ............... 106

Messung der Rührwerksschwingungen ..................................... 112

Druckmessungen an den Rührerblättern ................................... 118

4.2 Experimentelle Ergebnisse im Leerlauf (Luft) ............................... 122

4.2.1 Bestimmung der Eigenfrequenz und der Materialdämpfung ......... 122

4.2.2 Bestimmung der Exzentrizität ............................................... 122

5 Berechnungsbeispiele $\quad 125$

5.1 Vergleich des numerischen Ersatzmodells mit experimentellen

Ergebnissen $\quad$............................................................................ 125

5.1.1 Schrägblattrührer und $\mathrm{F} 1^{\odot}{ }^{\odot}$-Rührer im Leerlauf (Luft) ................... 125 
Schrägblattrührer im Leerlauf ..................................................... 127

$F 1^{\circledR}$-Rührer im Leerlauf ......................................................... 130

5.1.2 Schrägblattrührer und $\mathrm{F} 1^{\odot}$-Rührer in Fluid (Wasser) ................... 133

Validierung des numerischen Strömungsfelds ............................ 134

Schrägblattrührer im Wasser .................................................... 136

$F 1^{\odot}$-Rührer im Wasser ........................................................... 137

5.2 Einfluss verschiedener Fluide auf das Frequenzverhalten

des Rührwerks ....................................................................... 139

5.2.1 Einfluss des Fluids auf die kritische Drehzahl und die

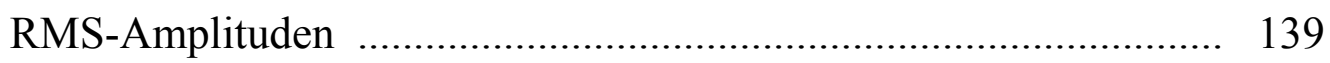

5.2.2 Fluidinduzierte Schwingungen im unterkritischen Drehzahlbereich . 140

\subsection{Einfluss der Einspannsteifigkeit auf das Frequenzverhalten}

des Rührwerks ....................................................................... 146

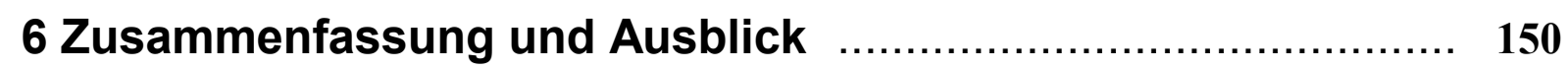

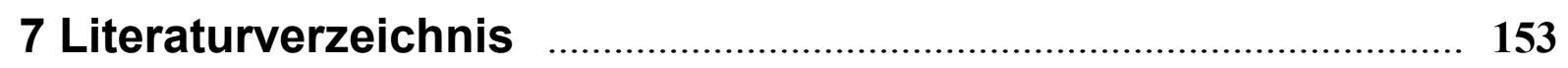




\section{Nomenklatur}

\section{Lateinische Symbole}

A

A

$A_{B}$

$A_{R}$

$\mathrm{B}$

$b_{1}$

$b_{2}$

$c$

$c_{F}$

$c_{Q}$

$c_{P}$

$c_{t}$

$c_{\Gamma}$

C

C

$D$

$D$

$D_{R, i}$

$D_{R, a}$

$d$

$d_{e-2}$

$d_{I}$

$d_{\text {Rührer }}$

$d_{\text {Welle }}$

E

$f$

$f_{1}$

$f_{B}$

$f_{D}$

F

$F_{a x}$

Fläche

Konstante

Fläche des Rührerblattes

seitliche Projektion des Rührorgans

Breite

Außenkantenlänge Vierkantträger

Innenkantenlänge Vierkantträger

Federsteifigkeit

Kraftbeiwert Fluid

Quertriebsbeiwert

Druckkraftbeiwert

Tangentialkraftbeiwert

Zirkulationsbeiwert

Dämpfungsmatrix

Konstante

Durchmesser des Rührbehälters

Lehr'sches Dämpfungsmaß

Innendurchmesser Lager

Außendurchmesser Lager

Durchmesser

Durchmesser Laserstrahlen

Abstand Interferenzstreifen

Durchmesser Rührerkopf

Durchmesser Welle

Elastizitätsmodul

Frequenz

1. Eigenfrequenz

Blattfrequenz

Dopplerfrequenz

Kraft

: $\quad$ Fluidkraft in axialer Richtung 


$\begin{array}{lll}F_{\text {Blatt, res }} & : & \text { resultierende Druckkraft auf das Rührerblatt } \\ F_{d} & : & \text { Fluiddämpfungskraft } \\ F_{\text {Dämpfung }} & : & \text { Materialdämpfungskraft } \\ F_{\text {elast }} & : & \text { elastische Rückstellkraft } \\ F_{G} & : & \text { Gewichtskraft } \\ F_{h} & : & \text { hydraulische Kraft } \\ F_{\text {krit }} & : & \text { kritische Axialkraft } \\ F_{r, \text { Fluid }} & : & \text { radiale Fluidkraft } \\ F_{r, \text { Luft }} & : & \text { radiale Kraft im Leerlauf } \\ F_{\text {Rührerkopf }} & : & \text { Strömungskraft auf den Rührerkopf } \\ F_{\text {Scher }} & : & \text { Scherkräfte aus Strömung } \\ F_{t} & : & \text { Fluidkraft in tangentialer Richtung } \\ F_{\text {Trägheit }} & : & \text { Trägheitskraft } \\ F_{x} & : & \text { Axiallast } \\ F_{\text {Unwucht }} & : & \text { Unwuchtkraft } \\ F_{\text {Zentrifugal }} & : & \text { Zentrifugalkraft } \\ g & : & \text { Ortsfaktor } \\ h_{B} & : & \text { Blatthöhe } \\ h_{\text {Rührer }} & : & \text { Höhe Rührerkopf } \\ H_{0} & : & \text { Füllhöhe des Rührbehälters } \\ I & : & \text { Flächenträgheitsmoment } \\ k & : & \text { turbulente kinetische Energie } \\ K & : & \text { Steifigkeitsmatrix im Strukturmodell } \\ K_{O} & : & \text { Biegesteifigkeitsfaktor im numerischen Strukturmodell } \\ K_{l} & : & \text { Axialsteifigkeitsfaktor im numerischen Strukturmodell } \\ l & : & \text { Gesamtlänge des Schwingers } \\ l_{E} & : & \text { Länge Einspannträger } \\ l_{l} & : & \text { Lagerabstand } \\ l_{\text {Welle }} & : & \text { Ersatze der freifliegenden Rührerwelle } \\ m & : & \text { Masse pro Balkenlänge } \\ m_{B} & : & \\ M & : & \\ M_{B} & : & \\ & : & \end{array}$




\begin{tabular}{|c|c|c|}
\hline$m_{\text {fluid }}$ & : & fluiddynamische Zusatzmasse \\
\hline$m_{\text {Rührer }}$ & $:$ & Masse Rührerkopf, Endmasse \\
\hline$m_{\text {Welle }}$ & : & Masse Welle \\
\hline$n$ & $:$ & Drehzahl \\
\hline$n_{\text {krit }}$ & : & kritische Drehzahl \\
\hline$n_{\text {Blatt }}$ & $:$ & Blattdrehzahl \\
\hline$N_{B}$ & : & Anzahl Rührerblätter \\
\hline $\mathrm{Ne}$ & $:$ & Newtonzahl (Leistungkennzahl) \\
\hline$N_{F h}$ & : & dimensionslose hydraulische Kraftzahl \\
\hline$q$ & : & Streckenlast \\
\hline$Q$ & $:$ & Querkraft \\
\hline$p$ & $:$ & Druck \\
\hline$p$ & : & Polzahl \\
\hline$P$ & : & Leistung \\
\hline$P_{k}$ & : & Produktionsrate \\
\hline$P e$ & $:$ & Peclet-Zahl \\
\hline$r$ & $:$ & Radius Rührerkopf \\
\hline$r_{i}$ & $:$ & $\begin{array}{l}\text { Abstand Resultierende der Rührerblattflächenkraft - } \\
\text { Wellenachse }\end{array}$ \\
\hline$R$ & : & Quellterm \\
\hline$R$ & : & Verstärkungswiderstand \\
\hline$R e$ & : & Reynoldszahl \\
\hline$S$ & : & Schwerpunkt \\
\hline$t$ & : & physikalische Zeit \\
\hline$t^{0}$ & : & Einheitsvektor in Richtung der Bahntangente \\
\hline$T S$ & : & Zeitschritt \\
\hline$u$ & : & Geschwindigkeitskomponente in $\mathrm{x}$-Richtung \\
\hline$u_{\tau}$ & $:$ & Wandschubspannungsgeschwindigkeit \\
\hline$U$ & : & Umfang Rührbehälter \\
\hline$U$ & $:$ & Umdrehung \\
\hline$v$ & : & Geschwindigkeitskomponente in y-Richtung \\
\hline$V$ & : & Verstärkungsfaktor \\
\hline$V_{D}$ & : & Messvolumen Interferenzmuster \\
\hline$w$ & $:$ & Durchbiegung Biegebalken \\
\hline$w$ & : & Geschwindigkeitskomponente in z-Richtung \\
\hline
\end{tabular}




$\begin{array}{lll}w_{E} & : & \text { Durchbiegung der Einspannung } \\ w_{\text {rel }} & : & \text { relative Anströmgeschwindigkeit } \\ W & : & \text { Wellendurchstoßpunkt } \\ x & : & \text { 1. kartesische Koordinate } \\ X & : & \text { Lagerkraft } \\ y & : & 2 . \text { kartesische Koordinate } \\ y^{+} & : & \text {Modellgröße } \\ y_{\text {Hebel }} & : & \text { Hebelarm } \\ y_{W} & : & \text { Auslenkung des Wellendurchstoßpunkts in y-Richtung } \\ y_{S} & : & \text { Auslenkung des Schwerpunkts in y-Richtung } \\ \hat{Y}_{\text {Luft }} & : & \text { maximale Amplitude bei } \mathrm{n}_{\text {krit }} \text { im Leerlauf } \\ \hat{Y}_{\text {Fluid }} & : & \text { maximale Amplitude bei } \mathrm{n}_{\text {krit }} \text { im Fluid } \\ z & : & \text { Kugelanzahl } \\ z & : & \text { 3. kartesische Koordinate } \\ z_{W} & : & \text { Auslenkung des Wellendurchstoßpunkts in z-Richtung } \\ z_{S} & : & \text { Auslenkung des Schwerpunkts in z-Richtung }\end{array}$

\section{Griechische Symbole}

$\begin{array}{lll}\alpha & : & \text { Gewichtungsfaktor } \\ \alpha & : & \text { Stabilitätsparameter im Newmark-Verfahren } \\ \beta & : & \text { Eigenwert des Kontinuumschwingers } \\ \gamma & : & \text { Winkel } \\ \delta & : & \text { Abklingkoeffizient } \\ \delta_{i j} & : & \text { Kronecker-Symbol } \\ \varepsilon_{c} & : & \text { Dämpfungskennwert } \\ \varepsilon & : & \text { Exzentrizität } \\ \varepsilon & : & \text { Dissipationsrate der turbulenten kinetischen Energie } \\ \phi & : & \text { allgemeine Strömungsgröße } \\ \varphi & : & \text { Umfangswinkel } \\ \Gamma & : & \text { Diffusionskoeffizient } \\ \eta & : & \text { dynamische Viskosität }\end{array}$




$\begin{array}{lll}\Lambda & : & \text { Logarithmisches Dekrement } \\ \lambda & : & \text { Konstante } \\ \mu & : & \text { längenbezogene Last } \\ \mu & : & \text { Strömungsreibung } \\ \mu_{t} & : & \text { Wirbelviskosität } \\ v & : & \text { Dämpfungsexponent } \\ \theta & : & \text { Phasenverschiebung } \\ v & : & \text { kinematische Viskosität } \\ \rho & : & \text { Dichte } \\ \sigma_{F} & : & \text { Streckgrenze } \\ \omega & : & \text { Winkelgeschwindigkeit } \\ \tau & : & \text { Wandscherspannung } \\ \tau_{i j} & : & \text { Spannungstensor } \\ \varsigma & : & \text { Strukturdämpfungskonstante } \\ \varsigma_{B} & : & \text { Strukturdämpfungskonstante pro Balkenlänge } \\ \psi & : & \text { Widerstandsbeiwert } \\ \Omega & : & \text { Winkelgeschwindigkeit }\end{array}$

\section{Fußzeiger}

$\begin{array}{lll}1,2,3 & : & \text { Zeiger für Bereiche der Biegelinie } \\ I & : & \text { Numerischer Zähler in } x \text {-Richtung } \\ i & : & \text { Indizierung kartesischer Tensoren oder Zählvariable } \\ J & : & \text { Numerischer Zähler in } y \text {-Richtung } \\ j & : & \text { Indizierung kartesischer Tensoren oder Zählvariable } \\ E & : & \text { Einspannsystem } \\ K & : & \text { Kugelboden } \\ S & : & \text { Scheibe } \\ T & : & \text { Torsion }\end{array}$




\section{Einleitung}

\subsection{Bedeutung von Rührwerken in der Industrie}

Rühren und Mischen sind fundamentale Prozesse der Verfahrenstechnik und stellen für sehr viele Produkte einen Teilschritt bzw. sogar den wichtigsten Schritt im verfahrenstechnischen Herstellungsprozess dar. Rührwerke werden vor allem in der verfahrenstechnischen und chemischen Industrie in vielfältiger Weise zur Realisierung der Grundoperation „Mischen“ eingesetzt. Dieser Anwendungsbereich umfasst die Teilgebiete [53], [57], [108], [121]:

- Homogenisieren (Ausgleichen von Konzentrations- und Temperaturunterschieden)

- Aufwirbeln bzw. Suspendieren (gleichmäßiges Verteilen eines Feststoffes im Fluid)

- Dispergieren flüssig-flüssig (Feinverteilung einer Flüssigkeit in einer anderen Flüssigkeit)

- Dispergieren gasförmig-flüssig (Zerteilen eines Gases in einer Flüssigkeit)

- Intensivieren des Wärmeaustauschs zwischen Flüssigkeit und Wärmeaustauschfläche

Hierbei muss unterschiedlichsten prozesstechnischen Anforderungen Rechnung getragen werden: Viskositäten bis zu 1000Pas [41], Stoffübergangsprobleme in Mehrphasengemischen und die Wärmeabfuhr bis $\mathrm{zu} 1 \mathrm{MW} / \mathrm{m}^{3}$. Oftmals müssen bei komplexen Rühraufgaben mehrere der oben aufgeführten Rühraufgaben gleichzeitig bewältigt werden. In diesen Fällen ist die dominierende Rühraufgabe besonders zu berücksichtigen.

Von den rund 130 Rührerformen sind die bedeutendsten in der DIN 28131 beschrieben [75]. Der Rührkesselinhalt variiert dabei von einem Liter im Labormaßstab bis zu mehr als $100 m^{3}[18]$.

Zur Auswahl von Rührwerken gibt es kein einheitliches Konzept. Die einzelnen Branchen setzen die Erfüllung höchst unterschiedlicher betriebswirtschaftlicher und technischer Randbedingungen voraus. 


\subsection{Rührwerksschwingungen}

In allen Bereichen des Maschinenbaus und der Anlagentechnik stehen heute Fragen der Betriebsfestigkeit und der Lebensdauer von Maschinen, Anlagen und Bauteilen im Vordergrund der Betrachtungen. Durch Vermeidung von kritischen Zuständen in Form von Resonanzen und Amplitudenvergrößerungen bei dynamischen Vorgängen können diese Systeme stärker belastet werden. Dazu ist die genaue Kenntnis dieser Größen von entscheidender Bedeutung. Insbesondere im Konstruktionsstadium ist es vielfach möglich, aufgrund der Kenntnis des dynamischen Verhaltens eines Apparats in Verbindung mit gezielt vorgenommenen konstruktiven Veränderungen die Beanspruchung und Lebensdauer eines derartigen Systems erheblich zu vergrößern.

An Rührwerken können erzwungene Schwingungen aus Biege- und Torsionsbeanspruchung resultieren. Ursache für diese Schwingungen sind pulsierende Momente. Diese periodischen Anregungen erfolgen gewöhnlich im Takt des Wellenumlaufs, wie z.B. bei der

- Unwuchtanregung als häufigster Anregungsursache, oder mit vielfacher Wellenumlauffrequenz, wie z.B. bei der

- fluidinduzierten Anregung.

Trotz der weitverbreiteten Anwendungsgebiete werden Rührwerke auch heutzutage noch weitgehend empirisch, mit aufwendigen experimentellen Verfahren oder ungenauen analytischen Lösungen sicherheitstechnisch ausgelegt.

Fehlende Berechnungsgrundlagen und Modelle sind oftmals die Ursache für Falschauslegungen und damit verbundene Schadensfälle; so auch bei der Entwicklung von freifliegenden, schnelllaufenden Rührwerken in Fluiden, für deren korrekte Auslegung die Eigenfrequenz bzw. die kritische Drehzahl $n_{k r i t}$ eine wichtige Größe darstellt.

Stimmen Betriebsdrehzahl $n$ und kritische Drehzahl $n_{\text {krit }}$ überein, kommt es zu Resonanzschwingungen mit maximalen Biegemomenten an der Rührerwelle. Bei Resonanz unterliegen die Rührerwellen als ungedämpfte oder schwach gedämpfte Schwingungssysteme unendlich großen bzw. im resonanznahen Bereich sehr erheblichen Durchbiegungen und Verdrehwinkeln, deren Folge Wellenbrüche sein können.

Bei der Bestimmung der kritischen Drehzahl $n_{k r i t}$ von Rührwerken wurde in der Vergangenheit in der Industrie zum Teil von falschen Berechnungsgrundlagen 
ausgegangen, die im schlimmsten Fall zu Schäden am Rührwerk geführt haben [12] [103], [104]. Bei Schäden an Rührerwelle oder Rührorgan ist in der Regel mit nicht unerheblichen Reparatur- und Folgekosten zu rechnen [53]. Es gilt daher, den Resonanzbereich zu bestimmen, und als Dauerbetrieb unbedingt zu vermeiden [115].

Das komplexe Wechselspiel zwischen der Mechanik des Rührers und den auf den Rührer einwirkenden fluiddynamischen Effekten, lassen eine geschlossene analytische Lösung des dynamischen Verhaltens des Rührwerks nicht zu.

Des Weiteren kann die Art der Rührwerksbefestigung am Behälterdach in einer analytischen Lösung nicht berücksichtigt werden. Hier wird oftmals von einer starren Einspannung ausgegangen, die in der Realität selten zutrifft.

Gerade in der heutigen Zeit der Prozessoptimierung werden auch Rührwerke immer mehr an ihre sicherheitstechnischen Belastungsgrenzen gefahren, welche mit den bisherigen Auslegungsmethoden oftmals nur ungenau oder nur mit erheblichem experimentellem Aufwand bestimmt werden können. 


\subsubsection{Betrieb im Leerlauf (Luft)}

Für den Fall der masselosen Rührerwelle wird das Rührwerk im Leerlauf durch folgende Kräfte belastet (siehe Abbildung 1.1).

- $\quad F_{\text {Zentrifugal }}=\left(F_{\text {Trägheit }}+F_{\text {Unwucht }}\right)$

- $F_{\text {elast }}$

- $F_{G}$

- $\quad F_{\text {Dämpfung }}$

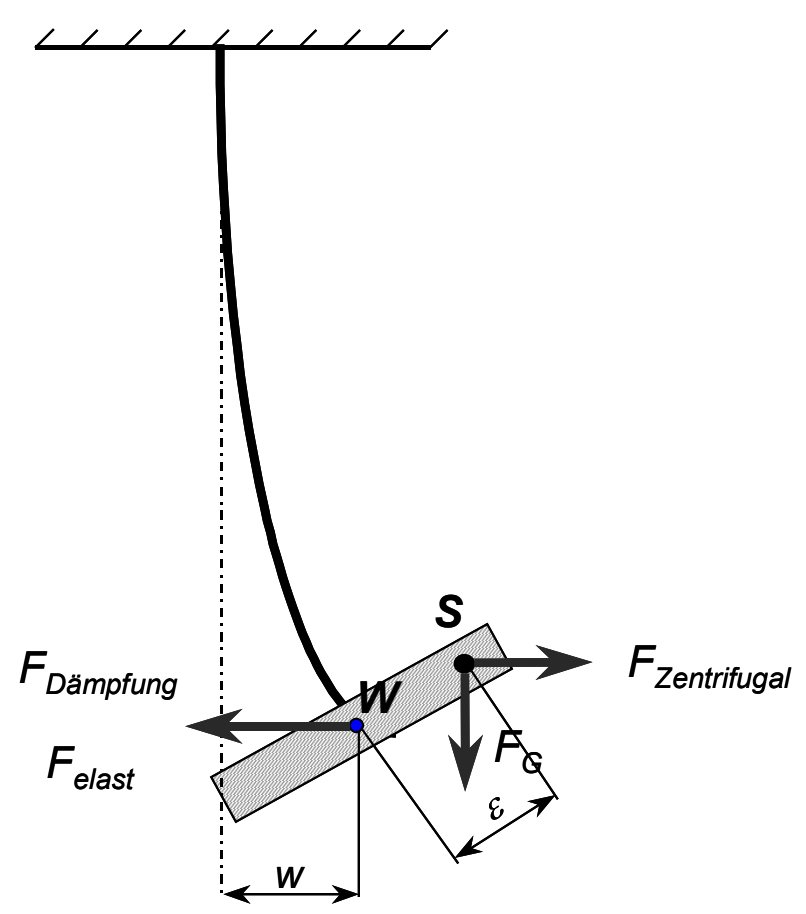

Abbildung 1.1: Kräfte auf das Rührwerk bei Betrieb im Leerlauf

Für den Fall des dämpfungsfreien Betriebs liegen der Wellenmittelpunkt $W$ im Ausgangszustand, der Wellenmittelpunkt $W$ im ausgelenkten Zustand und der Schwerpunkt $S$ auf einer Geraden und somit die Anregungskraft und die Auslenkung in Phase ohne Winkelverschiebung zueinander. Dämpfungsfrei bedeutet, dass sowohl innere Materialdämpfung als auch äußere Dämpfungseinflüsse, z.B. vom Fluid, vernachlässigt werden. Unter diesen Voraussetzungen liegt ein quasi-stationärer Verformungszustand vor und es kann im mitdrehenden Relativkoordinatensystem für den Leerlauf (Luft) folgendes statisches Kräftegleichgewicht angesetzt werden [37]:

$F_{\text {Zentrifugal }}=F_{\text {elast }}$

Die am Schwerpunkt angreifende Gesamtzentrifugalkraft hat die Größe:

$F_{\text {Zentrifugal }}=(\varepsilon+w) \cdot m_{\text {Rührer }} \cdot n^{2}$ 
Wird die Drehzahl gesteigert, so wächst auch die Zentrifugalkraft zunächst quadratisch mit der Drehzahl an. Ab einer gewissen Drehzahl sind die elastischen Verformungen $w$ des Rührwerks so groß, dass sie in Gleichung (1.2) einen erheblichen Einfluss auf die Zentrifugalkraft haben.

Die elastische Rückstellkraft der Welle wird mit $w \cdot c$ beschrieben, wobei $c$ die Biegesteifigkeit der Welle darstellt.

Die elastische Rückstellkraft der Welle hält der Zentrifugalkraft das Gleichgewicht, so dass sich aus

$c \cdot w=(w+\varepsilon) \cdot m_{\text {Rührer }} \cdot n^{2}$

die Wellenverformung

$$
w=\varepsilon \cdot \frac{(n / \omega)^{2}}{1-(n / \omega)^{2}}
$$

oder anders geschrieben

$$
w=\frac{\varepsilon}{(\omega / n)^{2}-1}
$$

ergibt. Für $\sqrt{c / m_{\text {Rührer }}}$ wird abkürzend $\omega$ geschrieben. Dieser Wert stellt die Eigenkreisfrequenz oder kritische Drehzahl eines Schwingers dar.

Für $\omega / n=1$ ist diese Funktion nicht definiert, d.h. sie geht mit steigender Drehzahl gegen Unendlich und nähert sich bei weiterer Drehzahlsteigerung dem Wert $\varepsilon$ an (siehe Abbildung 1.2).

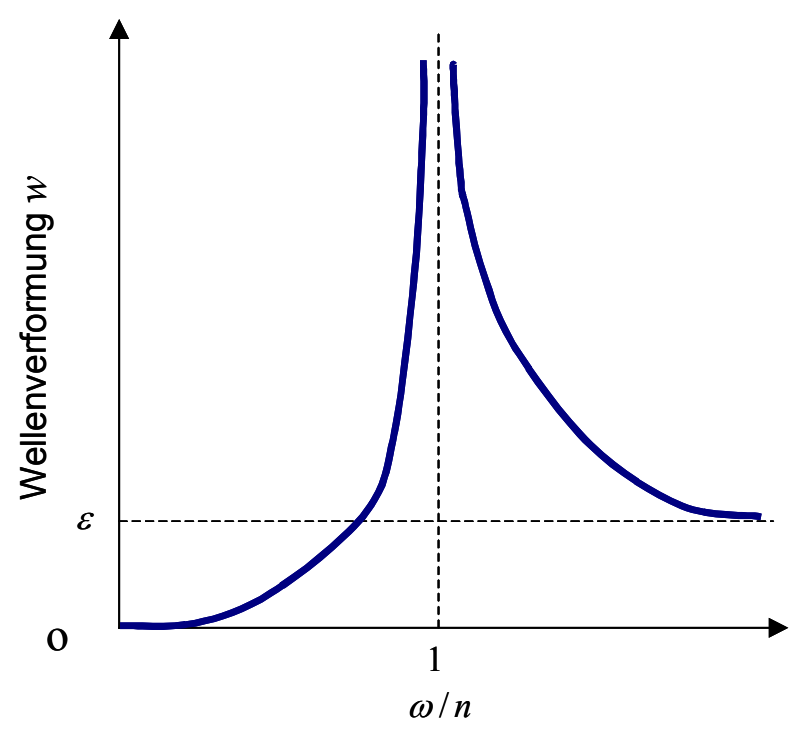

Abbildung 1.2: Wellenauslenkung $w$ beim elastischen Rotor 
Es bleibt jedoch festzuhalten, dass die Reduktion des Kräftegleichgewichts auf Gleichung (1.1) nur für den idealisierten, dämpfungsfreien Fall gilt. Für den dämpfungsbehafteten Fall muss das dynamische Kräftegleichgewicht (siehe Kapitel 2) aufgestellt werden.

\subsubsection{Betrieb im Fluid}

Bei Betrieb im Rührmedium treten zusätzlich folgende Fluidkräfte auf das Rührorgan auf, die in Kapitel 2.5 noch ausführlich behandelt werden:

- fluiddynamische Zusatzmasse

- fluiddynamische Dämpfungskräfte

- $\quad$ fluiddynamische Querkräfte

- Turbulenzkräfte

Diese Fluidkräfte bewirken eine Veränderung der Schwingungsamplitude des Rührwerks und eine Verschiebung der kritischen Drehzahl zu kleineren Werten.

Des Weiteren können sie im unterkritischen Drehzahlbereich als sich periodisch wiederholende Schwingungsanregende wirken (siehe Kapitel 2.5.4).

Bei turbulenter Strömung im Rührbehälter treten auf das Rührerblatt überwiegend Druckkräfte auf, welche sich mit

$$
F \sim \rho \cdot n^{2} \cdot d_{\text {Rührer }}^{2}
$$

berechnen lassen.

Als Proportionalitätskonstante wird der Kraftbeiwert $C_{F}$ mit

$$
C_{F}=\frac{F}{\rho \cdot n^{2} \cdot d_{\text {Rührer }}^{4}}
$$

eingeführt, welcher die hydraulischen Belastungen der Rührerwelle dimensionslos beschreibt.

Bei genauer Betrachtung der Strömungskräfte lassen sich diese am Rührerblatt in Axial- $F_{a x}$ bzw. Tangentialanteile $F_{t}$ aufteilen. 


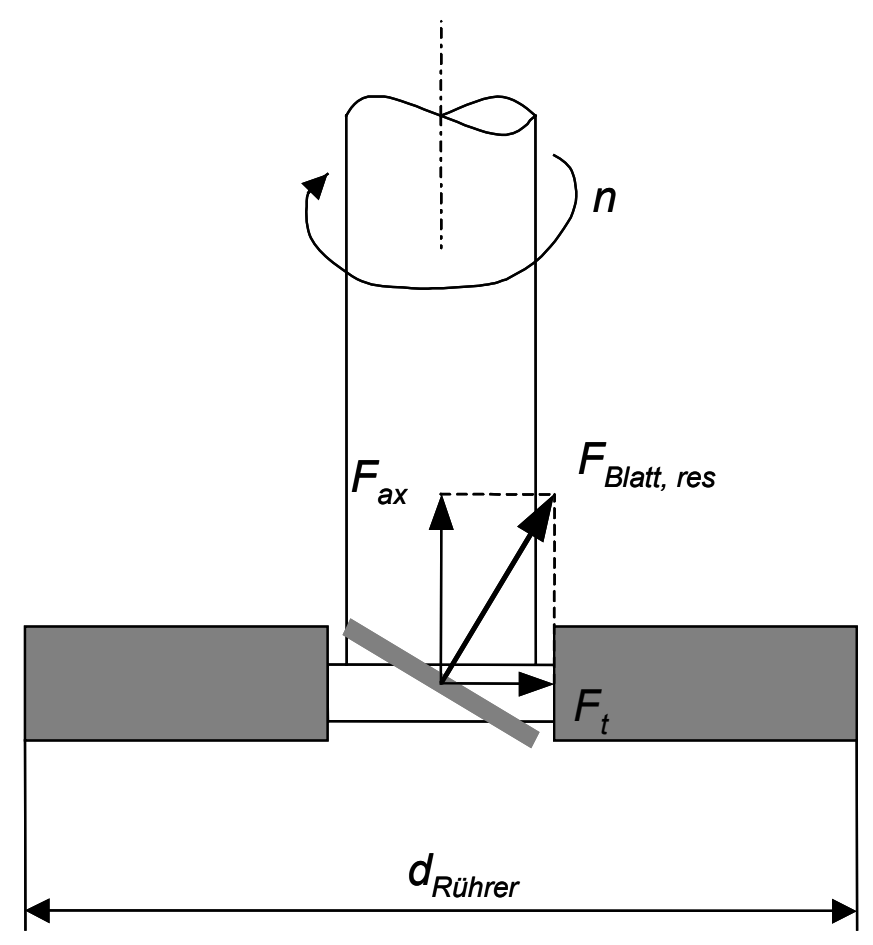

Abbildung 1.3: Axial- und Tangentialkräfte am Rührerblatt.

Die jeweiligen Kraftbeiwerte in Axial-, Radial- und Tangentialrichtung können mit Gleichung (1.7) durch Einsetzen der entsprechenden Kräfte bestimmt werden. Der Tangential-Kraftbeiwert $C_{t}$ ist hierbei direkt proportional zur Leistungskennzahl $\mathrm{Ne}$.

Im laminaren Strömungsbereich überwiegt aufgrund der erhöhten Reibung zwischen Rührorgan und Fluid die Tangentialkraft, im turbulenten Strömungsbereich nimmt die Axialkraft größere Werte an [108]. 


\subsection{Bisherige Lösungsansätze zur Bestimmung der kritischen Drehzahl}

\subsubsection{Analytische Ansätze für den Betrieb im Leerlauf}

Nachfolgend werden drei einfache analytische Lösungen für die Bestimmung der ersten Eigenfrequenz einer einseitig starr eingespannten Welle in Luft vorgestellt. Es wird eine glatte Welle mit konstantem Querschnitt vorausgesetzt. Je nach ErsatzModell treten eine konstante Massenverteilung über der Welle $m_{\text {Welle }}$ und/oder eine Endmasse $m_{\text {Rührer }}$ auf.

Gleichung (1.8) wird in der Literatur [6], [120] allgemein als Formel zur Berechnung der kritischen Drehfrequenz einer Welle mit fliegendem Wellenende angegeben. Das Eigengewicht der Rührerwelle wird vernachlässigt, es wird nur eine Endmasse $m_{\text {Rührer }}$ berücksichtigt.

In diesem Fall handelt es sich bei dem betrachteten System um einen Feder-MasseSchwinger, und die erste Eigenfrequenz wird nach der Gleichung

$$
f_{1}=\frac{1}{2 \pi} \sqrt{\frac{c}{m_{\text {Rührer }}}}
$$

berechnet. Dabei muss die Steifigkeit $c$ aus einer linearelastischen Analyse eines einseitig fest eingespannten Balkens mit Kreisquerschnitt berechnet werden. Es ergibt sich [95]:

$c=\frac{3 E I}{l_{\text {Welle }}^{3}}$

Die erste Eigenfrequenz berechnet sich damit nach der Gleichung:

$$
f_{1}=\frac{\beta_{1}^{2}}{2 \pi} \sqrt{\frac{E I}{m_{\text {Rührer }} \cdot l_{\text {Welle }}^{3}}}
$$

mit $\beta_{1}^{2}=\sqrt{3}$ als erstem Eigenwert.

Gleichung (1.12) beschreibt die kritische Drehzahl mit konstanter, längenbezogener Last $\mu=m_{\text {Welle }} / l_{\text {Welle }}$ hinsichtlich des Balkengewichts $m_{\text {Welle }}$ ohne Endmasse $m_{\text {Rührer }}$. In diesem Fall muss die Welle als kontinuierlicher Schwinger angesehen werden. Für die Eigenwerte des kontinuierlichen, einseitig eingespannten Balkenschwingers ergibt sich die Eigenwertgleichung [80]:

$1+\cos \beta \cdot \cosh \beta=0$ 
Der erste Eigenwert ist $\beta_{1}=1,8751$. Mit diesem Eigenwert kann nun nach Gleichung (1.11) die Eigenfrequenz des Balkenschwingers berechnet werden:

$f_{1}=\frac{3,52}{2 \pi} \sqrt{\frac{E I}{\mu l_{\text {Welle }}^{4}}}$

Die Bestimmung der kritischen Drehzahl eines realen Rührwerks erfordert die Berücksichtigung der Wellenmasse $m_{\text {Welle }}$ und der Endmasse $m_{\text {Rührer }}$. Nach Kantorowitsch [48] sind der Einzelmasse des Rührorgans ca. 24\% der gesamten Wellenmasse aufzuschlagen. Entsprechend ist Gleichung (1.10) zu modifizieren, so dass gilt:

$$
f_{1}=\frac{1,732}{2 \pi} \sqrt{\frac{E I}{m_{\text {Rührer }} \cdot l_{\text {Welle }}^{3}+0,236 \cdot \mu l_{\text {Welle }}^{4}}}
$$

Es sei nochmals darauf hingewiesen, dass die Gleichungen (1.10) - (1.13) nur in Luft (Leerlauf) gelten, und eine starre Anschlusssteifigkeit des Rührwerks vorausgesetzt wird.

Typische Sicherheitsabstände für Rührwerke von ihrer ersten kritischen Drehzahl sind laut Melzer [66] $0,71 \cdot n_{k r i t}-1,23 \cdot n_{k r i t}$. Nach Todtenhaupt [108] sollte der Sicherheitsabstand von der kritischen Drehzahl im Allgemeinen $\pm 20 \%$ betragen. Als Bezugsgröße wird die kritische Drehzahl in Luft (Leerlauf) verwendet. Eine im Betrieb veränderte kritische Drehzahl im Rührmedium kann daher zu einer Fehleinschätzung des kritischen Bereichs führen [54].

Es konnte in Arbeiten unterschiedlicher Autoren nachgewiesen werden, dass - im Vergleich zum Betrieb im Leerlauf - die kritische Drehzahl im Rührgut verringert wird. Pauschalaussagen über die Größe der Verringerung sind nicht möglich, da sowohl die Rührerkopfgeometrie als auch das Rührmedium einen entscheidenden Einfluss haben [51], [52].

\subsubsection{Analytische Erweiterungen für den Betrieb im Fluid}

Im Rührbehälter besteht eine Wechselwirkung zwischen dem Rührorgan als Ursache des Strömungsfeldes und der rückwirkenden Beeinflussung der Rührwerksdynamik durch strömungsbedingte Kräfte. Die verschiedenen Einflüsse des Fluids auf die kritische Drehzahl und die dazugehörige Auslenkungsamplitude des Rührwerks können analytisch nur teilweise, und dann nur mit erheblichem rechnerischen Aufwand 
bestimmt werden. So wird in den meisten analytischen Ansätzen nur eine fluid- oder hydrodynamische Zusatzmasse berechnet, welche der Rührwerksmasse aufgeschlagen wird. Eine Berücksichtigung aller auftretenden transienten Fluideinflüsse auf das Rührwerk ist auf analytischem Wege nicht zu realisieren.

In den folgenden analytischen Ansätzen werden die in der Literatur gefundenen analytischen Ansätze für die Bestimmung der kritischen Drehzahl in Fluiden vorgestellt.

Kantorowitsch [48] berechnet die hydrodynamische Zusatzmassse $m_{\text {hyd }}$ in grober Näherung über das Volumen, welches ein Rührerblatt in der Umdrehung einnimmt. Somit ergibt sich für Blatt- und Schaufelrührer:

$$
m_{\text {hyd }}=\rho \int_{d_{i} / 2}^{d_{\text {Rührer }} / 2} h_{\text {Rührer }} 2 \pi r d r=\frac{h_{\text {Rührer }} d_{\text {Rührer }}^{2}}{4}\left[1-\left(\frac{d_{i}}{d_{\text {Rührer }}}\right)\right]
$$

wobei $d_{i}$ den doppelten inneren Abstand der Blattwurzelpunkte von der Wellenachse darstellt.

Liepe [61] bestimmt die hydrodynamische Zusatzmasse aus einem auf jeder Schaufelseite sitzenden, dachförmigem Volumen, das allseitig um $60^{\circ}$ abgeschrägt ist. Für einen Blattrührer gilt somit:

$$
m_{\text {hyd }}=0,5 \cdot \sqrt{3} \cdot \rho \cdot d_{\text {Rührer }}^{3} \cdot\left(1-0,33 \cdot \frac{h_{\text {Rührer }}}{d_{\text {Rührer }}}\right)
$$

Für einen Scheibenrührer vereinfacht sich die Gleichung zu:

$$
m_{\text {hyd }}=0,38 \cdot \rho \cdot d_{\text {Ruhrer }}^{3}
$$

Die hydrodynamische Zusatzmasse wird bei der analytischen Berechnung der Eigenfrequenz zur Masse des Rührorgans hinzuaddiert.

Somit lautet Gleichung (1.13) nun:

$$
f_{1}=\frac{1,732}{2 \pi} \sqrt{\frac{E I}{\left(m_{\text {Rinhrer }}+m_{\text {hyd }}\right) \cdot l_{\text {Welle }}^{3}+0,236 \cdot \mu l_{\text {Welle }}^{4}}}
$$

Burger [22] beschreibt, dass die übrigen drei fluiddynamischen Einflussparameter Dämpfung, Quertrieb und Turbulenz auf das Biegeschwingungsverhalten von Rührwerken stark von der Rührergeometrie und vom Rührgut abhängen. Ihre Bestimmung erfordert einen erheblichen experimentellen und mathematischen Aufwand. 
Für die Eigenfrequenzverschiebung infolge der äußeren Dämpfung durch das Fluid gibt Burger [22] mögliche Bereiche bzw. praktische Werte an, wie etwa eine Absenkung von $n_{k r i t}$ um $11 \%-23 \%$.

Bei Milchenko [69] beträgt der Absenkungsbereich beim Propellerrührer je nach Viskosität des Rührguts zwischen $4 \%$ und $23 \%$.

Knoch [54] bestimmt die kritische Drehzahl im Fluid ausgehend von zwei dimensionslosen Kennzahlen $D K_{3}$ und $D K_{4, R u ̈}$ :

$$
n_{\text {krit }, \text { Fluid }}=\frac{n_{\text {krit Luft }} \cdot\left(C_{n_{\text {krit }} 1} \cdot \ln \left(\frac{\eta}{n_{\text {krit }, \text { Luft }} \cdot d_{\text {Rührer }}^{2} \cdot \rho} \cdot \frac{A_{B}}{A_{R}}\right)+C_{n_{\text {krit }} 2}\right)}{\left(\frac{m_{\text {Rührer }} \cdot n_{k r i t, \text { Luft }}}{\eta \cdot h_{B}}\right)^{0,03} \cdot\left(\frac{D}{h_{B}}\right)^{0,04} \cdot N_{B}^{0,015}}
$$

Weiterhin berechnet Knoch die radialen Kräfte im Fluid bei kritischer Drehzahl zu:

$$
F_{r, \text { Fluid }}=\frac{F_{r, \text { Luft }} \cdot\left(C_{F_{R} 1} \cdot \ln \left(\frac{\eta}{n_{\text {krit Luft }} \cdot \hat{Y}_{\text {Luft }}^{2} \cdot \rho} \cdot \frac{A_{R}}{\hat{Y}_{\text {Luft }}}\right)+C_{F_{R} 2}\right)}{\left(\frac{m_{\text {Rührer }} \cdot n_{\text {krit }, \text { Luft }}}{\eta \cdot d_{\text {Rührer }}}\right)^{0,01} \cdot\left(\frac{\hat{Y}_{\text {Luft }}}{d_{\text {Rührer }}+h_{B}}\right)^{0,3} \cdot N_{B}^{1,38} \cdot\left(\frac{A_{B}}{A_{R}}\right)^{0,7}}
$$

Die resultierende Wellenauslenkung folgt mit:

$$
\hat{Y}_{\text {Fluid }}=\frac{F_{r, \text { Fluid }} \cdot l_{2}^{3}}{K_{1} \cdot 3 \cdot E \cdot I y} \cdot\left(\frac{l_{1}}{l_{\text {Welle }}}+1\right)
$$

Die Konstanten $C_{n_{k r i t} 1}, C_{n_{k r i t} 2}, C_{F_{R} 1}$ und $C_{F_{R} 2}$ sind von der Rührerkopfgeometrie abhängig und müssen über Experimente bestimmt werden. Laut Knoch fehlen Untersuchungen, in denen die Stoffparameter Dichte und Viskosität des Rührgutes systematisch variiert werden.

Bei den meisten in der Literatur dargestellten Untersuchungen zur kritischen Drehzahl im Fluid wurden nur wenige einfache Standardrührwerke im Rührmedium Wasser eingesetzt. Die Ergebnisse lassen sich somit kaum auf komplexere Rührorgantypen in anderen Rührmedien übertragen. 


\subsubsection{Numerische Ansätze}

Die am häufigsten verwendeten numerischen Lösungen zur Berechnung von Eigenfrequenzen sind das

- Näherungsverfahren von RITZ (Variationsmethoden)

- Verfahren von GALERKIN (gewichtete Residuen)

und das

- Verfahren von RAYLEIGH.

Diese Verfahren erfordern den Einsatz von Computern und werden auch in FiniteElement-Programmen verwendet [65].

Eigene Untersuchungen [9] mit FEM-Analysen ergaben, dass durch Modalanalysen die Eigenfrequenzen von Rührwerken in Luft (Leerlauf) sehr genau bestimmt werden können. Der Einfluss des Rührgutes konnte aber auch hier, wie in den oben aufgeführten analytischen Ansätzen, lediglich über die fluiddynamische Zusatzmasse geltend gemacht werden [64]. Eine gleichzeitige numerische Berechnung der turbulenten Strömungskräfte ist zum gegenwärtigen Zeitpunkt noch in keinem FEMCode implementiert. 


\subsection{Schadensfälle}

Fehler bei der sicherheitstechnischen Auslegung von Rührwerken können im schlimmsten Fall zu Schäden an Rührwerk und Rührbehälter führen [9], [103], [104], [105]. Wellenbrüche sind die schlimmste Folge einer Resonanzkatastrophe $\left(n \approx n_{\text {krit }}\right)$, die aus einer Falschauslegung eines Rührwerks resultiert. Häufiger kommt es jedoch zu Lagerschäden oder plastischen Wellenverformungen, die allerdings ebenfalls erhebliche Reparatur und vor allem Folgekosten aufgrund von Stillstandszeiten zur Folge haben.

Exemplarisch soll in den folgenden Abbildungen ein Schadensfall dokumentiert werden, der sich bei den zu dieser Arbeit durchgeführten experimentellen Untersuchungen am Lehrstuhl für Apparate- und Anlagenbau zugetragen hat:

Bei dem Versuch, die kritische Drehzahl $n_{\text {krit }}$ des Rührwerks zu überschreiten, um in den Bereich der Selbstzentrierung des Rührwerks zu kommen, wurden im Resonanzbereich die Schwingungsamplituden so groß, dass der Rührerkopf letztendlich mit den Strombrechern kollidierte (siehe Abbildung 1.3). Es lässt sich somit rückschließen, dass das Wellenende eine Auslenkung von ca. 0,35m erfuhr.

Aufgrund der massiven Bauweise der Strombrecher und dem sofortigen Abschalten der Anlage wurden die Strombrecher nur oberflächlich beschädigt.

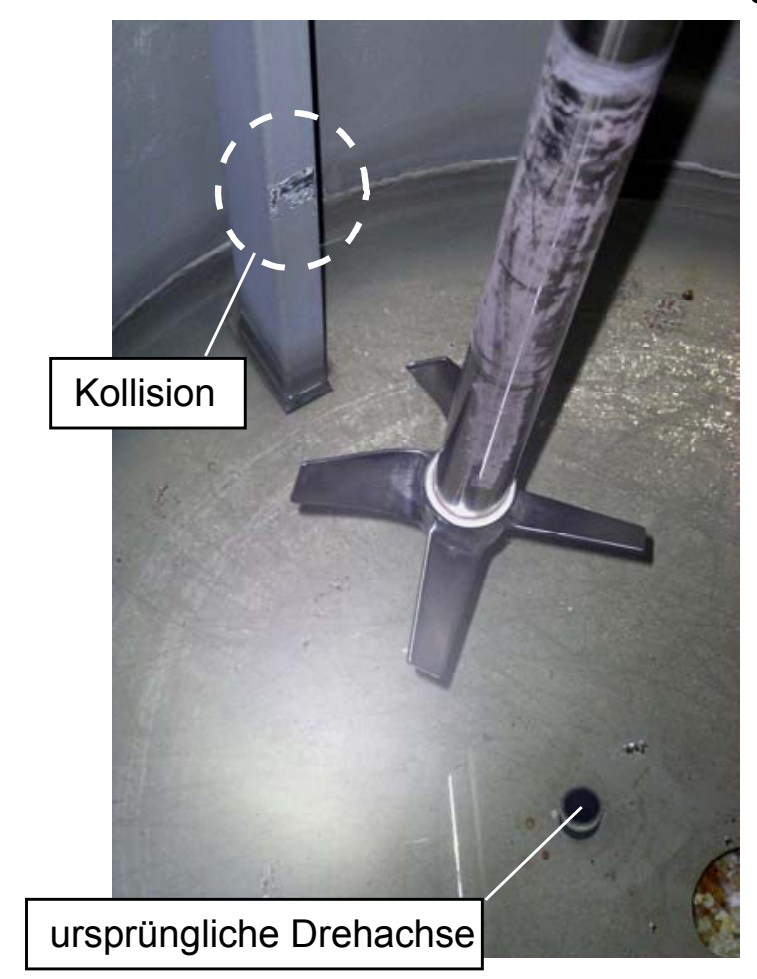

Abbildung 1.3: Kollision zwischen Rührerkopf und Strombrecher im Bereich der kritischen Drehzahl 
Die massive Vollwelle aus Edelstahl $\left(l_{\text {Welle }}=1,34 \mathrm{~m}\right)$ mit einen Durchmesser $d_{\text {Welle }}$ von $0,04 m$ verformte sich durch die Fliehkraft plastisch um $0,12 m$ am Wellenende (siehe Abbildung 1.4).

Es wurde nachträglich analytisch berechnet, dass Fließen $\left(\sigma_{F}=200 \mathrm{~N} / \mathrm{mm}^{2}\right) \mathrm{der}$ äußersten Wellenfaser ab einer Belastung von mindestens $95 \mathrm{~kg}$ eintritt [106]. Da bei diesem Schadensfall mehrere Fasern plastisch verformt wurden, kann davon ausgegangen werden, dass ein Vielfaches der berechneten Masse im Betrieb auf die Rührerwelle wirkte und somit zu dieser beträchtlichen plastischen Verformung führte.

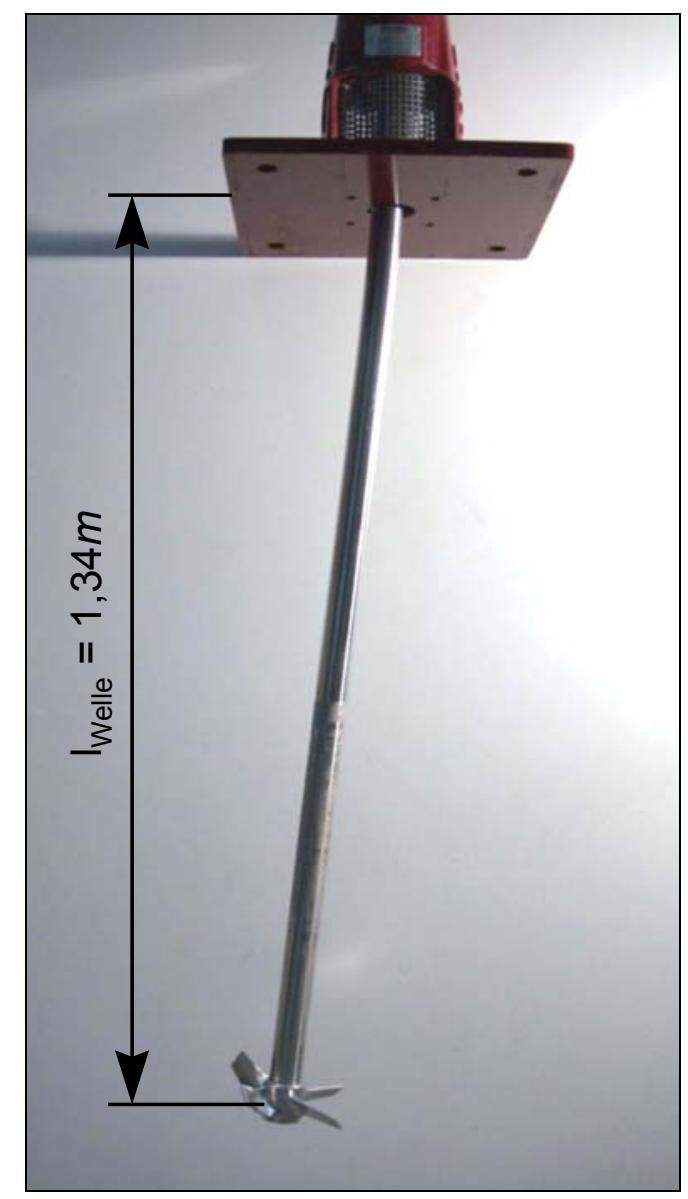

Abbildung 1.4: Plastische Verformung der Vollwelle

Durch die Kollision mit den Strombrechern wurden zwei Blätter des Schrägblattrührers erheblich deformiert, so dass dieser nicht mehr funktionstüchtig war (siehe Abbildung 1.5). 


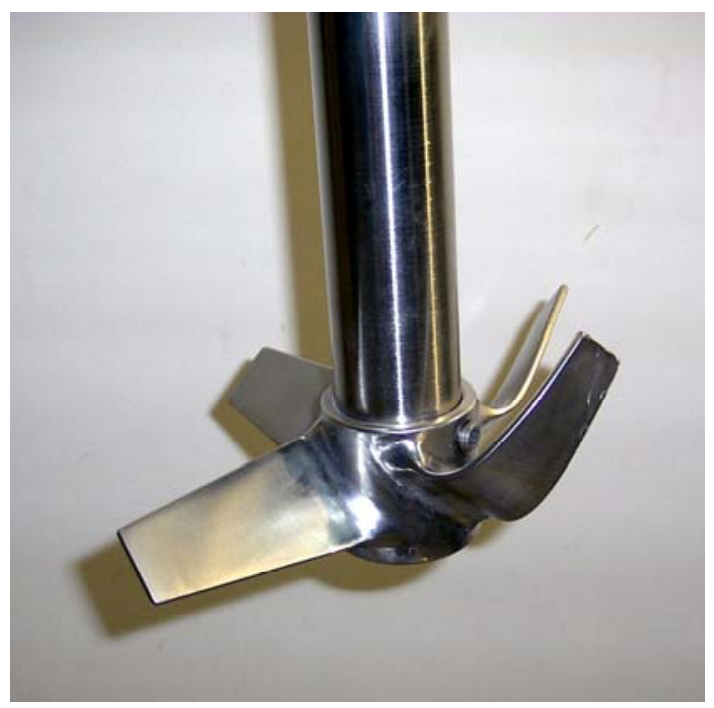

Abbildung 1.5: Deformierter Schrägblattrührer

Ein weiterer Schadensfall [104], [105] an einem Rührwerk mit Leitrohr war in der Tatsache begründet, dass bei der sicherheitstechnischen Auslegung seitens des Rührwerkherstellers sowohl die Fluideinflüssse als auch die nicht als starr zu bezeichnende Einspannsteifigkeit des Rührwerks im Behälterdach vernachlässigt wurden.

In der analytischen Auslegungsrechnung wurde eine kritische Drehzahl von 167U/min in Luft ermittelt, welche sich im Betriebszustand durch die beiden oben genannten Einflüsse jedoch auf $86 \mathrm{U} / \mathrm{min}$ reduzierte. Da das Rührsystem mit 78U/min betrieben wurde, kam es $\mathrm{zu}$ Resonanzschwingungen und somit $\mathrm{zu}$ dem folgenschweren Schadensfall, bei dem das Rührwerk mit dem Leitrohr kollidierte. 


\subsection{Ziel der vorliegenden Arbeit}

Die Voraussetzungen zur Auslegung von Rührwerken nach $n_{\text {krit }}$ mit einfachen analytischen Ansätzen sind selten erfüllt, da die Fluidkräfte auf das Rührwerk und die realen, oftmals weichen Einspannverhältnisse zwischen Rührwerk und Träger nicht hinreichend berücksichtigt werden können. Beide Aspekte haben jedoch einen großen Einfluss auf den Zahlenwert der kritischen Drehzahl $n_{\text {krit }}$ und auf die Auslenkungsamplituden bzw. Biegebeanspruchung des Rührwerks und damit verbundene unzulässige Drehzahlbereiche.

Das Rührmedium kann auf das Rührwerk sowohl als Dämpfungsglied als auch als Anregende fungieren, so dass im Vergleich zum Leerlauf veränderte Auslenkungsamplituden resultieren. Die Stoffeigenschaften des Rührmediums und die Strömungsverhältnisse im Rührbehälter müssen in die Dimensionierung des Rührwerks einfließen. Die Erweiterungen der analytischen Ansätze um fluiddynamische Zusatzmassen gelten oftmals nur für Standardrührwerke und das Rührmedium Wasser, und sind auf Realfälle mit komplexeren Rührorganen nicht zu übertragen. Darüber hinaus sind zur Bestimmung der fluiddynamischen Zusatzmassen häufig kosten- und zeitintensive Vorversuche nötig.

Kommerzielle FEM-Programme sind zum gegenwärtigen Zeitpunkt nicht in der Lage, voll fluid-struktur-gekoppelte Probleme bei praxisrelevanten Reynoldszahlen zu berechnen.

Eine vollständige fluid-struktur-gekoppelte Simulation von FEM-Modell und CFDModell, die über den ständigen Austausch von Größen wie Kräften, Verschiebungen und Geschwindigkeiten zwischen den beiden Programmpaketen laufen müsste, ist sehr rechenzeit- und speicherplatzaufwendig sowie programmiertechnisch zu schwerfällig. In dieser Arbeit wird ein kommerzieller CFD-Code durch ein selbst entwickeltes numerisches Modell für die Rührerdynamik erweitert (Fluid-Struktur-Kopplung). Das simulierte Strömungsfeld wird vollständig mit der Rührwerksstruktur gekoppelt, wodurch sämtliche fluiddynamischen Kräfte auf das Rührwerk berücksichtigt werden. Mögliche weiche Einspannverhältnisse zwischen Rührwerk und Halterung werden über analytische Berechnungskonzepte in das numerische Modell miteinbezogen.

Es können somit sämtliche für die sicherheitstechnische Auslegung von Rührwerken relevanten Einflüsse auf die strukturdynamische Antwort des Rührwerks geltend gemacht werden [21]. 
Durch eine Eingabemaske lassen sich im CFD-Code folgende Randbedingungen des Rührwerks variieren:

- $\quad$ Drehzahl $n$

- Wellenlänge $l_{\text {Welle }}$

- $\quad$-Modul des Rührwerks

- Materialdichte des Rührwerks $\rho$

- Rührmedium

- $\quad$ Einspannsteifigkeit

- unterschiedliche Exzentrizitäten $\varepsilon$

- unterschiedliche Materialdämpfungen $\zeta$

- $\quad$ Rührwerk und Rührbehälter werden im CFD-Code modelliert

Gesamtziel der Arbeit ist eine durch experimentelle Untersuchungen abgesicherte Vorausberechnung der zu vermeidenden Betriebsbereiche verschiedener Rührwerke in unterschiedlichen Fluiden und Einspanngeometrien.

\subsection{Gliederung der Arbeit}

Im folgenden Kapitel 2 wird auf die Theorie des Einmassenschwingers, des kontinuierlichen Schwingers und der Rotordynamik eingegangen. Es werden außerdem die verwendeten strömungsmechanischen Grundgleichungen und der Einfluss des Fluids auf das Biegeschwingungsverhalten von Rührwerken dargestellt. Andere Einflüsse auf die kritische Drehzahl, wie z.B. Einspannsteifigkeiten werden zudem veranschaulicht.

In Kapitel 3 werden die im Rahmen dieser Arbeit entwickelten Berechnungsverfahren beschrieben, die getroffene Auswahl der einzelnen Methoden begründet und die programmtechnische Umsetzung aufgezeigt. Danach erfolgt die Diskussion umfangreicher Testrechnungen an einem Rührwerk im Leerlauf und im Fluid.

Kapitel 4 beschreibt die Experimente, welche zur Absicherung und Beurteilung der Qualitäten des Berechnungsverfahrens herangezogen wurden. Dazu werden in Kapitel 4.1 die Versuchseinrichtungen und die Versuchsdurchführungen zur Bestimmung des Leistungseintrags, der Geschwindigkeitsmessung mit LDA, der Messung der Rührwerksschwingungen und der Druckmessung an den Rührerblättern erläutert. Erste 
experimentelle Ergebnisse, welche als Randbedingung für die Simulation nötig sind, werden vorgestellt.

In Kapitel 5 verifizieren Beispielrechnungen die Anwendbarkeit der Rechenmethode. Hierzu werden die Simulationsergebnisse mit entsprechenden Experimenten verglichen und auftretende Differenzen untersucht.

In Kapitel 6 erfolgt eine abschließende Zusammenfassung und Bewertung der Ergebnisse der vorliegenden Arbeit. 


\section{Theoretischer Grundlagenteil}

\subsection{Mechanik des Einmassenschwingers}

Abbildung 2.1 zeigt das einfachste realitätsnahe Schwingungssystem des FederMasse-Dämpfer-Systems einer Punktmasse unter Fremdanregung.

In diesem System wird die Schwingung einer im Schwerpunkt des Körpers punktförmig angenommenen Einzelmasse durch das Kräftegleichgewicht am freigeschnittenen Körper berechnet.

Nach Schnell, Gross Hauger [96] und Ulbrich [112] lässt sich das dynamische Kräftegleichgewicht eines solchen Systems mit der folgenden Differentialgleichung darstellen:

$m \ddot{x}+\dot{x}+c x=F(t)$

Der erste Term der linken Seite stellt die Trägheitskraft der schwingenden Masse dar, der zweite Ausdruck beschreibt die geschwindigkeitsabhängige Dämpfungskraft mit der Strukturdämpfungskonstante $\varsigma$. Der dritte Summand steht für die Federrückstellkraft mit der Federkonstanten $c$, die proportional zur Auslenkung ist.

Die Summe dieser drei Kräfte steht im Gleichgewicht zur zeitlich veränderlichen äußeren Anregungskraft $F(t)$. Im konkreten Fall des Rührers kann diese Störkraft z.B. eine Unwuchtkraft $F_{\text {Unwucht }}$ oder Fluidkräfte darstellen.

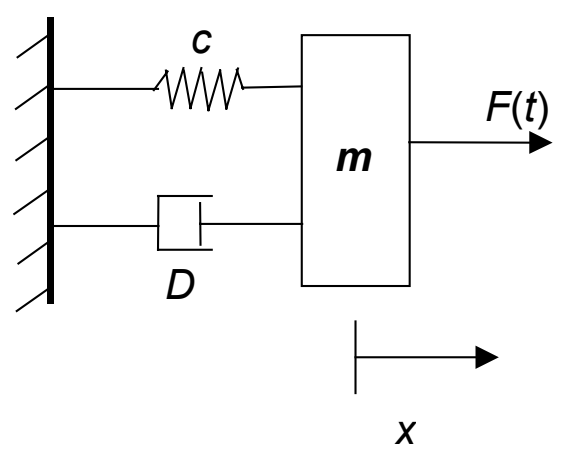

Abbildung 2.1: Modell eines Einmassenschwingers

Die Lösung der Differentialgleichung setzt sich aus der homogenen Lösung $x_{\text {hom }}(F(t)$ wird gleich 0 gesetzt) der Gleichung (2.1)

$m \ddot{x}+s \dot{x}+c x=0$

und der inhomogenen Lösung $x_{\text {inhom }}$ für das Störglied auf der rechten Seite zusammen. Eine Umformung der homogenen Differentialgleichung ergibt: 


$$
\ddot{x}+\frac{\varsigma}{m} \dot{x}+\frac{c}{m} x=0
$$

Durch Einführung der Abkürzungen

$$
2 \delta=\frac{\varsigma}{m} \text { und } \quad \omega^{2}=\frac{c}{m}
$$

erhält man die Gleichung der Form

$$
\ddot{x}+2 \delta \dot{x}+\omega^{2} x=0
$$

Diese lineare Differentialgleichung 2.Ordnung wird mit einem Exponentialansatz $x=A e^{\lambda t} \quad$ mit den Konstanten $A$ und $\lambda$ gelöst [67].

Das Einsetzen des Exponentialansatzes in Gleichung (2.4) liefert

$$
A e^{\lambda t} \lambda^{2}+A e^{\lambda t} 2 \delta \lambda+A e^{\lambda t} \omega^{2}=0
$$

bzw. nach Kürzen

$$
\lambda^{2}+2 \delta \lambda+\omega^{2}=0 \text {. }
$$

Diese quadratische Gleichung hat die beiden Lösungen

$$
\lambda_{1 / 2}=-\delta \pm \sqrt{\delta^{2}-\omega^{2}} .
$$

Durch Einführung des Lehr'schen Dämpfungsmaßes

$$
D \equiv \frac{\delta}{\omega}
$$

lässt sich Gleichung (2.6) auch folgendermaßen schreiben:

$$
\lambda_{1 / 2}=-\delta \pm \omega \sqrt{D^{2}-1}
$$

Im Folgenden lassen sich für die Dämpfung $D$ die drei Fälle $D>1, D=1$ und $D<1$ unterscheiden.

Im Fall $D>1$ liegt starke Dämpfung vor und die Lösungen für $\lambda_{1 / 2}$ werden reell. Der Ausschlag klingt dabei in einer Kriechbewegung mit maximal einem Extremum exponentiell ab. Gleiches gilt für den Fall $D=1$, der auch als, ,aperiodischer Grenzfall“" bezeichnet wird. Hier klingt die Bewegung schneller ab als im Fall $D>1$. Für den Fall $D<1$ wird der Radikand negativ und die beiden Lösungen für $\lambda_{1 / 2}$ komplex.

Gleichung (2.7) liefert dann die Form

$$
\lambda_{1 / 2}=-\delta \pm i \omega \sqrt{1-D^{2}}=1 \pm i \omega_{d}
$$


mit $\omega_{d}=\sqrt{1-D^{2}}$.

Daraus ergibt sich als allgemeine homogene Lösung der Differentialgleichung

$x_{\text {hom }}(t)=A_{1} e^{\lambda_{1} t}+A_{2} e^{\lambda_{2} t}=e^{-\delta t}\left(A_{1} e^{i \omega_{d} t}+A_{2} e^{-i \omega_{d} t}\right)$

mit $e^{ \pm i \omega_{d} t}=\cos \left(\omega_{d} t\right) \pm i \sin \left(\omega_{d} t\right)$

erhält man

$x_{\mathrm{hom}}(t)=e^{-\delta t}\left[\left(A_{1}+A_{2}\right) \cos \left(\omega_{d} t\right)+i\left(A_{1}-A_{2}\right) \sin \left(\omega_{d t}\right)\right]=$

$e^{-\delta t}\left[A \cos \left(\omega_{d} t\right)+i B \sin \left(\omega_{d} t\right)\right]$

mit $A=A_{1}+A_{2}$ und $B=A_{1}-A_{2}$.

Durch trigonometrische Umformungen lässt sich Gleichung (2.10) auch in der Form

$x_{\text {hom }}(t)=C e^{-\delta t} \cos \left(\omega_{d} t-\gamma\right)$

schreiben.

Der Term $F(t)$ auf der rechten Seite der Gleichung (2.1) beschreibt die Anregung durch eine von außen aufgebrachte Kraft. Als Möglichkeiten für die Fremdanregung sind die harmonische Anregung über eine Feder, einen Dämpfer, eine rotierende Unwucht oder Fluidkräfte denkbar, die jeweils durch unterschiedliche Terme formuliert werden und verschiedene Lösungen nach sich ziehen. Um die partikuläre Lösung zu erhalten, wird gemäß der Theorie ein Ansatz vom Typ der rechten Seite angewandt.

Für den Beispielfall des Rührwerks im Leerlauf handelt es sich bei der Fremdanregung um eine rotierende Unwucht.

Die Berechnung der partikulären Lösung wird in Kapitel 2.5 vorgestellt, nachdem die Grundlagen der Rotordynamik behandelt worden sind. 


\subsection{Kontinuierlicher Schwinger}

Der punktförmige Einmassenschwinger ist ein stark idealisiertes Modell, das derart in technischen Anwendungen praktisch nicht auftritt. Demgegenüber entspricht der Kontinuums- bzw. Balkenschwinger eher der Wirklichkeit.

Abbildung 2.2 zeigt eine Skizze eines einseitig fest eingespannten Balkens, der nach Schnell, Gross, Hauger [96] bei Einwirkung moderater Kräfte, die nur kleine Deformationen im elastischen Bereich verursachen und die höchstbelasteten Fasern nicht plastifizieren, ein linear elastisches Schwingungssystem darstellt.

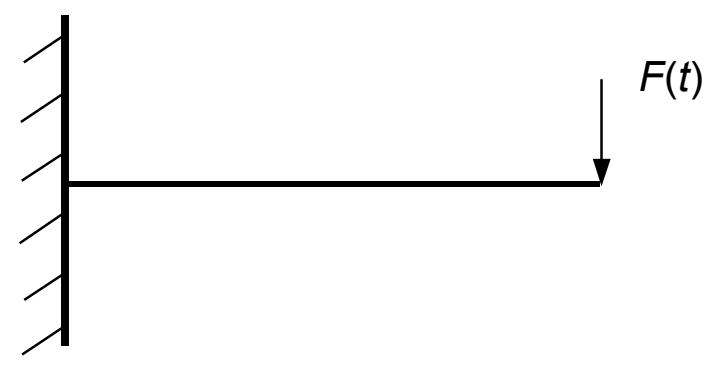

Abbildung 2.2: Skizze eines Balkenschwingers

Um hier den Zugang zur Bewegungsdifferentialgleichung zu finden, muss im Gegensatz zum Einmassenschwinger das Kräftegleichgewicht am differentiellen Element gemäß Abbildung 2.3 und nicht an der Einzelmasse betrachtet werden [80], [118]. Äußere Lasten und Materialdämpfung werden zunächst vernachlässigt.

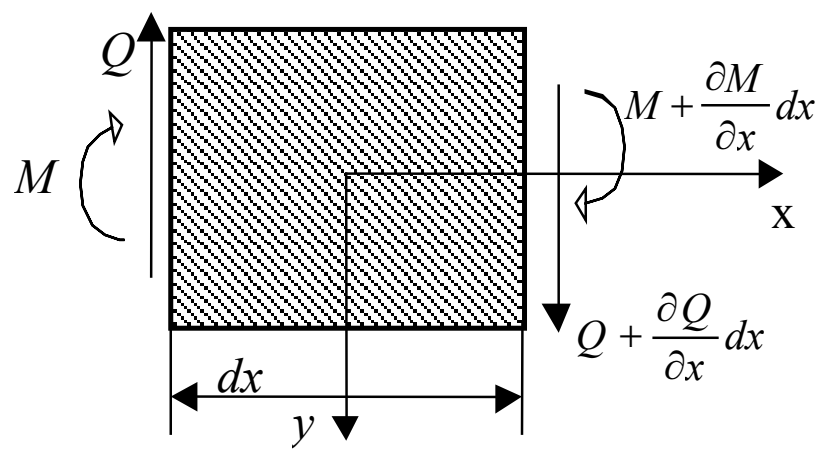

Abbildung 2.3: Kräfte am differentiellen Element 
Nach Newton gilt:

$$
m \ddot{w}=\sum F_{y}
$$

In unserem Fall bedeutet dies:

$\rho A d x \frac{\partial^{2} w}{\partial t^{2}}=-Q+Q+\frac{\partial Q}{\partial x} d x \quad$ bzw.

$\rho A \frac{\partial^{2} w}{\partial t^{2}}=\frac{\partial Q}{\partial x}$

Mit den Beziehungen aus der Elastostatik nach Schnell, Gross, Hauger [95]

$M=-E I(x) \frac{\partial^{2} w}{\partial x^{2}} \quad$ und $\quad Q=\frac{\partial M}{\partial x}$

ergibt sich:

$\frac{\partial Q}{\partial x}=\frac{\partial^{2}}{\partial x^{2}}\left(-E I(x) \frac{\partial^{2} w}{\partial x^{2}}\right)$

Nach Einsetzen dieser Beziehung in Gleichung (2.13) liefert die partielle Differentialgleichung des Kontinuumschwingers:

$\rho A \frac{\partial^{2} w}{\partial t^{2}}+\frac{\partial^{2}}{\partial x^{2}}\left(E I(x) \frac{\partial w^{2}}{\partial x^{2}}\right)=0$

Unter Annahme eines über die Länge konstanten Balkenquerschnitts und somit gleichbleibendem Flächenträgheitsmoment $(I(x)=$ konst.) kann Gleichung (2.16) folgendermaßen vereinfacht werden:

$\frac{\partial^{2} w}{\partial t^{2}}+\frac{E I}{\rho A} \frac{\partial^{4} w}{\partial x^{4}}=0$

Diese partielle Differentialgleichung lässt sich über folgenden Separationsansatz lösen:

$w(x, t)=w(x) q(t)$

Eingesetzt in Gleichung (2.17) führt dies zu nachstehender Form:

$$
\begin{aligned}
& w \ddot{q}+\frac{E I}{\rho A} w^{(4)} q=0 \quad \text { bzw. } \\
& -\frac{\ddot{q}}{q}=\frac{E I}{\rho A} \frac{w^{(4)}}{w}=\omega^{2}=\text { const. }
\end{aligned}
$$

mit $\omega=2 \pi f$

Diese Gleichung ist nur dann erfüllt, wenn die Quotienten für beliebige Werte von $q$ und $w$ den konstanten Wert $\omega^{2}$ annehmen. Durch diesen Ansatz kann Gleichung (2.19) in zwei homogene lineare Differentialgleichungen aufgetrennt werden. Sie lauten: 


$$
\begin{aligned}
& \ddot{q}+\omega^{2} q=0 \quad \text { und } \\
& w^{(4)}-\frac{\rho A}{E I} \omega^{2} w=0,
\end{aligned}
$$

die sich mit der Abkürzung

$\beta^{4}=\frac{\rho A l^{4}}{E I} \omega^{2}$

umformen lässt zu

$$
w^{(4)}-\frac{\beta^{4}}{l^{4}} w=0 \text {. }
$$

Die allgemeinen Lösungen der Differentialgleichungen lauten:

$$
q(t)=A \sin (\omega t)+B \cos (\omega t)
$$

und

$$
w(x)=C_{1} \sin \left(\frac{\beta}{l} x\right)+C_{2} \cos \left(\frac{\beta}{l} x\right)+C_{3} \sinh \left(\frac{\beta}{l} x\right)+C_{4} \cosh \left(\frac{\beta}{l} x\right)
$$

Die Konstanten $A$ und $B$ werden über die zeitlichen Anfangsbedingungen $w(t=0)$ und $\dot{w}(t=0)$ bestimmt, während sich die Konstanten $C_{1}-C_{4}$ über die kinematischen Randbedingungen ermitteln lassen.

Im Falle eines einseitig fest eingespannten Balkens werden folgende Randbedingungen angesetzt:

1. $w(x=0)=0$

2. $\dot{w}(x=0)=0$

3. $M(x=l)=-E I w^{\prime \prime}(l)=0$ bzw. $w^{\prime \prime}(l)=0$

4. $Q(x=l)=-E I w^{\prime \prime \prime}(l)=0 \quad$ bzw. $\quad w^{\prime \prime \prime}(l)=0$

Mit den Randbedingungen aus 1. und 2. erhält man:

$w(0)=C_{1} \sin 0+C_{2} \cos 0+C_{3} \sinh 0+C_{4} \cosh 0=0$

bzw.

$C_{2}+C_{4}=0$

$w^{\prime}(0)=C_{1} \frac{\beta}{l} \cos -C_{2} \frac{\beta}{l} \sin 0+C_{3} \frac{\beta}{l} \cosh 0+C_{4} \frac{\beta}{l} \sinh 0=0$

bzw.

$C_{1}+C_{3}=0$

Mit diesem Ergebnis vereinfacht sich Gleichung (2.24) zu: 


$$
w(x)=C_{1}\left[\sin \left(\frac{\beta}{l} x\right)-\sinh \left(\frac{\beta}{l} x\right]+C_{2}\left[\cos \left(\frac{\beta}{l} x\right)-\cosh \left(\frac{\beta}{l} x\right)\right]\right.
$$

Die weiteren Randbedingungen an der Stelle $x=l$ liefern:

$$
w^{\prime \prime}(l)=C_{1} \frac{\beta^{2}}{l^{2}}[\sin (\beta)+\sinh (\beta)]+C_{2} \frac{\beta^{2}}{l^{2}}[+\cos (\beta)+\cosh (\beta)]=0
$$

und

$$
w^{\prime \prime \prime}(l)=C_{1} \frac{\beta^{3}}{l^{3}}[\cos (\beta)+\cosh (\beta)]+C_{2} \frac{\beta^{3}}{l^{3}}[-\sin (\beta)+\sinh (\beta)]=0
$$

Gleichung (2.28) und (2.29) stellen ein lineares Gleichungssystem für $C_{1}$ und $C_{2}$ dar. Es gilt:

$$
\left(\begin{array}{cc}
\sin \beta+\sinh \beta & \cos \beta+\cosh \beta \\
\cos \beta+\cosh \beta & -\sin \beta+\sinh \beta
\end{array}\right)\left(\begin{array}{l}
C_{1} \\
C_{2}
\end{array}\right)=\left(\begin{array}{l}
0 \\
0
\end{array}\right)
$$

Für dieses Gleichungssystem ist eine nichttriviale Lösung für $C_{1}$ und $C_{2}$ nur dann möglich, wenn

$$
\operatorname{det}\left(\begin{array}{cc}
\sin \beta+\sinh \beta & \cos \beta+\cosh \beta \\
\cos \beta+\cosh \beta & -\sin \beta+\sinh \beta
\end{array}\right)=0
$$

gilt.

Somit folgt:

$\operatorname{det}\left(\begin{array}{cc}\sin \beta+\sinh \beta & \cos \beta+\cosh \beta \\ \cos \beta+\cosh \beta & -\sin \beta+\sinh \beta\end{array}\right)=[\sin \beta+\sinh \beta] \cdot[-\sin \beta+\sinh \beta]-$ $-[\cos \beta+\cosh \beta] \cdot[\cos \beta+\cosh \beta]=-\sin ^{2} \beta+\sin \beta \sinh \beta-\sin \beta \sinh \beta$

$+\sinh ^{2} \beta-\cos ^{2} \beta-\cos \beta \cosh \beta--\cos \beta \cosh \beta-\cosh ^{2} \beta \stackrel{!}{=} 0$

Nach Zusammenfassen ergibt sich die Beziehung

$$
1+\cos \beta \cosh \beta=0
$$

Gleichung (2.33) ist die Eigenwertgleichung für $\beta$ des einseitig fest eingespannten Kontinuumschwingers.

Aus Abbildung 2.4 geht hervor, dass eine unendliche Anzahl von Eigenwerten existiert. Die Lösung dieser Gleichung liefert für die ersten vier Eigenwerte:

\begin{tabular}{|c|c|c|c|c|}
\hline $\boldsymbol{i}$ & $\mathbf{1}$ & $\mathbf{2}$ & $\mathbf{3}$ & $\mathbf{4}$ \\
\hline $\boldsymbol{b}$ & 1,875 & 4,694 & 7,855 & 10,996 \\
\hline
\end{tabular}

Tabelle 2.1: Eigenwerte für den Kontinuumschwinger 
Mit der in Gleichung (2.33) gegebenen Definition für $\beta$ lassen sich die Eigenfrequenzen berechnen.

Aus

$\beta^{4}=\frac{\rho A}{E I} l^{4} 4 \pi^{2} f^{2}$

ergibt sich

$f=\frac{\beta^{2}}{2 \pi l^{2}} \sqrt{\frac{E I}{\rho A}}$.

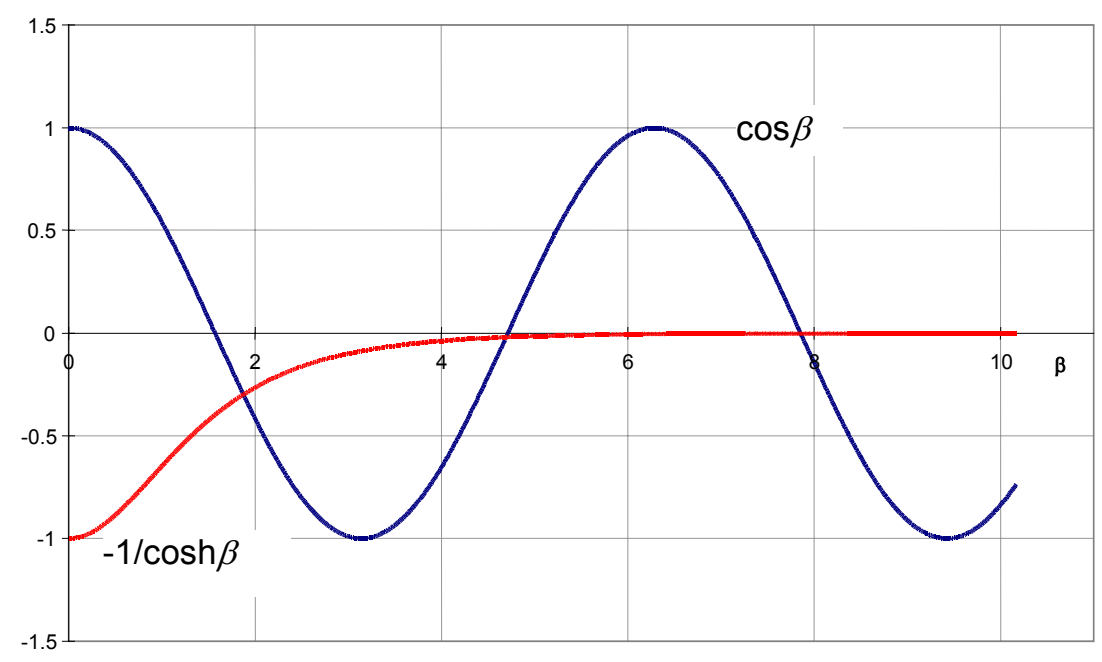

Abbildung 2.4: Darstellung der Eigenwertgleichung $\cos \beta=\frac{-1}{\cosh \beta}$ für den einseitig fest eingespannten Balkenschwinger 


\subsection{Grundlagen der Rotordynamik}

\subsubsection{Unwuchtanregung}

Bei Maschinen mit rotierenden Massen sind Unwuchten eine häufige Quelle für Schwingungen. Unwuchten treten auf, wenn die Massenverteilung im Rotor nicht symmetrisch zur Wellenachse ist. Sie entstehen bei der Fertigung durch Maßabweichungen, Materialinhomogenitäten und Exzentrizitäten zwischen Lagerstellen und dem Rotorkörper [111], [114]. Die aus Unwuchten resultierenden Fliehkräfte können sowohl im Leerlauf als auch in Fluiden zu kritischen Beanspruchungen führen [21].

Zur Betrachtung der Bewegung wird nach Gasch, Pfützner [37] ein anfänglich festes rechtwinkliges Koordinatensystem gemäß Abbildung 2.5 eingeführt, dessen $x$-Achse in Richtung der Wellenachse zeigt, während die $y$ - und $z$-Achse eine Ebene auf Höhe des Schwerpunkts des Rührerkopfes aufspannt, auf den auch die Wellenmasse reduziert wird.

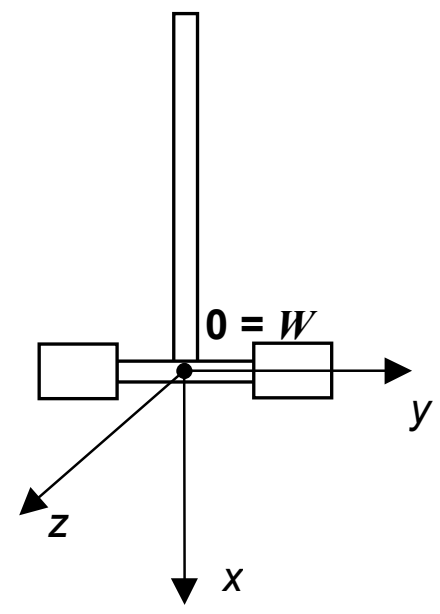

Abbildung 2.5: Lage des anfänglich festen Koordinatensystems

In der statischen Ruhelage bei nicht ausgelenkter Welle fällt der Koordinatenursprung 0 mit dem geometrischen Wellenmittelpunkt $W$ zusammen, der in der Mitte der Wellenachse liegt. Der Schwerpunkt $S$ des Gesamtsystems stimmt in der Realität nicht exakt mit dem Koordinatenursprung überein, da aufgrund fertigungsbedingter Toleranzen oder beispielsweise durch unsymmetrische Befestigung des Rotors an der Welle eine Unwucht bleibt. Die Lage der einzelnen Punkte in der $y$-z-Ebene ist in Abbildung 2.6 skizziert. Die Größenverhältnisse sind zur besseren Übersicht nicht maßstabsgetreu wiedergegeben. 


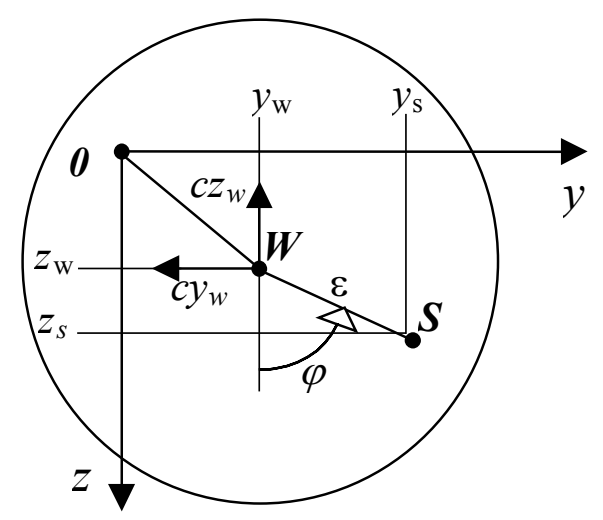

Abbildung 2.6: Größenverhältnisse in der Schwerpunktsebene

Bei Drehung der Rührerwelle erfährt diese gemäß der Lösung der Bewegungsdifferentialgleichung (2.1) eine Auslenkung, wodurch der Wellenmittelpunkt $\mathrm{W}$ gegenüber dem Koordinatenursprung verschoben liegt.

Der Schwerpunkt $S$ des Gesamtsystems weist vom Wellenmittelpunkt einen festen Abstand $\varepsilon$ auf, der als Exzentrizität bezeichnet wird, und die Koordinaten $y_{s}$ und $z_{s}$ besitzt.

Die Differentialgleichung der Translationsbewegung erhält man wiederum aus dem Kräftegleichgewicht

Trägheitskraft $=\sum$ angreifender Kräfte.

Die Bewegungsgleichungen in $y$ - und $z$-Richtung lauten

$m \ddot{y}_{s}=-c y_{w}-\dot{y}_{w} \quad$ und

$m \ddot{z}_{S}=-c z_{w}-\dot{\xi}_{w}$

mit den auf den Koordinatenursprung gerichteten elastischen Rückstellkräften $-c y_{w}$ und $-c z_{w}$ sowie den Dämpfungskräften, die über die Strukturdämpfungskonstante $\varsigma$ die Materialdämpfung berücksichtigen.

Da nur die Wellenauslenkungen $y_{w}$ und $z_{w}$ messtechnisch erfassbar sind, werden die Schwerpunktsbeschleunigungen $\ddot{y}_{S}$ und $\ddot{z}_{S}$ über $\ddot{y}_{w}$ und $\ddot{z}_{w}$ ausgedrückt.

Somit muss folgende Beziehung aufgestellt werden:

$$
\begin{aligned}
& y_{S}=y_{w}+\varepsilon \sin \varphi \\
& z_{S}=z_{w}+\varepsilon \cos \varphi
\end{aligned}
$$

Nach zweimaligem Ableiten folgt:

$$
\begin{aligned}
& \ddot{y}_{S}=\ddot{y}_{w}+\varepsilon \ddot{\varphi} \cos \varphi-\varepsilon \dot{\varphi}^{2} i n \varphi \\
& \ddot{z}_{S}=\ddot{z}_{w}-\varepsilon \ddot{\varphi} \sin \varphi-\varepsilon \dot{\varphi}^{2} \cos \varphi
\end{aligned}
$$


Unter Annahme einer konstanten Drehzahl $\dot{\varphi}=$ const. $=\Omega$ folgt daraus $\ddot{\varphi}=0$ und $\varphi=\Omega t+\gamma . \gamma$ ist dabei eine irrelevante Konstante, die durch geeignete Wahl der Randbedingung $(\varphi(t=0)=0)$ zu Null wird. In den anschließenden Rechnungen wird $\gamma=0$ angenommen.

Die folgenden Ausführungen behandeln die Berechnung der Bewegungsgleichung in $z$-Richtung, die Ergebnisse in $y$-Richtung resultieren aus analoger Betrachtung. Sie stimmen formelmäßig mit denen der z-Richtung überein.

Durch Einsetzen von Gleichung (2.40) in (2.36) lautet die Bewegungsgleichung: $m \ddot{z}_{w}-m \varepsilon \Omega^{2} \cos (\Omega t)=-c z_{w}-\dot{z}_{w} \quad$ bzw.

$m \ddot{z}_{w}+\dot{z}_{w}+c z_{w}=m \varepsilon \Omega^{2} \cos (\Omega t)$

Diese Gleichung ist identisch mit Gleichung (2.1). Die Anregungskraft $F(t)$ ist im Fall des Rührwerks eine rotierende Unwucht, die aus der exzentrischen Lage des Massenschwerpunkts resultiert.

Durch Lösung der inhomogenen Differentialgleichung erhält man die Schwingungsgleichung, welche die Bewegung des Wellenmittelpunkts nach dem Einschwingvorgang aufgrund der Erregung durch die Unwuchtkraft beschreibt.

Mit den Abkürzungen aus Kapitel 2.1 lautet Gleichung (2.41):

$$
\begin{aligned}
& \ddot{z}_{w}+2 \delta \dot{z}_{w}+\omega^{2} z_{w}=\varepsilon \eta^{2} \omega^{2} \cos (\Omega t) \quad \text { bzw. } \\
& \frac{\ddot{z}_{w}}{\omega^{2}}+\frac{2 D}{\omega} \dot{z}_{w}+z_{w}=\varepsilon \eta^{2} \cos (\Omega t) \\
& \text { mit } \eta=\frac{\Omega}{\omega} \text {. }
\end{aligned}
$$

Die partikuläre Lösung $z_{w p}$ von Gleichung (2.42) wird mit einem Ansatz vom Typ der rechten Seite unter Berücksichtigung der Phasenverschiebung $\theta$ zwischen Anregung und Ausschlag berechnet.

Daraus folgt:

$$
z_{w p}=\varepsilon V \cos (\Omega t-\theta)
$$

Durch trigonometrische Beziehungen lässt sich Gleichung (2.43) zu

$$
z_{w p}=\varepsilon V(\cos (\Omega t) \cos \theta+\sin (\Omega t) \sin \theta)
$$

umformen, woraus sich die Gleichungen

$$
\begin{aligned}
& \dot{z}_{w p}=\varepsilon V \Omega(-\sin (\Omega t) \cos \theta+\cos (\Omega t) \sin \theta) \text { und } \\
& \ddot{z}_{w p}=\varepsilon V \Omega^{2}(-\cos (\Omega t) \cos \theta-\sin (\Omega t) \sin \theta)
\end{aligned}
$$

ableiten lassen. 
Einsetzen der Gleichungen (2.44) bis (2.46) in (2.42) liefert:

$$
\begin{aligned}
& \frac{\varepsilon V \Omega^{2}}{\omega^{2}}(-\cos (\Omega t) \cos \theta-\sin (\Omega t) \sin \theta)+\frac{2 D \varepsilon V \Omega}{\omega}(-\sin (\Omega t) \cos \theta+\cos (\Omega t) \sin \theta)+ \\
& +\varepsilon V(\cos (\Omega t) \cos \theta+\sin (\Omega t) \sin \theta)=\varepsilon \eta^{2} \cos (\Omega t)
\end{aligned}
$$

Durch Zusammenfassung und Kürzen obiger Beziehung unter Berücksichtigung von $\eta=\frac{\Omega}{\omega}$ ergibt sich die Gleichung

$\left(-V \eta^{2} \cos \theta+2 D V \eta \sin \theta+V \cos \theta-\eta^{2}\right) \cos (\Omega t)+$

$\left(-V \eta^{2} \sin \theta-2 D V \eta \cos \theta+V \sin \theta\right) \sin (\Omega t)=0$

bei der beide Klammerausdrücke Null ergeben müssen, um die Gleichung zu jedem beliebigen Zeitpunkt t zu erfüllen.

Somit muss

$V\left(-\eta^{2} \cos \theta+2 D \eta \sin \theta+\cos \theta\right)=\eta^{2}$

$-\eta^{2} \sin \theta-2 D \eta \cos \theta+\sin \theta=0$

gelten.

Aus Gleichung (2.50) lässt sich die Phasenverschiebung $\theta$ (auch Phasen-Frequenzgang genannt) berechnen.

$$
\tan \theta=\frac{2 D \eta}{1-\eta^{2}}
$$

In Abbildung 2.7 ist die Phasenverschiebung über der normierten Drehzahl $\eta$ mit D als Parameter aufgetragen [38]. Hier zeigt sich, dass für $\eta=1$ unabhängig vom Parameter $D$ der Wellenmittelpunkt in jedem Fall dem Schwerpunkt um $\pi / 2$ nachläuft. Im Fall $\eta<1$ ist die Phasenverschiebung für $D=0$ Null, für $D>0$ nähert sie sich je nach Größe von $D$ in Kurven unterschiedlicher Krümmung dem Wert $\pi / 2$ für $\eta=1$. Ist $\eta>1$, steigt $\theta$ bei $D=0$ sprunghaft auf $\pi$ an, für $D>0$ nähert sich $\theta$ wieder in unterschiedlich gekrümmten Kurven dem Wert $\pi$, der erst bei $\eta \rightarrow \infty$ erreicht wird. 


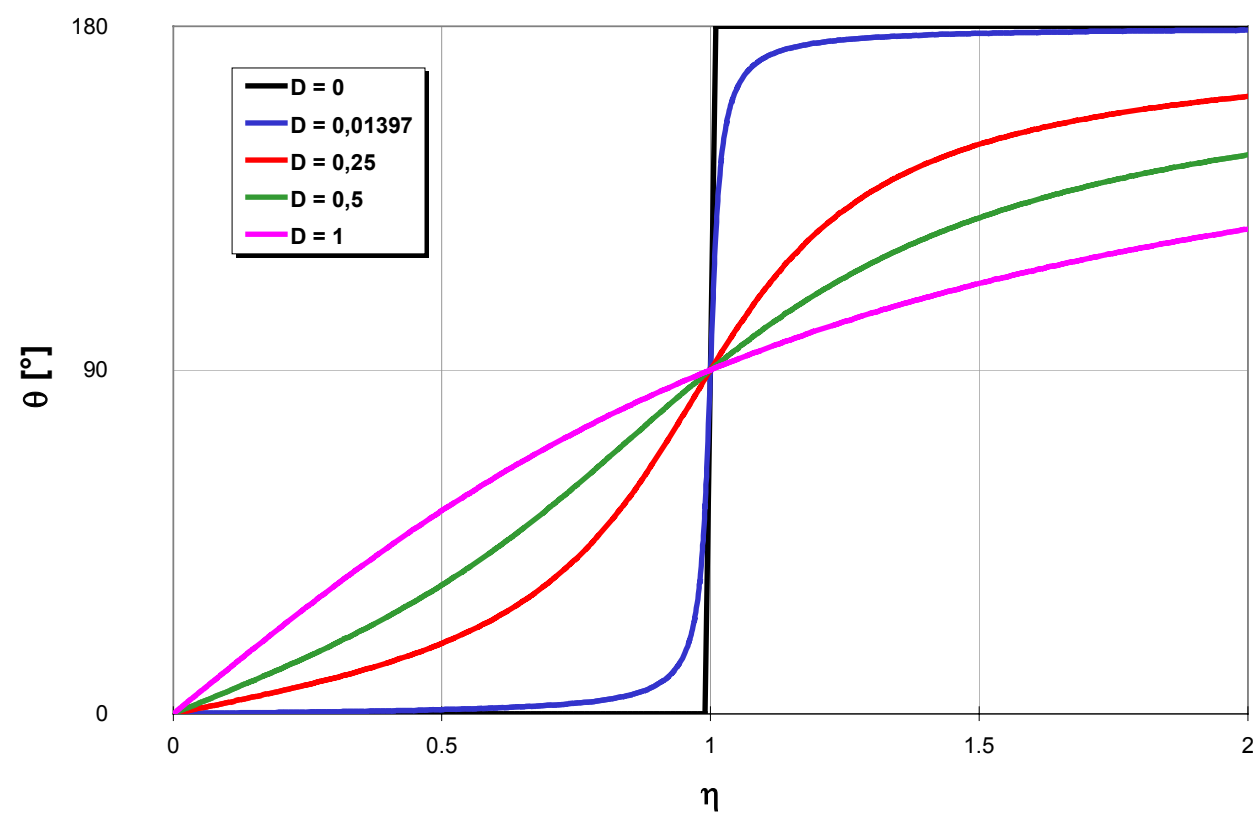

Abbildung 2.7: Phasenfrequenzgang

Unter Anwendung der trigonometrischen Beziehungen

$\sin \theta=\frac{\tan \theta}{\sqrt{1+\tan ^{2} \theta}} \quad$ und $\quad \cos \theta=\frac{1}{\sqrt{1+\tan ^{2} \theta}}$

kann nun aus Gleichung (2.49) der Verstärkungsfaktor $V$, auch als AmplitudenFrequenzgang bezeichnet, bestimmt werden. Der Verstärkungsfaktor $V$ ist ein Maß für das Verhältnis der Schwingungsamplitude zur statischen Auslenkung, die durch eine konstante Kraft verursacht wird.

Nach kurzer Rechnung folgt:

$V=\frac{\eta^{2}}{\sqrt{\left(1-\eta^{2}\right)^{2}+4 D^{2} \eta^{2}}}$

Dieser Zusammenhang ist in Abbildung 2.8 graphisch dargestellt, welche den Verstärkungsfaktor $V$ über $\eta$ (Verhältnis der Anregungsfrequenz zur Eigenfrequenz) mit $D$, dem Lehr'schen Dämpfungsmaß, als Parameter aufzeigt.

Es gilt, drei signifikante Drehzahlbereiche zu unterscheiden:

- $\quad$ unterkritischer Drehzahlbereich $(\eta<1)$

- $\quad$ kritischer Drehzahlbereich $(\eta=1)$

- $\quad$ überkritischer Drehzahlbereich $(\eta>1)$

Im unterkritischen Drehzahlbereich wächst die Wellenauslenkung zunächst mit dem Fliehkraftanteil aus der reinen Unwucht, da der Anteil der elastischen Verformungen 
des Rührwerks an der Fliehkraft noch $\mathrm{zu}$ gering ist. Im unterkritischen Drehzahlbereich liegen die Kurven von ungedämpfter und gedämpfter Betrachtung praktisch zusammen. Im Resonanzbereich treten erhebliche Abweichungen auf.

Das Maximum von $V$ bildet sich bei $\eta=1$ aus. Der theoretische Maximalwert des ungedämpften Systems liegt im Unendlichen, die Maximalwerte der gedämpften Systeme nehmen endliche Werte mit unterschiedlichen Maxima an.

Im überkritischen Drehzahlbereich nähert sich mit steigender Drehzahl der Verstärkungsfaktor dem Wert 1 an. Das bedeutet, dass der geometrische Wellenmittelpunkt bei stark überkritischer Drehzahl mit dem Abstand der Exzentrizität $\varepsilon$ um den Ursprung des Inertialsystems dreht, in dem nun der Schwerpunkt $S$ aufzufinden ist [47].

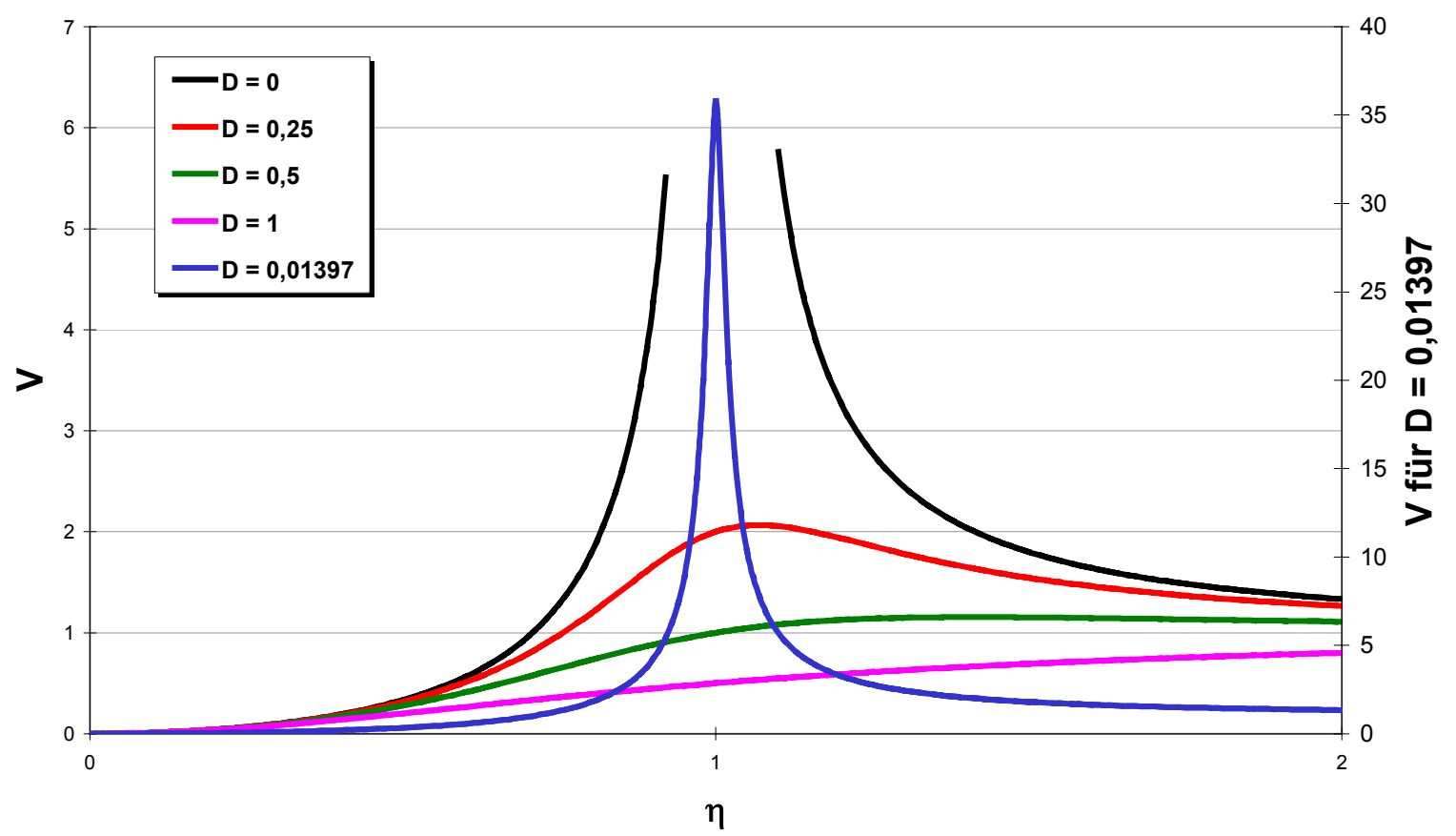

Abbildung 2.9: Amplitudenfrequenzgang

In Abbildung 2.10 ist dargestellt, wie sich die relative Lage von Schwerpunkt $S$ und geometrischem Wellenmittelpunkt $W$ mit der Drehzahl ändert. Aus der Abbildung geht hervor, dass im unterkritischen Drehzahlbereich die Schwerpunktauslenkung größer als die Auslenkung des Wellendurchstoßpunktes ist, da der Schwerpunkt durch die Fliehkräfte nach außen gezogen wird.

Das abrupte Verlagern des Schwerpunktes nach innen, das im dämpfungsfreien Fall bei $\eta=1$ auftritt, geht durch die Dämpfung in ein allmähliches „Nach-innenWandern“ mit zunehmender Drehzahl über. 
Im kritischen Drehzahlbereich $\eta=1$ stehen $0 W$ und $W S$ senkrecht aufeinander.

Im überkritischen Drehzahlbereich unterliegt das Rührwerk einer Selbstzentrierung, so dass es wieder stabil läuft [37]. Der Schwerpunkt wandert in Richtung des Ursprungs des Inertialsystems 0 .

Die Abbildung wurde aus Simulationsergebnissen mit dem in den CFD-Code implementierten numerischen Ersatzmodell für die Rührerdynamik erstellt und kann durch Gasch und Pfützner [37] und Ulbrich [112] bestätigt werden.
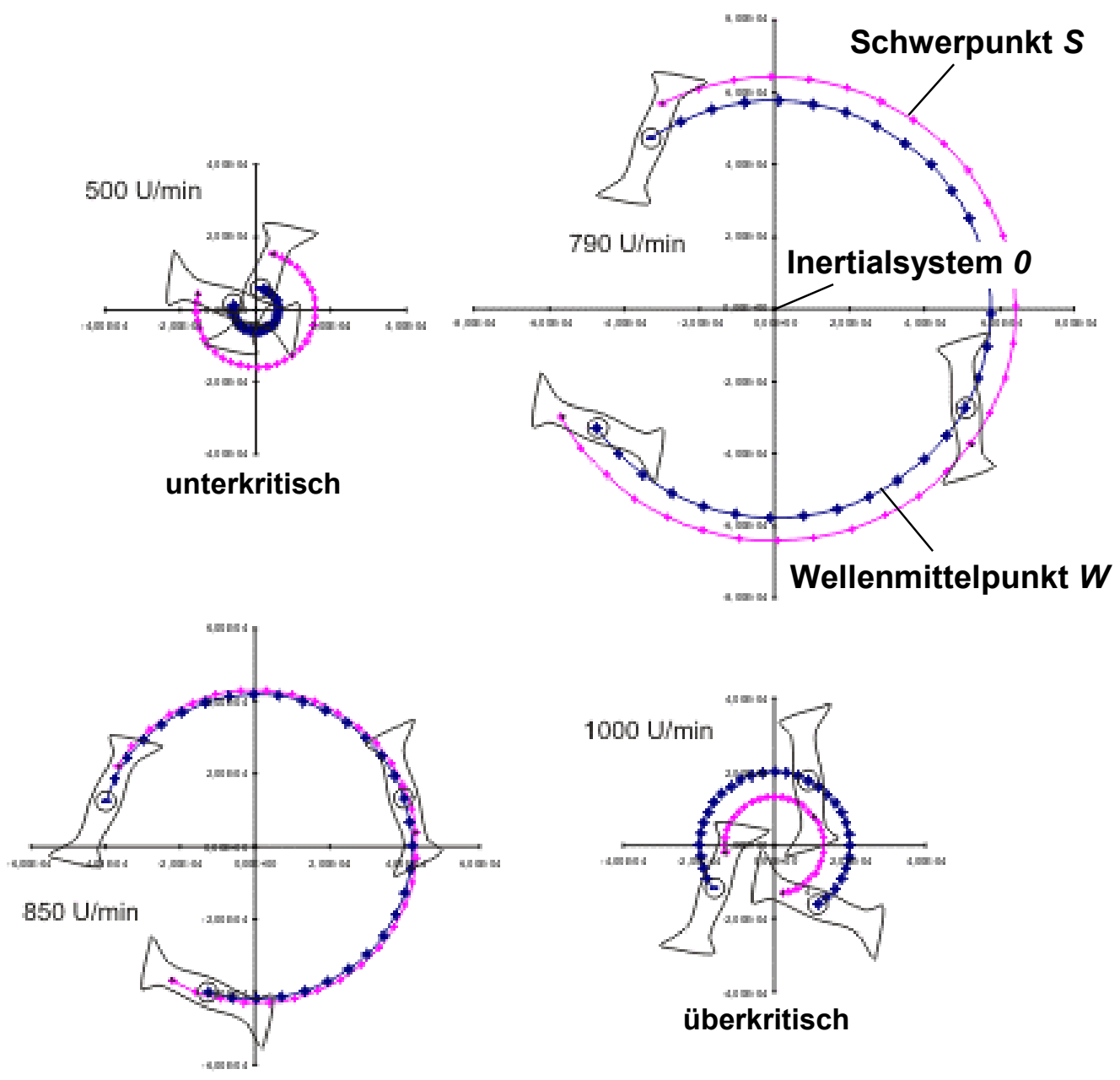

kritisch

Abbildung 2.10: Bewegungskreisbahnen vom geometrischen Mittelpunkt $W$ der Welle und des Schwerpunkts $S$ (aus numerischer Simulation) 
Schließlich erhält man durch Einsetzen von Gleichung (2.52) und (2.51) in (2.43) die Beziehung für die Auslenkung des Wellenmittelpunkts:

$$
z_{w p}=\varepsilon \frac{\eta^{2}}{\sqrt{\left(1-\eta^{2}\right)^{2}+4 D^{2} \eta^{2}}} \cos \left(\Omega t-\arctan \frac{2 D \eta}{1-\eta^{2}}\right)
$$

Aus analoger Rechnung folgt:

$$
y_{w p}=\varepsilon \frac{\eta^{2}}{\sqrt{\left(1-\eta^{2}\right)^{2}+4 D^{2} \eta^{2}}} \sin \left(\Omega t-\arctan \frac{2 D \eta}{1-\eta^{2}}\right)
$$

Der Abstand $w$ des Wellenmittelpunktes vom Ursprung berechnet sich durch geometrische Addition zu:

$$
w=\sqrt{y_{w p}^{2}+z_{w p}^{2}}=\varepsilon \frac{\eta^{2}}{\sqrt{\left(1-\eta^{2}\right)^{2}+4 D^{2} \eta^{2}}}
$$

Für die Lage der Schwerpunktkoordinaten gilt mit Gleichung (2.53) und (2.37):

$$
y_{s}=\varepsilon \cdot V \sin (\Omega t-\theta)+\varepsilon \sin (\Omega t)
$$

und

$$
z_{s}=\varepsilon \cdot V \cos (\Omega t-\theta)+\varepsilon \cos (\Omega t)
$$

Wie aus Abbildung 2.7 ersichtlich, nimmt $\theta$ bei $\eta<1$ und $D<<1$ kleine Werte an, und es lässt sich vereinfachend

$$
\begin{aligned}
& \sin (\Omega t-\theta) \approx \sin (\Omega t) \quad \text { und } \\
& \cos (\Omega t-\theta) \approx \cos (\Omega t)
\end{aligned}
$$

schreiben.

In diesem Fall vereinfachen sich die Gleichungen (2.56) bzw. (2.57) zu:

$y_{s}=\varepsilon(V+1) \sin (\Omega t)$

und

$$
z_{s}=\varepsilon(V+1) \cos (\Omega t)
$$

Unter diesen Voraussetzungen ist zu erkennen, dass die Beziehung

$$
\frac{y_{w p}}{z_{w p}}=\frac{y_{s}}{z_{s}}=\tan (\Omega t)
$$

gilt. Das bedeutet, dass der Ursprung des Inertialsystems $0, W$ und $S$ auf einer Geraden liegen und die Welle in einem stationär ausgebogenen Zustand umläuft. Das heißt, die Rührerwelle wird nur statisch beansprucht und keinen Biegewechselbeanspruchungen ausgesetzt [37]. 


\subsubsection{Fluidanregung}

Bei unsymmetrischer Anströmung des Rührwerks kann vom Rührmedium eine wesentliche Anregungskraft ausgehen.

Eine bei unsymmetrischen Rührorganen auftretende resultierende radiale Fluidkraft kann zusätzlich zur Fliehkraft wirken. Sie regt das Rührwerk mit der Drehzahl an.

Bei Rührbehältern mit Einbauten oder im Durchtrittsbetrieb können ebenfalls durch das Rührmedium induzierte, sich periodisch wiederholende Strömungsimpulse auf das Rührwerk auftreten, die mit der Eigenfrequenz des Rührwerks übereinstimmen und dieses anregen. Dieser Anregungsmechanismus tritt im unterkritischen Drehzahlbereich auf und führt dort zu erhöhten Schwingungsamplituden.

Für eine genaue Beschreibung der Fluidanregungsmechanismen wird auf Kapitel 2.5.4 verwiesen. 


\subsection{Strömungsmechanische Grundgleichungen}

Die numerische Simulation von Strömungen basiert auf physikalischen Modellen. Die mathematische Formulierung dieser Modelle resultiert in Differentialgleichungen, in denen der Transport von Strömungsgrößen beschrieben wird.

Im Folgenden werden die wichtigsten Grundgleichungen der Strömungsmechanik in kartesischen Koordinaten aufgeführt. Auf die Herleitung der einzelnen Transportgleichungen wird hier jedoch verzichtet.

\subsubsection{Massenbilanz}

Die Massenbilanz an einem ortsfesten Kontrollvolumen ergibt für die zeitliche Änderung der in dem Kontrollvolumen enthaltenen Masse (Tensorschreibweise):

$\frac{\partial \rho}{\partial t}+\frac{\partial\left(\rho u_{i}\right)}{\partial x_{i}}=0$

\subsubsection{Impulsbilanz}

Aus dem Kräftegleichgewicht an einem Fluidelement [97], [110] können die sogenannten Impulserhaltungsgleichungen abgeleitet werden, welche für reibungsbehaftete Strömungen auch Navier-Stokes-Gleichungen genannt werden. In kartesischer Tensorschreibweise erhält man für einen instationären Strömungszustand eines inkompressiblen Fluids mit konstanter Viskosität folgende Beziehung:

$$
\frac{\partial\left(\rho u_{i}\right)}{\partial t}+\frac{\partial\left(\rho u_{j} u_{i}\right)}{\partial x_{j}}=-\frac{\partial p}{\partial x_{i}}+\frac{\partial}{\partial x_{j}} \cdot\left(\mu \cdot\left(\frac{\partial u_{i}}{\partial x_{j}}+\frac{\partial u_{j}}{\partial x_{i}}-\frac{2}{3} \delta_{i j} \frac{\partial u_{k}}{\partial x_{k}}\right)\right)
$$

mit $\delta_{i j}=0$ für $i \neq j$ und $\delta_{i j}=1$ für $j=i$.

Falls die Strömungsreibung vernachlässigt werden kann $(\mu=0)$ vereinfacht sich die Impulserhaltungsgleichung zur sogenannten Euler-Gleichung:

$$
\frac{\partial\left(\rho u_{i}\right)}{\partial t}+\frac{\partial\left(\rho u_{j} u_{i}\right)}{\partial x_{j}}=-\frac{\partial p}{\partial x_{i}}
$$

Hierin sind

$$
\vec{u}=\left(\begin{array}{l}
u_{1} \\
u_{2} \\
u_{3}
\end{array}\right):=\left(\begin{array}{l}
u \\
v \\
w
\end{array}\right)
$$

der Geschwindigkeitsvektor und 


$$
\vec{x}=\left(\begin{array}{l}
x_{1} \\
x_{2} \\
x_{3}
\end{array}\right):=\left(\begin{array}{l}
x \\
y \\
z
\end{array}\right)
$$

der Ortsvektor.

Zusammen mit der Kontinuitätsgleichung (2.61) bildet Gleichung (2.62) ein geschlossenes Gleichungssystem zur Berechnung des Geschwindigkeitsfeldes $\vec{u}$ und des Druckes $p$.

\subsubsection{Allgemeine Form der Transportgleichungen}

Für Turbulenzgrößen, Enthalpie und andere skalare Komponenten existieren ebenfalls Erhaltungsgleichungen, welche eine ähnliche Form aufweisen wie die Impulserhaltungsgleichung.

Aufgrund der Ähnlichkeit dieser Erhaltungsgleichungen können diese zur allgemeinen Form der Transportgleichung zusammengefasst werden:

$$
\frac{\partial(\rho \Phi)}{\partial t}+\frac{\partial\left(\rho u_{j} \Phi\right)}{\partial x_{j}}=\frac{\partial}{\partial x_{j}}\left(\Gamma \frac{\partial \Phi}{\partial x_{j}}\right)+S_{\Phi}
$$

lok. zeitl. Änderung

Konvektion

Diffusion

Quellterm

Hierbei ist für die Variable $\Phi$ (z.B. $u, v, w, h)$ die jeweils interessierende Strömungsgröße einzusetzen. $\Gamma$ ist der dazugehörige Diffusionskoeffizient.

Alle Terme, die nicht zur Konvektion oder Diffusion gehören, werden einfach im Quellterm zusammengefasst, um eine beliebige Erhaltungsgleichung auf die Form der allgemeinen Transportgleichung zu bringen.

Die abhängige Variable $\phi$ ist hierbei eine Funktion des Ortes und der Zeit, also $\phi=\phi$ $(x, y, z, t)$.

$x, y, z$ und $t$ sind in Gleichung (2.64) die unabhängigen Variablen.

Normalerweise können alle Transportgleichungen einer Strömung in der Form der allgemeinen Transportgleichungen (2.64) dargestellt werden. 


\subsubsection{Berechnung turbulenter Strömungen}

Die meisten Rührbehälterströmungen sind bei praxisrelevanten Reynoldszahlen turbulent. Prinzipiell unterscheidet man abhängig von der Reynoldszahl $\operatorname{Re}=\frac{n \cdot d_{\text {Rührer }}^{2}}{v}$ drei Strömungsbereiche [108]:

$\operatorname{Re}<$ 10: Laminarer Strömungsbereich, der durch den Zusammenhang Ne $=\frac{P}{\rho \cdot n^{3} \cdot d_{\text {Rührer }}^{5}} \sim R e^{-1}$ charakterisiert wird.

$10<\operatorname{Re}<10^{3}$ : Übergangsbereich, in dem die laminare in eine turbulente Strömung übergeht.

$\operatorname{Re}>10^{3}: \quad$ Turbulenter Strömungsbereich, in dem die Leistungszahl Ne in der Regel konstant, d.h. unabhängig von der Reynoldszahl ist.

Durch die großen Turbulenzballen wird der Hauptströmung Energie entzogen, die beim Zerfall der Turbulenz in der sogenannten Energiekaskade zu immer kleineren Einheiten überführt wird, bis sie schließlich in Wärme zerfällt (Dissipation). Für die rechnerische Erfassung dieses Vorgangs ist es notwendig, die numerische Auflösung sowohl bezüglich des Zeitschrittes wie auch bezüglich der räumlichen Auflösung dem Zeitmaß und dem Längenmaß der kleinsten Turbulenzelemente anzupassen. Diese Diskretisierung erfordert einen unverhältnismäßig hohen numerischen Aufwand und ist auf einfache, technisch nicht relevante Strömungen beschränkt.

Die sogenannten „Direkten Simulationen“, bei denen die Navier-Stokes-Gleichungen direkt gelöst werden, werden heute für einfache turbulente Strömungen bei relativ kleinen Reynoldszahlen eingesetzt.

Bei „Grobstruktur-Simulationen“ werden die großen Turbulenzballen direkt simuliert, wohingegen die kleinen Turbulenzelemente mit Hilfe von Modellen berechnet werden. In turbulenten Strömungen sind die Transportgrößen, wie z.B. Druck und Geschwindigkeit, zeitabhängig. Um die Berechnung solcher Strömungen zu ermöglichen, ist es üblich, die Transportgrößen in einen Mittelwert und einen Schwankungsanteil aufzuteilen [23]:

$u_{i}=\bar{u}_{i}+u_{i}^{\prime}$

$p=\bar{p}+p^{\prime}$

und dann die Impulsgleichung und die Kontinuitätsgleichung über die Zeit zu mitteln: 


$$
\frac{\partial\left(\rho \bar{u}_{i}\right)}{\partial t}+\frac{\partial\left(\rho \bar{u}_{j} \bar{u}_{i}\right)}{\partial x_{j}}=-\frac{\partial \bar{p}}{\partial x_{i}}+\frac{\partial}{\partial x_{j}} \cdot\left(\mu \cdot\left(\frac{\partial \bar{u}_{i}}{\partial x_{j}}+\frac{\partial \bar{u}_{j}}{\partial x_{i}}-\frac{2}{3} \delta_{i j} \frac{\partial u_{k}}{\partial x_{k}}\right)-\rho \overline{u_{i}^{\prime} u_{j}^{\prime}}\right)
$$

sowie

$$
\frac{\partial \bar{u}_{i}}{\partial x_{i}}=0
$$

Gleichung (2.66) enthält die Reynoldsspannungen $\overline{a_{i} u_{j}}$. Diese Terme müssen durch ein Turbulenzmodell auf bekannte Größen bezogen werden, bevor eine geschlossene Lösung des Gleichungssystems möglich wird. 


\subsection{Einfluss des Fluids auf die Biegeschwingungen von Rühr- werken}

Nach Burger [21] treten an jedem Rührer, der in einem Fluid betrieben wird, mindestens vier fluiddynamische Kräfte auf, die das Biegeschwingungsverhalten des Systems Welle/Rührerkopf beeinflussen:

- Fluid- oder hydrodynamisch bedingte Trägheitskraft bzw. fluid- oder hydrodynamische Zusatzmasse

- $\quad$ Fluiddynamische Dämpfungskraft

- Fluiddynamisch bedingte Quertriebskraft

- Turbulente Strömungskräfte, die sich aus rein turbulenzbedingten Anteilen der erstgenannten drei Kräfte zusammensetzen

Die Stoffeigenschaften des Rührmediums stellen einen entscheidenden Einflussparameter auf die Biegebeanspruchung von Rührwerken dar. Das Fluid kann sowohl als Dämpfungsgröße als auch als Anregende auftreten, so dass im Vergleich zum Leerlauf sowohl kleinere als auch größere Biegebeanspruchungen resultieren können.

\subsubsection{Fluiddynamische Zusatzmasse}

Bei der Bewegung des Rührwerks wird ein Anteil des Rührgutes mitbewegt, wodurch das System Rührwerk einen Massezuwachs und somit auch einen Trägheitszuwachs erfährt. Diese mitschwingende Masse wird auch als fluiddynamische Zusatzmasse bezeichnet. Die Größe der fluiddynamischen Masse ist von den folgenden Parametern abhängig [76]:

- Größe und Bauart des Rührwerks

- $\quad$ Dichte des Rührgutes

- Geometrie des Rührbehälters

- Frequenz der Schwingungen

Nach Burger [21] wird der Anteil der Trägheitskraft, der auf die fluiddynamische Masse zurückzuführen ist, mit

$F_{\text {Träg }}=m_{\text {fluid }} \cdot \ddot{x}$

beschrieben. 
Der Einfluss der fluiddynamischen Zusatzmasse auf die kritische Drehzahl wurde bereits in Kapitel 1.3.1 ausführlich diskutiert.

\subsubsection{Fluiddämpfung}

Aufgrund der reibungsbehafteten Strömung treten vor allem am Rührerkopf Reibungskräfte auf, die eine Dämpfung der Wellenschwingung bewirken. Relevante Einflussgrößen sind auch hier die Geometrie des Rührorgans und die Stoffeigenschaften des Rührgutes.

Burger [21] berechnet die Fluiddämpfungskraft nach:

$F_{d}=-\varepsilon_{c} \cdot|\dot{x}|^{v} \cdot t^{0}$

Hierbei sind $\quad \varepsilon_{c}$ der Dämpfungskennwert, $\dot{x}$ der augenblickliche Geschwindigkeitsvektor der seitlichen Rührerauslenkung $x, \quad v \quad \operatorname{der}$ Dämpfungsexponent und $t^{0}$ der Einheitsvektor in Richtung der Bahntangente.

Der Dämpfungskennwert $\varepsilon_{c}$ hängt von der Rührgutviskosität und der Rührergeometrie $\mathrm{ab}$, wohingegen der Dämpfungsexponent lediglich durch die Rührergeometrie beeinflusst wird. Beide Werte müssen experimentell in einem Pendelversuch ermittelt werden. Für den Dämpfungsexponenten ergeben sich laut Burger [21] je nach Rührorgan Werte zwischen 0,1 und 0,5.

Gemäß Knoch [54] ist zur Bestimmung der Fluiddämpfung die Geometrie über einen geeigneten Widerstandsbeiwert $\psi$ auszudrücken, der bei komplexeren Rührergeometrien nicht vorliegt. Für die dämpfende Wirkung des Fluids ist der Strömungswiderstand in radialer Richtung relevant. Es muss also eine vereinfachte Beschreibung der Rührergeometrie gewählt werden, um die in der Literatur aufgeführten Widerstandsbeiwerte [6] einzelner einfacher Körper anwenden zu können.

Das Produkt aus Rührerdurchmesser und Blatthöhe stellt die Projektion der Seitenfläche einer Scheibe $A_{R}$ dar:

$A_{R}=d_{\text {Rührer }} \cdot h_{B}$

Der radialen Bewegung wird somit eine Dämpfungskraft

$$
F_{D}=\psi \cdot \frac{\rho}{2} \cdot w_{r e l}^{2} \cdot A_{R}
$$

entgegengesetzt. 
In den oben aufgeführten Ansätzen werden Dämpfungsbeiwerte eingesetzt, die entweder experimentell für das jeweilige Rührsystem oder über vereinfachende Geometrieannahmen bestimmt werden müssen.

Gerade im letzten Fall erscheint es fraglich, ob den an realen Rührergeometrien auftretenden Dämpfungskräften durch derart vereinfachende Annahmen ausreichend Rechnung getragen werden kann.

\subsubsection{Quertriebskraft}

Laut Burger [21] entsteht die fluiddynamische Quertriebskraft bei einer unsymmetrischen Umströmung des Rührers, wobei in diesem Kapitel nur der Primärwirbel in unmittelbarer Nähe des Rührerkopfes betrachtet werden soll. Die Achse dieses zentralen Wirbels fällt mit der Wellenachse zusammen. Wird der Rührer nun z.B. aufgrund von Unwuchtkräften ausgelenkt, so decken sich diese Achsen nicht mehr, und der Rührer wird unsymmetrisch umströmt, wodurch eine Querkraft $F_{Q}$ entsteht. Diese Quertriebskraft kann mit Hilfe der Kutta-Joukowski-Formel berechnet werden:

$$
F_{Q}=c_{\Gamma} \cdot c_{Q} \cdot \frac{\pi}{2} \cdot \rho \cdot h_{1} \cdot d_{2}^{2} \cdot[\vec{\omega} \dot{x}]
$$

Der Zirkulationsbeiwert $c_{\Gamma}$ bestimmt, in welchem Ausmaß sich die Zirkulation $\Gamma$ des Zentralwirbels an der Peripherie des Rührers ausbildet. Er ist abhängig vom Rührertyp, von den Einbauten und von der Reynoldszahl.

Der Quertriebsbeiwert $c_{Q}$ ist sowohl von der Rührerkopfgeometrie als auch von der Reynoldszahl abhängig. Zur Bestimmung des Produkts aus Zirkulationsbeiwert und Quertriebsbeiwert sind umfangreiche experimentelle Untersuchungen und Auswertungen nötig. Die fluiddynamische Quertriebskraft kann mitunter gefährliche Amplitudenzunahmen im überkritischen Drehzahlbereich hervorrufen.

\subsubsection{Turbulenzkräfte}

Gemäß Liepe [61] entstehen insbesondere bei turbulenter Umströmung des Rührers Strömungsunsymmetrien, die in einer Radialkraft $F_{r}$, Fluid auf das Rührwerk resultieren und Biegemomente verursachen.

Die an einem einzelnen Rührerblatt wirkenden Strömungskräfte setzen sich aus einem konstanten und einem fluktuierenden Anteil zusammen. Bildet man nun die Vektorsumme der angreifenden Strömungskräfte in Umfangsrichtung $F_{t i}$, ergibt sich, 
dass diese im allgemeinen ungleich Null ist. Es resultiert die hydraulische Radialkraft $F_{r, \text { Fluid }}$ (Abbildung 2.11):

$\vec{F}_{r, \text { Fluid }}=\vec{F}_{t, 1}+\vec{F}_{t, 2}+\vec{F}_{t, 3}$

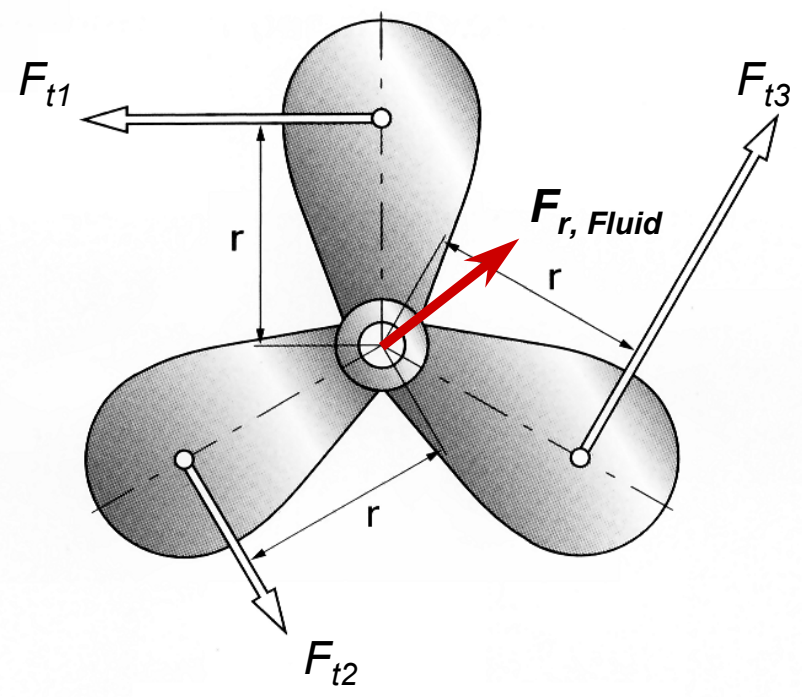

Abbildung 2.11: Hydraulische Radialkraft $F_{r, \text { Fluid }}$ als Funktion der Tangentialkräfte $F_{t, i}$

Der Summenvektor $\vec{F}_{r, \text { Fluid }}$ schwankt sowohl in Richtung als auch im Betrag, wodurch die Rührerwelle auf Biegung beansprucht wird [108]:

$M_{b}=F_{r, \text { Fluid }} \cdot l_{\text {Welle }}$

Das Gesamtbiegemoment an der Einspannstelle $M_{B}$ (Abbildung 2.12) ergibt sich somit zu [107]

$M_{B}=F_{R} \cdot l_{\text {Welle }}+\sum F_{a x, i} \cdot r$

mit der Gesamtradialkraft $F_{R}$, die sich aus radialen Anteilen der Strömung $F_{r \text {, Fluid }}$ und der Zentrifugalkraft $F_{\text {Zentrifugal }}$ zusammensetzt.

Der Biegemomentanteil $F_{a x, i} \cdot r$ aus den Axialkräften ist in der Regel klein, so dass er oft vernachlässigt wird. 


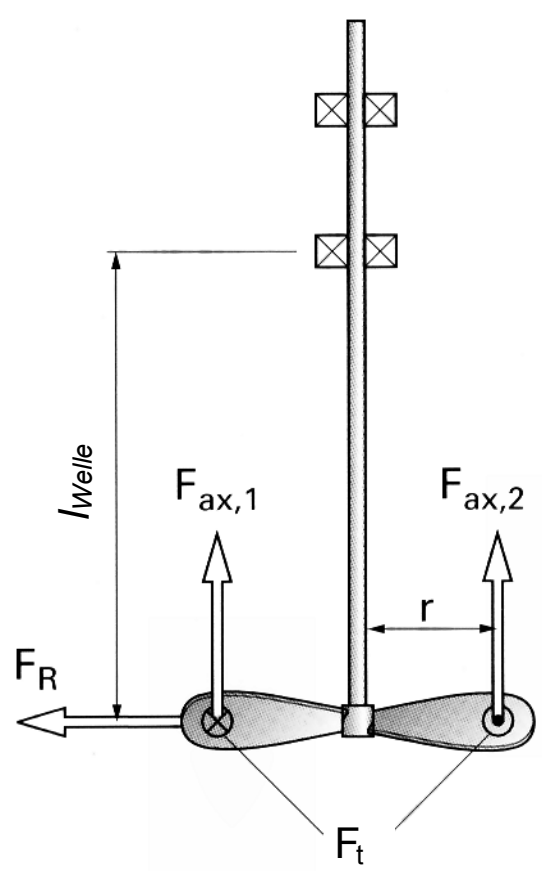

Abbildung 2.12: Axialkraft $F_{a x}$ und Gesamtradialkraft $F_{R}$

Liepe [61] untersuchte unterschiedliche Rührorgane, wobei die Ergebnisse über den Radialkraftbeiwert $c_{R}$ (siehe Gleichung (1.7)) dargestellt werden. Es werden jedoch keinerlei Aussagen über den Einfluss der Zentrifugalkraft des Rührwerks auf die Radialkraft getroffen, so dass eine Quantifizierung der Radialkraft aus rein fluiddynamischen Beanspruchungen unmöglich ist. Nur durch Vergleichsmessungen im Leerlauf kann beurteilt werden, ob der Anstieg der Kraftbeiwerte rein auf den turbulenten Strömungsanteil oder die Fliehkraft zurückzuführen ist.

Die untersuchten Propellerrührer liefern im Vergleich zu den Blattrührern kleinere Radialkraftbeiwerte.

Für radialfördernde Rührer gilt:

$c_{R}=0,45 \cdot\left(\frac{h_{1}}{d_{\text {Rührer }}}\right)^{1 / 2} \cdot K\left(N_{B}\right) \cdot c_{P}$

Dabei ist $N_{B}$ ein von der Rührerblattanzahl abhängiger Faktor.

Eine Verringerung der Turbulenz von $R e=10^{4}$ auf $R e=10^{3}$ hat eine Absenkung des Radialkraftbeiwertes um 50\% zur Folge. Diese Beobachtungen werden ebenfalls von Höhnel [43] und Fasano [32] nachgewiesen. Der Einfluss des Durchmesserverhältnisses Rührerdurchmesser $d_{\text {Rührer }} /$ Behälterdurchmesser $D$ auf den ermittelten Radialkraftbeiwert ist bei einer Anzahl von vier Strombrechern im Rührbehälter sehr gering. Beim Entleeren des Behälters treten die maximalen 
Radialkräfte auf, wenn von der Flüssigkeitsoberfläche Wirbelzöpfe bis auf das Rührorgan schlagen. Zusätzlich verstärkt werden diese Unsymmetrien im Strömungsfeld durch größere Auslenkungen des Rührers, asymmetrische Anordnung der Einbauten (z.B. Stromstörer, Abstützungen, große Einleit- bzw. Abzugsrohre), nichtzentrischen Einbau des Rührwerks im Behälter und Durchtrittsbetriebe durch die Flüssigkeitsoberfläche [116]. So wurde bei Höhnel [43] beispielsweise bei Anbringung nur eines Strombrechers in Rührernähe eine Vergrößerung des Biegebeiwertes um $80 \%$ festgestellt.

Knoch [54] beschreibt, dass auf unsymmetrisch aufgebaute Rührorgane eine resultierende radiale Fluidkraft $F_{r}$, Fluid wirkt, die mit der Drehfrequenz des Rührorgans umläuft. Sie stellt eine zusätzliche Anregungskraft mit der Anregungsfrequenz der weiterhin wirksamen Fliehkraft dar. Die Berechnung dieser fluidinduzierten Kraft erfordert jedoch detaillierte Kenntnisse über die Strömungsverhältnisse und Druckverteilungen an den einzelnen Rührerblättern und ist ohne weitere Untersuchungen zum Strömungsfeld nicht möglich.

Periodisch wirkende Strömungsimpulse auf das Rührwerk können die Eigenfrequenz des Rührwerks schon bei geringen Drehzahlen anregen und somit erhebliche Auslenkungsamplituden hervorrufen.

Pollard [81] untersuchte diese erhöhte Biegebeanspruchung im unterkritischen Drehzahlbereich und bestimmte mit der Gleichung

$f_{B}=N_{B} \cdot n$

die Blattfrequenz $f_{B}$, welche mit der Drehzahl $n$ und der Anzahl der Rührerblätter $N_{B}$ gegeben ist. Bei Übereinstimmung von Blatt- und Eigenfrequenz treten Resonanzeffekte auf, da jedes Rührerblatt, das eine kritische Stelle passiert, einen Impuls auf die Welle ausübt. Nach Höhnel [43] führt eine hohe Blattzahl zu einer Verringerung dieser fluidinduzierten Anregung, was mit einer erhöhten Dämpfung zu erklären ist. Für Blattzahlen $N_{B} \geq 6$ können die fluidinduzierten Belastungen komplett vernachlässigt werden. Auch Fasano et al. [32] ermittelten maximale Biegebeanspruchungen der Rührerwelle bei Übereinstimmung von Blatt- und Eigenfrequenz des Rührwerks. Es wird eine dimensionslose hydraulische Kraftzahl $N_{F h}$, welche die hydraulischen Kräfte auf ein Rührwerk evaluiert, durch

$N_{F h}=\frac{F_{h} \cdot d_{\text {Rührer }}}{M}$

bestimmt. 
In Abbildung 2.13 ist die hydraulische Kraftzahl $N_{F h}$ für einen vierblättrigen Schrägblattrührer und einen dreiblättrigen Hochleistungsrührer im unterkritischen Drehzahlbereich qualitativ nachgebildet.

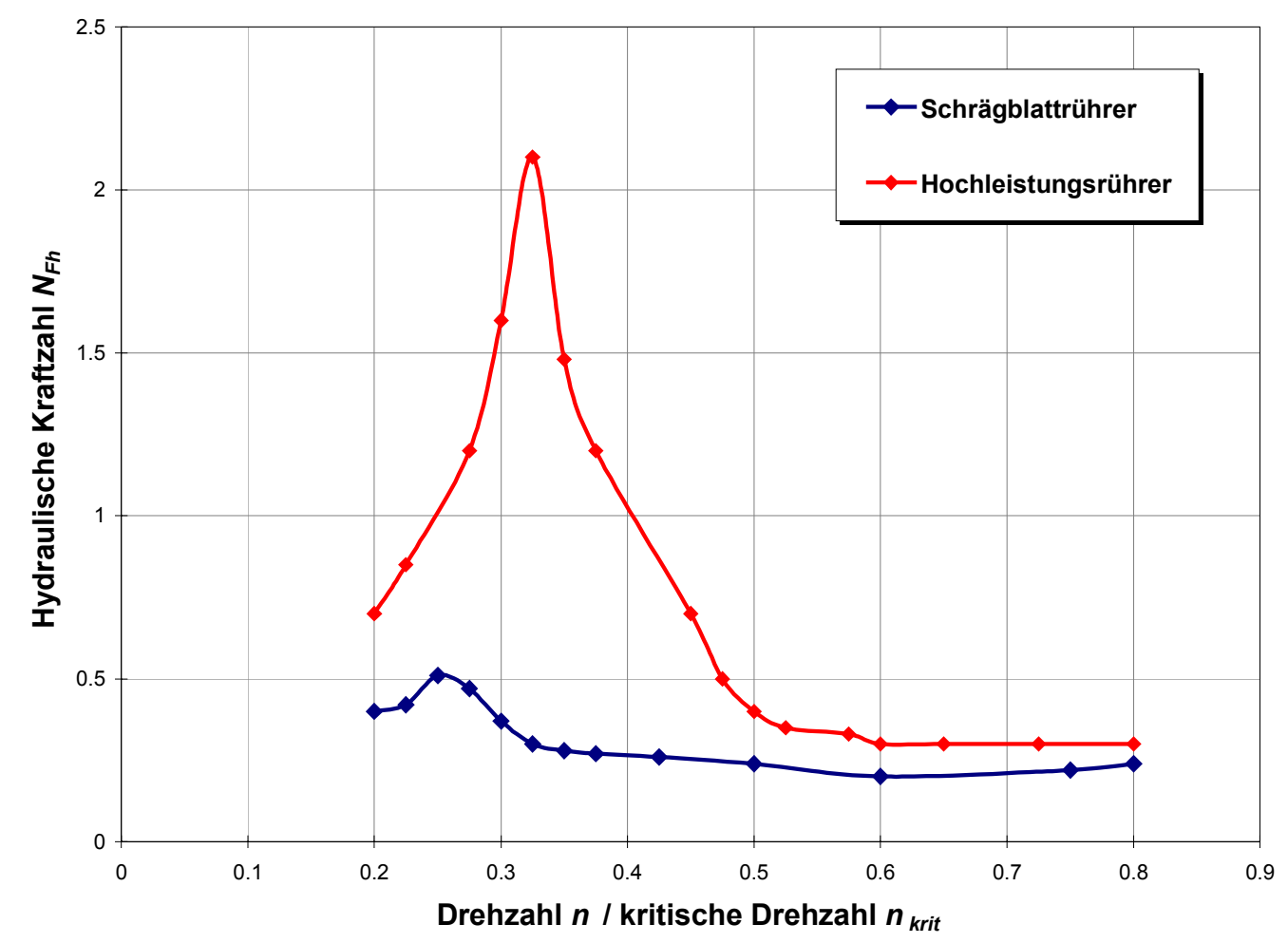

Abbildung 2.13: Hydraulische Kraftzahl nach Fasano et al. [32] mit unterkritischem Peak

Bei beiden Rührern bildet sich jeweils ein Maximum der Schwingungsamplitude aus, welches sich durch Übereinstimmung von Blattdrehzahl (siehe Gleichung (2.77)) und kritischer Drehzahl erklären lässt. Dieses Maximum (unterkritischer Peak) tritt beim vierblättrigen Schrägblattrührer bei $n_{k r i t} / n=0,25$, beim dreiblättrigen Hochleistungsrührer bei $n_{k r i t} / n=0,33$ auf.

In Kapitel 5 werden diese Untersuchungen experimentell und numerisch bestätigt und weitere Erklärungsmöglichkeiten für dieses Verhalten im unterkritischen Drehzahlbereich gefunden.

Wirbelablösungseffekte am Rührerblatt, welche ebenfalls einen Anregungsmechanismus darstellen, falls sie in Resonanz mit der Eigenfrequenz des Rührwerks liegen, können laut Fasano et al. [32] vernachlässigt werden. Sie treten bei der 2 - 12-fachen kritischen Drehzahl auf und spielen somit für die erste Eigenfrequenz keine Rolle. 


\subsection{Einfluss der Einspannsteifigkeit auf das Biegeschwingungs- verhalten von Rührwerken}

Bei Rührbehältern im technischen Maßstab ist die Befestigung der Laterne am Behälterdach keineswegs als starr anzusehen, da dieses oft nur aus dünnem Blech ohne Verstärkungen gefertigt ist. Dadurch bedingt sind größere Auslenkungen der Rührerwelle zu erwarten, die aus einer Verwölbung der Einspannung resultieren.

Fasano et al. [32] beschreiben, dass die Einspannverhältnisse zwischen Laterne und Dach einen großen Einfluss auf die kritische Drehzahl haben, welche mit abnehmender Einspannsteifigkeit merklich kleiner wird.

Statische analytische Ansätze, wie auch der von Ramsey und Zoller [82], gehen davon aus, dass die Einspannsteifigkeit des Rührwerks starr ist. In der Realität jedoch können die Einspannsteifigkeiten sehr stark variieren [104].

\subsection{Sonstige Einflüsse auf das Biegeschwingungsverhalten}

\subsubsection{Kreiseleffekt}

Aufgrund der unsymmetrischen Lagerung der freifliegenden Rührerwelle stellt sich der Rührerkopf bei einer Auslenkung um einen Winkel $\varphi$ schief, so dass infolge des vorhandenen Massenträgheitsmomentes ein Kreiselmoment auftritt. Der Einfluss des Kreiseleffekts auf die kritische Drehzahl ist bei den meisten Ausführungen von Rührwerken äußerst gering. Für ein Verhältnis von $l_{\text {Welle }} / d_{\text {Rührer }}>10$ kann überschlägig ausgesagt werden, dass der Berechnungsfehler für die kritische Drehzahl infolge Vernachlässigung des Kreiseleffektes unter 1\% liegt [115].

\subsubsection{Axialkräfte}

Axiallasten $F_{x}$ verändern die Eigenfrequenzen von Rührwerken in der Art, dass Zuglasten die Eigenfrequenzen erhöhen und Drucklasten die Eigenfrequenzen verringern. Die Veränderungen können nach Gasch und Knothe [39] erfasst werden, falls anstelle der Steifigkeiten aus Gleichung (1.9) eine korrigierte Steifigkeit

$$
c=\frac{3 E I}{l_{\text {Welle }}^{3}} \cdot\left(1+\frac{F_{x}}{F_{\text {krit }}}\right)
$$

eingesetzt wird. 
Die kritische Axiallast $F_{k r i t}$ entspricht dabei für Rührwerke der Knicklast

$$
F_{k r i t}=\frac{\pi^{2} E I}{4 l_{\text {Welle }}^{2}}
$$

des ersten Euler Falles [95]. Bei Einwirkung einer Druckkraft der Größe $F_{k r i t}$ tritt ein Knicken des Balkens auf und seine Steifigkeit wird gleich Null.

Gewöhnlich ist die Rührerwelle senkrecht von oben in den Behälter eingeführt. Somit setzen sich die Axialasten aus dem Gewicht des Rührerkopfes, dem Gewicht der Rührerwelle und den zusätzlich auftretenden Strömungslasten am Rührerkopf zusammen.

Die unter Kapitel 1.3 aufgezeigte Lösungsgleichung zur Bestimmung der kritischen Drehzahl in Luft (Gleichung (1.17)) ändert sich somit zu

$$
f_{1}=\frac{1,732}{2 \pi} \sqrt{\frac{E I}{m_{\text {Rührer }} \cdot l_{\text {Welle }}^{3}+0,236 \cdot l_{\text {Welle }}^{4} \cdot \mu}} \cdot \sqrt{1+\frac{F_{x}}{F_{\text {krit }}}}
$$

Als Axialkraft wird

$$
F_{x}=F_{G}=\left(0,5 \cdot m_{\text {Welle }}+m_{\text {Rührer }}\right) \cdot g
$$

angesetzt. Der Faktor 0,5 zur Gewichtung der Wellenmasse berücksichtigt die Tatsache, dass die Masse der Welle kontinuierlich verteilt ist und auf den Schwerpunkt bei halber Wellenlänge reduziert werden kann.

Die kritische Drehzahl wird laut Melzer [66] im Rührmedium durch die Axiallasten um ca. $10 \%$ beeinflusst.

\subsubsection{Schwingung aufgrund von Wälzlageranregung}

Nach Bäßler [4], Jamamoto [45] und Perret [79] existieren folgende Ursachen für das Zustandekommen von Biegeschwingungen bei wälzgelagerten Rotoren:

Bearbeitungsfehler sowohl der Laufbahnen von Innen- und Außenring als auch der Wälzkörper, unterschiedliche Größe der Wälzkörper und damit Verschiebung der Achsen von Innen- und Außenring, Lagerluft und der Aufbau des Wälzlagers selbst. Die ungleichförmige Abstützung infolge einer endlichen Zahl von Wälzkörpern führt $\mathrm{zu}$ einer sich periodisch ändernden Lagersteifigkeit, was wiederum zusätzliche Resonanzmöglichkeiten bedingt.

Melzer [66] stellte für unterschiedliche Lagertypen bei

$n=n_{\text {krit }} /(1,95$ bis 2,05$)$

ein signifikantes Schwingungsmaximum fest.

Ein weiteres Schwingungsmaximum bildet sich bei 


$$
n=\frac{n_{k r i t}}{n_{K a ̈ f i g} \cdot z}
$$

mit der Kugelanzahl $z$ aus, wobei die Käfigdrehzahl $n_{\text {Käfig }}$ mit

$n_{\text {Käfig }}=n \cdot \frac{D_{R, i}}{D_{R, a}+D_{R, i}}$

berechnet wird.

Bei Betrieb im Rührgut werden die wälzerregten Schwingungen aufgrund der Dämpfung des Rührmediums mehr oder minder stark unterdrückt. Die oben aufgeführten Drehzahlen sollten im Betrieb dennoch vermieden werden, was wiederum eine genaue Kenntnis der kritischen Drehzahl voraussetzt. 


\section{Numerisches Berechnungsverfahren}

\subsection{Diskretisierung der strömungsmechanischen Grundgleich- ungen}

Das Strömungsfeld wird von der verwendeten Simulationssoftware CFX-4.4 durch Lösen der Navier-Stokes-Gleichungen in einem dreidimensionalen Finite-VolumenModell bestimmt. Eine detaillierte Beschreibung der Gleichungen und des Lösungsschemas liefert das Solver Manual des CFD-Codes CFX-4.4 [101].

\subsubsection{Berechnungsgitter}

Wesentlichen Einfluss auf den Rechenzeitbedarf, die Genauigkeit und den Implementierungsaufwand übt die Wahl des Berechnungsgitters aus. Bei einem wie im CFD-Code CFX-4.4 verwendeten körperangepassten Gitter aus verzerrten Viereckselementen ist der Diskretisierungs- und Lösungsaufwand geringer, da hier durch die strukturierte Anordnung der Gitterzellen über einen Index zu jeder Gitterzelle die Nachbarzellen bekannt sind [49]. Die Rührerrotation wird mit Hilfe der „Sliding Mesh“- Technik in einem kartesischen Koordinatensystem realisiert [2].

Ein gitterunabhängiges Rührwerksverhalten wird durch Variation der Anzahl der Kontrollvolumina des Strömungsfelds überprüft. Es werden hierzu vier unterschiedliche Berechnungsgitter mit Gitterzellenanzahlen zwischen 35000 und $1 \cdot 10^{6}$ untersucht (siehe Kapitel 3.3.4).

\subsubsection{Zeitschrittweite und Iterationszahl}

Eigenen Untersuchungen [7] zufolge, sollte für reine Strömungsuntersuchungen die Anzahl der Zeitschritte (TS) pro Umdrehung $(U)$ für ein sinnvolles Ergebnis der Rührbehälterströmung nominell mindestens die Hälfte der modellierten Gitterzellen des Rührbehälters in Umfangsrichtung betragen.

Durchgeführte Mischzeituntersuchungen [7] in Rührbehältern haben ergeben, dass ca. 10 Iterationen pro Zeitschritt für eine gut auskonvergierte Lösung des Strömungsfelds notwendig sind. Im Leerlauf werden 5 Iterationen pro Zeitschritt für die Berechnung des Strömungsfeldes verwendet.

Aufgrund der zusätzlichen Betrachtung der Rührwerksdynamik wird die Zeitschrittweite in den Simulationsläufen mit dem numerischen Ersatzmodell zwischen $20 T S / U$ und $360 T S / U$, und die Iterationszahl zwischen 5 und 30 Iterationen 
pro Zeitschritt variiert. Die Ergebnisse der Parameterstudien werden in Kapitel 3.3.4 dargestellt.

\subsubsection{Räumliche Diskretisierung}

Bei der numerischen Lösung werden die differentiellen Transportgleichungen durch eine Diskretisierung in eine algebraische Form überführt.

Es gibt drei unterschiedliche numerische Diskretisierungstechniken:

- $\quad$ Finite-Differenzen

- $\quad$ Finite-Elemente

- $\quad$ Finite-Volumen

Die Grundprinzipien von Finite-Differenzen-, Finite-Elemente- und Finite-VolumenVerfahren weisen ähnliche Merkmale auf. So wird von einer ähnlichen Netzstruktur ausgegangen und die Variablen an den verschiedenen Knotenpunkten werden ähnlich verknüpft. Weiterhin entstehen bei diesen Verfahren gleichartige Matrizen, welche analog gelöst werden können [43].

\section{Finite-Volumen Methode}

Finite-Volumen Verfahren kommen primär aus dem Bereich der Strömungsmechanik, wohingegen das Finite-Element Verfahren zuerst zur Lösung von Problemen in der Feststoff- und Strukturmechanik entwickelt wurde.

Auch bei der Finite-Volumen Methode wird über das Berechnungsgebiet ein numerisches Netz gelegt. Die Differenzenquotienten in den Differentialgleichungen werden jedoch nicht direkt approximiert, sondern werden durch Integration über die einzelnen Kontrollvolumina in algebraische Gleichungen überführt. Das heißt, dass hier im Gegensatz zur Finiten-Differenzen Methode nicht die differentielle sondern die integrale Form der Transportgleichung der Ausgangspunkt der Diskretisierung ist. Die Begrenzungen der Kontrollvolumina können aus den Gitterlinien abgeleitet werden. Der zu einem Kontrollvolumen gehörende Rechenpunkt kann z.B. als der Mittelpunkt des jeweiligen Volumens bestimmt werden. Andererseits können aber auch zuerst die Knoten des Rechengitters als Rechenpunkte aufgefasst und die Kontrollvolumengrenzen jeweils in die Mitte zweier benachbarter Grenzen gelegt werden.

Mit beiden Vorgehensweisen werden sich nicht überlappende Kontrollvolumina erzeugt. 
Während die konvektierenden Größen in der Transportgleichung (2.64), also die Geschwindigkeitskomponenten $u, \quad v$ und $w$, an den einzelnen Kontrollvolumenoberflächen aus dem Ansatz der linearen Interpolation (Zentraldifferenzen-Ansatz) problemlos ermittelt werden können, tritt bei linearer Interpolation zur Bestimmung der konvektierten Größe $\phi$ eine numerische Instabilität auf. Es müssen andere Diskretisierungsansätze verwendet werden.

Es existieren eine Vielzahl von Ansätzen zur Bestimmung der Größe $\phi$ an den Kontrollvolumengrenzen [73].

Die Finite-Volumen Methode ist aus folgenden Gründen die derzeit meist verbreitete Diskretisierungsmethode bei der numerischen Simulation von Strömungsvorgängen:

- die FV Methode zeichnet sich durch eine relativ einfache Formulierung aus

- die globalen Bilanzen der konvektiven und diffusiven Flüsse werden bei der FV Methode automatisch immer richtig dargestellt

- die Quellterme können durch ein gewisses Maß an Mehraufwand konservativ diskretisiert werden

- bei der FV Methode kann auf einfache Weise die Diskretisierungsgenauigkeit erhöht werden [73]

Als Diskretisierungsfehler bzw. Lösungsfehler wird die Abweichung der Berechnungsergebnisse von einem endlich feinen zu einem unendlich feinen Gitter, das einer exakten, analytischen Lösung entsprechen würde, bezeichnet [73]. 


\section{Forderung an die Diskretisierung der Transportgleichungen}

Die wichtigste Forderung an eine Diskretisierung der Transportgleichung ist die möglichst hohe Genauigkeit. Außerdem muss eine Reihe weiterer allgemeingültiger Forderungen gestellt werden, die dafür Sorge tragen, dass die physikalische Problematik in der numerischen Formulierung möglichst gut wiedergegeben wird.

In den Transportgleichungen wird stets die Bilanz einer Strömungsgröße am infinitesimalen Kontrollvolumen und damit in der Summe auch für das gesamte betrachtete Strömungsfeld gezogen. Eine wichtige Bedingung, die an die Diskretisierung dieser Gleichungen gestellt werden muss, lautet, dass mit den aus den Diskretisierungen stammenden algebraischen Gleichungen die einzelnen Strömungsgrößen so berechnet werden, dass damit die zugehörigen integralen Bilanzen, wie z.B. die Massen-, Impuls- oder Temperaturbilanz, erfüllt werden.

Wird diese Forderung erfüllt, wird von einer „konservativen“ Diskretisierung der Transportgleichungen gesprochen.

Im Gegensatz zur Finiten-Differenzen Methode wird die Finite-Volumen Methode immer in einer „konservativen“ Diskretisierung resultieren. Allerdings muss hier eine Einschränkung getroffen werden: Bei der Finiten-Volumen Methode werden nur die konvektiven und diffusiven Terme der Transportgleichung „konservativ“ diskretisiert, wohingegen die Quellterme oftmals nichtkonservativ diskretisiert werden [73].

Den rein konvektiven Termen muss besondere Aufmerksamkeit geschenkt werden. Sie sind nichtlinear, da die gesuchten Geschwindigkeiten, quadratisch eingehen. Es wurden eine Reihe verschiedener Schemata für die Diskretisierung der konvektiven Terme entwickelt und in eigenen Arbeiten [7] vergleichend untersucht. Die Verfahren höherer Ordnung sind laut Schönung [97] erst sinnvoll, wenn ausreichend feine Berechnungsgitter verwendet werden.

In eigenen Untersuchungen zur Mischzeit in Rührbehältern [7], [10], [70], [71]wurden folgende Diskretisierungsschemata ausführlich miteinander verglichen:

- $\quad$ MIN-MOD

- $\quad$ Higher Upwind

- HYBRID

- $\quad$ QUICK

- CONDIF

- VAN LEER

- $\quad$ CCCT 


\section{- UPWIND}

Das HYBRID-Verfahren liefert bei verschiedenen Gitterfeinheiten ausreichend genaue und stabile Lösungen mit einem geringeren Rechenzeitbedarf. Aus diesem Grund wird in dieser Arbeit für alle Gitterfeinheiten das HYBRID-Verfahren [77] verwendet.

Es stellt eine Mischung aus UPWIND- und Zentraldifferenzen-Ansatz dar. Bei der Herleitung des HYBRID-Ansatzes wird von der exakten Lösung der eindimensionalen Transportgleichung ausgegangen, welche nur Konvektions- und Diffusionsterme, aber nicht die Quellterme umfasst.

Die Peclet-Zahl

$$
P e=\frac{\rho u \Delta x}{\Gamma}
$$

stellt ein Maß für das Verhältnis von konvektivem zu diffusivem Transport von $\Phi$ dar. Für $P e>2$ wird die exakte Lösung der eindimensionalen Transportgleichung durch den UPWIND-Ansatz ohne diffusiven Anteil sehr genau wiedergegeben. Bei Peclet-Zahlen kleiner zwei bietet der Zentraldifferenzen-Ansatz die exakte Näherung.

\subsubsection{Turbulenzmodellierung}

Das grundsätzliche Problem bei der Berechnung turbulenter Strömungen besteht darin, dass im Falle der zeitlichen Mittelung als zusätzliche Unbekannte der sogenannte Reynolds-Spannungstensor $\overline{\rho u_{i} u_{j}^{\prime}}$ auftritt, ohne dass weitere Gleichungen zur Verfügung stehen.

Es existieren im wesentlichen zwei verschiedene Ansätze für die Bestimmung des Reynolds-Spannungstensors [85], [117]:

\section{- Wirbelviskositätsprinzip nach Boussinesq:}

Bei der Boussinesq-Approximation werden die Reynoldsspannungen als Produkt aus einer turbulenten Wirbelviskosität $\mu_{t}$ und lokalen Strömungsgrößen ausgedrückt:

$$
-\rho \overline{u_{i}^{\prime} u_{j}^{\prime}}=\mu_{t}\left(\frac{\partial \bar{u}_{i}}{\partial x_{j}}+\frac{\partial \bar{u}_{j}}{\partial x_{i}}\right)-\frac{2}{3} \rho \delta_{i j} k
$$

mit der turbulenten kinetischen Energie

$$
k=\frac{1}{2}\left(\overline{u_{i}^{\prime} u_{i}^{\prime}}\right)
$$

Alle Turbulenzmodelle, die auf Gleichung (3.2) beruhen, können nach der 
Anzahl der zusätzlichen differentiellen Erhaltungsgleichungen eingeteilt werden, die zur Modellierung von $\mu_{t}$ gelöst werden. Demnach gibt es NullGleichungs- (algebraische Modelle) [93], Ein-Gleichungs- und ZweiGleichungsmodelle.

- Reynoldsspannungsmodelle

Die einzelnen Komponenten des Reynolds-Spannungstensors werden mit Hilfe zusätzlicher Differentialgleichungen bestimmt, die zusammen mit den Impulserhaltungsgleichungen und der Kontinuitätsgleichung gelöst werden. Im dreidimensionalen Fall führt dies auf 7 zusätzliche Differentialgleichungen (6 für die Reynolds-Spannungen und eine z.B. für die Dissipationsrate)

\section{$k-\varepsilon$ Turbulenzmodell}

Obwohl das Standard $k-\varepsilon$ Turbulenzmodell [46], [58], [59] nur isotrope Turbulenz modellieren kann, ist es das am weitesten verbreitete und verwendete Turbulenzmodell für die Simulation von Rührwerken [1], [3], [26], [29], [44], [50], [102]. Es stellt einen vernünftigen Kompromiss zwischen der physikalischen Modellierungsgenauigkeit und dem numerischen Aufwand zur Lösung der entsprechenden Gleichungssysteme dar [89]. So kann beispielsweise der Leistungseintrag eines Rührwerks mit dem $k$ - $\varepsilon$ Turbulenzmodell sehr genau bestimmt werden [31]. Auch für die im Rahmen dieser Arbeit durchzuführenden Rührersimulationen wird zunächst das $k-\varepsilon$ Turbulenzmodell verwendet.

Als Zwei-Gleichungsmodell müssen zwei zusätzliche Transportgleichungen gelöst werden, eine für die Berechnung der turbulenten kinetischen Energie und eine für die turbulente Dissipation. Aus diesen beiden zusätzlichen Variablen resultiert eine dritte Gleichung für die Bestimmung der Wirbelviskosität $\mu_{t}$. Die Wirbelviskosität $\mu_{t}$ ist keine Stoffgröße, sondern von der lokalen Turbulenzstruktur im Strömungsfeld abhängig. Sie wird aus einem Geschwindigkeitsmaß $(\alpha \sqrt{k})$ und einem Längenmaß $\left(\alpha k^{3 / 2} / \varepsilon\right)$, welches ein Maß für die Größe der Wirbel in der turbulenten Strömung darstellt, wie folgt berechnet:

$$
\mu_{t}=\rho c_{\mu} \frac{k^{2}}{\varepsilon}
$$


Dabei ist $\varepsilon$ die Dissipationsrate der turbulenten kinetischen Energie $k$, d.h. der Betrag von $k$, der pro Zeit- und Masseneinheit durch viskose Kräfte in innere Energie des Fluids umgewandelt wird:

$$
\varepsilon=\frac{\mu}{\rho} \overline{\frac{\partial u_{i}^{\prime}}{\partial x_{j}} \frac{\partial u_{i}^{\prime}}{\partial x_{j}}}
$$

Der Wert der empirischen Konstante $c_{\mu}$ kann aus Messungen wandnaher Strömungen ermittelt werden und ist in Tabelle 3.1 angegeben.

Lokale Werte von $k$ und $\varepsilon$ werden aus den Lösungen der folgenden semi-empirischen Transportgleichungen ermittelt. Für die turbulente kinetische Energie gilt:

$$
\frac{\partial(\rho k)}{\partial t}+\frac{\partial\left(\rho \bar{u}_{j} k\right)}{\partial x_{j}}=\frac{\partial}{\partial x_{j}}\left(\left(\mu+\frac{\mu_{t}}{\sigma_{k}}\right) \frac{\partial k}{\partial x_{j}}\right)+P_{k}-\rho \varepsilon
$$

Für die Dissipationsrate gilt:

$$
\frac{\partial(\rho \varepsilon)}{\partial t}+\frac{\partial\left(\rho \bar{u}_{j} \varepsilon\right)}{\partial x_{j}}=\frac{\partial}{\partial x_{j}}\left(\left(\mu+\frac{\mu_{t}}{\sigma_{\varepsilon}}\right) \frac{\partial \varepsilon}{\partial x_{j}}\right)+\frac{\varepsilon}{k}\left(c_{\varepsilon 1} P_{k}-\rho c_{\varepsilon 2} \varepsilon\right)
$$

Die Produktionsrate $P_{k}$ der turbulenten kinetischen Energie wird dabei modelliert als

$$
P_{k}=\mu_{t}\left(\frac{\partial \bar{u}_{i}}{\partial x_{j}}+\frac{\partial \bar{u}_{j}}{\partial x_{i}}\right) \frac{\partial \bar{u}_{i}}{\partial x_{j}}
$$

Die empirischen Konstanten des Turbulenzmodells sind auf die von Launder und Spalding [58] gegebenen Werte gesetzt und in der folgenden Tabelle zusammengefasst:

\begin{tabular}{||c|c|c|c|c||}
\hline$c_{\mu}$ & $\sigma_{k}$ & $c_{\varepsilon 1}$ & $c_{\varepsilon 2}$ & $\sigma_{\varepsilon}$ \\
\hline 0,09 & 1,0 & 1,44 & 1,92 & 1,3 \\
\hline \hline
\end{tabular}

Tabelle 3.1: Konstanten des Standard $k$ - $\varepsilon$ Turbulenzmodells

\section{RNG $k-\varepsilon$ Turbulenzmodell}

Das Renormalization Group (RNG) $k-\varepsilon$ Turbulenzmodell von Yakhot und Orszag [119] ist ebenfalls ein Zweigleichungsmodell, das eine Transportgleichung sowohl für die turbulente kinetische Energie als auch für die Dissipation $\varepsilon$ löst. Als Ausgangspunkt für die Modellentwicklung dienen jedoch nicht die reynoldsgemittelten Navier-Stokes-Gleichungen, sondern die der Momentanwerte. Aus diesen werden die Bewegungsgleichungen mittels der RNG-Theorie für das RNG 
$k$ - $\varepsilon$ Modell hergeleitet. Die Grundidee hierbei ist, von den Bewegungsgleichungen der Momentanwerte iterativ enge Frequenzbänder abzuspalten, um neue Gleichungen für die verbleibenden Variablen zu erhalten. Es wird diesem Verfahren zugrunde gelegt, dass die Annahme der Gleichförmigkeit der kleinen Skalen zulässig ist, d.h. Zeit- und Längenmaß reichen aus, um die kleinen Skalen im gesamten Strömungsfeld zu beschreiben. Als weitere grundlegende Annahme kommt hinzu, dass die Skalierbarkeit gegeben sein muss. Einen tieferen Einblick in die RNG-Theorie bietet Orszag [74].

Beim Vergleich mit dem Standard $k$ - $\varepsilon$-Modell fällt eine sehr große Ähnlichkeit der Modelle auf, die sich aus zwei verschiedenen Ansätzen ergeben haben. Der Hauptunterschied liegt in einem zusätzlich auftretenden Quellterm $R$ in der Dissipationsgleichung.

Der Dissipationsterm wird neben den Größen $k$ und $\varepsilon$ auch von der Scherrate beeinflusst. Dies hat eine höhere Empfindlichkeit auf Stromlinienkrümmung im Vergleich zum Standard Modell zur Folge. Die Modellkonstanten werden nicht experimentell bestimmt, sondern ergeben sich ebenfalls aus der RNG-Theorie.

\section{Reynolds-Spannungs-Modelle}

Reynolds-Spannungs-Modelle basieren im Gegensatz zu den Wirbelviskositätsmodellen zur Bestimmung des Reynolds-Spannungs-Tensors $\overline{\rho_{i} u_{j}^{\prime}}$ nicht auf der Annahme von Boussinesq. Das Schließungsproblem der Turbulenz wird auf einer höheren Ebene gelöst, indem aus den Navier-Stokes-Gleichungen eine Transportgleichung für den unbekannten Reynolds-Spannungs-Tensor abgeleitet wird [33]:

Arbeiten von Manninen und Syrjänen [63], Kohnen und Bohnet [56] und eigene Untersuchungen [7], [84] zeigen, dass der Einsatz von Reynolds-Spannungs-Modellen für Rührwerksströmungen keine Vorteile aufweist. Neben einem rechnerischen Mehraufwand durch die zusätzlich zu lösenden Gleichungen werden auch Turbulenzgrößen stärker unterschätzt als beim Einsatz des $k-\varepsilon$ Turbulenzmodells. 


\section{Randbedingungen für turbulente Strömungen}

Ein besonderes Problem bei der Verwendung von Turbulenzmodellen ist die Behandlung von Wandrandbedingungen. Im Bereich der Wand existiert eine sehr dünne laminare Unterschicht, in der die Turbulenzmodelle nicht mehr gültig sind. Diese Region muss geeignet modelliert werden.

Um die Grenzschicht mit ihren großen Gradienten auflösen zu können, muss die Knotendichte des numerischen Rechengitters entsprechend groß sein. Ebenfalls muss bei der Modellierung auf Effekte niedriger Reynoldszahlen, der sogenannten Low-Re Effekte, eingegangen werden. Dies kann bei Verwendung einer Wandfunktion vermieden werden, die eine Verbindung zwischen der Strömungsgeschwindigkeit und der Wandschubspannung herstellt. Der wandnahe Bereich wird durch empirische, logarithmische Wandfunktionen beschrieben, welche die laminare Schicht „überbrücken“ [89].

Viele statistische Turbulenzmodelle sind aufgrund ihrer Modellierung nicht in der Lage, das wandnahe Turbulenzverhalten korrekt wiederzugeben. Um dies auszugleichen, ist das Einführen von Dämpfungsfunktionen eine geeignete Möglichkeit. Auf diese Weise werden wanddämpfende Effekte und Effekte der molekularen Viskosität berücksichtigt. Die Dämpfungsfunktionen erweitern die Turbulenzmodelle für Strömungsgebiete niedriger Reynoldszahlen. Diese Art der Wandbehandlung bietet den Vorteil, die statistischen Turbulenzmodelle bis hin zur Wand verwenden $\mathrm{zu}$ können. Hierfür wurden die sogenannten Low-Re Turbulenzmodelle entwickelt. Eine Übersicht $\mathrm{zu}$ der Vielzahl an Low-Re Turbulenzmodellen findet sich in Michelassi, Rodi und Zhu [68].

Nachteil dieser Turbulenzmodelle ist der größere Speicherplatz- und Rechenzeitbedarf, da der sehr große Gradient von $\varepsilon$ in Wandnähe durch eine hohe Rechengitterauflösung aufgelöst werden muss, sobald ein Zwei-Gleichungsmodell oder höherwertigeres Modell verwendet wird.

Eine Möglichkeit, die Auflösung des Gitters in Wandnähe zu kontrollieren, ist durch die Modellgröße $y^{+}$

$$
y^{+}=\frac{\rho u_{\tau} \delta}{\mu} \text { mit } u_{\tau}=\sqrt{\frac{\tau}{\rho}}
$$

gegeben. 
Bei der Verwendung von Turbulenzmodellen mit Wandfunktionen ist darauf $\mathrm{zu}$ achten, dass die $y^{+}$Werte für die Mittelpunkte $P$ der wandnähsten Gitterzelle im Bereich $12<y^{+}<300$ liegen [24], [30], [33].

Bei Low-Re Turbulenzmodellen sollte der $y^{+}$Wert der wandnähsten Zelle kleiner 5 sein [8].

\subsubsection{Numerischer Lösungsalgorithmus}

In einem Gleichungssystem zur Beschreibung eines Strömungsproblems fehlt eine unabhängige Gleichung für den Druck, dessen Gradient in den Impulsgleichungen, für den inkompressiblen Fall jedoch nicht in der Kontinuitätsgleichung, auftritt. Die Druckbestimmung für inkompressible Medien kann durch die sogenannten Druckkorrekturverfahren gelöst werden. Hierbei werden in einem ersten Schritt die Geschwindigkeitskomponenten aus den Impulsgleichungen bestimmt. Diese werden anschließend zusammen mit dem Druck über eine Druckkorrektur korrigiert, so dass die Kontinuitätsgleichung erfüllt ist. Dies ist ein iterativer Prozess, bis sowohl Impulsgleichungen als auch Kontinuitätsgleichung erfüllt sind.

Van Doormal und Raithby [113] entwickelten das SIMPLEC-Verfahren, bei dem angenommen wird, dass die Geschwindigkeitskorrekturen an den Nachbarpunkten genauso groß sind wie am Zentralknotenpunkt. Die Druckkorrekturgleichung hat die gleiche Struktur wie beim SIMPLE-Verfahren [78], bietet jedoch den Vorteil, dass keine Unterrelaxation für den Druck benötigt wird und sich die Konvergenzrate somit erhöht. 


\subsection{Simulation der strukturmechanischen Rührerdynamik}

\subsubsection{Numerisches Ersatzmodell für die Rührerdynamik}

\section{Darstellung der Bewegungsgleichungen}

Die Differentialgleichung der Bewegung kann mit

$m \cdot \ddot{w}+\varsigma \cdot \dot{w}+c \cdot w=F_{\text {Unwucht }}+F_{C F D}$

beschrieben [96] oder in Matrixschreibweise durch die Gleichung

$\left(\begin{array}{cc}m & 0 \\ 0 & m\end{array}\right) \cdot\left(\begin{array}{l}\ddot{w}_{y} \\ \ddot{w}_{z}\end{array}\right)+\left(\begin{array}{cc}\varsigma & 0 \\ 0 & \varsigma\end{array}\right) \cdot\left(\begin{array}{l}\dot{w}_{y} \\ \dot{w}_{z}\end{array}\right)+\left(\begin{array}{cc}c & 0 \\ 0 & c\end{array}\right) \cdot\left(\begin{array}{l}w_{y} \\ w_{z}\end{array}\right)=\left(\begin{array}{l}F_{y, \text { CFD }} \\ F_{z, \text { CFD }}\end{array}\right)+\left(\begin{array}{l}F_{y, \text { Unwucht }} \\ F_{z, \text { Unwucht }}\end{array}\right)$

dargestellt werden.

Diese zeitabhängige Modellgleichung muss gelöst werden.

Im folgenden beschreibt der Begriff „numerisches Ersatzmodell“ die in den CFD-Code implementierte strukturdynamische Formulierung des Rührwerks als reduzierte Punktmasse am Rührerkopf. Die Modellbeschreibung erfolgt in diesem Kapitel.

Der Begriff „numerisches Balkenmodell“ beschreibt die in den CFD-Code implementierte strukturdynamische Formulierung des Rührwerks mit Modellierung der Rührerwelle als Euler-Bernoulli-Balken. Die Modellbeschreibung erfolgt in Kapitel 3.2.2.

\section{Modellierung von Steifigkeit und Dämpfung}

Bisher wurden in Kapitel 2.1 und Kapitel 2.2 die beiden Grenzfälle der schwingenden Punktmasse und des Balkenschwingers mit kontinuierlich verteilter Masse behandelt. Das reale System eines Rührwerks liegt gewissermaßen zwischen diesen beiden Fällen. Es setzt sich einerseits aus dem als starr betrachteten Teilsystem Rührerkopf, dessen Masse auf seinen Schwerpunkt reduziert angesehen werden kann, und andererseits aus dem Teilsystem Rührerwelle mit gleichmäßig verteilter Masse zusammen. Zunächst betrachten wir das Teilsystem Rührerkopf. Zusammen mit einer als vorerst masselos angenommenen Rührerwelle ist dieses System mit einem Einmasseschwinger vergleichbar. 


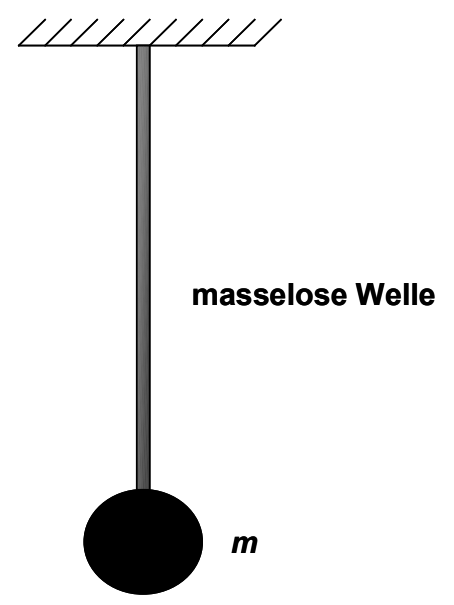

\section{Abbildung 3.1: Einmasseschwinger}

Zur Berechnung der Federsteifigkeit wird die Biegelinie des einseitig fest eingespannten Balkens bei Belastung mit einer Einzelkraft am Wellenende aus der Elastostatik nach Schnell, Gross, Hauger [95] herangezogen.

Es gilt:

$$
w(x)=\frac{F l_{\text {Welle }}^{3}}{6 E I}\left(-\frac{x^{3}}{l_{\text {Welle }}^{3}}+3 \frac{x^{2}}{l_{\text {Welle }}^{2}}\right)
$$

Für die Auslenkung am Wellenende erhält man

$$
w(l)=\frac{F l_{\text {Welle }}^{3}}{3 E I} .
$$

Mit der Definition der Federsteifigkeit $c=\frac{F}{w}$ gilt für die Federsteifigkeit einer Welle:

$$
c=\frac{F}{w}=\frac{3 E I}{l_{\text {Welle }}^{3}}
$$

Unter Berücksichtigung von Axiallasten (siehe Kapitel 2) ergibt sich eine korrigierte Steifigkeit

$$
c=\frac{3 E I}{l_{\text {Welle }}^{3}} \cdot\left(1+\frac{F_{x}}{F_{k r i t}}\right) .
$$

Bei einem in Biegeschwingung befindlichen Rührer sind als träge Massen die Masse des Rührerkopfes $m_{\text {Rührer }}$ die bereits für sich als Punktmasse betrachtet werden kann, sowie die Masse der Rührerwelle eigenfrequenzbestimmend. Die Punktmasse ist durch die Rührerkopfmasse gegeben. 
Mit der aus Gleichung (3.14) berechneten Steifigkeit $c$ ergibt sich die Eigenfrequenz eines Punktmassenschwingers $\mathrm{zu}$

$$
f_{1}=\frac{\omega}{2 \pi}=\frac{\beta^{2}}{2 \pi} \sqrt{\frac{c}{m_{\text {Rührer }}}}=\frac{\sqrt{3}}{2 \pi l^{2}} \sqrt{\frac{E I}{m_{\text {Rührer }} / l}} .
$$

In diesem Fall lautet der 1. Eigenwert $\beta=\sqrt[4]{3}$.

Um ein möglichst einfaches Modell des Gesamtsystems Rührerwelle/Rührerkopf zu erhalten, wird das Teilsystem Rührerwelle ebenfalls auf eine schwingende Einzelmasse am freien Wellenende reduziert. Da in der Realität die Wellenmasse als Streckenlast gleichmäßig über die gesamte Wellenlänge verteilt ist, wird die Wellenmasse nur anteilmäßig über den Faktor $\alpha$ in die Gleichung des Einmassenschwingers eingehen.

Die Gleichung für die 1. Eigenfrequenz des Kontinuumsschwingers unter Vernachlässigung der Rotormasse lautet:

$$
f_{1}=\frac{1,875^{2}}{2 \pi d_{\text {Welle }}^{2}} \sqrt{\frac{E I}{\rho A}}
$$

Unter Berücksichtigung des Gewichtungsfaktors $\alpha$ erhält man durch Reduktion der Wellenmasse auf einen Einmassenschwinger die Beziehung:

$$
f_{1}=\frac{\sqrt{3}}{2 \pi l_{\text {Welle }}^{2}} \sqrt{\frac{E I}{\alpha m_{\text {Welle }} / l_{\text {Welle }}}}=\frac{\sqrt{3}}{2 \pi l_{\text {Welle }}^{2}} \sqrt{\frac{E I}{\alpha \rho A}}
$$

Gleichsetzen und Umformen der Gleichungen (3.17) und (3.18) liefert

$$
\alpha=\frac{3}{1,875^{4}}=0,242 \text {. }
$$

Daraus lässt sich die auf den Schwerpunkt am Wellenende reduzierte Gesamtmasse zu

$$
m=m_{\text {Rührer }}+0,242 \cdot m_{\text {Welle }}=m_{\text {Rührer }} \cdot 0,242 \cdot \rho A l_{\text {Welle }}
$$

berechnen, wodurch sich für die 1. Eigenfrequenz des realen Systems Rührerwelle und Rührerkopf die Beziehung

$$
f_{1}=\frac{\sqrt{3}}{2 \pi d^{2}} \sqrt{\frac{E I}{\left(m_{\text {Rührer }}+0,242 \cdot \rho A l_{\text {Welle }}\right) / l}}
$$

ableitet.

Die innere, geschwindigkeitsproportionale Materialdämpfung $\varsigma$ der Rührerwelle wird über Ausschwingversuche experimentell bestimmt (siehe Kapitel 4). Hierzu muss das logarithmische Dekrement 


$$
\Lambda=\ln \left|\frac{w^{n}}{w^{n+1}}\right|
$$

aus Amplitudenmessungen zweier aufeinanderfolgender Extrema ermittelt werden. Aus dem zeitlichen Verlauf der gemessenen Reihe wird dann

$\varsigma=\frac{2 m \Lambda}{\Delta t}$

berechnet, wobei $\Delta t$ der zeitliche Abstand zweier Extrema ist. Bei großem Einfluss der Strömungskräfte kann die innere Materialdämpfung nahezu vernachlässigt werden [49].

\section{Modellierung der äußeren Lasten}

Die rechte Seite der Bewegungsgleichung (3.10) ergibt sich aus der Unwuchtkraft $F_{\text {Unwucht }}$ des Rührerkopfes und den angreifenden Strömungskräften, die mit Hilfe der numerischen Strömungssimulationssoftware CFX-4.4 bestimmt werden.

Ziel der Arbeit ist es unter anderem, die sich im Experiment bei verschiedenen Rührerdrehzahlen ergebenden Rührerauslenkungen infolge Biegung numerisch zu simulieren.

\section{Unwuchtkraft $F_{\text {Unwucht }}$}

Wie aus der Bewegungsgleichung (3.10) ersichtlich, gehen in die Amplitudenberechnung die Unwuchtkräfte $F_{\text {Unwucht }}$ maßgeblich ein. Es wird davon ausgegangen, dass die Unwuchtkraft nur vom Rührerkopf herrührt, die Welle also komplett frei von Unwucht ist. Die Unwuchtkraft hängt von den Fertigungstoleranzen des Rührerkopfes ab und kann somit nicht rein rechnerisch ermittelt werden.

Zur korrekten Simulation der Rühreramplituden kommt man also nicht umhin, bei jedem zu simulierenden Rührer vorab im Leerlauf bei unterschiedlichen geringen Drehzahlen Auslenkungsmessungen durchzuführen. Diese Drehzahlen müssen weit unterhalb der kritischen Drehzahl liegen, da dann die Zentrifugalkräfte bzw. die Rührerauslenkungen nur von den Unwuchtkräften am Rührerkopf und den elastischen Rückstellkräften abhängig sind. Bei höheren Drehzahlen sind die elastischen Verformungen $w$ des Rührwerks so groß, dass sie durch dynamische Aufschaukelung zusätzlich einen erheblichen Einfluss auf die Zentrifugalkraft haben und somit die Unwuchtkraft separat nicht mehr bestimmt werden kann. 
Es ist also möglich, für kleine Drehzahlen ein Kräftegleichgewicht zwischen Unwuchtkraft

$$
F_{\text {Unwucht }}=4 \cdot \pi^{2} \cdot n^{2} \cdot(w+\varepsilon) \cdot m
$$

und elastischen Rückstellkräften

$$
F_{\text {elast }}=c\left(w-w_{0}\right)
$$

aufzustellen, aus dem wiederum die Exzentrizität $\varepsilon$ zur zugehörigen Ersatzmasse $m$ berechnet werden kann.

$w$ ist dabei die sehr kleine Auslenkung des Rührwerks aus der idealen Nulllage und $w_{0}$ die geometrische Fehlstellung des Rührerkopfes bei Drehzahl Null, welche annähernd Null betragen wird.

Die Unwuchtkräfte in $y$ - und $z$-Richtung werden folgendermaßen in den CFD-Code implementiert [36]:

$$
\begin{aligned}
& F_{y, \text { Unwucht }}=m \cdot \varepsilon \cdot 4 \cdot \pi^{2} \cdot n^{2} \cdot \cos (2 \cdot \pi \cdot n \cdot t) \\
& F_{z, \text { Unwucht }}=m \cdot \varepsilon \cdot 4 \cdot \pi^{2} \cdot n^{2} \cdot \sin (2 \cdot \pi \cdot n \cdot t)
\end{aligned}
$$

\section{Fluidkräfte $F_{C F D}$}

Die Strömungskräfte $F_{C F D}$ werden zu jedem Zeitschritt einer CFD-Analyse

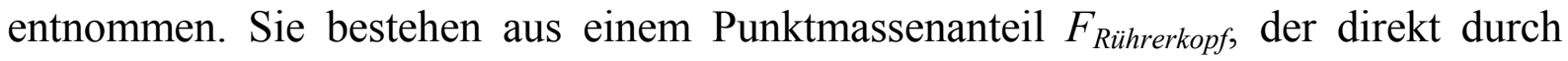
Oberflächenintegration der Drücke auf den Rührerkopf berechnet werden kann. Der andere Anteil ergibt sich aus den Oberflächendrücken entlang der Welle. Diese Fluidkräfte müssen ebenfalls auf eine Punktkraft am Wellenende reduziert werden. Zur Modellierung soll diese reduzierte Kraft dasselbe Moment am Einspannpunkt der Welle bewirken wie die Streckenlast $q$ aus dem Fluiddruck entlang der Welle. Diese Forderung ist für die spätere Betrachtung der Einspannsteifigkeit notwendig, da nur mittels dieses Ansatzes die Einspannkräfte richtig wiedergegeben werden können.

Es müssen alle $s$ Teilmomente der CFD-Patches $i$ der Breite $\Delta x_{i}$ im Abstand $x_{i}$ vom Einspannpunkt aufsummiert und auf die Wellenlänge bezogen werden (siehe Abbildung 3.2). Damit erhält man für die Strömungskräfte in $y$ - und $z$-Richtung die Modellierung:

$$
\begin{aligned}
& F_{y, \text { CFD }}=F_{y, \text { Rührerkopf }}+\frac{1}{l_{\text {Welle }}} \sum_{i=1}^{s} q_{y, i} \cdot \Delta x_{i} \cdot x_{i} \\
& F_{z, \text { CFD }}=F_{z, \text { Rührerkopf }}+\frac{1}{l_{\text {Welle }}} \sum_{i=1}^{s} q_{z, i} \cdot \Delta x_{i} \cdot x_{i}
\end{aligned}
$$




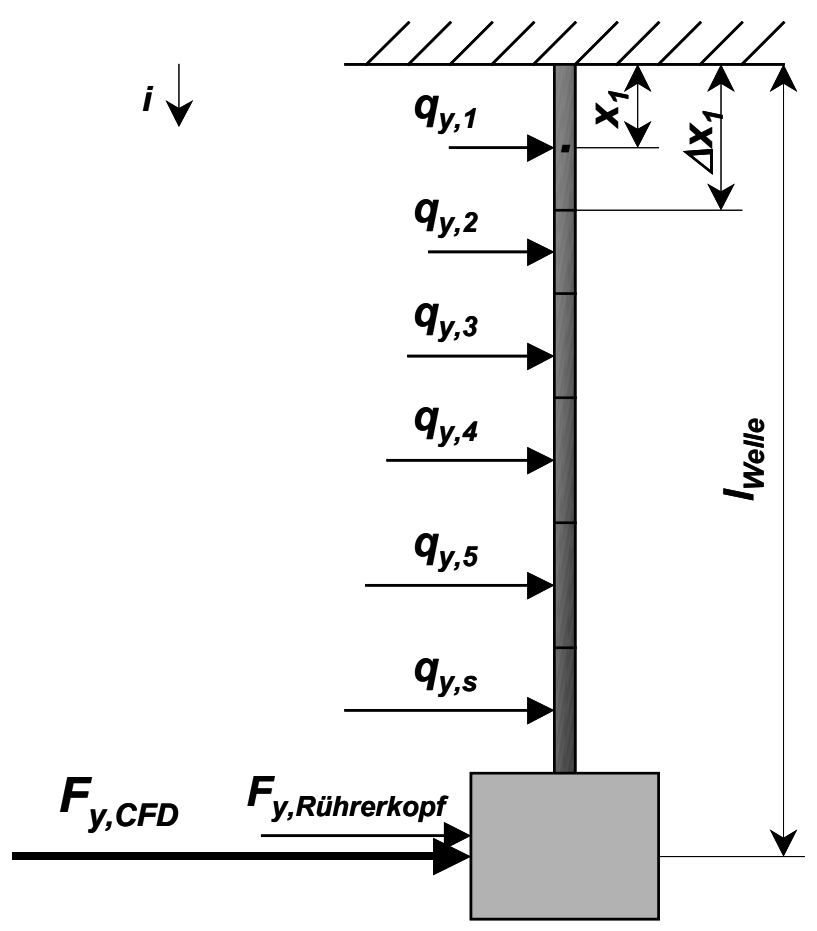

Abbildung 3.2: Veranschaulichung der Fluidkräfte beim numerischen Ersatzmodell

\section{Zeitliche Diskretisierung der Struktur}

Die Diskretisierung der Zeit ist nötig, da der kontinuierliche Verlauf einer bestimmten Größe nur an diskreten Stützstellen der Zeitkoordinate berechnet werden kann und nicht kontinuierlich bezüglich der Zeit [73].

Es gibt verschiedene Möglichkeiten der Diskretisierung, die in explizite und implizite Verfahren unterteilt werden [92].

Die expliziten Verfahren schränken die räumliche Ausbreitungsgeschwindigkeit von Informationen ein. Aus Stabilitätsgründen muss der Zeitschritt der Gitterweite angepasst werden, was häufig zu sehr kleinen Zeitschrittweiten führt und nicht effizient ist.

Bei den impliziten Verfahren sind alle Variablen der neuen Zeitebene miteinander gekoppelt. Der erhöhte numerische Berechnungsaufwand pro Zeitschritt wird durch die Möglichkeit größere Zeitschritte zu wählen meist mehr als ausgeglichen [89].

Ausgehend von der allgemeinen Schwingungsgleichung (3.10) lässt sich diese zeitliche Abfolge der Berechnungsschritte, wie in Abbildung 3.3 gezeigt, schematisch darstellen. 

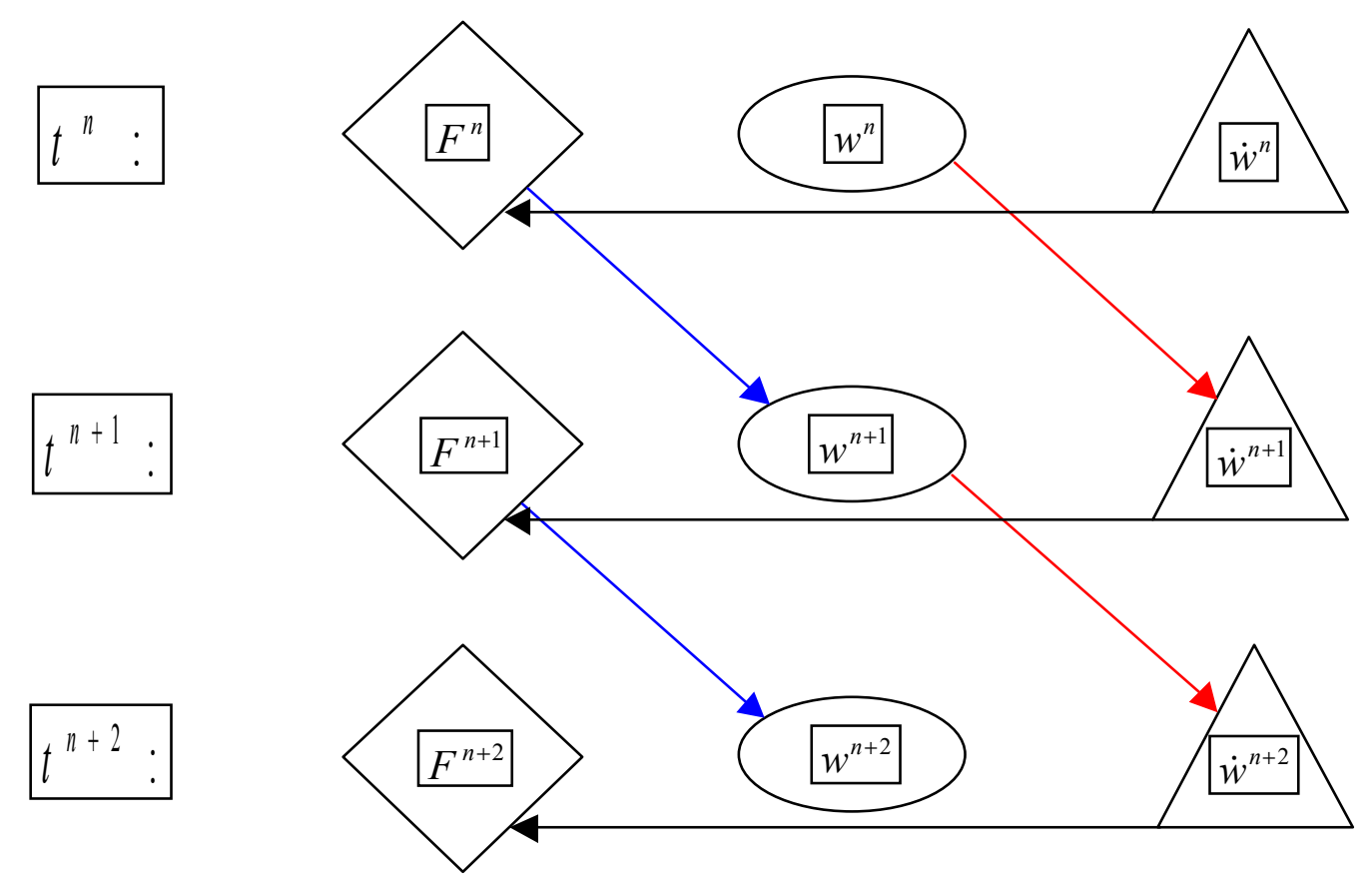

Abbildung 3.3: Schematische Darstellung der zeitlichen Abfolge der Berechnungsschritte

Die verschiedenen Verfahren der Zeitdiskretisierung unterscheiden sich nur durch die jeweiligen Ausdrücke, die für die Auslenkung $w$ und ihre erste zeitliche Ableitung $\dot{w}$ in die Gleichung eingesetzt werden. Für die zweite zeitliche Ableitung $\ddot{w}$ wird jeweils der in Gleichung (3.29) dargestellte Zusammenhang verwendet:

$$
\ddot{w}=\frac{w^{n+1}-2 w^{n}+w^{n-1}}{\Delta t^{2}}
$$

\section{Backward Differencing Formula (BDF)-Verfahren}

Die zeitliche Ableitung der Auslenkung $w$ der Welle wird im BDF-Verfahren 1.Ordnung durch Gleichung (3.30) angenähert.

$$
\dot{w}^{n+1}=\frac{w^{n+1}-w^{n}}{\Delta t}
$$

Dies ist eine zeitlich rückwärtsgerichtete Differenz, weshalb man auch von einem impliziten Verfahren spricht, da eine explizite Auflösung von Gleichung (3.30) nach $w^{n+1}$ nicht möglich ist. Dadurch sind alle Variablen der neuen Zeitebene miteinander gekoppelt, und zur Berechnung jeder neuen Zeitebene ist die Lösung eines Gleichungssystems erforderlich [89]. Mit der für ein implizites Verfahren zu verwendenden Bewegungsgleichung 
$m \ddot{w}^{n+1}+\zeta \dot{w}^{n+1}+c w^{n+1}=F^{n+1}$

folgt mit den Gleichungen (3.29) und (3.30) ein Ausdruck für $w^{n+1}$, der in den CFD Code implementiert wird:

$$
w^{n+1}=\frac{F^{n+1}+w^{n}\left(\frac{2 m}{\Delta t^{2}}+\frac{\zeta}{\Delta t}\right)-w^{n-1}\left(\frac{m}{\Delta t^{2}}\right)}{\frac{m}{\Delta t^{2}}+\frac{\zeta}{\Delta t}+c}
$$

\section{$\underline{\text { Leapfrog-Verfahren }}$}

Die Leapfrog-Methode diskretisiert den zeitabhängigen Term der Auslenkung mittels zentraler Differenzen 2. Ordnung:

$$
\dot{w}^{n}=\frac{w^{n+1}-w^{n-1}}{2 \Delta t}
$$

Hierbei handelt es sich um eine explizite Methode, die drei Zeitebenen einbezieht. Im Unterschied zu impliziten Verfahren kann die Gleichung explizit nach $w^{n+1}$ aufgelöst werden, so dass auf der rechten Seite nur Werte der bekannten Zeitebene $t^{n}$ stehen. Mit der Bewegungsgleichung für implizite Verfahren

$$
m \ddot{w}^{n}+\zeta \dot{w}^{n}+c w^{n}=F^{n}
$$

und den Gleichungen (3.29) und (3.33) ergibt sich:

$$
w^{n+1}=\frac{F^{n}+w^{n}\left(c-\frac{2 m}{\Delta t^{2}}\right)-w^{n-1}\left(\frac{m}{\Delta t^{2}}-\frac{\zeta}{2 \Delta t}\right)}{\frac{m}{\Delta t^{2}}+\frac{\zeta}{2 \Delta t}}
$$

Die in Gleichung (3.35) einzusetzende Kraft $F^{n}$ ist aus dem vorhergehenden Zeitschritt bekannt.

\section{Crank-Nicolson-Verfahren}

Ein semi-implizites Verfahren, d.h. eine Kombination einer expliziten und impliziten Diskretisierung stellt die Crank-Nicolson-Methode dar [89]. Die entsprechende Beziehung für die Diskretisierung lautet:

$$
\frac{1}{2}\left(\dot{w}^{n+1}+\dot{w}^{n}\right)=\frac{w^{n+1}-w^{n}}{\Delta t}=\dot{w}^{n+1 / 2}
$$


Die Differentialgleichung wird also mit einer zentralen Differenz zum Zeitpunkt $t^{n+1 / 2}$ diskretisiert. Durch Einsetzen der Gleichungen (3.29) und (3.36) in die für die zentrale Differenz geeignete Bewegungsgleichung

$m \ddot{w}^{n+\frac{1}{2}}+\zeta \dot{w}^{n+\frac{1}{2}}+c w^{n+\frac{1}{2}}=F^{n+\frac{1}{2}}$

ergibt sich mit

$F^{n+\frac{1}{2}}=\frac{1}{2}\left(F^{n}+F^{n+1}\right)$

und

$$
w^{n+\frac{1}{2}}=\frac{1}{2}\left(w^{n}+w^{n+1}\right)
$$

die zu implementierende Gleichung

$$
w^{n+1}=\frac{\frac{1}{2}\left(F^{n}+F^{n+1}\right)+w^{n}\left(\frac{2 m}{\Delta t^{2}}+\frac{\zeta}{\Delta t}-\frac{c}{2}\right)-w^{n-1}\left(\frac{m}{\Delta t^{2}}\right)}{\frac{m}{\Delta t^{2}}+\frac{\zeta}{\Delta t}+\frac{c}{2}}
$$

\section{$\underline{\text { Newmark-Verfahren }}$}

Das implizite Newmark-Verfahren [5], [55], [72] ist komplexer als die vorher beschriebenen Methoden. Ausgehend von der Bewegungsgleichung zum Zeitpunkt $t^{n+1}$ (Gleichung (3.31)) werden die von Newmark formulierten Zusammenhänge für konstante Beschleunigung angewendet [72], bei denen der Fehler in der Energiebilanz Null ist [55].

Das Newmark-Verfahren ist frei von numerischer Dämpfung und mit dem kleinsten Fehler in der Betrachtung der Periodenlänge behaftet.

Die Differentialgleichung der Bewegung (siehe Gleichung (3.41)) wird zum neuen Zeitschritt $n+1$ betrachtet.

$m \cdot \ddot{w}^{n+1}+\cdot \varsigma \cdot \dot{w}^{n+1}+c \cdot w^{n+1}=F^{n+1}$

Ort und Geschwindigkeit ergeben sich bei $n+1 \mathrm{zu}$

$$
\begin{aligned}
& w^{n+1}=w^{n}+\dot{w}^{n} \Delta t+\frac{1}{4} \Delta t^{2}\left(\ddot{w}^{n}+\ddot{w}^{n+1}\right) \\
& \dot{w}^{n+1}=\dot{w}^{n}+\frac{1}{2} \Delta t\left(\ddot{w}^{n}+\ddot{w}^{n+1}\right)
\end{aligned}
$$

Gleichung (3.42) wird nach $\ddot{w}^{n+1}$ aufgelöst und in die Geschwindigkeitsdefinition (3.43) für die Berechnung von $\dot{w}^{n+1}$ eingesetzt. 
Die Ausdrücke $w^{n+1}, \dot{w}^{n+1}$ und $\ddot{w}^{n+1}$ werden schließlich in die Bewegungsgleichung eingesetzt, so dass sich als Auslenkung zum neuen Zeitschritt

$$
w^{n+1}=\frac{\Delta t^{2} F^{n+1}+(4 m+2 \Delta t \varsigma) w^{n}+\left(4 \Delta t m+\Delta t^{2} \varsigma\right) \dot{w}^{n}+\Delta t^{2} m \ddot{w}^{n}}{4 m+2 \Delta t \varsigma+\Delta t^{2} c}
$$

ergibt.

Für die Implementierung in den CFD-Code wurden die Newmarkfaktoren $c=0,5$ und $b=0,25$ verwendet, da mit diesen Parametereinstellungen nach Newmark [72] die numerische Diffusion verschwindet und alle Zeitschritte stabil sind. 


\subsubsection{Modellierung der Rührerwelle als Euler-Bernoulli-Balken}

Zur Überprüfung des in Kapitel 3.3.1 vorgestellten numerischen Ersatzmodells für die Rührerdynamik wird die Rührerwelle nun als Euler-Bernoulli-Balken diskretisiert.

Es müssen hierfür einige Annahmen getroffen werden, die in der Literatur [20], [42] ausführlich beschrieben werden.

\section{Räumliche Diskretisierung}

Die differentielle Bewegungsgleichung eines Balkens mit konstantem Querschnitt unter Berücksichtigung von äußeren Lasten und Materialdämpfung lautet:

$$
m_{B} \frac{\partial^{2} w}{\partial t^{2}}+\varsigma_{B} \frac{\partial w}{\partial t}+E I \frac{\partial^{4} w}{\partial x^{4}}-F \frac{\partial^{2} w}{\partial x^{2}}-\frac{\partial F}{\partial x} \frac{\partial w}{\partial x}=q
$$

Sie ergibt sich aus dem dynamischen Kräftegleichgewicht zwischen Trägheitskräften, Querkräften, geschwindigkeitsabhängigen Dämpfungskräften und äußerer Streckenlast am infinitesimalen Element

$$
m_{B} \frac{\partial^{2} w}{\partial t^{2}}=\frac{\partial Q}{\partial x}-\varsigma_{B} \frac{\partial w}{\partial t}+q \text {. }
$$

Die äußere Streckenlast $q$ resultiert aus der Umströmung des Rührwerks und der Unwuchtkraft $F_{\text {Unwucht }}$. Die axiale Kraft $F$ ergibt sich aus Fluidlasten in Längsrichtung und dem Eigengewicht des Rührwerks. Sie wird über die Balkenlänge als konstant angenommen, was eine bedeutende numerische Vereinfachung darstellt. Die erhaltene Schwingungsgleichung gilt sowohl für die $y$-Richtung als auch für die $z$-Richtung. Obwohl heutzutage wegen ihrer flexiblen Einsatzmöglichkeiten numerische strukturdynamische Berechnungen mit Finite-Element-Programmen durchgeführt werden [5], ist im vorliegenden Fall die Formulierung der Diskretisierung der Bewegungsgleichung mit Hilfe von finiten Differenzen deutlich einfacher zu realisieren [100]. Um die Wellenbewegung numerisch bestimmen zu können, wird die Rührerwelle in $n_{x}$ äquidistante Abschnitte eingeteilt. Die Ableitungen nach dem Ort werden durch finite Differenzquotienten zweiter Ordnung ersetzt:

$$
\begin{aligned}
& w_{0}^{\prime}=\frac{w_{1}-w_{-1}}{2 \Delta x} \\
& w_{0}^{\prime \prime}=\frac{w_{1 / 2}^{\prime}-w_{-1 / 2}^{\prime}}{\Delta x}=\frac{1}{\Delta x^{2}}\left(w_{1}-2 w_{0}+w_{-1}\right) \\
& w_{0}^{\prime \prime \prime}=\frac{1}{\Delta x}\left(w_{1 / 2}^{\prime \prime}-w_{-1 / 2}^{\prime \prime}\right)=\frac{1}{\Delta x^{3}}\left(\frac{w_{2}}{2}-w_{1}+w_{-1}-\frac{w_{-2}}{2}\right)
\end{aligned}
$$


$w_{0}^{\prime \prime \prime}=\frac{1}{\Delta x}\left(w_{1 / 2}^{\prime \prime \prime}-w_{-1 / 2}^{\prime \prime \prime}\right)=\frac{1}{\Delta x^{4}}\left(w_{2}-4 w_{1}+6 w_{0}-4 w_{-1}+w_{-2}\right)$

Am Einspannrand gelten die folgenden Randbedingungen:

$$
\begin{aligned}
& w_{0}=0 \\
& w_{0}^{\prime}=0
\end{aligned}
$$

Die Randbedingungen am freien Balkenende lauten:

$$
\begin{aligned}
& M\left(n_{x}\right)=0 \Rightarrow w_{n_{x}}^{\prime \prime}=0 \\
& Q\left(n_{x}\right)=0 \Rightarrow w_{n_{x}}^{\prime \prime \prime}=0
\end{aligned}
$$

Somit ergibt sich die symmetrische Pentadiagonal-Steifigkeitsmatrix zu:

$$
\begin{aligned}
& K=\frac{E I}{\Delta x^{4}}\left(\begin{array}{ccccccccc}
7 & -4 & 1 & 0 & \cdots & \cdots & \cdots & \cdots & 0 \\
-4 & 6 & -4 & 1 & 0 & & & & \vdots \\
1 & -4 & 6 & -4 & 1 & 0 & & & \vdots \\
0 & 1 & -4 & 6 & -4 & 1 & 0 & & \vdots \\
\vdots & \ddots & \ddots & \ddots & \ddots & \ddots & \ddots & \ddots & \vdots \\
\vdots & & 0 & 1 & -4 & 6 & -4 & 1 & 0 \\
\vdots & & & 0 & 1 & -4 & 6 & -4 & 1 \\
\vdots & & & & 0 & 1 & -4 & 5 & -2 \\
0 & & & & & 0 & 2 & -4 & 2
\end{array}\right) \\
& +\frac{F}{\Delta x^{2}}\left(\begin{array}{ccccccc}
2 & -1 & 0 & \cdots & \cdots & \cdots & 0 \\
-1 & 2 & -1 & & & & \vdots \\
0 & -1 & 2 & -1 & & & \vdots \\
\vdots & \ddots & \ddots & \ddots & \ddots & \ddots & \vdots \\
\vdots & & 0 & -1 & 2 & -1 & 0 \\
\vdots & & & 0 & -1 & 2 & -1 \\
0 & & & 0 & 0 & 0 & 0
\end{array}\right)
\end{aligned}
$$

Mit der symmetrischen Diagonal-Massenmatrix 


$$
M=\left(\begin{array}{cccc}
m_{B} & 0 & \cdots & 0 \\
0 & \ddots & \ddots & \vdots \\
\vdots & \ddots & \ddots & 0 \\
0 & \cdots & 0 & m_{B}
\end{array}\right)
$$

und der symmetrischen Diagonal-Dämpfungsmatrix

$$
C=\left(\begin{array}{cccc}
\varsigma_{B} & 0 & \cdots & 0 \\
0 & \ddots & \ddots & \vdots \\
\vdots & \ddots & \ddots & 0 \\
0 & \cdots & 0 & \varsigma_{B}
\end{array}\right)
$$

erhält man schließlich für den Zeitpunkt $n+1$ die diskretisierte Bewegungsgleichung:

$$
M \ddot{\ddot{w}}^{n+1}+C \dot{\vec{w}}^{n+1}+K \vec{w}^{m+1}=\vec{q}^{n+1}
$$

\section{Zeitliche Diskretisierung und iterative Lösung des Gleichungssystems}

Die zeitliche Diskretisierung erfolgt wie schon beim numerischen Ersatzmodell für die Rührerdynamik nach der Methode von Newmark. Es ergeben sich, abhängig von der Lage des zu berechnenden Knotens folgende Lösungsformen:

- $\quad$ regulärer Knoten $i=3$ bis $n_{\max -2}$ :

$$
w_{i}^{n+1}=\frac{b_{i}^{n+1}+\Delta t^{2} K_{0}\left(-w_{i-2}^{n+1}+4 w_{i-1}^{n+1}+4 w_{i+1}^{n+1}-w_{i+2}^{n+1}\right)+\Delta t^{2} K_{1}\left(w_{i-1}^{n+1}+w_{i+1}^{n+1}\right)}{4 m_{B}+2 \Delta t \varsigma_{B}+6 \Delta t^{2} K_{0}+2 \Delta t^{2} K_{1}}
$$

- $\quad i=1$

$$
w_{1}^{n+1}=\frac{b_{1}^{n+1}+\Delta t^{2} K_{0}\left(4 w_{2}^{n+1}+4 w_{i+1}^{n+1}-w_{3}^{n+1}\right)+\Delta t^{2} K_{1} w_{2}^{n+1}}{4 m_{B}+2 \Delta t \varsigma_{B}+6 \Delta t^{2} K_{0}+2 \Delta t^{2} K_{1}}
$$

- $\quad i=2$

$$
w_{2}^{n+1}=\frac{b_{2}^{n+1}+\Delta t^{2} K_{0}\left(4 w_{1}^{n+1}+4 w_{3}^{n+1}-w_{4}^{n+1}\right)+\Delta t^{2} K_{1}\left(w_{1}^{n+1}+w_{3}^{n+1}\right)}{4 m_{B}+2 \Delta t \varsigma_{B}+6 \Delta t^{2} K_{0}+2 \Delta t^{2} K_{1}}
$$

- $\quad i=n_{\max }-1$

$$
w_{n_{x}-1}^{n+1}=\frac{b_{n_{x}-1}^{n+1}+\Delta t^{2} K_{0}\left(2 w_{n_{x}}^{n+1}+4 w_{n_{x}-2}^{n+1}-w_{n_{x}-3}^{n+1}\right)+\Delta t^{2} K_{1}\left(w_{n_{x}}^{n+1}+w_{n_{x}-2}^{n+1}\right)}{4 m_{B}+2 \Delta t \varsigma_{B}+5 \Delta t^{2} K_{0}+2 \Delta t^{2} K_{1}}
$$


- $\quad i=n_{\max }$

$w_{n_{x}}^{n+1}=\frac{b_{n_{x}}^{n+1}+\Delta t^{2} K_{0}\left(4 w_{n_{x}-1}^{n+1}-2 w_{n_{x}-2}^{n+1}\right)}{4 m_{\text {end }}+2 \Delta t \varsigma+2 \Delta t^{2} K_{0}}$

wobei

$b_{i}^{n+1}=\Delta t^{2} q_{i}^{n+1}+\left(4 m_{\text {end }}+2 \varsigma \Delta t\right) w_{i}^{n}+\left(4 m \Delta t+\varsigma \Delta t^{2}\right) \dot{w}_{i}^{n}+\Delta t^{2} m_{\text {end }} \ddot{w}_{i}^{n}$

und

$$
K_{o}=\frac{E I}{\Delta x^{4}}, K_{1} \frac{F}{\Delta x^{2}}
$$

Da es sich bei der Lösungsmatrix für die Balkenbewegung um eine Diagonalmatrix mit Hauptachsendominanz handelt, wird für die Lösung der Gleichungen (3.60) (3.64) ein iteratives Verfahren, nämlich das Gauss-Seidel-Point-By-Point-Verfahren angewendet. Für große Gleichungssysteme ist eine iterative Lösung effizienter, da weniger Rechenoperationen als bei einer direkten Lösung nötig sind [35]. 


\subsubsection{Kopplung von Strömung und Struktur}

\section{Realisierung der Gitterverschiebung}

Mit der „sliding-mesh“-Methode wird im CFD-Code die Rotation des Rührwerks aufgegeben [101]. Die Modellierung der Rührwerksschwingung wird durch Verschiebungen und Deformationen der Gitterzellen des Strömungsfeldes realisiert. Die CFX-4 spezifische Usersubroutine USRGRD für Gittermanipulationen erlaubt es, vor jedem Zeitschritt auf die Koordinaten aller Gittereckpunkte zuzugreifen und diese $\mathrm{zu}$ verändern. Dies ermöglicht die notwendigen komplexen geometrischen Änderungen des Gitternetzes.

Die Gittermanipulation erfolgt im Wesentlichen in drei übergeordneten Schritten:

1. Zurücksetzen der ursprünglichen Auslenkung

2. Verdrehen des Rührers

3. Aufsetzen der neuen Auslenkung (siehe Abbildung 3.4)

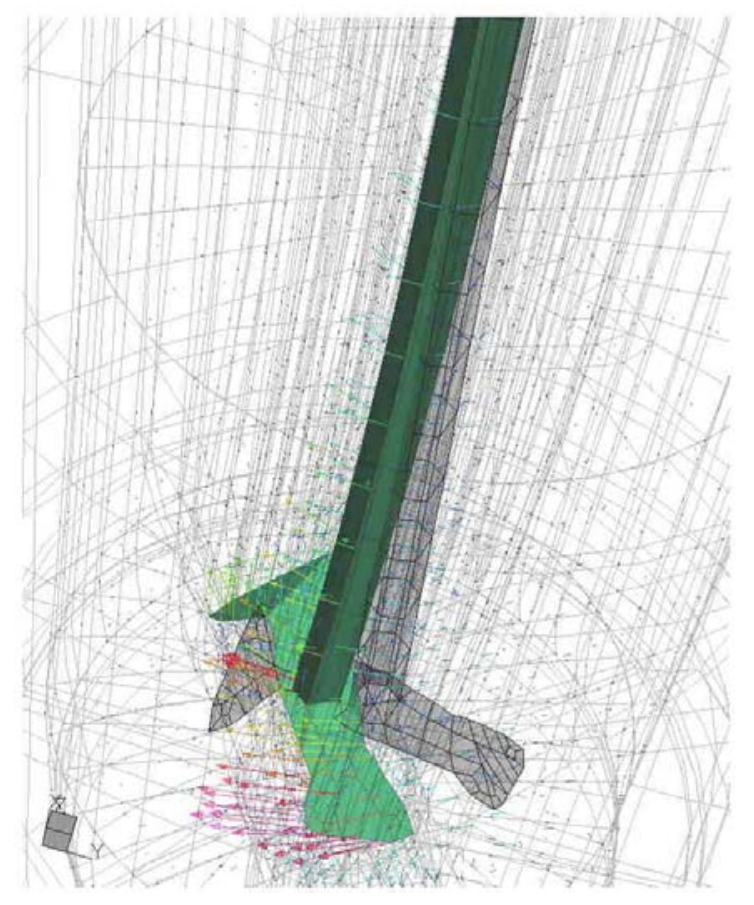

Abbildung 3.4: Drehen und Auslenken des Rührers

\section{Zurücksetzen und Verdrehen des Rührwerks}

Das Zurücksetzen und Verdrehen des Rührers in die Mittelstellung erfolgt durch Überschreiben aller Gitterkoordinaten mit den gespeicherten Koordinaten der zentrierten Stellung im letzten Zeitschritt. Dabei werden aus Gründen der Einfachheit 
auch jene Koordinaten überschrieben, die durch eine Verschiebung des Rührers nicht verändert wurden; dazu gehören zum Beispiel alle Gitterzellen im Bereich außerhalb des Stauchradius.

Die Verdrehung des Innenteils des Geometriemodells, zu dem auch das Rührwerk gehört, geschieht durch die Versetzung aller Gitterzellen des 3D-Patches um einen bestimmten Winkel um die Rotationsachse bei $y=z=0$. Der Winkel bestimmt sich dabei aus der Zeitschrittweite zwischen dem letzten und nächsten Zeitschritt und der vorgegebenen Drehzahl. Im Anschluss daran werden die aktuellen Koordinaten aller Gitterzellen für die Rücksetzung nach dem nächsten Zeitschritt gespeichert.

\section{Aufsetzen einer neuen Verschiebung im CFD-Code}

Die Auslenkung des Rührers und somit die Verschiebung angrenzender Gitterzellen folgt einem bestimmten Prinzip, bei dem das Modell zunächst in vier Höhenbereiche eingeteilt wird (siehe Abbildung 3.5). Diese sind von oben her gesehen:

- $\quad$ Bereich der freien Welle

- $\quad$ Bereich des Rührerkopfes

- Unten angrenzende gestauchte Zellen

- Gitterzellen bis zum Behälterboden, die konstant gehalten werden

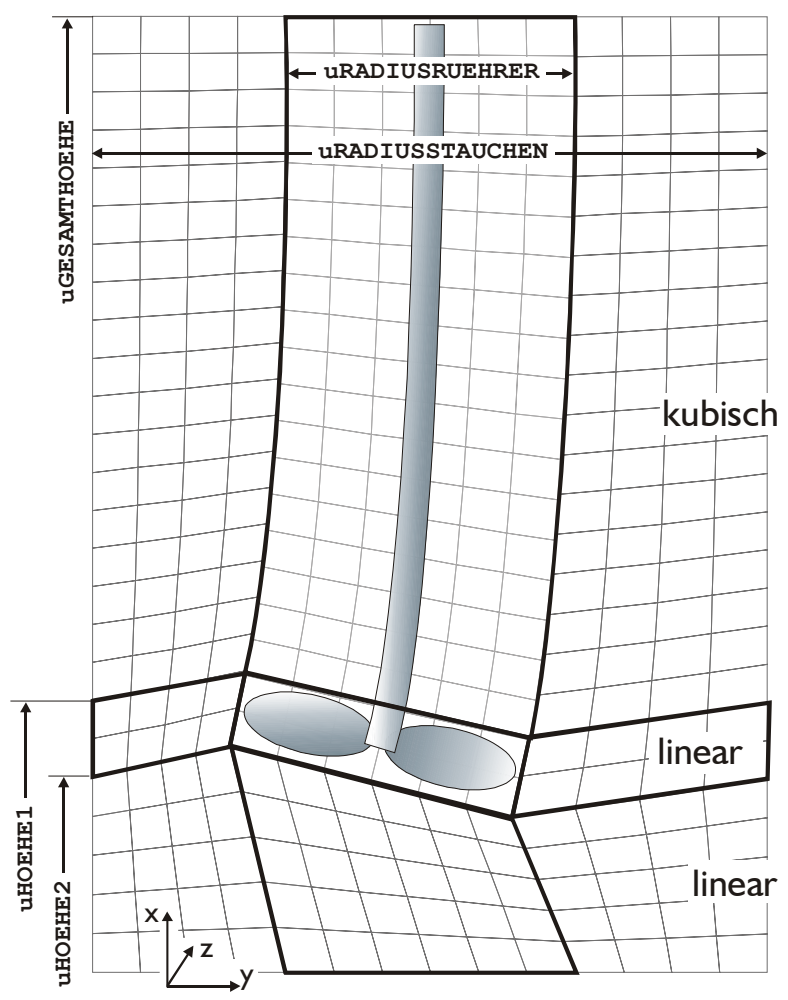

Abbildung 3.5: Modell des Rührers im ausgelenkten Gitter 
Die freie Welle wird bei dem in den CFD-Code CFX-4.4 implementierten numerischen Ersatzmodell der Rührermechanik (im Folgenden numerisches Ersatzmodell genannt) in Form einer kubischen Parabel (siehe Gleichung (3.12)) ausgelenkt, was der Biegelinie eines Balkens entspricht.

Da der Rührerkopf als starr angesehen wird, erfolgt seine Auslenkung als reine Verschiebung. Die Steigung und somit Neigung des Rührerkopfes ergibt sich aus der Ableitung der Biegelinie am unteren freien Wellenende. Die sich daraus ergebende Auslenkung wird zu dem bereits berechneten Anteil des Wellenendes addiert.

Die Gitterzellen unterhalb des Rührerkopfes werden lediglich verschoben. In einem gewissen, vorgegeben Abstand muss die Verschiebung axial abgeklungen sein, was durch einen gegen Null gehenden Faktor erreicht wird.. Das untere Ende des Bereichs (in Abbildung 3.5 nicht dargestellt) bleibt ohne Verschiebung.

Die Grenzen der einzelnen Bereiche ergeben sich aus den vorgegebenen Geometriedaten und sind unabhängig von dem für die Rotation genutzten definierten 3D-Patch-Bereich.

In radialer Richtung erfolgt eine Einteilung in drei Bereiche:

- $\quad$ direkt an den Rührer angrenzende Zellen

- daran anschließender Ring gestauchter Zellen

- $\quad$ Bereich konstant gehaltener Zellen bis zur Behälterwand

Die direkt an den Rührer angrenzenden Zellen werden bis zu einem festgelegten Radius, der größer ist als der Außenradius des Rührerkopfes, um die berechnete Auslenkung verschoben. Die Verschiebung erfolgt dabei, wie bereits erwähnt, in Form einer kubischen Parabel. Daran anschließend wird dem Gitter eine Verdrehung aufgegeben (siehe Abbildung 3.6). Der Verdrehungswinkel berechnet sich aus der Steigung der Biegelinie im Bereich des Rührerkopfes. Im Vertikalbereich des Rührerkopfes erfährt das Gitter diese Verdrehung direkt, in den oben und unten anschließenden Bereichen durch Linearfaktoren abgeschwächt. 


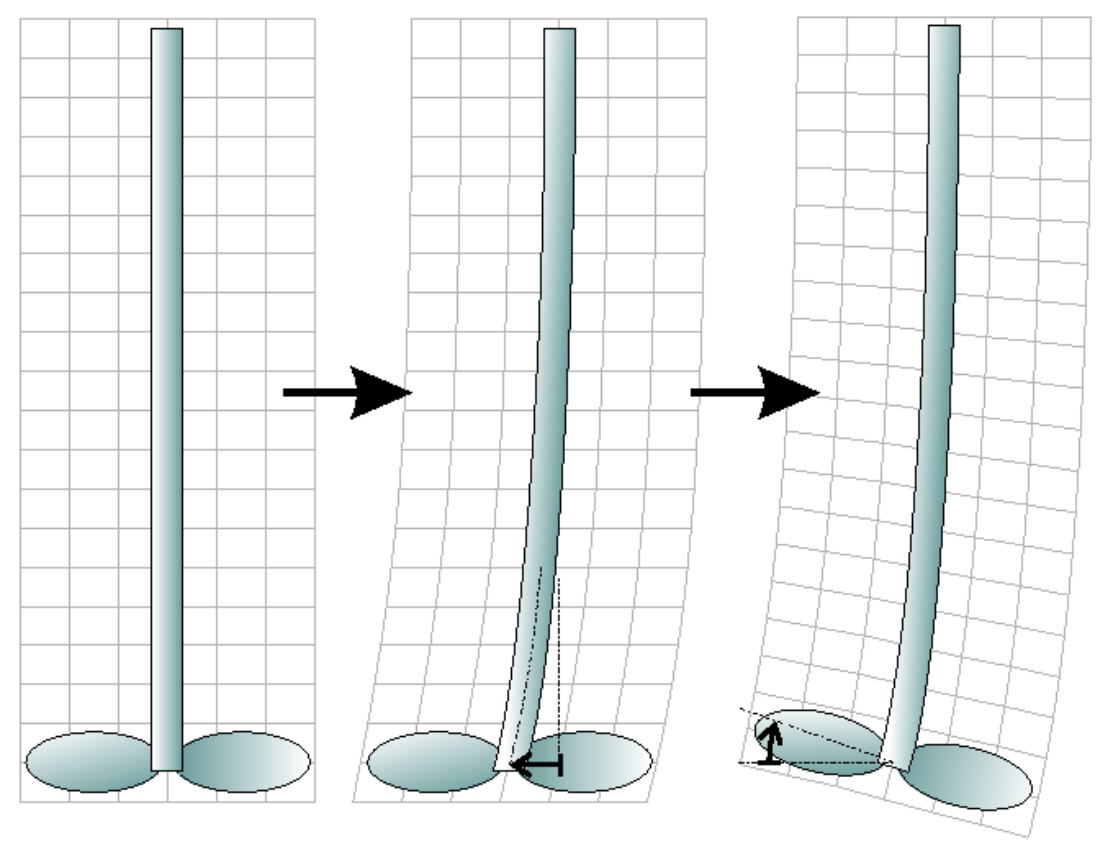

Abbildung 3.6: Teilschritte bei Aufsetzen einer Verschiebung

Um die Außenwand des Rührbehälters und eventuelle Einbauten wie Stromstörer oder dergleichen von der Auslenkung des Rührers zu entkoppeln, schließt sich an den beschriebenen inneren Teil des Gitters ein Ring von Gitterzellen an, der je nach Bedarf gestaucht beziehungsweise gedehnt wird. Im CFD-Code wird diese Methode durch Multiplikation eines Faktors an die aufgebrachte Verschiebung erzielt, der mit zunehmendem Radius nach außen hin gegen Null geht. So wird erreicht, dass sich die Verschiebung bis zum Radius uRADIUSSTAUCHEN linear abschwächt. Die gleiche Vorgehensweise wurde bereits bei den Gitterzellen angewandt, die sich nach unten hin dem Rührer anschließen. Mit einer möglichst groß gehaltenen Differenz zwischen den Radien uRADIUSRUEHRER und uRADIUSSTAUCHEN wird dabei ein sanfter Übergang und eine geringe Deformation einzelner Gitterzellen erreicht, was sich in einer besseren Konvergenz der numerischen Strömungsberechnung niederschlägt.

Im dritten, äußersten Bereich in radialer Richtung wird, wie im untersten Bereich in vertikaler Richtung, das Gitter konstant gehalten. Abbildung 3.7 zeigt die Umsetzung der Gitterverschiebung im numerischen Modell. 


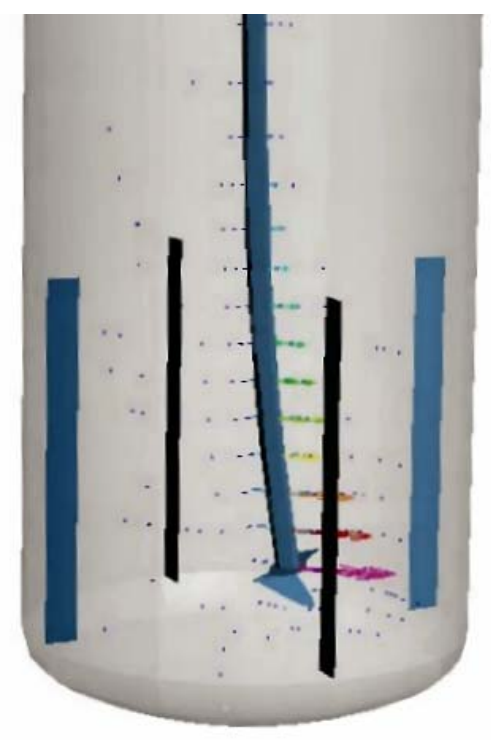

Abbildung 3.7: Auslenkung des numerischen Modells

Durch die Bewegung und Verzerrung des Gitters darf kein Kontrollvolumen, keine Masse, kein Impuls und keine Energie verloren gehen. Das sogenannte „Space Conservation Law“ (SCL), welches durch zusätzliche Gleichungen im CFD-Code CFX-4.4 erfüllt wird, trägt diesen Forderungen Rechnung [27].

\section{Auslesen der Strömungskräfte}

Für eine vollständige Fluid-Struktur-Kopplung ist es notwendig, die Reaktionen des Fluids in Form von Strömungskräften auszulesen, um mit Hilfe dieser Information die Strukturantwort zu errechnen.

Die Strömungskräfte setzen sich aus Druck- und Scherkräften zusammen. Die Druckkräfte werden durch Multiplikation des Druckes mit der Fläche berechnet. Beide Größen können im CFD-Code CFX-4.4 durch Abfrage der Wandflächen der einzelnen Gitterzellen, die an den Rührerkopf und an die Welle grenzen, bestimmt werden. Dazu lässt man den Simulationscode über alle Gitterflächen laufen, die am Rührerkopf und an der Welle liegen und liest deren Druck $p$ und Fläche $A$ aus. Der Druck ist der auf die Fläche wirkende Gesamtdruck, während die Fläche als in drei Koordinatenrichtungen projizierte Fläche vorliegt. Die gesamte Druckkraft ergibt sich aus der Summation aller Teilkräfte auf die einzelnen Gitterflächen.

Im numerischen Ersatzmodell werden die Kräfte auf die Welle dabei mit einem Faktor gewichtet, der sie als Kraft an der Endmasse des Ersatzmodells summiert, die das gleiche Moment um die Einspannung erzeugen würde (siehe Kapitel 3.2.1). Beim in 
den CFD-Code CFX-4.4 implementierten Bernoulli-Balkenmodell für die Rührermechanik (im Folgenden numerisches Balkenmodell genannt) muss über eine weitere „If'-Abfrage die Zuordnung der Fluidzellen $\mathrm{zu}$ den jeweiligen Balkenabschnitten realisiert werden.

Die folgende Abbildung stellt einen möglichen Ausschnitt des numerischen Balkenmodells dar:

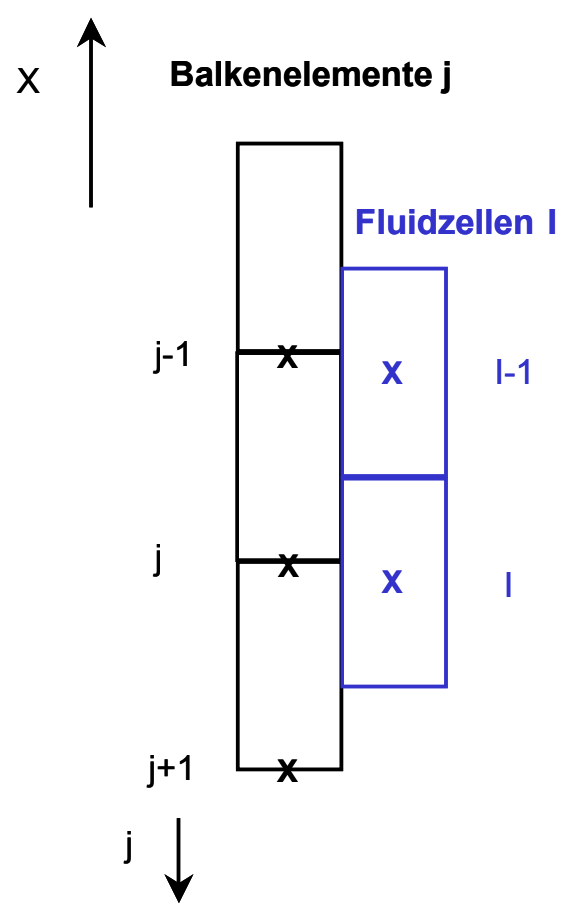

Abbildung 3.8: Zuordnung zwischen Fluid- und Balkenelementen beim numerischen Balkenmodell

Entsprechend der „If“-Bedingung wird eine Fluidzelle dem Balkenelement $j$ zugeordnet, wenn ihre Mittelpunktskoordinate in $x$-Richtung größer oder gleich der des Balkenelements und zugleich kleiner als der des darüberliegenden Balkenabschnitts $j-1$ ist.

Die Scherkräfte aus der Strömung werden ebenfalls berechnet und zu den Strömungskräften aus reiner Druckbelastung addiert.

\section{Berechnung der Auslenkung des Rührwerks}

Die Auslenkung des auf eine Einzelmasse reduzierten Rührwerks errechnet sich aus den ermittelten Druck- und Scherkräften der Strömung sowie der Unwuchtkraft des Rührers. Dazu werden die bestimmten Kräfte in ihren Koordinatenrichtungen addiert 
und in die zeitlich diskretisierte Differentialgleichung des Einmassenschwingers eingesetzt. Je nach gewählter Diskretisierung ist das die Gleichung (3.32) für das BDF-Verfahren, Gleichung (3.35) für das Leapfrog-Verfahren, Gleichung (3.40) für das Crank-Nicholson-Verfahren und Gleichung (3.44) für das Verfahren nach Newmark.

Die jeweils benötigte Kraft $F^{n+1}$ zum neuen Zeitschritt wird dabei näherungsweise durch die Kraft $F^{n}=F_{\text {Druck }}+F_{\text {Scher }}+F_{\text {Unwucht }}$ ersetzt.

\section{Aufgabe von Wandgeschwindigkeiten}

Zur korrekten Berechnung der Strömung werden die Geschwindigkeiten der bewegten Wände benötigt, an denen die Haftbedingung gilt. Dazu gehören die Berandungen der Welle und der Rührerblätter. Im CFD-Code CFX-4.4 werden die Randbedingungen der Strömung an den Wänden mit Hilfe der Vektoren $A, B$ und $C$ beschrieben, die über folgende Gleichung definiert sind [101]:

$$
A_{i} \cdot \dot{w}_{i}+B_{i} \cdot \tau_{i}=C_{i}
$$

Dabei beschreibt $\tau$ die Scherspannung an der Wand.

Im vorliegenden Fall soll die Geschwindigkeit festgelegt und die Scherspannung frei gelassen werden. Dies wird durch Setzen von

$$
A=\left(\begin{array}{l}
1 \\
2 \\
3
\end{array}\right) ; B=\left(\begin{array}{l}
0 \\
0 \\
0
\end{array}\right) ; C=\left(\begin{array}{l}
\dot{w}_{x} \\
\dot{w}_{y} \\
\dot{w}_{z}
\end{array}\right)
$$

erreicht.

Im Simulations-Code CFX-4.4 geschieht dies durch Überlaufen aller Wandpunkte und Aufgabe der entsprechend der Position umgerechneten Geschwindigkeiten, die zuvor mit den Auslenkungen berechnet wurden.

\section{Festlegung der Zeitschrittweite}

Die Zeitschrittweite ist bei der vorliegenden Fluid-Struktur-Kopplung nicht nur für die Konvergenz der Strömungsberechnung sondern zusätzlich durch die Strukturreaktion in ihrer Größe begrenzt.

Im CFD-Code CFX-4.4 ist es nicht möglich, innerhalb eines Zeitschritts die voneinander abhängigen Größen „F“ und „ $w^{\prime \prime}$ iterativ zu bestimmen. Das Programm fordert eine unveränderliche Modellstruktur als Randbedingung jeden Zeitschritts. Es 
muss daher bei allen Zeitdiskretisierungsmethoden zur Berechnung der Auslenkung $w^{n+1}$ zum neuen Zeitschritt $t^{n+1}$ die Kraft $F^{n}$ aus dem vorherigen Zeitschritt $t^{n}$ verwendet werden. Durch die Wahl einer geringen Zeitschrittweite wird der dadurch auftretende Diskretisierungsfehler bei den impliziten Verfahren möglichst gering gehalten.

\subsubsection{Testrechnungen und Validierung des numerischen Ersatzmodells}

\section{Testrechnungen im Leerlauf}

Bei der numerischen Simulation lassen sich verschiedene Parameter variieren, die Einfluss auf die Genauigkeit der Berechnungen, das Konvergenzverhalten und auch auf die Rechenzeit haben.

Für die ersten Testrechnungen des numerischen Ersatzmodells im Leerlauf werden zwei entscheidende Parameter untersucht:

Zum einen wird betrachtet, wie sich die Anzahl der Umdrehungen des Rührers auf die Auslenkungsamplituden (siehe Gleichung (3.68)) auswirkt. Zum anderen wird die Anzahl der Zeitschritte pro Umdrehung untersucht. Eine Zusammenfassung der erhaltenen Ergebnisse liefert Abbildung 3.11.

Beispielhaft werden für die folgenden Testrechnungen die Ergebnisse eines Schrägblattrührers dargestellt. Das Gitternetz des im CFD-Code modellierten Rührbehälters mit Schrägblattrührer ist in Abbildung 3.9 zu sehen. Abbildung 3.10 zeigt das gemittelte Geschwindigkeitsfeld des modellierten Schrägblattrührer.

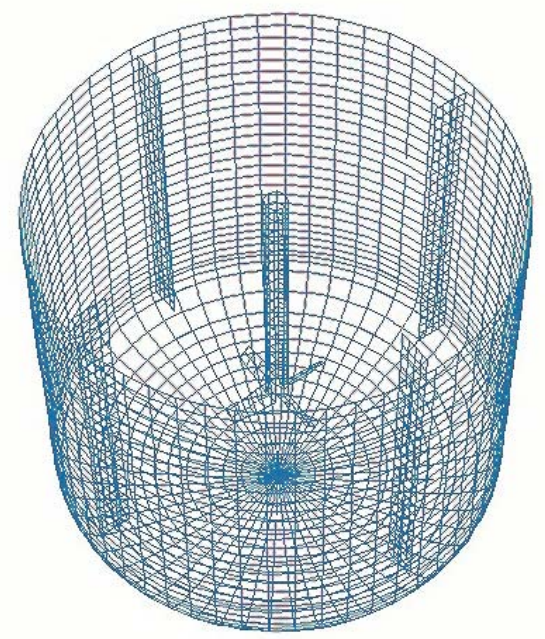

Abbildung 3.9: Oberflächengitternetz des Schrägblattrührers

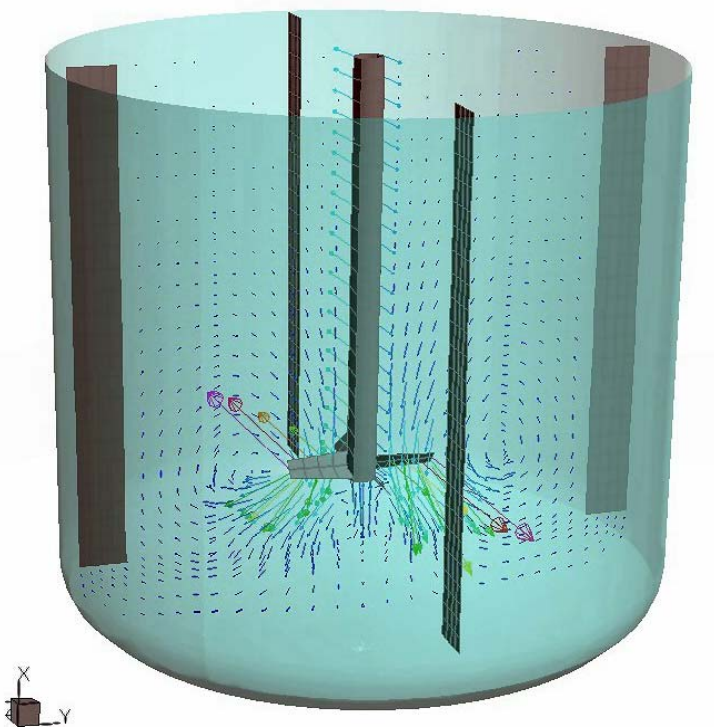

Abbildung 3.10: Geschwindigkeitsfeld des Schrägblattrührers 
Zur Auswertung des Frequenzverhaltens des numerischen Ersatz- und des Balkenmodells werden Zeitreihen der Verschiebungen mit RMS-Analyse untersucht und über der Drehzahl aufgetragen.

Der RMS-Wert einer Reihe aus s Elementen $w_{j}$ berechnet sich aus der Gleichung:

$$
w^{R M S}=\sqrt{\frac{1}{s} \sum_{j=1}^{s}\left(w_{j}-\bar{w}\right)^{2}}
$$

mit dem arithmetischen Mittelwert

$$
\bar{w}=\frac{1}{s} \sum_{j=1}^{s} w_{j} .
$$

Die RMS-Amplitude ist sozusagen der gemittelte Radius der Rührerauslenkung und kann an jeder beliebigen Stelle der Welle bestimmt werden.

Es ist darauf zu achten, nur Werte, ab denen der Rührer quasistationär eingelaufen ist, einzubeziehen. Anlaufvorgänge würden das Ergebnis verfälschen.

\section{Anzahl der Umdrehungen}

Die optimale Ausnutzung der Rechenkapazitäten verlangt, dass die durchgeführten numerischen Berechnungen so kurz wie möglich gehalten werden. Die Dauer einer Berechnung hängt somit entscheidend von der Anzahl der Umdrehungen, die der Rührer bei der Simulation durchlaufen soll, ab.

Um aussagekräftige und zuverlässige Ergebnisse zu erhalten, werden zur Berechnung der RMS-Amplitude die letzten 10 Rührerumdrehungen eines Simulationslaufes verwendet. Voraussetzung dafür ist, dass das Rührwerk bereits eingeschwungen und der Anlaufvorgang keine Rolle mehr spielt. Es ist festzustellen, dass sich ein Unterschied bei den RMS-Amplituden zwischen 20 Umdrehungen und 40 Umdrehungen, vor allem im Bereich der kritischen Drehzahl, ausbildet (siehe Abbildung 3.11). Eine Erklärung hierfür bieten die Abbildungen 3.12 und 3.13. 


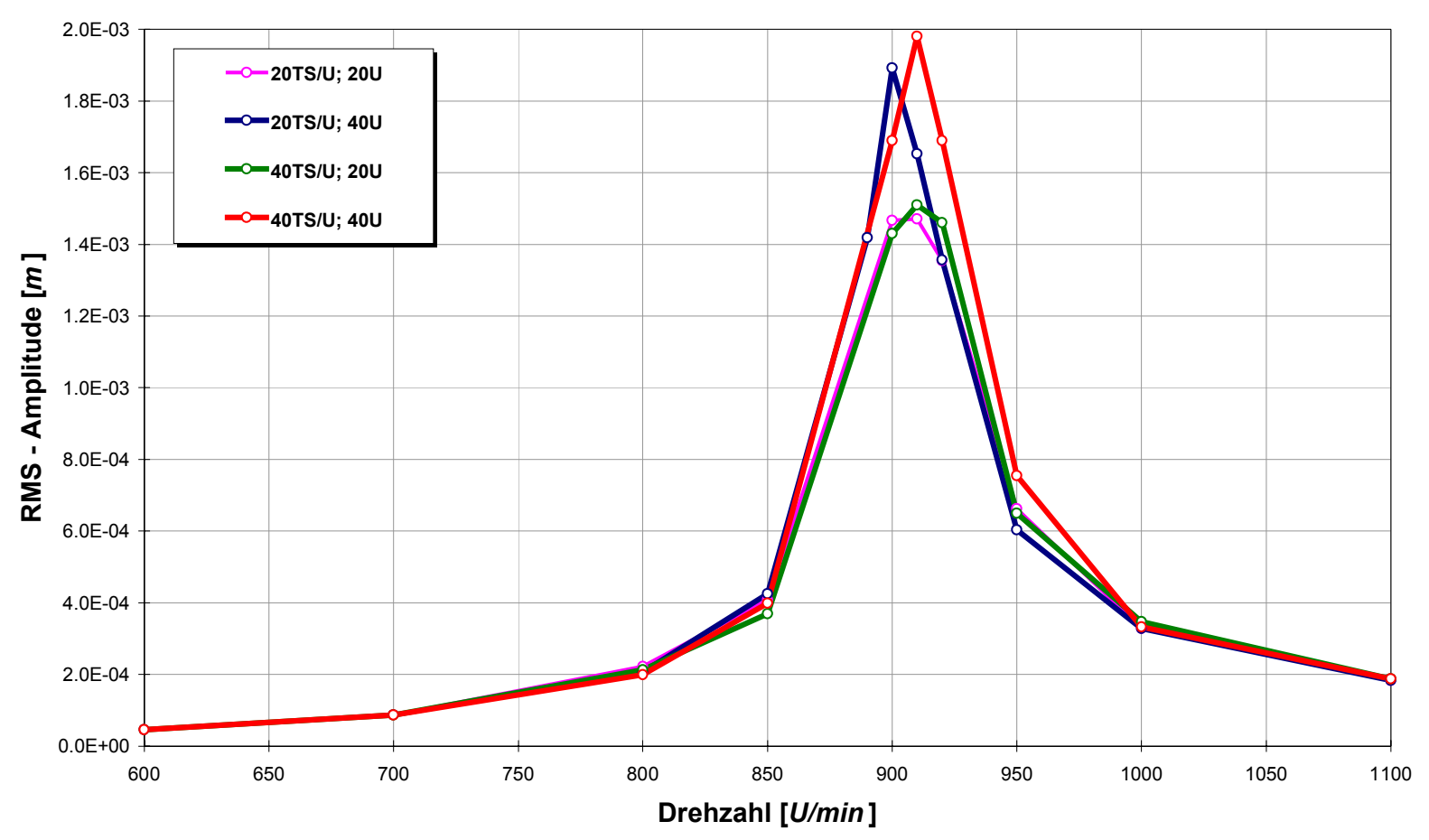

Abbildung 3.11: RMS-Amplituden im Leerlauf bei verschiedenen Zeitschrittweiten und unterschiedlicher Anzahl von Umdrehungen

Abbildung 3.12 zeigt, dass sich das Rührwerk im unterkritischen Drehzahlbereich ab etwa 15 Umdrehungen (600TS) einschwingt. Der Überschwinger zu Beginn der Simulation rührt vom sofortigen Anfahrvorgang auf Betriebsdrehzahl her und klingt langsam ab. Er tritt nur bei höheren Drehzahlen auf, jedoch nicht bei der kritischen Drehzahl. Hier steigt die Auslenkung stetig mit der Zeit an (siehe Abbildung 3.13). 


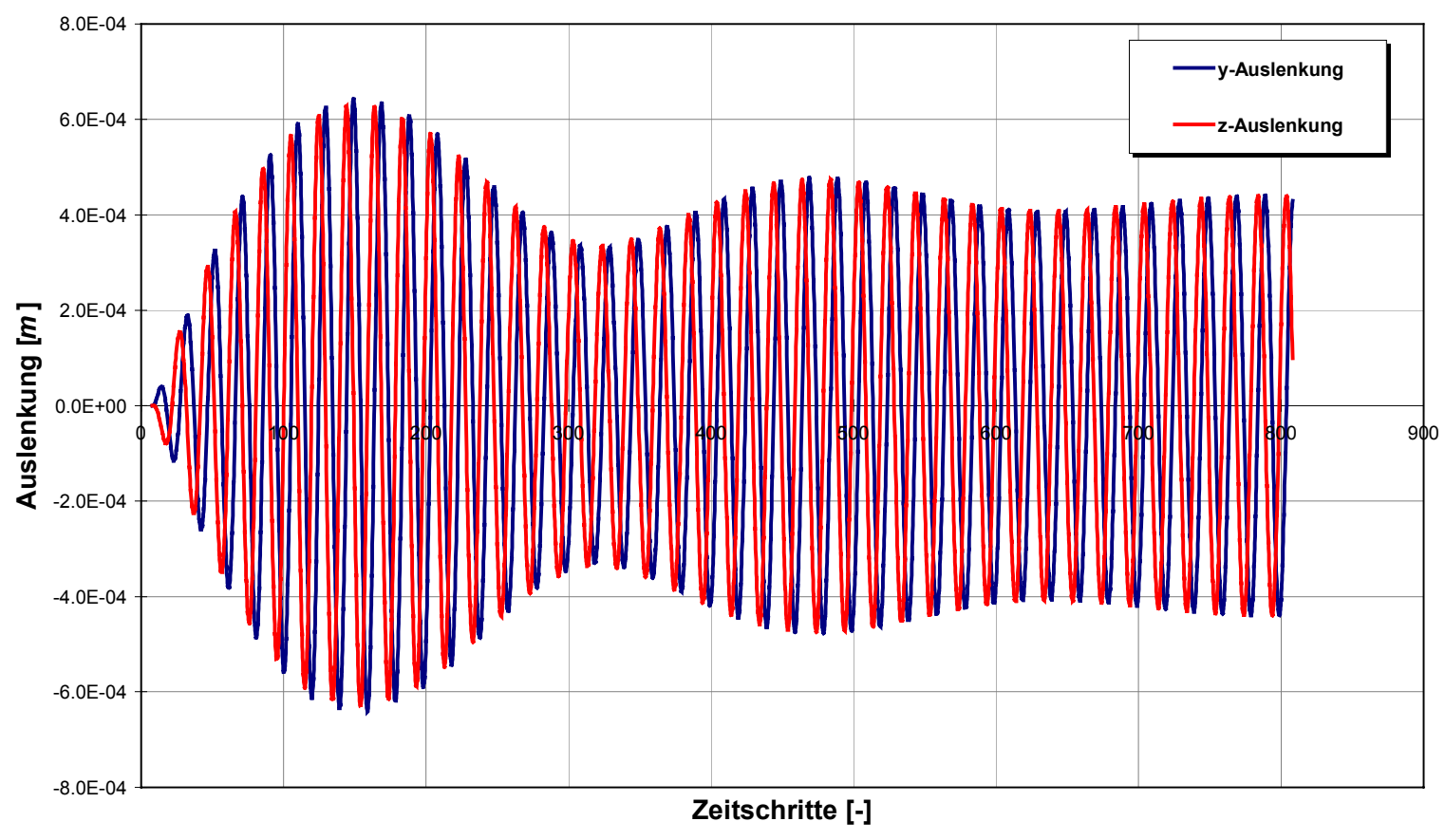

Abbildung 3.12: Auslenkung des Rührwerks bei $n<n_{k r i t}$

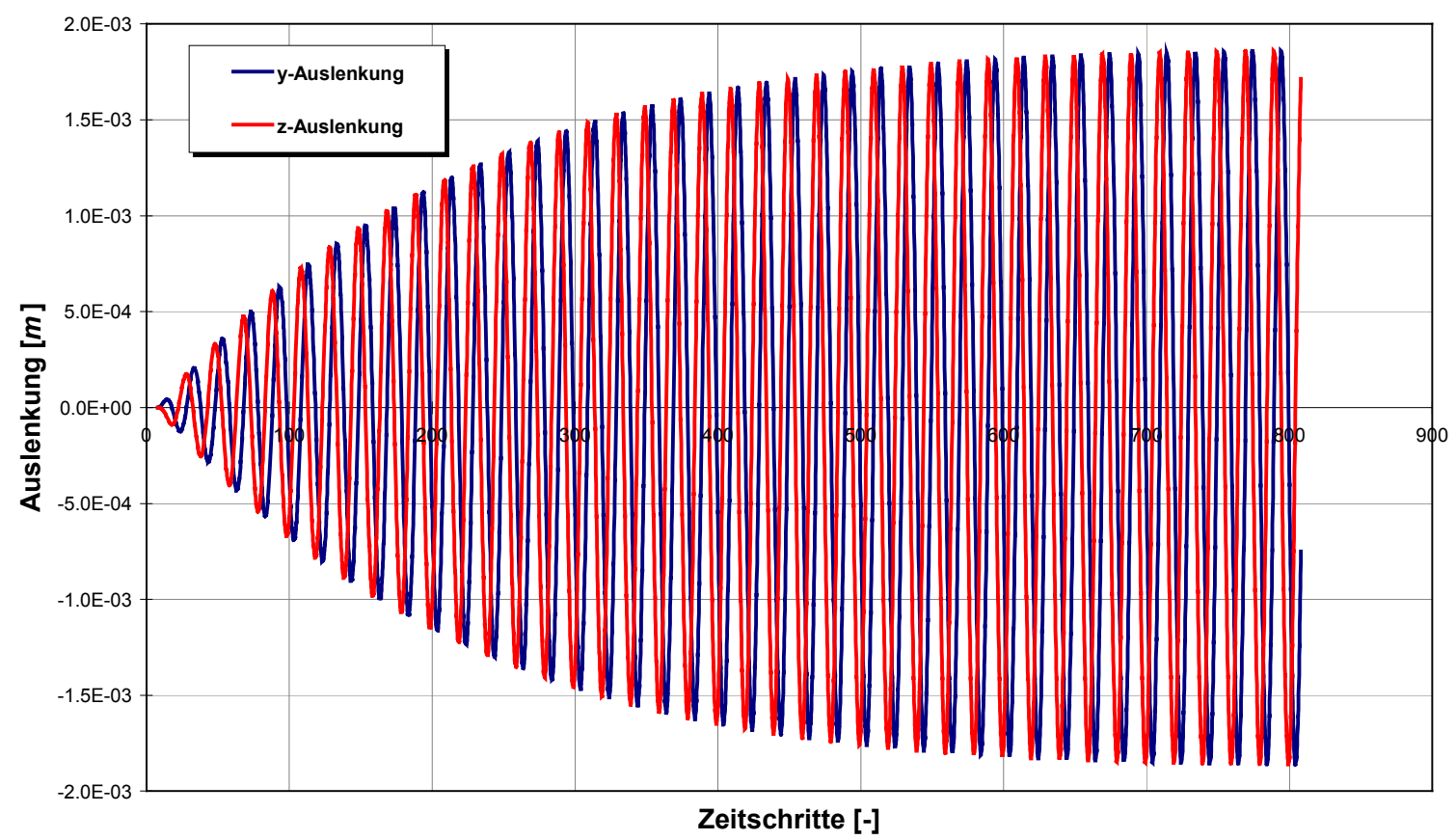

Abbildung 3.13: Auslenkung des Rührwerks bei $n=n_{\text {krit }}$

\section{Zeitschritte pro Umdrehung $(T S / U)$}

Die Auswirkung der Zeitschrittweite auf die RMS-Amplitude und die kritische Drehzahl kann wiederum anhand Abbildung 3.11 erkannt werden. 
Die kritische Drehzahl liegt bei einer Zeitschrittweite von $40 T S / U$ bei $910 U / \mathrm{min}$. Sie verringert sich bei größerer Zeitschrittweite $(20 T S / U)$ geringfügig auf $900 U / m i n$. Hier ist also ein Tendenz zu kleineren kritischen Drehzahlen bei Vergrößerung der Zeitschrittweite erkennbar.

\section{Validierung des numerischen Ersatzmodells im Leerlauf}

Wie bereits in Kapitel 1.3 erwähnt, existieren für die Berechnung der kritischen Drehzahl im Leerlauf zuverlässige analytische Berechnungsformeln, welche für eine Validierung des Ersatzmodells verwendet werden können.

Mit Gleichung (1.13) ergibt sich die erste Eigenfrequenz $f_{l}$ bzw. die kritische Drehzahl $n_{\text {krit }}$ eines Schrägblattrührers zu 911U/min.

Mit dem Finite-Element Softwarepaket Marc/Mentat 7.3 wurde der Schrägblattrührer ebenfalls nachmodelliert und mittels Modalanalyse ein Wert von 909U/min ermittelt. Das FE-Verfahren kann im Rahmen der Modellierungsmöglichkeiten im Leerlauf als das genaueste Ergebnis betrachtet werden.
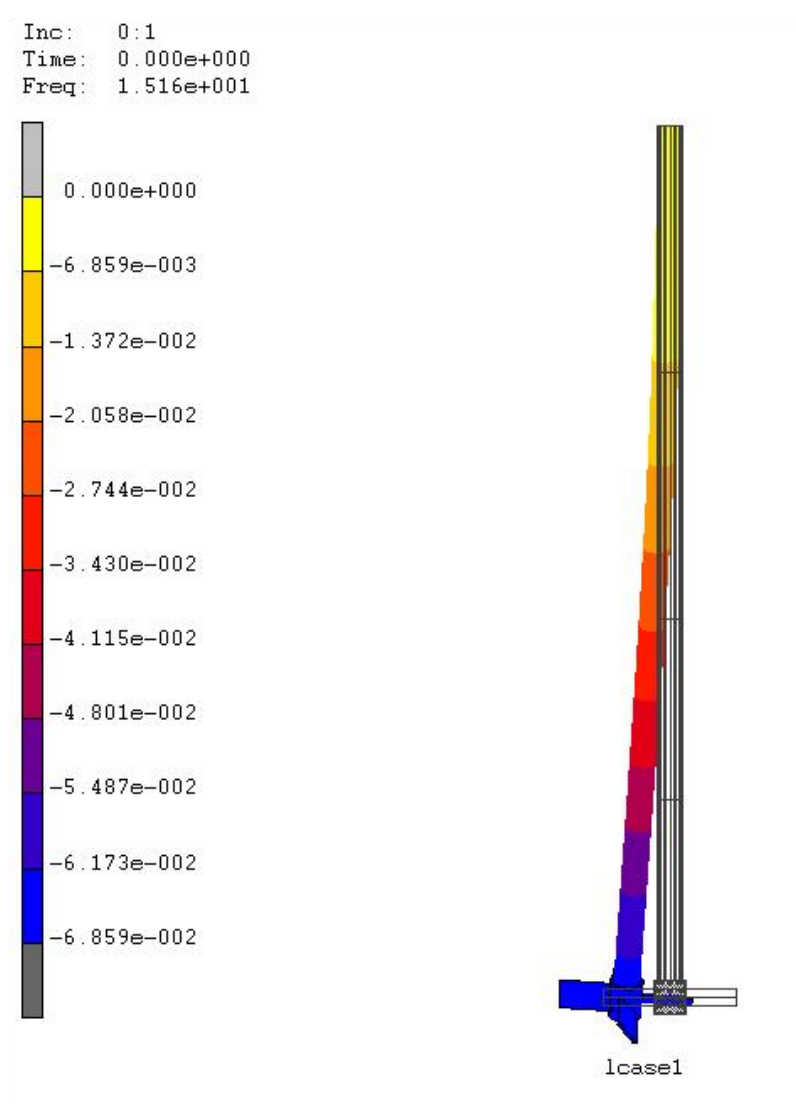

Msc $\mathbf{X}$

Displacement X

Abbildung 3.14: FEM-Modell des untersuchten Schrägblattrührers 
Zum Vergleich des numerischen Ersatzmodells mit dem numerischen Balkenmodell werden die RMS-Amplituden des Rührers über der Drehzahl aufgetragen. Nach umfassenden Parameterstudien mit dem numerischen Balkenmodell im Leerlauf wurde die Anzahl der Diskretisierungselemente auf 7 und die Zeitschrittweite auf 180TS/U gesetzt, um stabile Simulationsläufe zu erhalten.

Wie aus Abbildung 3.15 zu erkennen ist, liegen bei beiden Modellen die berechneten kritischen Drehzahlen übereinstimmend bei ca. 910U/min.

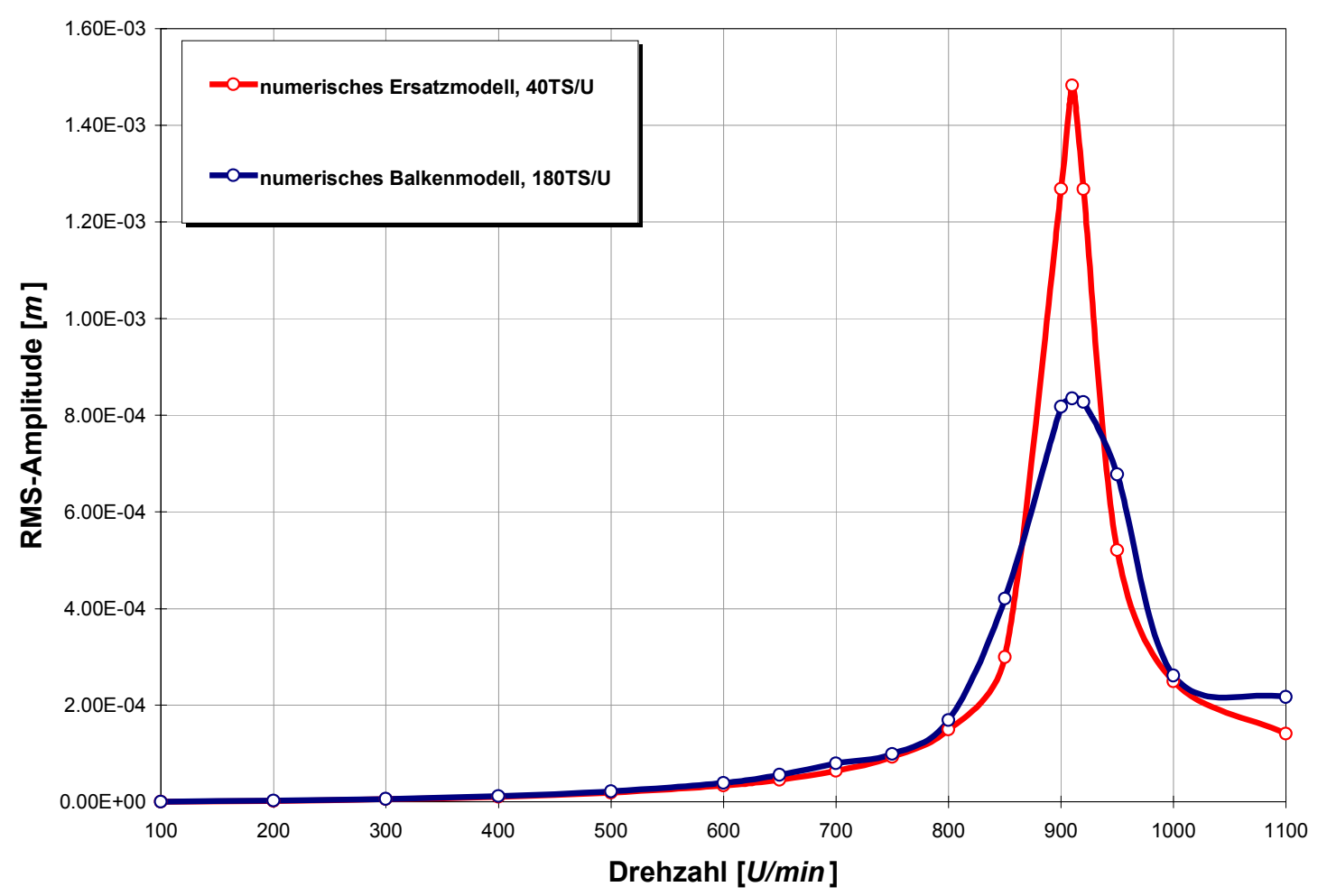

Abbildung 3.15: Vergleich numerisches Ersatzmodell mit numerischem Balkenmodell

In Tabelle 3.2 sind die ermittelten kritischen Drehzahlen der unterschiedlichen Berechnungsmethoden nochmals zusammenfassend dargestellt.

\begin{tabular}{||c|c||}
\hline Methode & $\mathbf{n}_{\text {krit }}(\boldsymbol{U} / \mathbf{m i n})$ \\
\hline Numerisches Ersatzmodell & 910 \\
\hline Numerisches Balkenmodell & 913 \\
\hline Analytische Lösung & 911 \\
\hline FEM & 909 \\
\hline
\end{tabular}

Tabelle 3.2: Vergleich der kritischen Drehzahlen 
Es zeigt sich bezüglich der kritischen Drehzahl eine sehr gute Übereinstimmung zwischen dem numerischen Ersatzmodell und den Validierungslösungen. Auftretende physikalische Effekte werden somit alle richtig erfasst. Das numerische Ersatzmodell des Rührwerks kann somit hinsichtlich des Frequenzverhaltens als validiert betrachtet werden.

Zur zusätzlichen Kontrolle des Frequenzverhaltens und zur Überprüfung der Schwingungsamplituden wird das numerische Ersatzmodell in Kapitel 5 zusätzlich mit experimentellen Untersuchungen verglichen.

\section{Parameterstudien im Fluid (Wasser)}

In diesem Abschnitt werden die folgenden numerischen Parameter und deren Einfluss auf das numerische Ersatzmodell in Wasser überprüft:

- Gitterfeinheit des Strömungsfelds

- Turbulenzmodell

- Iterationszahl

- Zeitschrittweite

- Zeitliches Diskretisierungsverfahren der Struktur

Mit den gefundenen numerischen Parametern wird das numerische Ersatzmodell zunächst mit dem numerischen Balkenmodell und später in Kapitel 5 mit experimentellen Ergebnissen validiert.

Durch die äußere Dämpfung des Fluids läuft sich das Rührwerk über den gesamten Drehzahlbereich schneller auf eine quasistationäre Kreisbahn ein als beim Betrieb im Leerlauf. Zur Bildung von aussagekräftigen RMS-Amplitudenwerten ist es deshalb ausreichend, 20 Umdrehungen zu simulieren [14].

\section{Anzahl der Gitterzellen und Turbulenzmodellierung}

Da die Anzahl der verwendeten Gitterzellen des numerischen Modells eng mit dem verwendeten Turbulenzmodell zusammenhängt, werden diese beiden numerischen Parameter geschlossen in Abbildung 3.16 präsentiert. 


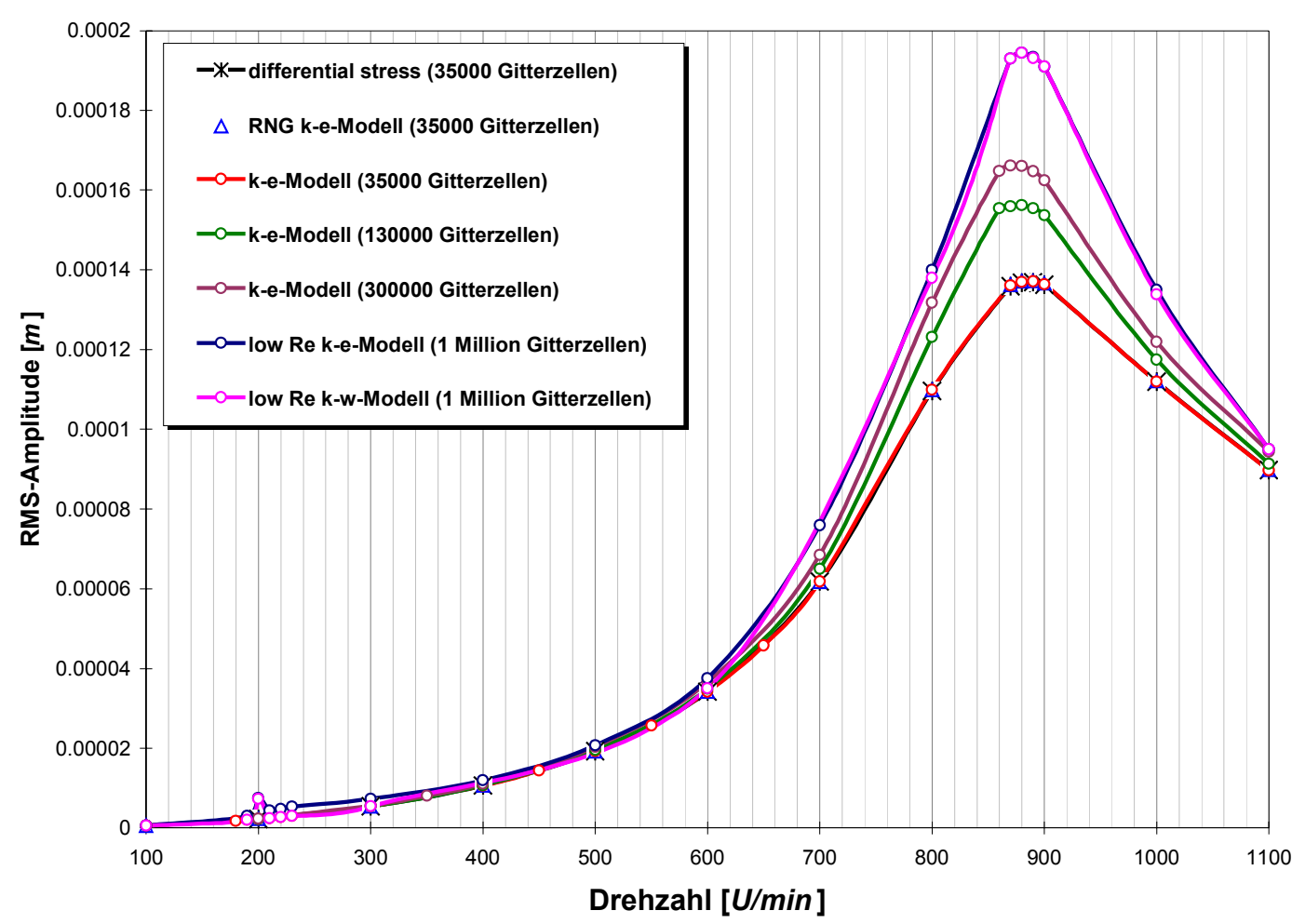

Abbildung 3.16: Vergleich verschiedener Turbulenzmodelle und Gitterfeinheiten

Die RMS-Auslenkungen des Rührwerks nehmen beim $k-\varepsilon$ Turbulenzmodell mit steigender Gitteranzahl zu, was darauf zurückzuführen ist, dass die resultierenden Strömungskräfte auf das gesamte Rührwerk in Axial-, Radial- und Tangentialrichtung mit größerer Gitteranzahl geringer werden.

Die Strömungskräfte auf die einzelnen Rührerblätter sind beim feineren Gitter größer, da die turbulente kinetische Energie auch zunimmt. Jedoch kommt es durch die feinere Gitterzellenauflösung $\mathrm{zu}$ einer exakteren geometrischen Auflösung der Strömungskräfte, was sich dementsprechend im Ergebnis niederschlägt.

Auffällig ist weiterhin, dass das RNG- $k-\varepsilon$ Modell, das differential-stress Modell und das $k-\varepsilon$ Turbulenzmodell bei einem Berechnungsgitter von 35000 Gitterzellen keinerlei Unterschiede aufweisen.

Die beiden Low-Re Turbulenzmodelle, die bis zur Wand durchintegrieren zeigen in ihrer Tendenz größere Einzelströmungskräfte auf die Rührerblätter. Die Strömungskraftresultierenden in die drei Koordinatenrichtungen sind jedoch geringer als bei den Turbulenzmodellen mit Wandfunktionen, womit sich die größeren RMSAmplituden erklären lassen.

Zusammenfassend ergibt sich, dass die beiden Low-Re-Modelle aufgrund des enormen Rechenzeit- und Speicherplatzbedarfs als unpraktikabel zu bezeichnen sind. Der 
Einfluss des Turbulenzmodells und der Gitterfeinheit auf die RMS-Amplitude des Rührwerks ist im gesamten Drehzahlbereich absolut als gering zu werten, was aus Abbildung 3.16 auch deutlich erkennbar ist.

\section{Iterationszahl pro Zeitschritt}

Aufgrund der Auslenkung des Rührwerks ergeben sich sinusläufige Verläufe der aufsummierten Fluidkräfte auf das Rührwerk in $y$ - und $z$-Richtung. In axialer Richtung ergibt sich entsprechend der Förderwirkung eine Gegenkraft des Fluids.

Abbildung 3.17 zeigt eine deutliche Abhängigkeit der Genauigkeit von der Anzahl der Iterationen pro Zeitschritt. Erst ab etwa 10 Iterationen pro Zeitschritt lassen sich realistische Strömungskräfte herauslesen.

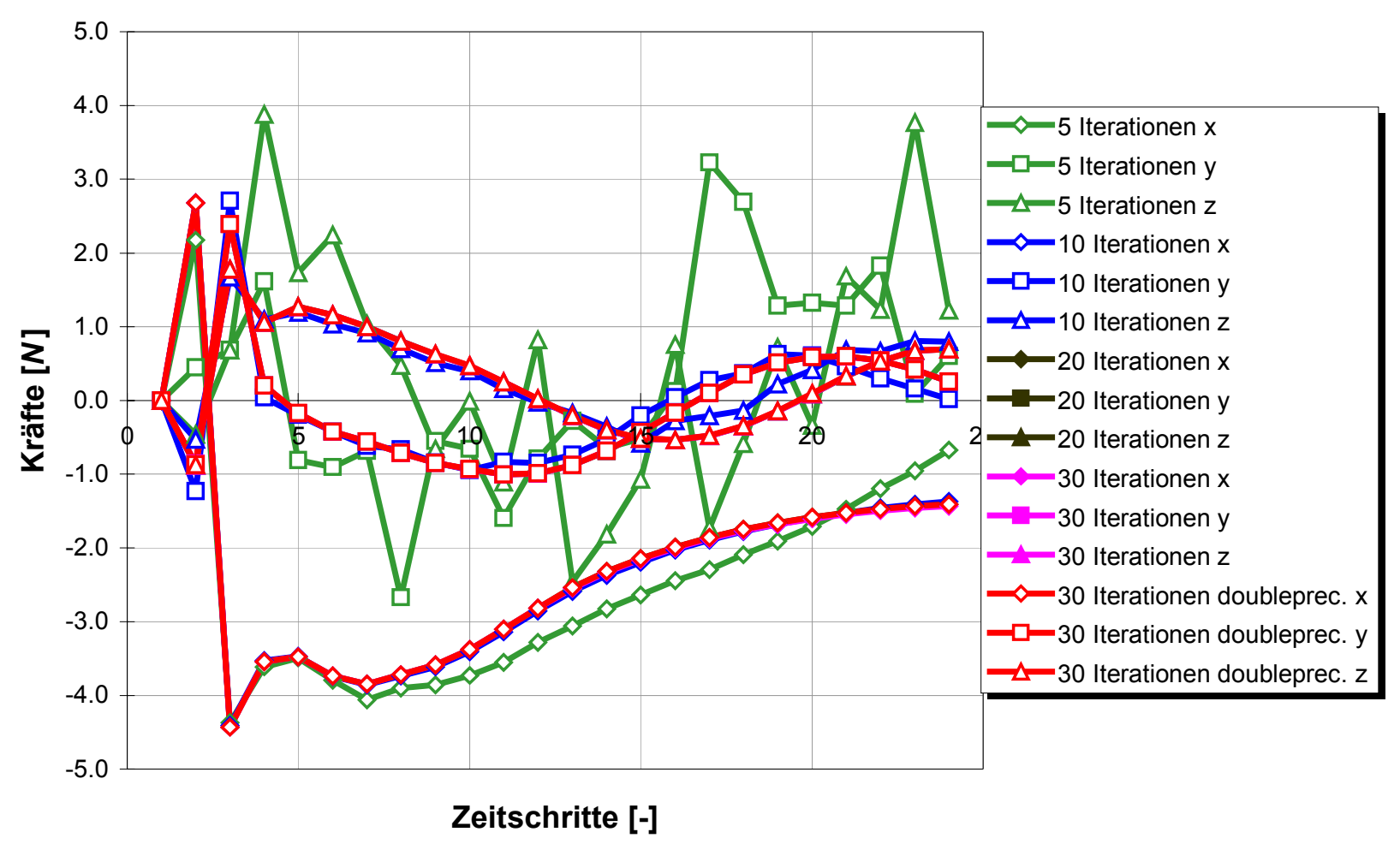

Abbildung 3.17: Ermittelte Kräfte in Abhängigkeit von der Iterationszahl

\section{Zeitliche Diskretisierung}

Die Abhängigkeit der RMS-Amplitude des Rührwerks vom zeitlichen Diskretisierungsverfahren der Struktur und der damit verbundenen Zeitschrittweite wird in Abbildung 3.18 dargestellt. Ergänzend muss erwähnt werden, dass diese Berechnungen für den Schrägblattrührer mit einer vorab geschätzten Exzentrizität $\varepsilon$ von 2,0E-04 angestellt wurden. 
Als Referenz für die Beurteilung der Genauigkeit wird das Newmark-Verfahren mit der geringsten Zeitschrittweite von 360TS/U herangezogen, da dieses Verfahren schon häufig mit guten Resultaten für dynamische Schwingungen verwendet wurde und frei von numerischer Dämpfung ist [35].

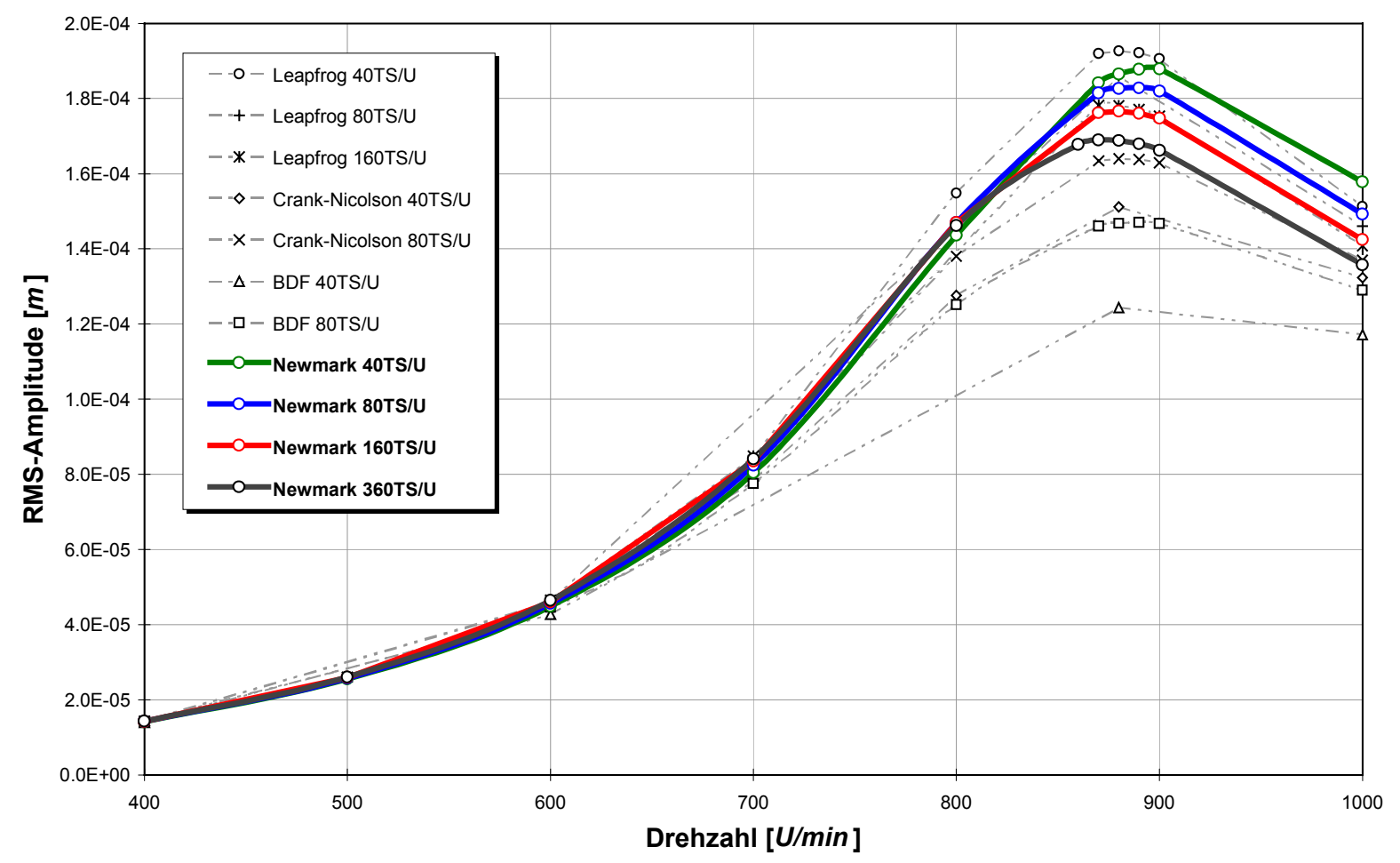

Abbildung 3.18: Vergleich der zeitlichen Diskretisierungsansätze bei unterschiedlichen Zeitschrittweiten

Ein Vergleich der Diskretisierungsansätze zeigt, dass vor allem die impliziten Verfahren (BDF und Crank-Nicolson) bei großer Zeitschrittweite zu geringe RMSWerte wiedergeben. Das schlechte Abschneiden des BDF-Verfahrens ist damit zu begründen, dass es sich um ein Verfahren mit einem Abbruchfehler 1. Ordnung in der Zeit handelt, wodurch die Genauigkeit leidet.

Das Crank-Nicolson-Verfahren ist von 2. Ordnung und ist deshalb genauer als das BDF-Verfahren. Es bildet die einzusetzenden Strömungskräfte zur Hälfte aus $F^{n}$ und zur Hälfte aus $F^{n+1}$. Der auftretende Diskretisierungsfehler im numerischen Ersatzmodell, da $F^{n}$ anstelle von $F^{n+1}$ verwendet werden muss, spielt daher eine kleinere Rolle als beim BDF-Verfahren. Eine Verringerung der Zeitschrittweite hat auch hier ein besseres Ergebnis zur Folge. 
Das explizite Leap-Frog-Verfahren liefert sehr geringe Abweichungen vom NewmarkVerfahren, da hierbei nicht die Kraft $F^{n+1}$ durch $F^{n}$ ersetzt werden muss. Es wird herleitungsgemäß $F^{n}$ zur Berechnung von $w^{n+1}$ verwendet. Die RMS-Amplituden dieses Verfahrens liegen bei 40TS/U etwas über den Werten des Newmark-Verfahrens. Eine Absenkung der Zeitschrittweite auf 80TS/U bzw. 160TS/U bewirkt jedoch eine Annäherung im Hinblick auf die RMS-Amplitude.

Ein Vergleich des Newmark-Verfahrens für unterschiedliche Zeitschrittweiten spiegelt auch den Zusammenhang zwischen der Anzahl der Zeitschritte pro Umdrehung und der kritischen Drehzahl wider. Sie reduziert sich von 900U/min beim NewmarkVerfahren mit 40TS/U auf 870U/min beim Newmark-Verfahren mit 360TS/U.

\section{Vergleich des numerischen Ersatzmodells mit dem numerischen Balkenmodell}

Für das numerische Balkenmodell kann nach umfangreichen Parameterstudien die Aussage getroffen werden, dass mindestens 1000TS pro Umdrehung und, wie im Leerlauf, mindestens 7 Balkendiskretisierungselemente für die Rührerwelle nötig sind, um sinnvolle und vor allem stabile Simulationsergebnisse in Fluiden zu erhalten.

Die Simulationsergebnisse des numerischen Balkenmodells unterscheiden sich, bezüglich kritischer Drehzahl und Auslenkungsamplitude, letztendlich kaum von denen des numerischen Ersatzmodells (siehe Abbildung 3.19).

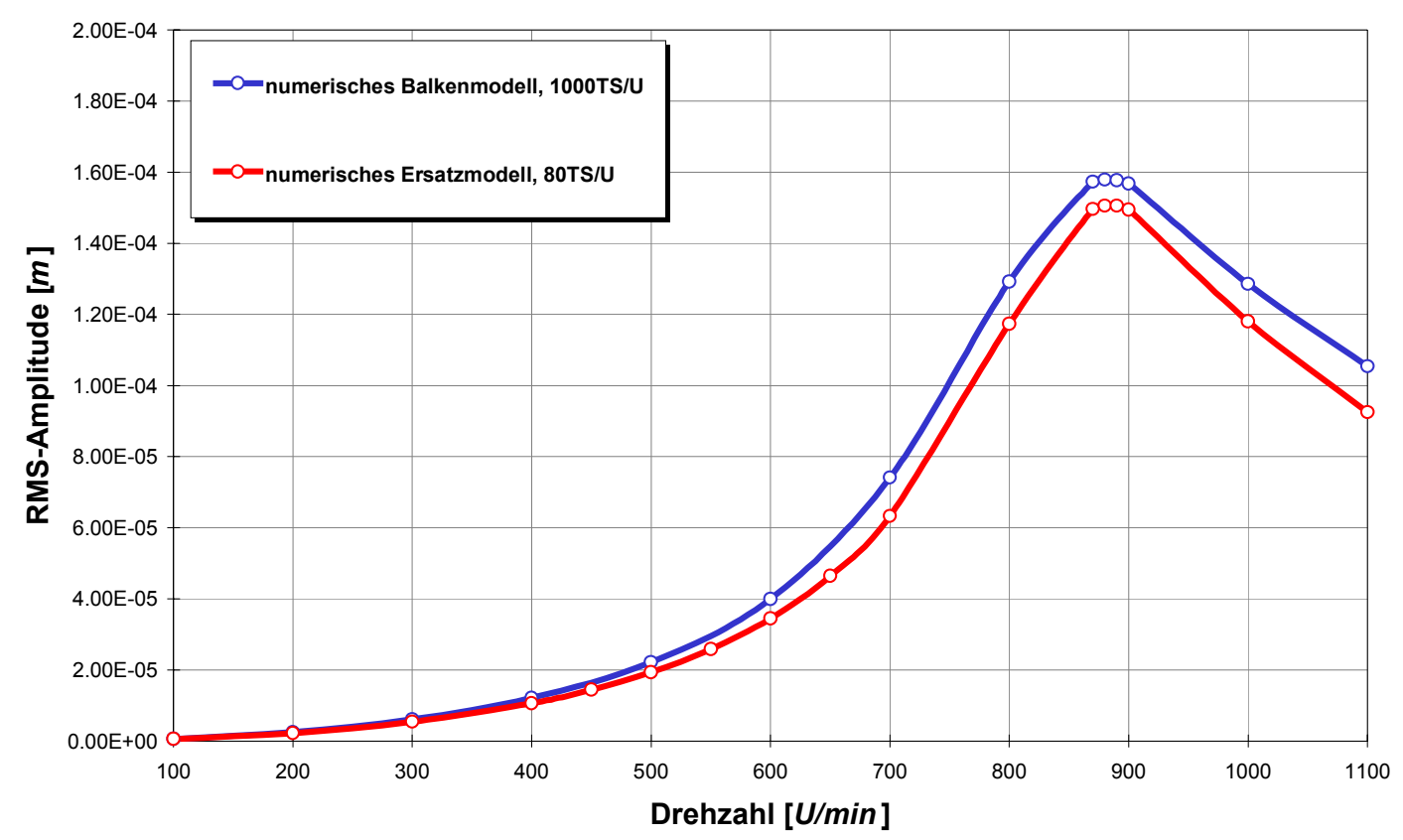

Abbildung 3.19: Vergleich numerisches Ersatzmodell-Balkenmodell in Wasser 
Das numerische Balkenmodell ist somit bei gleicher Rechengenauigkeit insgesamt wesentlich rechenzeitaufwendiger und vor allem instabiler als das numerische Ersatzmodell.

Zur Validierung der gefundenen numerischen Parameter und zur Überprüfung des numerischen Ersatzmodells im Fluid werden zusätzlich noch Experimente durchgeführt, da hierzu keine verlässlichen analytischen Berechnungen möglich sind. Ein Vergleich zwischen den Simulations- und den experimentellen Ergebnissen wird in Kapitel 5 dargestellt.

\subsubsection{Berücksichtigung der Einspannsteifigkeit des Rührwerks}

In den statischen analytischen Berechnungsmodellen (siehe Kapitel 2.6) werden die Annahmen getroffen, dass das Rührwerk an einem unendlich steifen System befestigt ist, was nicht unbedingt der Realität entspricht. Im Rahmen dieser Arbeit werden drei unterschiedliche Einspanngeometrien modelliert und untersucht:

- $\quad$ Befestigung des Rührwerks an zwei parallelen Vierkantträgern (entspricht dem Versuchsstand)

- $\quad$ Befestigung auf einer Einspannscheibe

- $\quad$ Befestigung des Rührwerks auf einem Kugelboden

Bei der Bestimmung von Spannungen und Verformungen in Bauteilen werden in der technischen Mechanik die Beanspruchungsarten Zug, Schub, Biegung und Torsion unterschieden. Bei der Untersuchung eines Rührwerks samt Einspannung spielen die Belastungen Zug und Schub nur eine untergeordnete Rolle und können vernachlässigt werden.

\section{Belastungsfall Biegung des Einspannbalkens}

Ausgehend davon, dass das Rührwerk an einem Balken befestigt ist, wird zunächst dieser Einspannbalken separat untersucht und seine Steifigkeit bestimmt.

Sind der Verlauf des Momentes $M$ und die Biegesteifigkeit bekannt, ist es möglich durch Integration der Differentialgleichung der Biegelinie die Neigung $w_{E}{ }^{\prime}(x)$ und die Durchbiegung $w_{E}(x) \mathrm{zu}$ berechnen. Die Integration kann lediglich bei statisch bestimmt gelagerten Balken durchgeführt werden, da nur in diesem Fall der Momentenverlauf vorab aus den Gleichgewichtsbedingungen ermittelbar ist.

Mit Hilfe des Prinzips der Superposition kann die Durchbiegung bei statisch überbestimmt gelagerten Balken ermittelt werden. Die Differentialgleichung der 
Biegelinie ist im betrachteten Belastungsfall linear. Das bedeutet, dass die Lösung für den Belastungsfall superpositioniert werden kann.
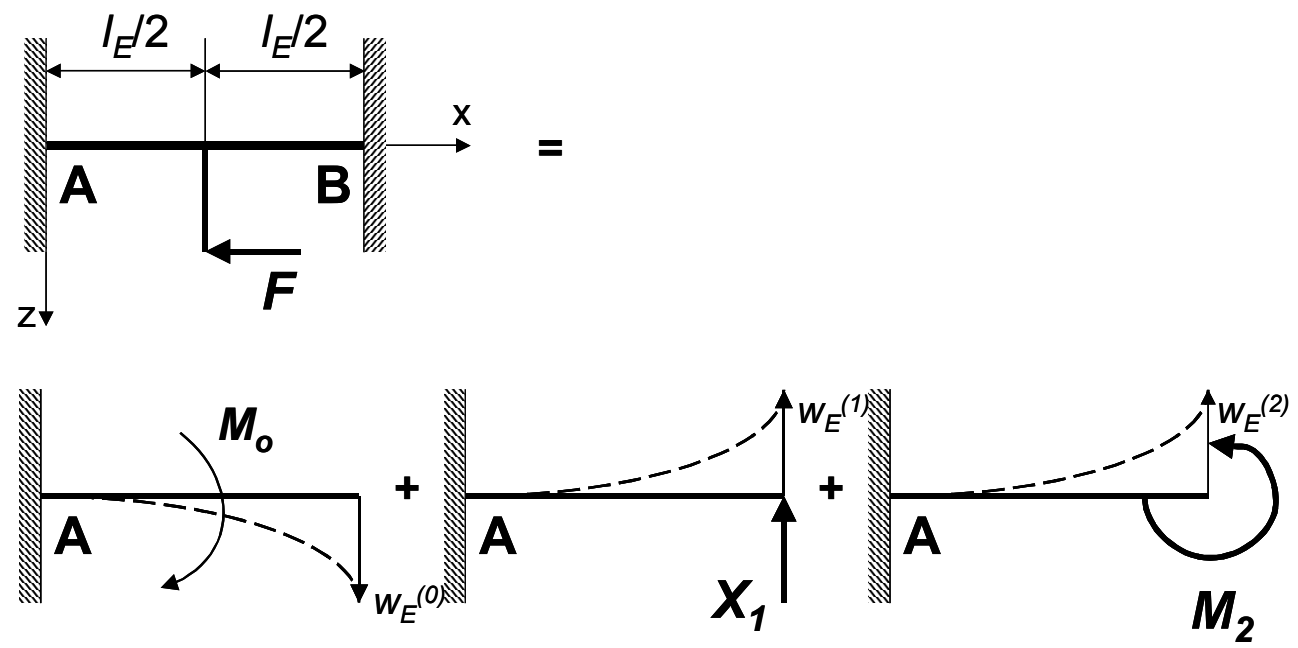

Abbildung 3.20: Biegebelastung und Superposition

Der obere Teil von Abbildung 3.20 zeigt den Balken in seiner Ausgangsposition. Er ist einfach statisch überbestimmt gelagert. Wird das rechte Festlager B entfernt, so wird der Balken statisch bestimmt. Die Neigung $w_{E}{ }^{\prime(0)}$ und die daraus resultierende Verschiebung $w_{E}^{(0)}$ für dieses Grundsystem oder 0-System ergibt sich zu:

$w_{E}^{\prime(0)}=\frac{M_{0} \cdot l_{E}}{2 \cdot E_{E} \cdot I_{E}}$

$w_{E}^{(0)}=\frac{M_{0} \cdot l_{E}^{2}}{2 \cdot E_{E} \cdot I_{E}}\left[1-\left(1-\frac{1}{2}\right)^{2}\right]=\frac{3}{8} \frac{M_{0} \cdot l_{E}^{2}}{E_{E} \cdot I_{E}}$

Der Balken wird nun in einem 1-System durch eine noch unbekannte Kraft $X_{1}$ an der Stelle, an der das Lager B entfernt wurde, belastet. Sie entspricht der Lagerkraft im ursprünglichen System.

Für die Neigung $w^{\prime(1)}$ und die Verschiebung $w^{(1)}$ an dieser Stelle folgt [6]:

$w_{E}^{\prime(1)}=-\frac{X_{1} \cdot l_{E}^{2}}{2 \cdot E_{E} \cdot I_{E}}$

$w_{E}^{(1)}=-\frac{X_{1} \cdot l_{E}^{3}}{3 \cdot E_{E} \cdot I_{E}}$

Das Festlager B auf der rechten Seite kann neben der Lagerkraft $X_{I}$ auch eine Moment $M_{2}$ übertragen. Für das Moment $M_{2}$ gilt [6]: 


$$
\begin{aligned}
& w_{E}^{\prime(2)}=-\frac{M_{2} \cdot l_{E}}{E_{E} \cdot I_{E}} \\
& w_{E}^{(2)}=-\frac{M_{2} \cdot l_{E}^{2}}{2 \cdot E_{E} \cdot I_{E}}
\end{aligned}
$$

Das ursprüngliche System kann als Überlagerung der Lastfälle aus dem 0-, 1- und 2System angesehen werden. Da sich auf der rechten Seite ein Lager befindet, kann an dieser Stelle keine Durchbiegung auftreten. Damit die Lastfälle dem ursprünglichen System entsprechen, muss die Neigung am Festlager B auf der rechten Seite ebenfalls verschwinden:

$$
\begin{aligned}
& w_{E}^{(0)}+w_{E}^{(1)}+w_{E}^{(2)}=0 \\
& w_{E}^{\prime(0)}+w_{E}^{\prime(1)}+w_{E}^{(2)}=0
\end{aligned}
$$

Das Einsetzen in die Kompatibilitätsbedingungen liefert

$$
\begin{aligned}
& X_{1}=\frac{2}{3} \frac{M_{0}}{l_{E}} \\
& M_{2}=-\frac{1}{4} M_{0} .
\end{aligned}
$$

Die Auslenkung der Rührerwelle $w_{\text {Welle }}$ berechnet sich folgendermaßen:

$$
w_{\text {Welle }}=l_{\text {Welle }} \cdot w_{E}^{\prime}\left(\frac{l_{E}}{2}\right)=\frac{1}{16} \frac{M \cdot l_{E} \cdot l_{\text {Welle }}}{E_{E} \cdot I_{E}}
$$

Mit:

$$
\begin{aligned}
& w_{E}(x)=\frac{M \cdot l_{E}^{2}}{2 \cdot E_{E} \cdot I_{E}}\left[\left(\frac{x}{l_{E}}\right)^{2}-\left\{\frac{x}{l_{E}}-\frac{1}{2}\right\}^{2}\right]-\frac{3}{2} \frac{M}{l_{E}} \frac{l^{3}}{6}\left[3\left(\frac{x}{l_{E}}\right)^{2}-\left(\frac{x}{l_{E}}\right)^{3}+\left\{\frac{x}{l_{E}}-1\right\}^{3}\right] \\
& +\frac{1}{4} M \frac{l^{2}}{2}\left[\left(\frac{x}{l_{E}}\right)^{2}-\left\{\frac{x}{l_{E}}-1\right\}^{2}\right] \\
& w_{E}(x)=M l_{\text {Welle }}^{2}\left[\left(\frac{x}{l_{\text {Welle }}}\right)^{2}\left(\frac{1}{2}-\frac{3}{4}+\frac{1}{8}\right)+\frac{1}{4}\left(\frac{x}{l_{\text {Welle }}}\right)^{3}-\frac{1}{2}\left\{\frac{x}{l_{\text {Welle }}}-\frac{1}{2}\right\}^{2}\right]-\left\{\frac{x}{l_{\text {Welle }}}-1\right\}^{3} \\
& w_{E}^{\prime}(x)=\frac{M \cdot l_{E}^{2}}{E_{E} \cdot I_{E}} \frac{1}{l}\left[-\frac{x}{4 \cdot l_{E}}+\frac{3}{4} \frac{x^{2}}{l_{E}^{2}}-\left\{\frac{x}{l_{E}}-\frac{1}{2}\right\}\right]
\end{aligned}
$$




$$
w_{E}^{\prime}\left(\frac{l_{E}}{2}\right)=\frac{1}{16} \frac{M \cdot l_{E}}{E_{E} \cdot I_{E}}
$$

Die Steifigkeit $c_{E}$ der Einspannung bei Biegebelastung ergibt sich zu [6]:

$$
c_{E}=\frac{F}{w_{\text {Welle }}}=\frac{F \cdot 16 \cdot E_{E} \cdot I_{E}}{M \cdot l_{E} \cdot l_{\text {Welle }}}=\frac{F \cdot 16 \cdot E_{E} \cdot I_{E}}{F \cdot l_{\text {Welle }} \cdot l_{E} \cdot l_{\text {Welle }}}=\frac{16 \cdot E_{E} \cdot I_{E}}{l_{\text {Welle }}{ }^{2} \cdot l_{E}}
$$

mit

$$
M=F \cdot l_{\text {Welle }} \text {. }
$$

Abbildung 3.21 zeigt den Balken in ausgelenkter Position.

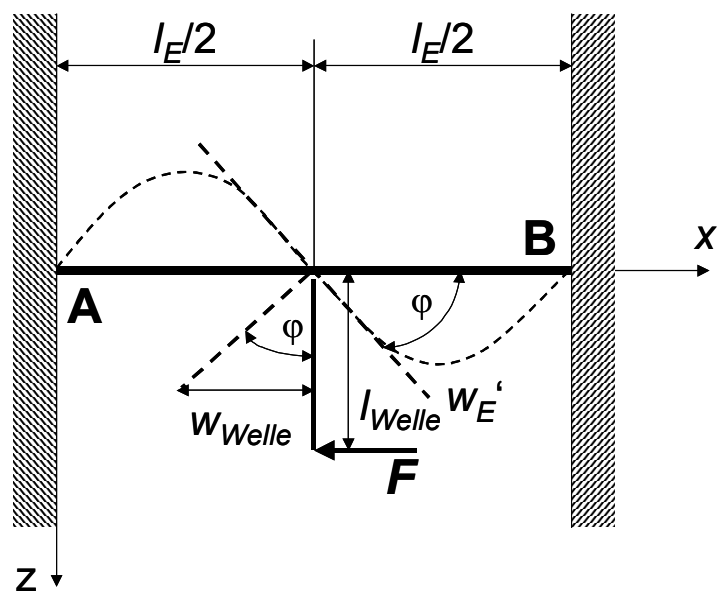

Abbildung 3.21: Balkenauslenkung 


\section{Belastungsfall Torsion des Einspannbalkens}

Wird ein Balken durch Kräfte quer zu seiner Längsachse oder durch Momente um Achsen, die senkrecht zur Längsachse stehen, belastet, so überträgt er Querkräfte und Biegemomente [95].

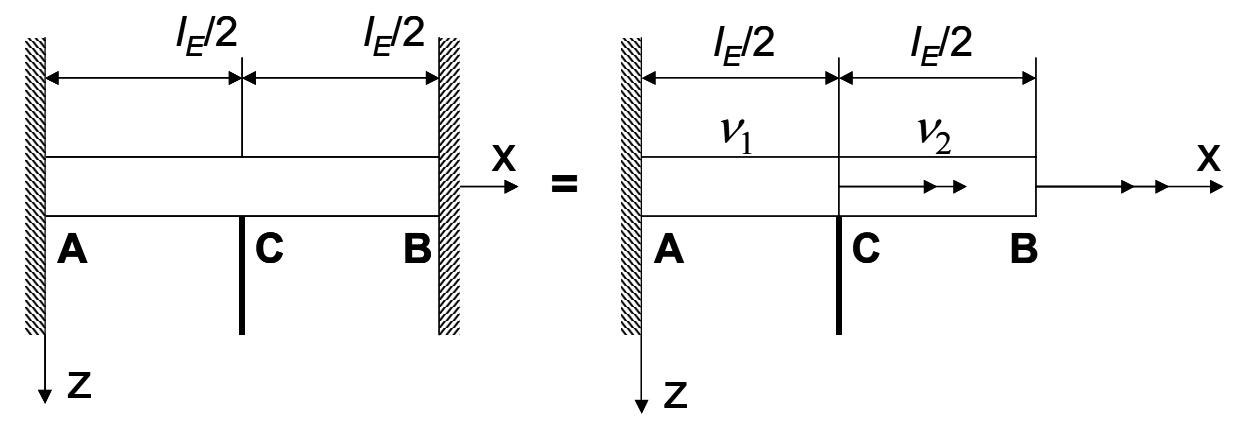

Abbildung 3.22: Torsionsbelastung

Im betrachteten Belastungsfall der Einspannung einer Rührerwelle wirkt zusätzlich ein äußeres Moment, das um die Längsachse der Einspannung wirkt. Diese Belastung verdreht oder tordiert den Balken. Im Balken tritt ein Torsionsmoment auf.

Der beidseitig eingespannte Balken in Abbildung 3.22 ist statisch überbestimmt. Der Drehwinkel $\vartheta$ wird durch abschnittsweise Integration ermittelt. Da der Balken auf der rechten Seite fest eingespannt ist, verschwindet hier der Drehwinkel $\vartheta_{B}$.

$\vartheta_{B}=0=\vartheta_{1}+\vartheta_{2}=\frac{l}{2} \frac{1}{G \cdot I_{T}}[(M+x)+x]$

Aus dem Momentenverlauf ergibt sich:

$M+2 x=0$ bzw. $x=-\frac{M}{2}$

Der Drehwinkel $\vartheta_{1}$ an der Stelle der Momenteinleitung berechnet sich zu:

$\vartheta_{1}=\vartheta_{\frac{l}{2}}=\frac{l}{2} \frac{1}{G \cdot I_{T}}(M+x)=\frac{l_{E}}{2} \frac{1}{G \cdot I_{T}}\left(M-\frac{M}{2}\right)=\frac{l_{E} \cdot M}{4 \cdot G \cdot I_{T}}$

Die Durchbiegung $w_{E}$ der Einspannung beträgt:

$w_{E}=\vartheta_{\frac{l}{2}} \cdot l_{\text {Welle }}$

Für die Steifigkeit der Einspannung gegenüber Torsion erhält man: 
$c_{E, T}=\frac{F}{w_{E}}=\frac{F \cdot 4 \cdot G \cdot I_{T}}{l_{\text {Welle }} \cdot l_{E} \cdot F \cdot l_{\text {Welle }}}=\frac{4 \cdot G \cdot I_{T}}{l_{E} \cdot l_{\text {Welle }}^{2}}$

mit $M=F \cdot l_{\text {Welle }}$.

\section{Ersatzmodell für das System Rührwerk-Einspannträger}

Das gesamte Rührersystem, bestehend aus Einspannvorrichtung und Rührwerk, wird als eine Reihenschaltung von zwei ungedämpften Federelementen angesehen.

Für die Gesamtsteifigkeit in Reihe geschalteter ungedämpfter Federelemente gilt:

$\frac{1}{c_{\text {ges }}}=\sum \frac{1}{c_{i}}$

Die Steifigkeit einer Welle berechnet sich zu

$c_{\text {Welle }}=\frac{3 \cdot E_{\text {Welle }} \cdot I_{\text {Welle }}}{l_{\text {Welle }}^{3}}$

Als Maß für die Steifigkeit des gesamten Rührersystems dient im CFD-Code eine Ersatzwellenlänge des Rührers. Die Steifigkeit des Gesamtsystems wird durch eine Ersatzwellensteifigkeit $l_{\text {Welle,Ersatz }}$ beschrieben. Setzt man die Steifigkeit dieser Ersatzwelle mit der Gesamtsteifigkeit des Rührersystems gleich

$$
\frac{1}{c_{\text {ges }}}=\frac{1}{c_{E}}+\frac{1}{c_{\text {Welle }}}=\frac{l_{\text {Welle,Ersatz }}^{3}}{3 \cdot E_{\text {Welle }} \cdot I_{\text {Welle }}},
$$

erhält man für die Ersatzwellenlänge $l_{\text {Welle,Ersatz }}$ folgende Beziehung

$l_{\text {WelleErsatz }}=\sqrt[3]{3 \cdot E_{\text {Welle }} \cdot I_{\text {Welle }} \cdot\left(\frac{1}{c_{E}}+\frac{1}{c_{\text {Welle }}}\right)}$.

Die in diesen Formeln verwendete Einspannsteifigkeit $c_{E}$ wird für unterschiedliche Einspanngeometrien im nachfolgenden Kapitel bestimmt.

\section{Vierkantprofil}

Die Rührerwelle wird bei dieser Einspannung an zwei parallelen Vierkantträgern befestigt. Die Träger sind als dünnwandige, quadratische Kastenprofile ausgeführt. Es werden zwei unterschiedliche Abmessungen betrachtet:

Der Träger 35/2 weist eine Kantenlänge $b_{1}$ von $35 \mathrm{~mm}$ und eine Wandstärke $b_{1}-b_{2}$ von $2 m m$ auf.

Bei dem Träger mit der Bezeichnung 56/3 beträgt die Kantenlänge $b_{1} 56 \mathrm{~mm}$ und die Wandstärke $b_{1}-b_{2} 3 \mathrm{~mm}$. 
Die Einspannlänge $l_{E}$ beträgt bei beiden Varianten $1,44 m$.

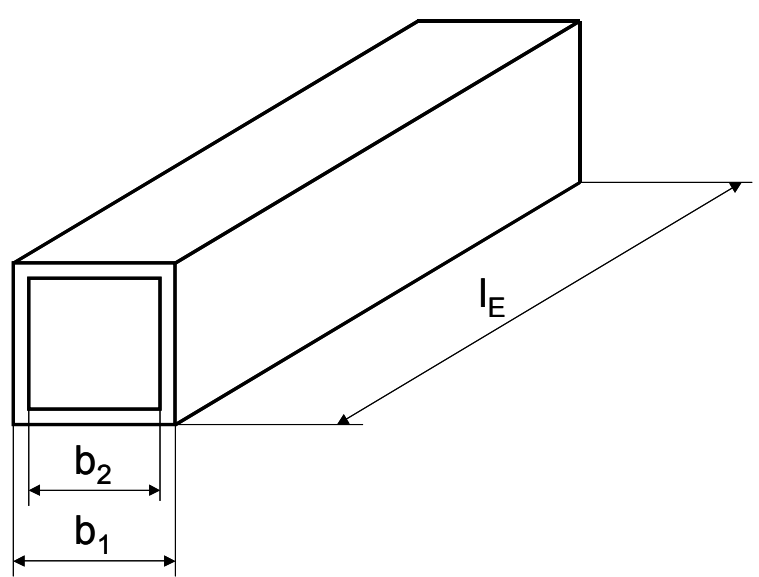

Abbildung 3.23: Vierkantträger

\section{Belastung auf Biegung}

Das Flächenträgheitsmoment eines dünnwandigen, quadratischen Kastenprofils berechnet sich zu [6]:

$$
I_{E}=\frac{b_{1}^{4}-b_{2}^{4}}{12}
$$

Die gesamte Einspannsteifigkeit der beiden parallelen Träger beträgt mit $c_{E}$ aus (3.85)

$c_{E, \text { gesamt }}=\sum c_{E}=2 \cdot c_{E}=2 \cdot \frac{16 \cdot E_{E} \cdot I_{E}}{l_{\text {Welle }}^{2} \cdot l_{E}}=\frac{32 \cdot E_{E} \cdot I_{E}}{l_{\text {Welle }}^{2} \cdot l_{E}}$.

Für die Ersatzwellenlänge $1_{\text {Welle Ersatz }}$ folgt aus (3.95)

$l_{\text {Welle,Ersatz }}=\sqrt[3]{3 \cdot E_{\text {Welle }} \cdot I_{\text {Welle }} \cdot\left(\frac{1}{c_{E, \text { ges }}}+\frac{1}{c_{\text {Welle }}}\right)}$.

Belastung auf Torsion

Das Torsionsträgheitsmoment für einen dünnwandigen, quadratischen Kastenträger beträgt [6]:

$$
I_{T}=\frac{\left(2 A_{m}\right)^{2}}{\oint \frac{d s}{t}}=\frac{4 \cdot b^{2} \cdot h^{2}}{2\left(\frac{b}{t_{b}}+\frac{h}{t_{h}}\right)}=b^{3} \cdot t
$$

Die gesamte Einspannsteifigkeit der beiden parallelen Vierkantprofile beträgt mit $c_{E, T}$ aus (3.89)und (3.91) 


$$
c_{E, \text { ges }, T}=\sum c_{E, T}=2 \cdot c_{E, T}=2 \cdot \frac{4 \cdot G \cdot I_{T}}{l_{E} \cdot l_{\text {Welle }}^{2}}=\frac{8 \cdot G \cdot I_{T}}{l_{E} \cdot l_{\text {Welle }}^{2}} .
$$

Aus (3.95) folgt mit (3.100)

$$
l_{\text {Welle Ersatz }, T}=\sqrt[3]{3 \cdot E_{\text {Welle }} \cdot I_{\text {Welle }, T} \cdot\left(\frac{1}{c_{E, \text { ges }, T}}+\frac{1}{c_{\text {Welle }}}\right)} .
$$

Hat man als Einspannung keinen Balken sondern eine andere Geometrie, wie z.B. eine Aufspannscheibe oder einen Kugelboden, so muss durch Näherungsformeln oder FEM-Simulationen der $c_{E}$-Wert bestimmt und in Gleichung (3.95) eingesetzt werden. 


\subsection{Zusammenfassung Berechnungsverfahren}

Mit dem vorgestellten numerischen Berechnungsverfahren können dynamische Rotorschwingungen mit Fluid-Struktur-Kopplung und verschiedene Einspannsteifigkeiten simuliert werden.

In dieser Arbeit wurde ein kommerzieller CFD-Code durch ein numerisches Modell für die Rührerdynamik erweitert (Fluid-Struktur-Kopplung). Das simulierte Strömungsfeld wird vollständig mit der Rührwerksstruktur gekoppelt. Somit können sämtliche transienten fluiddynamischen Kräfte auf das Rührwerk geltend gemacht werden. Für die Strömungsberechnung kommt ein Finite-Volumen-Verfahren mit verschiebbaren und deformierbaren Kontrollvolumina bei gleichzeitiger Anwendung der ,sliding mesh“-Methode zum Einsatz.

Um die Güte der entwickelten numerischen Berechnungsverfahren zu testen, wurden neben den Berechnungen im Leerlauf auch Untersuchungen in Fluiden durchgeführt. Hierbei zeigte sich, dass das numerische Ersatzmodell für die Rührermechanik eine hohe Rechengenauigkeit bei hoher Stabilität und geringem Rechenzeit- bzw. Speicherplatzbedarf bei Betrieb im Leerlauf und im Fluid hat.

Gerade was Stabilität und Rechenzeit angeht, ist das implementierte numerische Ersatzmodell dem implementierten numerischen Balkenmodell weit überlegen.

Aufgrund der Testrechnungen können folgende Empfehlungen für die numerischen Einstellparameter des entwickelten numerischen Ersatzmodells gemacht werden:

Einen optimalen Kompromiss zwischen Rechenaufwand und Ergebnisgenauigkeit für die untersuchten Rührwerkstypen stellen folgende numerische Einstellparameter dar:

- $\quad k-\varepsilon$ Turbulenzmodell mit 35000 Gitterzellen

- $\quad$ Newmark-Verfahren mit 80TS/U

- 5 Iterationen pro TS im Leerlauf, 10 Iterationen pro TS im Fluid

Zur weiteren Überprüfung des numerischen Ersatzmodells für die Rührermechanik dienen Versuchsdaten, die mit einem Rührwerksversuchsstand gewonnen werden.

Im nächsten Kapitel werden daher die Versuchsanordnung und die Versuchsdurchführung beschrieben, in Kapitel 5 folgt dann der Vergleich der Simulationsergebnisse mit den experimentellen Resultaten. 


\section{Experimentelle Untersuchungen}

Zur Validierung des in Kapitel 3 entwickelten numerischen Berechnungsverfahrens für Rührwerksschwingungen, wird ein Versuchsstand errichtet, in dem folgende Parameter überprüft werden können:

- $\quad$ Leistungseintrag des Rührwerks

- Strömungsgeschwindigkeiten mittels LDA-Messung

- Rührwerksschwingungen

- $\quad$ Drücke auf das Rührerblatt

\subsection{Beschreibung der Versuchseinrichtung und Versuchs- durchführung}

\subsubsection{Versuchsstandübersicht}

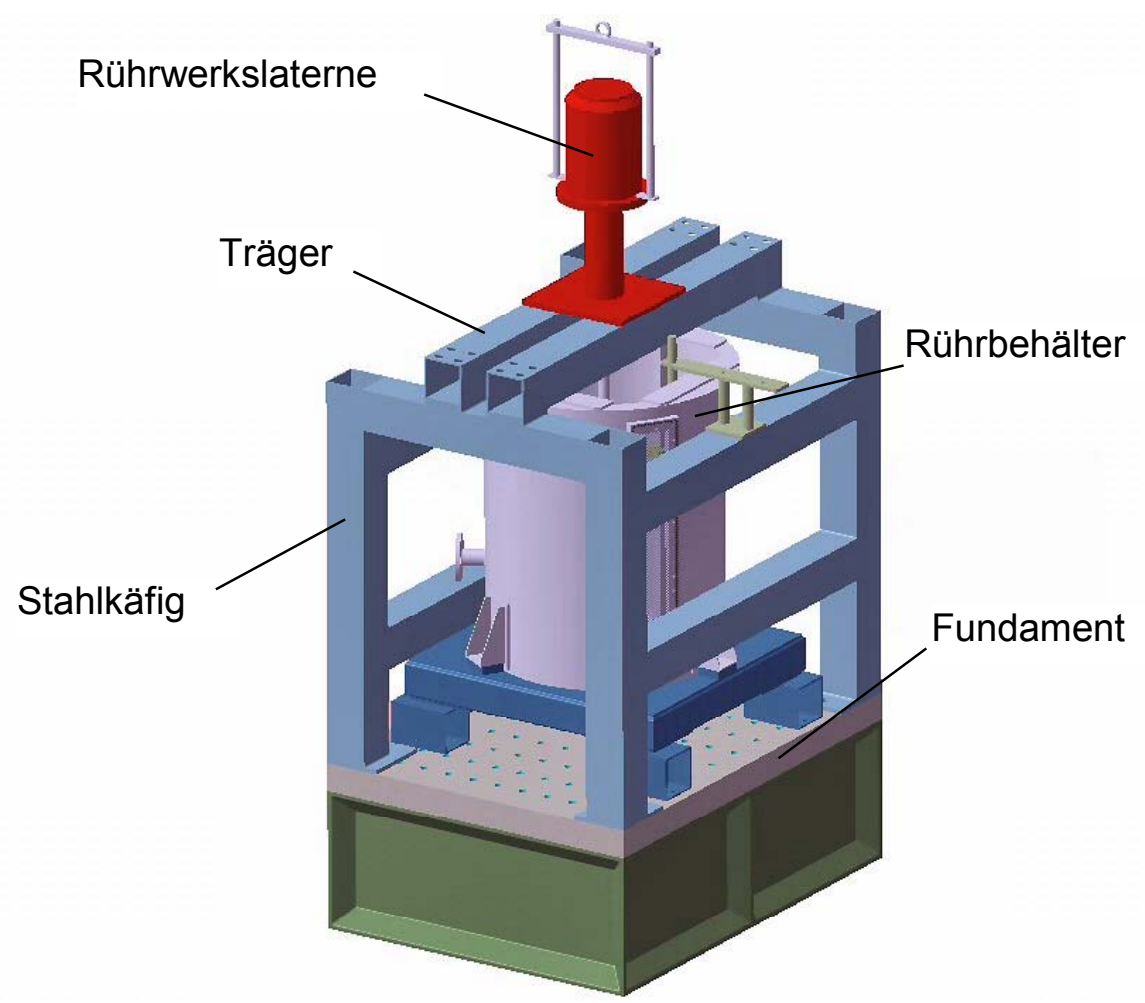

Abbildung 4.1: Versuchsstand

Der Versuchsstand (siehe Abbildung 4.1) besteht aus einem oben offenen Edelstahlbehälter mit zwei integrierten Schaugläsern und folgenden Abmessungen 
- $\quad$ Durchmesser $D=800 \mathrm{~mm}$

- Höhe $H=1200 \mathrm{~mm}$

- $\quad$ Füllhöhe $H_{0}=800 \mathrm{~mm}$

- Klöpperboden

Es ergibt sich somit ein Füllvolumen von 350l. Als Bewehrung können senkrecht zur Behälterwand vier Stromstörer in den Behälter eingebaut werden, welche jeweils eine Breite von $100 \mathrm{~mm}$ aufweisen. Zur Vermeidung von Staupunkten wurde ein Abstand von $20 \mathrm{~mm}$ zwischen Stromstörer und Behälterwand gewählt. Die Stromstörer sind ebenfalls aus Edelstahl.

Das Rührwerk FKL 40, welches von der Fluid Misch- und Dispergiertechnik GmbH zur Verfügung gestellt wurde, setzt sich aus dem Antriebsmotor Elektra mit einer Leistung $P$ von $5,5 \mathrm{~kW}\left(n_{\max }=1500 \mathrm{U} / \mathrm{min}\right)$, der Lagerlaterne mit Präzisionsrillenkugellager 6014 [99] mit 14 Kugeln und der Rührerwelle (Vollwelle) zusammen.

Die frei fliegende Rührerwelle kann folgendermaßen beschrieben werden:

- $\quad$ frei fliegende Länge $l_{\text {Welle }}=1,34 m$

- Durchmesser $d_{\text {Welle }}=40 \mathrm{~mm}$

- $\quad$ Flächenträgheitsmoment der Welle $I_{y}=1,257 \mathrm{E}-07 \mathrm{~m}^{4}$

- $\quad$ Elastizitätsmodul $E=2,1 \mathrm{E} 11 \mathrm{~N} / \mathrm{m}^{2}$

Über einen Frequenzumformer besteht die Möglichkeit, die Drehfrequenz stufenlos zu variieren. 


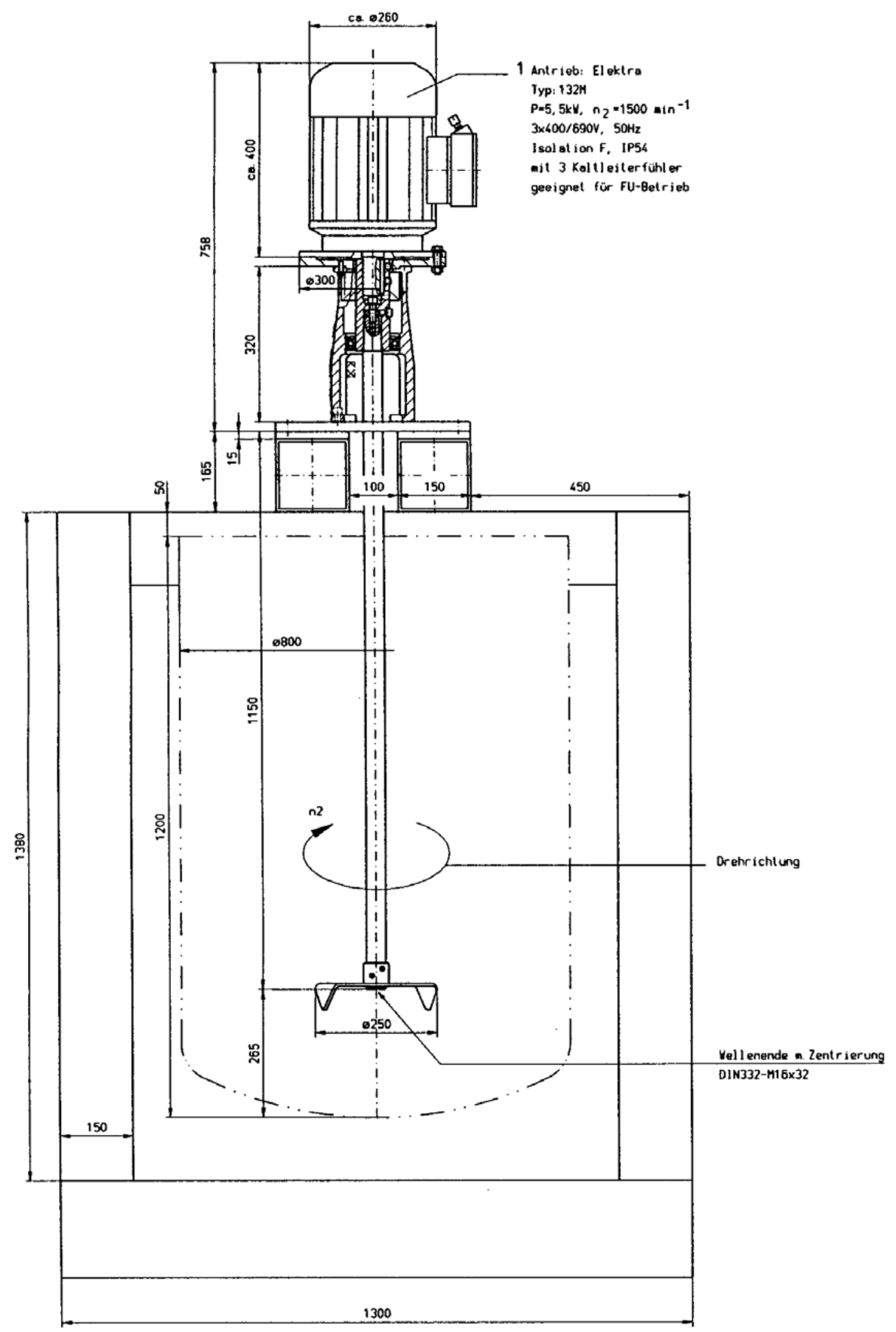

Abbildung 4.2: Geometrische Daten des Rührbehälters 
In Abbildung 4.3 und Abbildung 4.4 sind die in der Arbeit eingesetzten Rührorgane dargestellt:

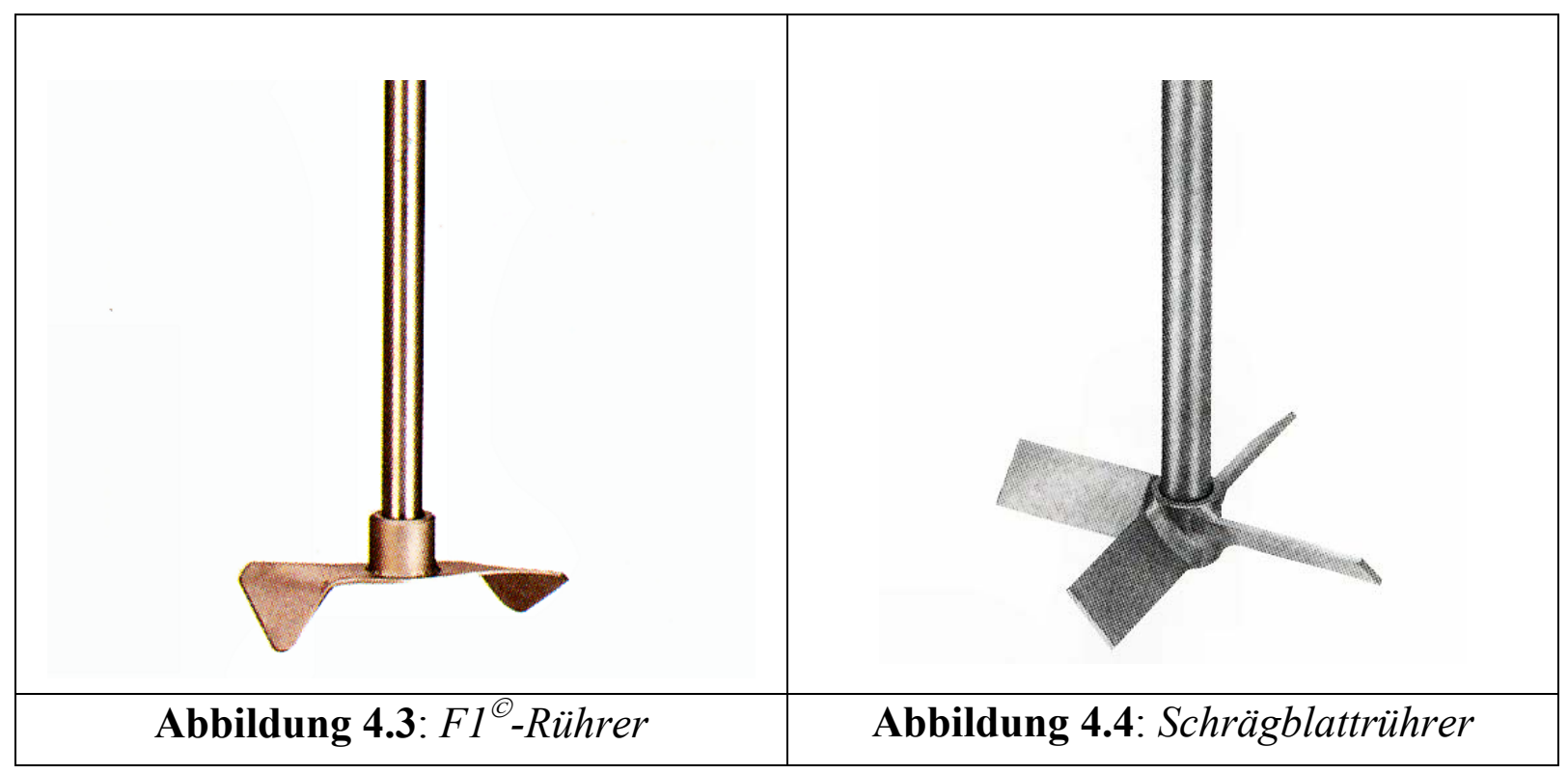

Der verwendete axialfördernde $\quad \mathrm{F} 1^{\odot}$-Rührer der Firma Fluid Misch- und Dispergiertechnik GmbH wurde gewählt, da er ein ähnliches Strömungsfeld aufweist wie ein Propellerrührer.

Die geometrischen Daten der beiden untersuchten Rührertypen sind in folgender Tabelle 4.1 zusammengefasst:

\begin{tabular}{|c|c|c|c|c||}
\hline \hline Rührorgan & $\begin{array}{c}\text { Durchmesser } \\
\boldsymbol{d}_{\text {Rührer }}\end{array}$ & Blattzahl $\boldsymbol{N}_{\boldsymbol{B}}$ & Anstellwinkel & $\begin{array}{c}\text { Masse } \\
\boldsymbol{m}_{\text {Rührer }}\end{array}$ \\
\hline Schrägblattrührer & $0,25 \mathrm{~m}$ & 4 & $45^{\circ}$ & $0,52 \mathrm{~kg}$ \\
\hline $\boldsymbol{F 1 ^ { \odot }}$-Rührer & $0,275 \mathrm{~m}$ & 2 & $45^{\circ}$ & $0,77 \mathrm{~kg}$ \\
\hline
\end{tabular}

Tabelle 4.1: Geometrische Daten der beiden verwendeten Rührertypen

Beide Rührertypen sind aus Edelstahl 1.4571 gefertigt.

Das Rührwerk FKL 40 wird auf einem Stahlkäfig aus Vierkantprofilen befestigt, der nicht direkt mit dem Rührbehälter verbunden ist. Somit ist sichergestellt, dass keinerlei Vibrationen vom Rührbehälter auf den Käfig, sowie vom Stahlkäfig auf den Rührbehälter übertragen werden. 
Der Stahlkäfig besteht aus zusammengeschweißten Vierkantprofilen 150/6 mit einer Kantenlänge von $150 \mathrm{~mm}$ und einer Wandstärke von $6 \mathrm{~mm}$, die mit Schrauben M 30 auf einer stabilen Fundamentlochplatte befestigt sind. Diese äußerst robuste Ausführung wird gewählt, um ausschließlich die Rührwellenschwingungen messen zu können. Störende Schwingungen des Käfigs würden, wie die Behälterschwingungen, eine Verfälschung des Messergebnisses zur Folge haben.

Durch die äußerst massive Bauweise kann bei diesem Versuchsstand von einer starren Einspannung des Rührwerks ausgegangen werden.

\subsubsection{Versuchstechnik und Durchführung}

Beim Versuch, im Leerlauf in den Drehzahlbereich der Selbstzentrierung zu fahren, kam es zu einem Schadensfall, der in Kapitel 1 beschrieben wurde. Aus Sicherheitsgründen werden deshalb beide Rührwerke bei sämtlichen Versuchsreihen nicht über 750U/min betrieben.

In eigenen vorangegangenen Arbeiten [71], [91] wurde festgestellt, dass das Strömungsfeld bei beiden Rührorganen aus dem Stillstand nach ca. $125 \mathrm{U}$ oder $20 \mathrm{~s}$ bei $n=400 \mathrm{U} / \mathrm{min}$ quasistationär vollständig eingelaufen ist. Aus Genauigkeitsgründen wird deshalb auch bei allen Versuchen nach jeder Drehzahlsteigerung mindestens 20s gewartet, bis die jeweiligen Messungen durchgeführt werden.

\section{Leistungseintrag des Rührwerks}

Eine gängige Möglichkeit, das berechnete, numerische Strömungsfeld zu validieren, bietet der Vergleich des Leistungseintrags $P$ [8], [56], [90].

Der Leistungseintrag im Experiment wird über ein Drehmomentmessgerät $D 166 P$ der Firma Sensoplan $\mathrm{GmbH}$ bestimmt. Das Messprinzip dieses berührungslosen Drehmomentmessverfahrens beruht auf den Grundgleichungen des Asynchronmotors. Um das im Motor erzeugte Drehmoment berechnen zu können, muss der Statorfluss bekannt sein. Dieser wird aus den gemessenen Werten von Strom und Spannung gewonnen, indem die Drei-Phasen-Größen zunächst in Raumzeiger umgewandelt werden. Durch Transformation dieser Raumzeiger in ein drehfeldfestes Koordinatensystem entstehen Gleichungsgrößen, die für die Flussbestimmung von Vorteil sind. Ausgangspunkt für die weitere Berechnung ist die StatorSpannungsgleichung. Nach der Trennung von Imaginär- und Realteil folgt nachfolgendes Gleichungssystem für die Bestimmung des Ständerflusses $\Psi$ [98] 


$$
\begin{aligned}
& \dot{\Psi}_{1 x}=u_{1 x}-i_{1 x} R_{1}+\omega_{S} \Psi_{1 y} \\
& \dot{\Psi}_{1 y}=u_{1 y}-\omega_{S} \Psi_{1 x}
\end{aligned}
$$

mit Spannung $u$, Strom $i$, Statorwicklungswiderstand $R_{I}$ und Drehfeldfrequenz $\omega_{S}$.

Da der Stromzeiger auf der reellen Achse liegt, ist der Imaginärteil stets Null.

Das im Motor erzeugte Drehmoment kann somit zu

$$
M=\frac{3}{2} p i_{1 x} \Psi_{1 y}
$$

berechnet werden, wobei $p$ für die Polzahl steht.

Mit dem Drehmomentmessgerät ist es möglich, aus den elektrischen Größen Spannung und Strom das Drehmoment mit einer Genauigkeit von 2\% an der Welle eines Asynchronmotors zu bestimmen. Strom und Spannung werden in der Netzzuleitung des Motors mit einem Feldkompensationswandler abgegriffen, wodurch sich das Drehmoment berechnen lässt. Es müssen der Ohmsche Statorwiderstand, die Polzahl sowie die Nenndaten des Motors bekannt sein. Weiterhin sind die Übertragungsfaktoren der Strom/Spannungswandler notwendig.

Der Leistungseintrag des Rührwerks wird schließlich mit $P=M \cdot \omega=M \cdot 2 \pi \cdot n$

bestimmt und über einen Drehzahlbereich von 100U/min bis 600U/min in 100er Schritten gemessen.

\section{Geschwindigkeitsmessung des Strömungsfelds mit LDA}

Beschreibung des LDA-Messverfahrens

Für die Vermessung turbulenter Rührwerksströmungen eignet sich insbesondere die Laser-Doppler-Anemometrie, da sie zum einen in stark instationären und hochturbulenten Strömungsgebieten verlässliche Informationen liefert und zum anderen berührungslos arbeitet, so dass die Strömung nicht beeinflusst wird [28], [87], [88].

Bei dieser Methode wird durch zwei sich kreuzende, kohärente Laserstrahlen ein Interferenzmuster, bestehend aus hell-dunkel-Streifen, erzeugt. Der Abstand dieser Interferenzstreifen $d_{I}$ zueinander ist dabei allein von der Wellenlänge $\lambda$ des Lasers und dem Winkel $\theta$ zwischen den sich kreuzenden Laserstrahlen abhängig und ist folgendermaßen definiert [94]: 


$$
d_{I}=\frac{\lambda}{2 \sin (\theta / 2)}
$$

Für bessere Messergebnisse werden der Flüssigkeit zusätzlich sehr feine Streulichtpartikel zugesetzt, die das Strömungsfeld jedoch nicht beeinflussen. Bewegt sich ein solches Partikel senkrecht durch diese Interferenzmuster, befindet es sich abwechselnd im hellen oder dunklen Bereich. Das vom Partikel reflektierte oder gebeugte Licht besitzt dadurch eine Intensität, die mit einer charakteristischen Frequenz oszilliert. Diese Dopplerfrequenz $f_{D}$ (Frequenzverschiebung) berechnet sich aus dem Quotienten der Geschwindigkeitskomponente u des Partikels senkrecht zum Interferenzmuster und dem Streifenabstand $d_{I}$ zu:

$$
f_{D}=\frac{u}{d_{I}}
$$

Kombiniert man die beiden Gleichungen (3.5) und (3.6), so liefern sie folgenden eindeutigen Zusammenhang zwischen der Dopplerfrequenz $f_{D}$ und der Geschwindigkeit $u$ :

$$
u=\frac{f_{D} \lambda}{2 \sin \left(\frac{\theta}{2}\right)}
$$

Zur Messung der Dopplerfrequenz wird ein Photodetektor verwendet, der entweder im Sendekopf des LDA-Meßsystems (Rückwärtsstreuverfahren) oder auf der gegenüberliegenden Seite des Messvolumens (Vorwärtsstreuverfahren) angeordnet ist. Der Vorteil des Vorwärtsstreuverfahrens ist ein gutes Signal-Rausch-Verhältnis aufgrund der hohen Streulichtintensität. Der Nachteil ist wiederum ein komplizierter Messaufbau, der einen optischen Zugang zum Messvolumen von zwei Seiten verlangt. Das in dieser Arbeit eingesetzte Messverfahren arbeitet mit dem Rückwärtsstreuverfahren, in dem alle Komponenten im frei traversierbaren LDA-Kopf angeordnet sind. Durch empfindliche Photodetektoren und entsprechend höhere Laserleistung lässt sich ebenfalls ein gutes Signal-Rausch-Verhältnis erreichen.

Der Intensitätsverlauf des reflektierten Lichts („,bursts“) während des Durchlaufs eines Partikels durch das Messvolumen wird mit Hilfe einer Fast-Fourier-Transformation in seine Frequenzanteile zerlegt. Dabei tritt die dominierende Dopplerfrequenz deutlich hervor. Aus der Dopplerfrequenz kann allerdings lediglich der Betrag der Geschwindigkeit und nicht ihre Richtung bestimmt werden. Um eine Richtungsinformation zu bekommen, wird ein Laserstrahl mit Braggzellen moduliert, was zu einer Bewegung der Interferenzstreifen senkrecht zu ihrer Normalen führt. Ein 
ruhendes Partikel würde somit ein Messsignal mit dieser Frequenzverschiebung verursachen. Bewegt sich das Partikel jedoch entgegen der Bewegung der Interferenzstreifen, führt dies zu einer Frequenzerhöhung, wohingegen eine Bewegung in die gleiche Richtung zu einem niederfrequenten Messsignal führt. Somit lässt sich eine Aussage über die Richtung der Geschwindigkeit treffen.

Das Messvolumen, d.h. der Bereich, in dem ein Interferenzmuster vorliegt, hat die Form eines Ellipsoides und ein Volumen $V_{D}$, das sowohl vom Durchmesser der Laserstrahlen $d_{e-2}$ als auch vom Winkel $\theta$ gemäß folgender Formel abhängt:

$V_{D}=\frac{\pi d_{e^{-2}}^{3}}{6 \cos \left(\frac{\theta}{2}\right) \sin \left(\frac{\theta}{2}\right)}$

Durch die hohe zeitliche Aufnahmerate im Bereich von einigen Kilohertz sind neben der Messung der mittleren örtlichen Geschwindigkeit auch Aussagen über die örtlichen Schwankungs- und Turbulenzgrößen möglich [25].

Zur Messung der Geschwindigkeitsfelder im Rührbehälter wird in dieser Arbeit ein Laser-Doppler-Anemometer verwendet. Da nur eine 1D-Optik, d.h. nur ein Paar von Laserstrahlen zur Verfügung steht, kann die Geschwindigkeit jeweils nur in einer Richtung gemessen werden. Durch eine $90^{\circ}$-Drehung des Sendekopfes des Lasers wird die axiale Geschwindigkeit (, $u$-velocity") und die tangentiale Geschwindigkeit (,,wvelocity") an den verschiedenen Messpunkten ermittelt. Der Rührbehälter ist für die optische Messung durch eine ebene Duran-Glasscheibe nur begrenzt zugänglich. Die radiale Geschwindigkeit kann aufgrund der Größe der Duran-Glasscheibe und der Tatsache, dass es sich um einen 1D-Laser handelt, nicht vermessen werden.

Der Laser hat die Laserklasse 3B ( $10 \mathrm{~mW}$ Leistung, Farbe: rot) und ist an einer $x-y-z$ Traverse angebracht. Dadurch ist es möglich, bestimmte Punkte im Strömungsfeld anzufahren, um dort LDA-Messungen durchzuführen.

Um Wirbel und damit verbundene Turbulenzen zu erfassen, werden je Messpunkt ca. 200.000 Messwerte aufgenommen, da Wirbel in Bezug auf Zeit und Ort stochastisch auftreten. Die erfassten Geschwindigkeiten sollten dann möglichst gleichmäßig um die gemittelte Geschwindigkeit herum verteilt sein. Dies wird durch Ausgabe eines Histogramms, wie es in Abbildung 4.5 gezeigt ist, geprüft.

Die Abhängigkeit der Strömungsgeschwindigkeit an den Monitorpunkten vom Rührerdrehwinkel wird über einen Drehwinkelaufnehmer und eine erweiterte Messelektronik bestimmt und in den Auswertungen berücksichtigt. 


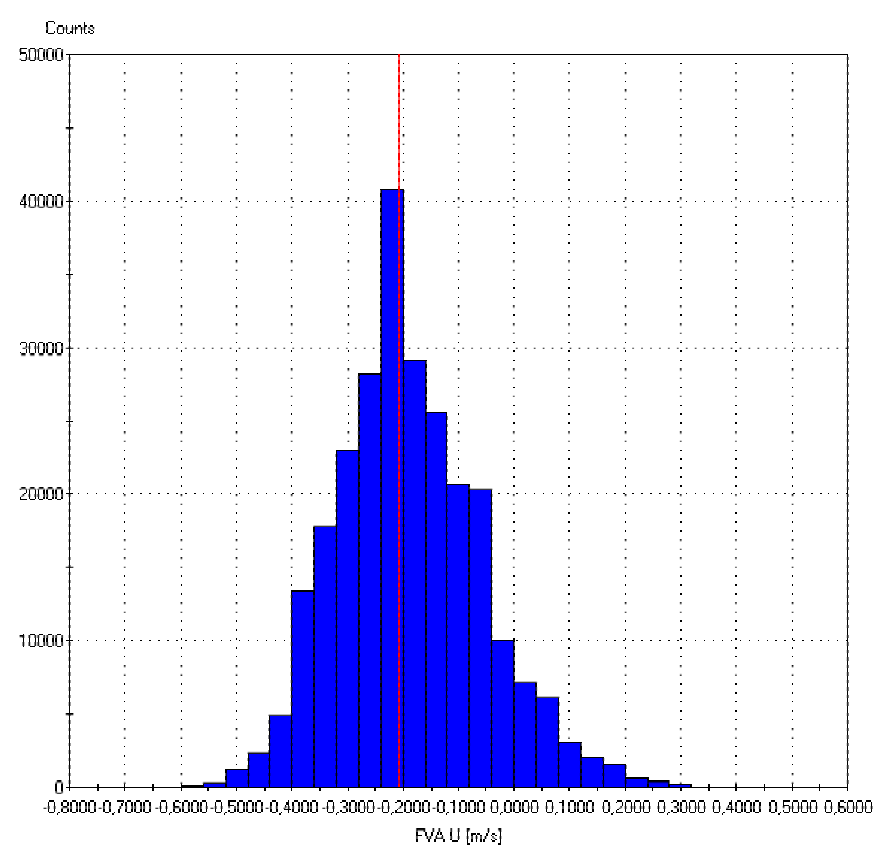

Abbildung 4.5: Histogramm einer LDA-Messung; Anzahl der Messwerte über den gemessenen Geschwindigkeiten

\section{Versuchsdurchführung}

Zunächst gilt es, die Koordinaten der einzelnen Messpunkte so festzulegen, dass sowohl im Experiment als auch in der Simulation exakt dieselben Punkte angefahren bzw. ausgegeben werden können. Abbildung 4.6 zeigt den Aufbau des Rührwerksbehälters und verdeutlicht die Lage der Messpunkte.

Im Experiment ist die Auswahl der Messpunkte durch zwei Faktoren begrenzt. Zum einen durch die Lage des Duranglasfensters, da der übrige Teil des Rührkessels für den Laser undurchlässig ist. Zum anderen durch die Reichweite des Lasers, die zusätzlich durch den Brechungsindex des Mediums beeinflusst wird.

Um die Punkte bei der Simulation exakt ansprechen zu können, sollten sich diese möglichst in der Mitte eines numerischen Gittervolumens befinden und nicht zwischen zwei Zellen liegen. 


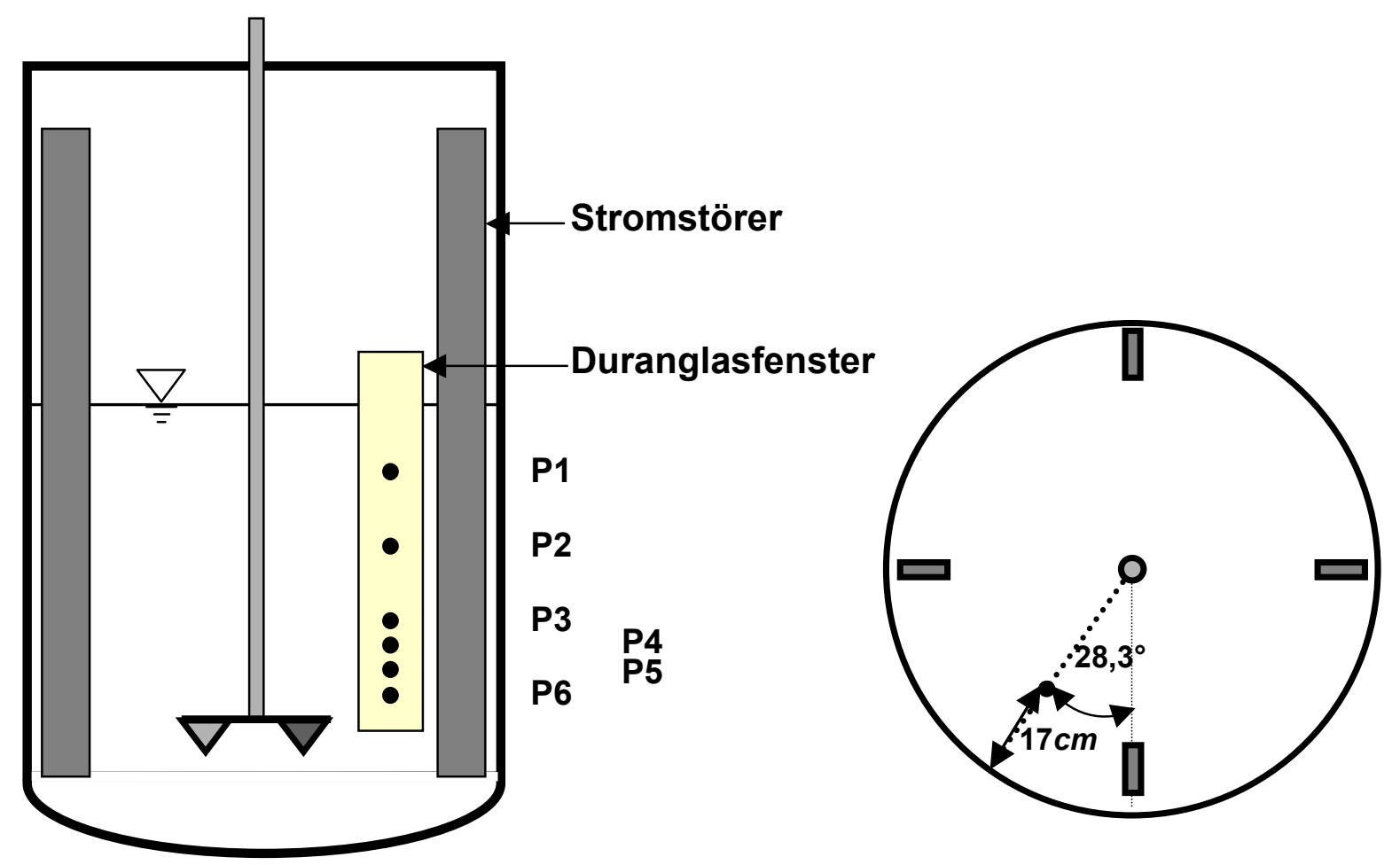

Abbildung 4.6: Rührwerksbehälter in der Vorderansicht (links, F1-Rührer) und Draufsicht (rechts, ohne Rührorgan)

Die vertikale Lage der Messpunkte wird wie folgt festgelegt:

\begin{tabular}{|c|c|c|}
\cline { 2 - 3 } & \multicolumn{2}{c|}{ Abstand zur Wasseroberfläche in $[\mathrm{cm}]$} \\
\cline { 2 - 3 } & $\mathbf{F 1}^{\odot}$-Rührer & Schrägblattrührer \\
\hline \hline Punkt 1 „,P1“ & \multicolumn{2}{|c|}{10} \\
\hline Punkt 2 „,P2“ & \multicolumn{2}{|c|}{20} \\
\hline Punkt 3 „,P3“ & 30 \\
\hline Punkt 4 „,P4“ & 33 & \\
\hline Punkt 5 „,P5“ & 36,5 & 33,6 \\
\hline Punkt 6 „P6“ & \multicolumn{2}{|c|}{40,4} \\
\hline Rührerkopf & \multicolumn{2}{|c|}{54,4} \\
\hline \hline
\end{tabular}

Tabelle 4.2: Axiale Koordinaten der sechs untersuchten Messpunkte

Unterschiedliche Orte von P4 und P5 sind auf unterschiedliche numerische Gitter der beiden simulierten Rührorgane zurückzuführen. 
Die radiale Lage der Messpunkte im Rührbehälter, bzw. die $x$-Komponente der Traversiervorrichtung, wird für die beiden Rührertypen wie folgt ermittelt:

In der Simulation wird der Abstand der Punkte zur Welle des Rührwerks einheitlich auf $23 \mathrm{~cm}$ festgelegt. Bei einem Außendurchmesser $D$ des Rührbehälters von $80 \mathrm{~cm}$ ergibt sich ein Abstand der Punkte zur Außenwand des Rührbehälters von $17 \mathrm{~cm}$. Unter Berücksichtigung des Brechungsindex von Wasser berechnet sich die Laserreichweite von der Wand zum Messpunkt zu $17 \mathrm{~cm} / 1,3 \approx 13 \mathrm{~cm}$. Wie bereits erläutert, ist die radiale Lage der Messpunkte im Rührbehälter hinsichtlich der Simulation begrenzt, da im rotierenden Innenteil (,sliding mesh“) keine ortsfesten Strömungsgrößen ausgegeben werden können.

Als Letztes muss die Position der Punkte in Umfangsrichtung festgelegt werden (siehe hierzu auch Abbildung 4.6). Auch hier ist der Messbereich im Experiment aufgrund der Größe des Duranglasfensters begrenzt. Der Kreisbogen zwischen zwei Strombrechern berechnet sich über $0,25 \cdot U$. Bei einem Innendurchmesser von $79 \mathrm{~cm}$ entspricht das ca. 62 cm. Die Länge des Kreisbogens zwischen Plexiglasfenster und Strombrecher beträgt $14,5 \mathrm{~cm}$ in Strömungsrichtung. Bei einer Breite des Duranglasfensters von $10 \mathrm{~cm}$ und unter Berücksichtigung der Tiefe der Strombrecher von $1 \mathrm{~cm}$ errechnet sich der Abstand zum zweiten Strombrecher zu 36,5cm (entgegen der Strömung). Da der Laser in Umfangsrichtung auf die Mitte des Plexiglasfensters gerichtet ist, ergibt sich ein Abstand von $19,5 \mathrm{~cm}$ oder $28,3^{\circ}$ zum nächsten Strombrecher in Strömungsrichtung.

Dem Experiment entsprechend wird im Simulationsmodell ein in Strömungsrichtung vor dem Strombrecher liegender Block angesprochen. Über eine einfache Dreisatzrechnung wird dann die entsprechende Zelle innerhalb des Blocks ermittelt.

Um die Ergebnisse aus der numerischen Strömungssimulation zu validieren, werden die axialen sowie die tangentialen Strömungsgeschwindigkeiten an sechs verschiedenen Messpunkten mittels LDA-Messung bestimmt.

Die Drehzahlen werden von 100U/min bis $700 \mathrm{U} / \mathrm{min}$ in Schritten von $100 \mathrm{U} / \mathrm{min}$ gesteigert und pro Messpunkt 200.000 Messungen durchgeführt. 


\section{Messung der Rührwerksschwingungen}

$\underline{\text { Beschreibung der Messtechnik }}$

Die Auslenkung der Rührerwelle in Abhängigkeit von der Drehzahl wird transient mit Hilfe einer 2-dimensionalen, laseroptischen Distanzmessung bestimmt.

Der entscheidende Vorteil dieses Messverfahrens ist, dass es sich um ein berührungsloses Messsystem handelt, und sich das Problem des Datentransfers, z.B. von einem Beschleunigungsaufnehmer auf der rotierenden Welle $\mathrm{zu}$ einem Messwerterfassungssystem, nicht stellt.

In Abbildung 4.7 ist die Anordnung der Laserdistanzsensoren im Rührbehälter dargestellt. Sie sind im $90^{\circ}$-Winkel zueinander im gleichen Abstand zur Rührerwelle angeordnet, wodurch man ein zweidimensionales Ergebnis bezüglich der Auslenkung erhält.

Die Laser-Distanz-Sensoren messen die Auslenkung der Rührerwelle knapp oberhalb der Wasseroberfläche (0,64m unterhalb der Einspannung). Die Messrate der Sensoren beträgt $1 \mathrm{kHz}$. Die gemessenen Werte werden mit einem Datenübertragungskabel an ein Spider 8-Messdatenerfassungssystem Firma Hottinger Baldwin Mess- und Systemtechnik GmbH weitergegeben. 


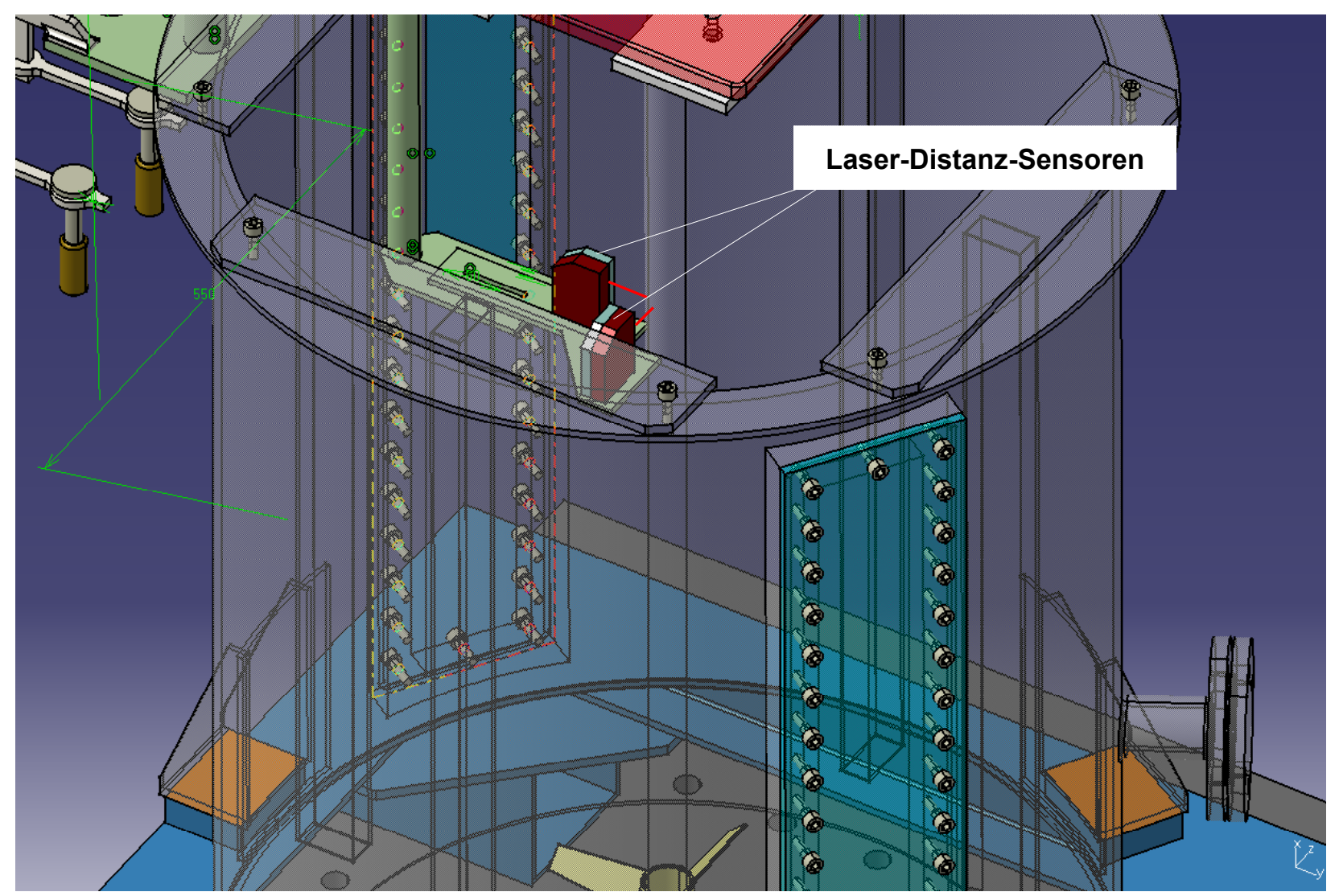

Abbildung 4.7: Anordnung der Laserdistanzsensoren

\section{Beschreibung der Laserdistanzsensoren}

Die Sensoren ILD 1400 arbeiten mit einem Halbleiterlaser der Wellenlänge 670nm (sichtbar/rot). Die maximale Ausgangsleistung beträgt $1 \mathrm{~mW}$. Die Sensoren sind der Laserklasse 2 zugeordnet.

Der Sensortyp ILD1400 arbeitet nach dem Prinzip der optischen Triangulation, d.h. ein sichtbarer Lichtpunkt wird auf die Oberfläche des Messobjektes projiziert. Der diffuse Anteil der Reflexion dieses Lichtpunktes wird von einer Empfängeroptik, die in einem bestimmten Winkel zur optischen Achse des Laserstrahls angeordnet ist, abstandsabhängig auf einem ortsauflösenden Element (CCD-Zeile) abgebildet. Der interne Controller bildet aus dem Zeilensignal den Messwert. Durch eine interne Regelung ist der Sensor in der Lage, gegen unterschiedliche Oberflächen zu messen. Am Lasergehäuse befindet sich zusätzlich eine LED-Anzeige, welche angibt, ob sich das Messobjekt im zulässigen Messbereich $( \pm 20 \mathrm{~mm})$ befindet, oder zu niedrige Reflexion vorliegt. 
Der Laserstrahl muss senkrecht auf die Objektoberfläche treffen, da andernfalls Messunsicherheiten nicht auszuschließen sind.

Prinzipiell wertet der Sensor den diffusen Anteil der Reflexionen des Laserlichtpunktes aus. Eine Aussage über einen Mindestreflexionsgrad ist nur bedingt möglich, da selbst von spiegelnden Flächen noch geringe diffuse Anteile ausgewertet werden können. Dies geschieht durch Intensitätsbestimmung der diffusen Reflexion aus dem Zeilensignal und anschließender Ausregelung von Intensitätsschwankungen.

Verkippungswinkel des Messobjektes sowohl um die $x$ - als auch um die $y$-Achse um weniger als $5^{\circ}$ sind nur bei Oberflächen mit stark direkter Reflexion störend. Verkippungswinkel zwischen $5^{\circ}$ und $15^{\circ}$ bewirken eine scheinbare Abstandsänderung von ca. $0,5 \%$ des Messbereiches.

Ein weiterer Fehlereinfluss ist die Temperatur, weshalb bei Inbetriebnahme eine Einlaufzeit von mindestens $15 \mathrm{~min}$ erforderlich ist, um eine gleichmäßige Temperaturverteilung im Sensor zu erreichen. Für hohe Auflösungen ist ein besonderes Augenmerk auf eine stabile bzw. schwingungsgedämpfte Sensor- und Messobjektmontage zu richten, um mechanische Schwingungen $\mathrm{zu}$ vermeiden. Oberflächenrauigkeiten in der Größenordnung von ca. $5 \mu m$ und darüber, können bei traversierenden Messungen $\mathrm{zu}$ einer scheinbaren Abstandsänderung (einem sogenannten Oberflächenrauschen) führen.

\section{Beschreibung des Messdatenerfassungssystems Spider 8}

Das Messdatenerfassungssystem Spider 8 ist eine PC-Messelektronik für das elektrische Messen mechanischer Größen wie Dehnungen, Kräfte, Drücke, Wege, Beschleunigungen und Temperaturen. Die gesamte Signalkonditionierung - Speisung für passive Aufnehmer und Verstärkung, Digitalisierung, Rechnerinterface und Anschlusstechnik für maximal 8 Kanäle - ist in einem Gehäuse vereinigt. Jeder Kanal arbeitet mit einem eigenen A/D-Wandler, der Messraten von 1/s bis $9600 / s$ erlaubt. Die A/D-Wandler sind synchronisiert, womit ein zeitgleiches Messen auf allen Kanälen sichergestellt wird. Das verwendete Messdatenerfassungssystem besitzt 8 Kanäle, vier davon mit 5-poligen und die anderen vier mit 25-poligen Eingängen. Alle Eingänge können an Stelle von Aufnehmern auch direkt $10 \mathrm{~V}$-Signale verarbeiten. Das Spider 8 ist über eine $R S$-232-Schnittstelle an einen Auswertecomputer angeschlossen. Weiterhin ist am Gerät eine Digital I/O-Buchse (25-polig) vorhanden. Diese stellt acht digitale Eingänge und acht Ein-Ausgänge (8 Bit Input / 8 Bit Output) neben 
Steuersignalen und Zustandsmeldungen zur Verfügung. Alle weiteren erforderlichen Einstellungen werden vom Auswertecomputer per Befehl vorgenommen.

Mit der auf dem Auswertecomputer installierten Spider 8-Software können die I/OKanäle und Datenquellen definiert sowie die einzelnen Kanaleinstellungen vorgenommen werden. Mit dem Setup-Programm lässt sich das Gerät konfigurieren, die aktuellen Messwerte können angezeigt werden. Nach der Konfiguration können die Einstellungen gespeichert und zu einem späteren Zeitpunkt wieder abgerufen und modifiziert werden.

\section{Versuchsdurchführung}

$\mathrm{Zu}$ Beginn der Versuchsreihen wird die Rührerwelle ohne Rührorgan auf geometrische Ungenauigkeiten hin untersucht. Laut Fluid Misch- und Dispergiertechnik GmbH können Auslenkungen von $0,1 \mathrm{~mm}$ auf $1 \mathrm{~m}$ Rührerwelle toleriert werden. Der sogenannte „Schlag“ der Welle wird manuell mit einer Messuhr untersucht und gegebenenfalls durch leichtes Drücken gegen das Wellenende korrigiert.

Zur Ermittlung der Eigenfrequenz der Rührerwelle mit den beiden zu untersuchenden Rührorganen werden zunächst Ausschwingversuche in Luft durchgeführt. Hierzu wird dem Rührwerk ein leichter Schlag versetzt und die Amplituden des Rührwerks über der Zeit aufgezeichnet. In diesem Zusammenhang kann auch die Materialdämpfungskonstante $\varsigma$ ermittelt werden.

Zur Ermittlung der dynamischen Auslenkung der Rührerwelle wird die Drehzahl in Luft und Wasser mit beiden Rührorganen in 50er Schritten von 100U/min an aufwärts gesteigert.

Die Distanzmessungen werden mit unterschiedlichen Abtastfrequenzen $(300 \mathrm{~Hz}, 600 \mathrm{~Hz}$ und $1200 \mathrm{~Hz}$ ) durchgeführt. Die Abtastrate $1200 \mathrm{~Hz}$ ist die genaueste. Hier wird beispielsweise bei einer Drehzahl von 600U/min alle $3^{\circ}$ gemessen. Zusätzlich wird bei den Versuchsreihen mit $1200 \mathrm{~Hz}$ der Besselfilter zwischen $75 \mathrm{~Hz}$ und $20 \mathrm{~Hz}$ variiert.

Im Drehzahlbereich um den unterkritischen Peak (180U/min - 300U/min) wird zur genaueren Auflösung die Drehzahl nur um 10U/min gesteigert. Zur Untersuchung des Einflusses der Strombrecher auf den unterkritischen Peak (siehe Abbildung 2.13) werden in Wasser Versuchsreihen mit vier, einem und ohne Strombrecher durchgeführt.

Um aussagekräftige Ergebnisse zu erhalten, zur Überprüfung der Reproduzierbarkeit und um den Einfluss von Messausreißern zu minimieren, werden die experimentellen Reihen bis zu dreimal wiederholt. 


\section{Druckmessungen an den Rührerblättern}

$\underline{\text { Beschreibung der Messtechnik }}$

Die einzelnen Drücke an den Rührblättern werden mit $0,4 \mathrm{~mm}$ flachen AbsolutDruckaufnehmern der Firma Kulite Semi-Conductor GmbH zeitabhängig gemessen. Die Druckaufnehmer, in die eine aktive DMS-Vollbrücke in eine Membran aus Silizium eindiffundiert ist, haben folgende Abmessungen

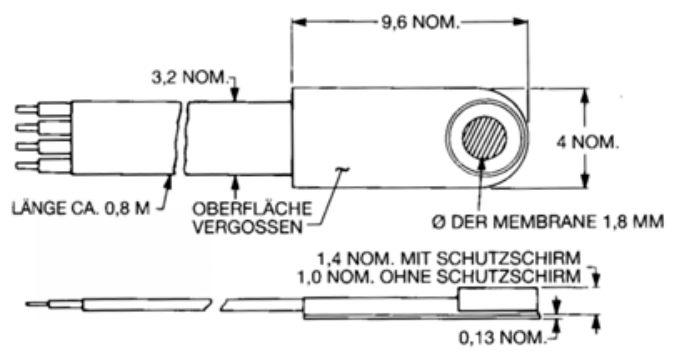

Abbildung 4.8: Absolutdruckaufnehmer

Es werden acht Absolutdruckaufnehmer (D1 - D8) an den Ober- und Unterseiten der Rührerblätter Blatt 3 und Blatt 4 (siehe Abbildung 4.9) mit einem Spezialkleber appliziert und die elektrischen Anschlüsse mit Epoxidharz wasserfest abgedichtet. Die Druckaufnehmer werden nahe der äußeren Rührerblätterecken angebracht. Hier treten die größten Geschwindigkeiten auf. Blatt 3 wird gewählt, da in dieser Richtung aufgrund der Exzentrizität der Schwerpunkt des Rührwerks liegt.

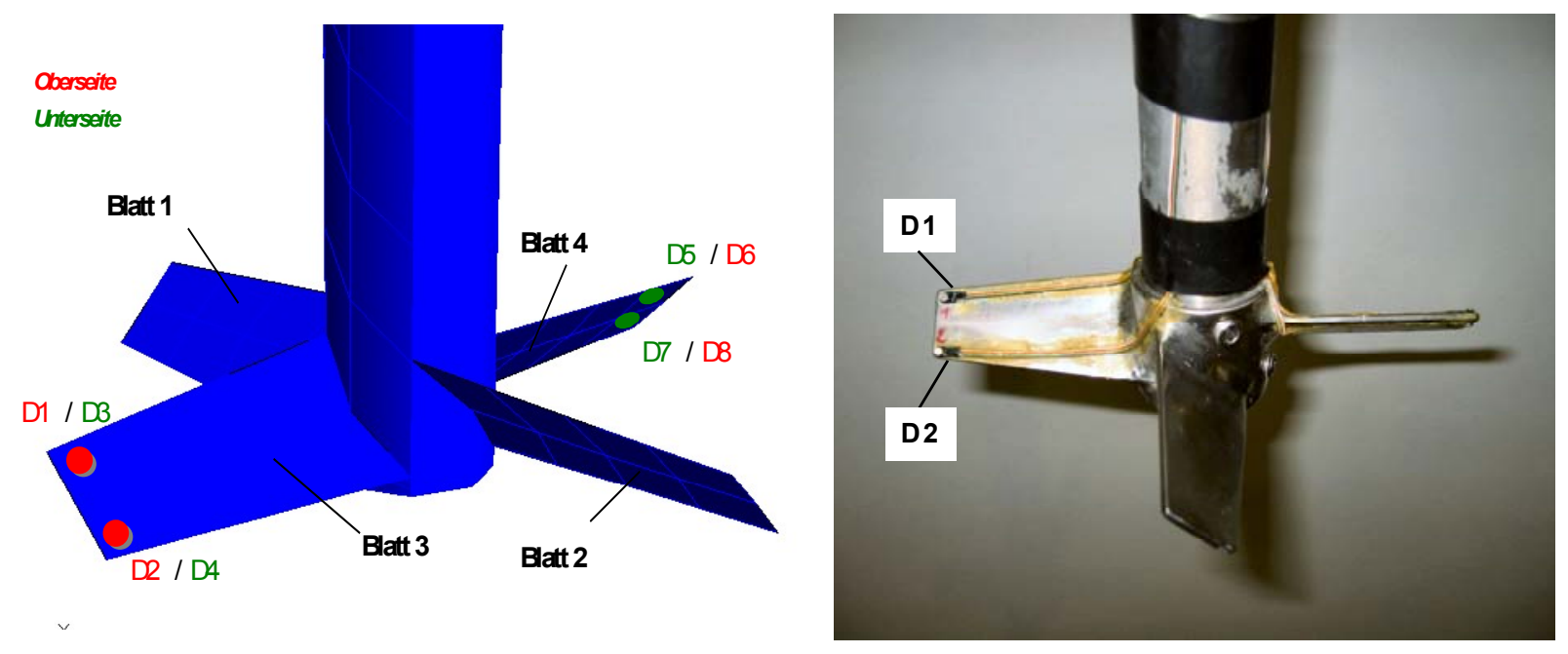

Abbildung 4.9: Anordnung der Druckaufnehmer auf den Rührerblättern 
Die Druckaufnehmer können maximal bis 1,7bar Absolutdruck belastet werden. Von den Druckaufnehmern führen jeweils zwei Input und Output Kabel zum Sender. Die Kabel werden entlang der Welle zum Senderträger geführt, der weiter oben an der Rührerwelle angebracht ist, um nicht in Kontakt mit Wasser zu kommen. Die Kabel sind entlang der Welle mit Gummiringen befestigt und mit Klebeband fixiert. An den Rührerblättern werden sie zusätzlich mit einem Harzkleber befestigt.

\section{$\underline{\text { Telemetriesystem }}$}

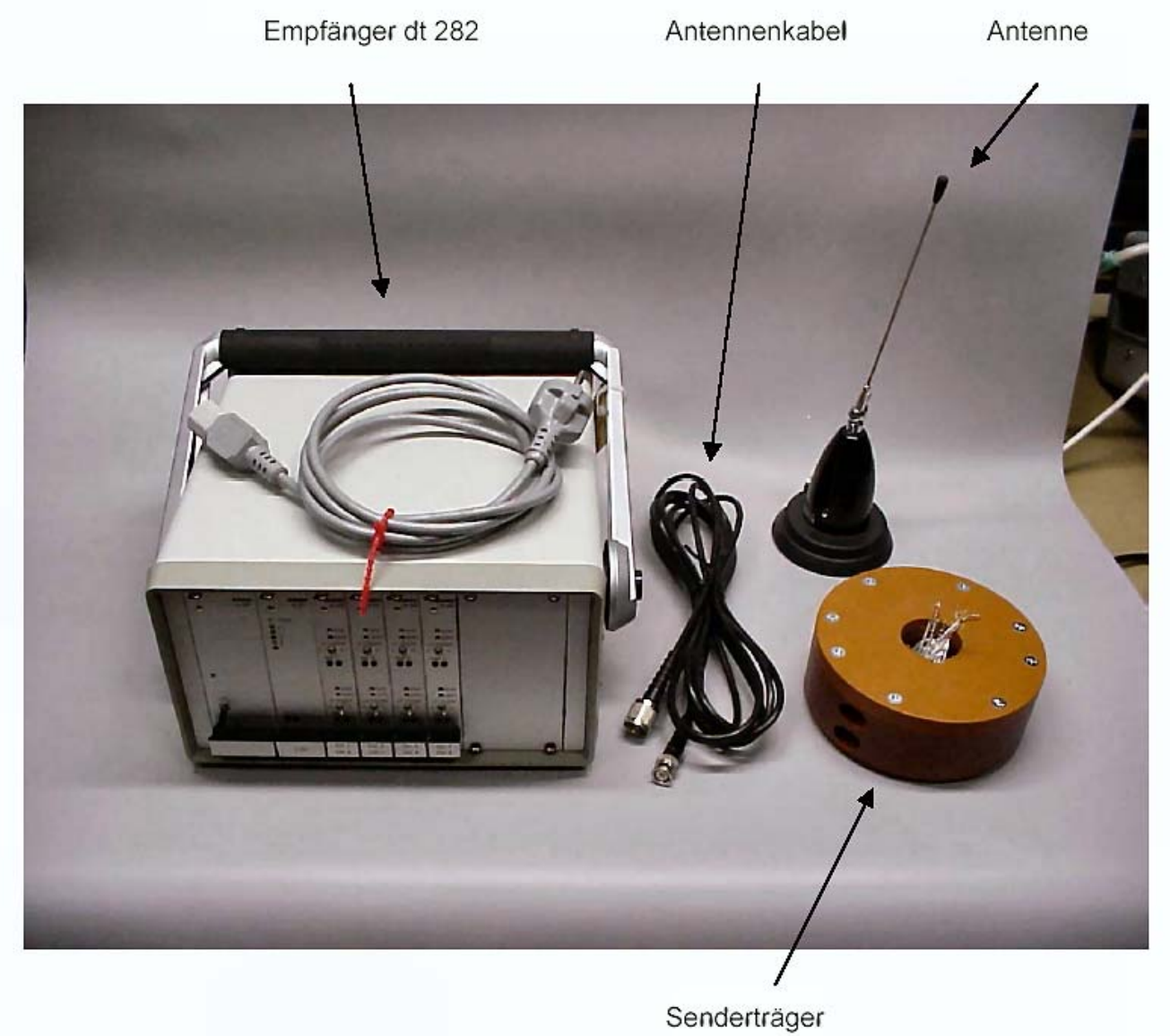

Abbildung 4.10: Darstellung der Telemetriesystemkomponenten

Zur kontaktlosen Weiterleitung der Messdaten an das Messdatenerfassungssystem Spider 8 wird ein 8-Kanal Telemetriesystem der Firma Telemetrie Elektronik GmbH verwendet. Dieses System hat eine Signalbreite von 0 bis $1 \mathrm{kHz}$ pro Kanal und stellt eine absolut zeitgleiche Übertragung aller Messkanäle sicher. Es besteht aus einem sonderangefertigten, rotationssymmetrischen Senderträger mit integriertem 
Sendemodul, Senderantenne und Stromversorgung, der Empfangsantenne und der Empfangseinheit, die in ein Tischgehäuse integriert ist.

Der zweiteilige Senderträger aus $H G W 2082$ (Novotex) (siehe Abbildung 4.11) wird auf die Rührerwelle aufgespannt. Der untere Teil nimmt 6 AA-Batterien auf, der obere Teil das Sendemodul.
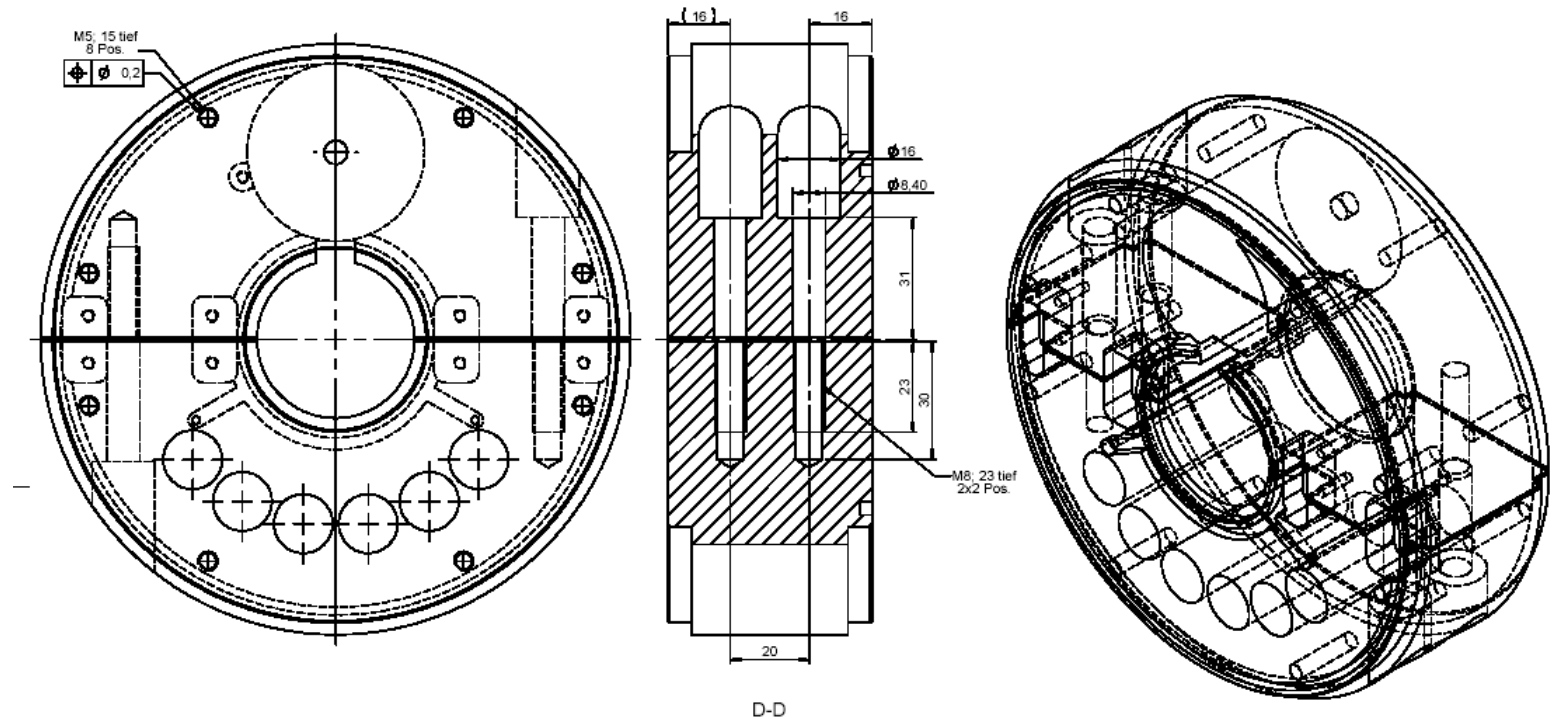

Abbildung 4.11: Senderträger

Die Batterieverkabelung und die Sendeantenne sind im Träger integriert. Um eine ausreichende Sendeleistung zu gewährleisten, muss die Versorgungsspannung des Sendemoduls zwischen 5,6 und 9,0V DC betragen. An das Sendemodul dt208/301 DC werden die Drucksensoren und die Sendeantenne angeschlossen. Das Modul dient der Konditionierung von Sensorsignalen und zur drahtlosen Übertragung der Messdaten von der rotierenden Rührerwelle zum stationären Telemetrie-Empfänger.

Der Messbereich der einzelnen Kanäle wird durch Verstärkungswiderstände definiert, die sich auf dem Anschlussfeld des Sendemoduls befinden. Der Sender ist vorkalibriert und der Messbereich beträgt für alle Kanäle $+/-5 m V$.

Der Empfänger $d t 282$ erfasst und reproduziert die Messdaten, welche vom TelemetrieSender über das Antennensystem übermittelt werden. Auf der Frontplatte befinden sich die Bedien- und Anzeigeelemente. Die Ein- und Ausgänge sind auf der Rückseite angeordnet. Die Ausgangsspannung am Empfänger beträgt für alle Kanäle 0 bis $+/-10 \mathrm{~V}$. 
Das Antennensystem dient der berührungslosen Messdatenübertragung vom Sender zum Empfänger. Es besteht aus einer Sendeantenne, die sich im Senderträger befindet, und aus einer stationären Empfangsantenne, die über ein Antennenkabel mit dem Empfänger verbunden ist.

\section{Funktionsprinzip}

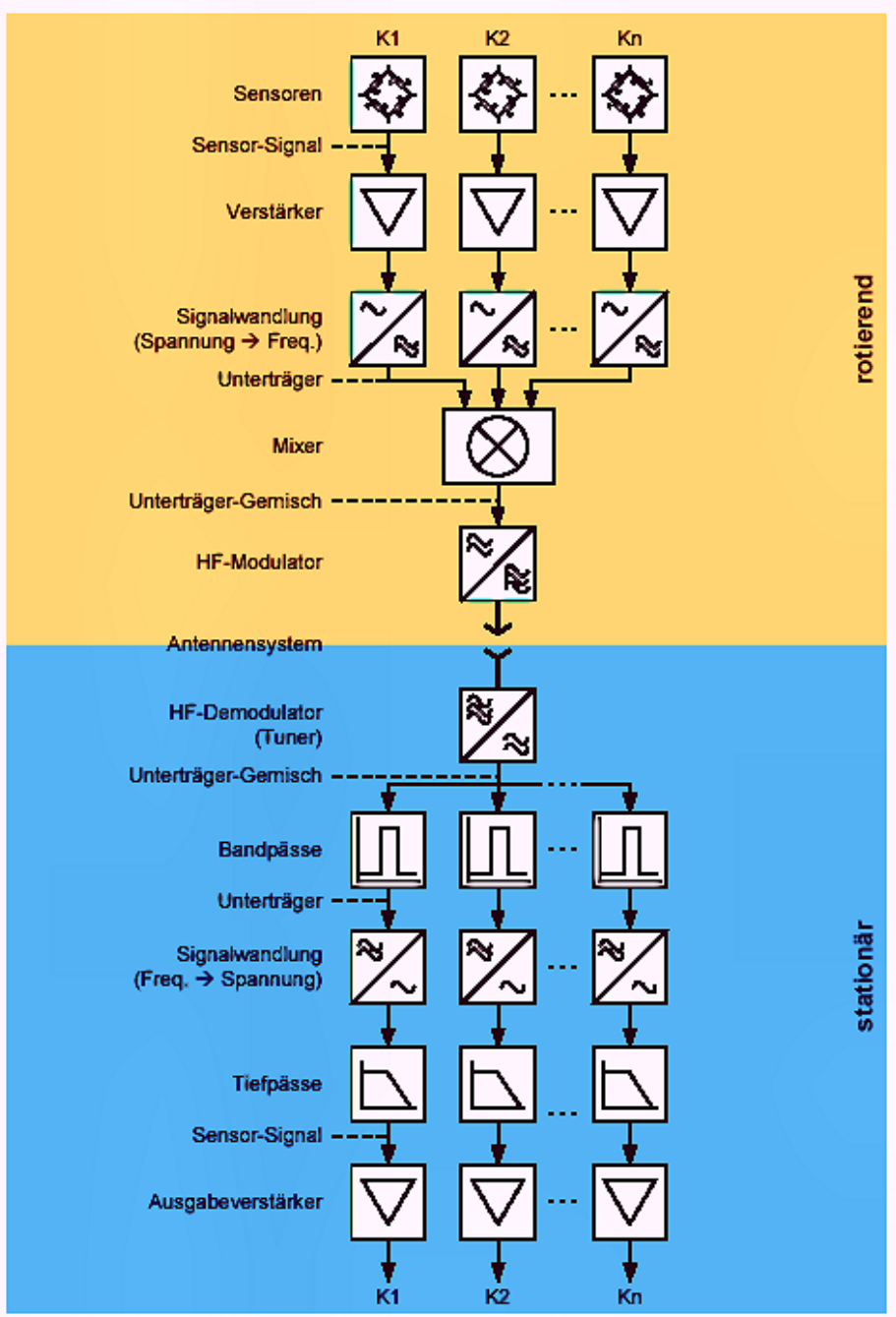

Abbildung 4.12: Funktionsprinzip des Telemetriesystems

Durch Dehnung oder Stauchung der Dehnmessstreifen (DMS) am Druckaufnehmer tritt eine Signaländerung am Verstärker ein. Die Sensorsignale werden mittels der Kabel zum Senderträger weitergeleitet, über den darin befindlichen Verstärker verstärkt und auf einen Normpegel konditioniert. Die Verstärkungen (= Messbereiche) werden über Widerstände auf dem Sender-Anschlussfeld eingestellt. Ein sogenannter „Mixer“ vereint die kanalspezifischen Unterfrequenzen. Die eingehende Spannung wird über eine Modulation in die Unterträgerfrequenz umgewandelt. Diese wird dann 
im HF-Modulator in die Hauptträgerfrequenz (HF-Signal) moduliert und von der Sendeantenne, die sich im Senderträger befindet, zur Empfangsantenne übertragen. Am Empfänger wandelt der HF-Demodulator das Signal wieder in die Unterträgerfrequenz um (Demodulation). Sogenannte Bandpässe filtern die kanalspezifischen Unterträger aus dem Unterträgerfrequenz-Gemisch. Über eine zweite Demodulation wird nun die Unterträgerfrequenz wieder in eine Spannung umgewandelt und somit das Sensorsignal des jeweiligen Kanals reproduziert. Tiefpässe bereiten nun die reproduzierten Signale auf. Sie werden am Ausgabeverstärker analog verstärkt und über die Ausgänge des Empfängers an das Spider 8-Messdatenerfassungssystem weitergegeben.

Zur winkelaufgelösten Auswertung, wie z.B. zur Veranschaulichung des Einflusses der Strombrecher auf die Druckverläufe, wird eine Datentriggerbox oberhalb des Rührguts vor einem Strombrecher eingesetzt. Der von der Triggerbox ausgehende Laserstrahl, der auf die Welle gerichtet ist, misst die Intensität des reflektierten Strahls und gibt diese Information als Signal weiter. In Richtung des Schwerpunkts wird auf der Welle auf Höhe des Datentriggers ein schmaler Klebestreifen appliziert, so dass vom Datentrigger jedes Mal ein Signal ausgegeben wird, wenn der Schwerpunkt diesen Strombrecher passiert.

\section{Versuchsdurchführung}

\section{Kalibrierung (Skalierung)}

Die Einstellung des gewünschten Messbereichs erfolgt durch Änderung der Verstärkungswiderstände $R$ im Sendemodul. Durch Einengung des Messbereichs auf \pm 300 mbar wird die Empfindlichkeit des Druckaufnehmers erhöht, da das unerwünschte Rauschen relativ zur Größe des Messsignals abnimmt.

Den Ausgangsspannungen der einzelnen Kanäle muss nach der Demodulation der entsprechende Druck zugeordnet werden. Da die Druckaufnehmer unterschiedliche Ein- und Ausgangsimpendanzen haben, geschieht das für jeden Kanal separat.

Hierzu werden der Rührerkopf und damit auch die Druckaufnehmer zunächst genau unterhalb der Wasseroberfläche positioniert. In dieser Stellung wird der Druck mittels der Spider 8-Software am Auswertecomputer tariert. Anschließend wird der Rührer genau um $10 \mathrm{~cm}$ im Wasser abgesenkt. Die vom Messdatenerfassungssystem Spider 8 pro Messkanal angezeigte Spannung entspricht einem Druck von 10mbar. Diese Vorgehensweise der Kalibierung wird öfters wiederholt, um eventuell auftretende 
Ausreißer auszuschließen und pro Druckaufnehmer einen aussagekräftigen Kalibrierungsmittelwert bilden zu können. Anschließend werden die für die einzelnen Druckaufnehmer gefundenen Skalierungswerte für Wassertiefen von $20 \mathrm{~cm}$ und $30 \mathrm{~cm}$ überprüft.

Nachdem der Rührer auf Betriebshöhe abgesenkt und befestigt ist, wird der in dieser Wassertiefe herrschende Druck tariert. Der Druck bei Stillstand des Rührers im Einbauzustand beschreibt den Bezugsdruck für die Messungen im Betrieb.

\section{Messungen}

Zur Ermittlung der zeitabhängigen Fluidkräfte auf das Rührorgan werden Untersuchungen im Drehzahlbereich von $100 \mathrm{U} / \mathrm{min}$ bis $400 \mathrm{U} / \mathrm{min}$ in Steigerungsschritten von 10U/min durchgeführt. Die Abtastrate der Messungen beträgt $1200 \mathrm{~Hz}$ mit einem Besselfilter von $10 \mathrm{~Hz}$. Pro Messreihe und Druckaufnehmer werden 14400 Messpunkte aufgezeichnet. 


\subsection{Experimentelle Ergebnisse im Leerlauf (Luft)}

In diesem Kapitel werden zunächst nur die experimentellen Ergebnisse dargestellt, welche als Randbedingung für das numerische Modell benötigt werden. Hierzu gehören:

- Materialdämpfungskonstante $\varsigma$

- $\quad$ Exzentrizität $\varepsilon$

Die weiteren experimentellen Ergebnisse werden in Kapitel 5 im Vergleich mit den numerischen Untersuchungen vorgestellt und bewertet. Sie dienen hauptsächlich der Validierung des numerischen Modells.

\subsubsection{Bestimmung der Eigenfrequenz und der Materialdämpfung}

Zur ersten einfachen Ermittlung der Eigenfrequenz und der Materialdämpfung der Rührerwelle mit den beiden zu untersuchenden Rührorganen werden zunächst Ausschwingversuche in Luft durchgeführt. Hierzu wird dem Rührwerk ein leichter Schlag versetzt; die Amplituden des Rührwerks werden über der Zeit aufgezeichnet. Es kann somit relativ einfach die Eigenfrequenz des Rührwerks in Luft bestimmt werden.

Durch Bildung des logarithmischen Dekrements $\Lambda$ des Betrags zweier aufeinanderfolgender Auslenkungsmaxima (siehe Gleichung 3.21) kann schließlich die Strukturdämpfungskonstante $\varsigma$ der Rührerwelle berechnet werden, welche als Randbedingung für die numerischen Simulationsrechnungen von Bedeutung ist (siehe Kapitel 3.2.4). Für das numerische Ersatzmodell, welches in Kapitel 5 mit den experimentellen Ergebnissen verglichen wird, ergibt sich ein Wert von $10 \mathrm{Ns} / \mathrm{m}$.

\subsubsection{Bestimmung der Exzentrizität}

Wie aus den Bewegungsgleichungen (3.28) ersichtlich, geht in die Amplitudenberechnung als äußere Last die Unwuchtkraft $F_{\text {Unwucht }}$ maßgeblich ein. Sie ist abhängig von der Exzentrizität $\varepsilon$, welche mit den Fertigungstoleranzen des Rührwerks zusammenhängt. Da diese Toleranzen für jedes Rührwerk verschieden sind, kann der Wert der Unwuchtkraft nicht rein rechnerisch ermittelt werden, sondern muss experimentell bestimmt werden. Nur so ist es möglich, das numerische Simulationsmodell und die experimentellen Versuchsergebnisse sinnvoll miteinander zu vergleichen. 
Im Leerlauf und für kleine Drehzahlen lässt sich folgendes statische Kräftegleichgewicht aufstellen:

$4 \cdot \pi^{2} \cdot n^{2} \cdot(x+\varepsilon) \cdot m=c \cdot\left(x-x_{0}\right)$

$x_{0}$ ist die geometrische Fehlstellung des Rührerkopfes bei Drehzahl Null, welche annähernd Null betragen wird.

$m$ beschreibt beispielhaft die reduzierte Masse des Ersatzsystems. Sie errechnet sich für den Schrägblattrührer zu 3,8428kg und für den $\mathrm{F} 1^{\odot}{ }^{-}$-Rührer $\mathrm{zu} 4,0895 \mathrm{~kg}$. Die Steifigkeit $c$ der Rührerwelle wird nach Gleichung (3.14) berechnet.

Die Auslenkungen können mit dem berührungslosen Messsystem für Rührwerksschwingungen im Betrieb bei unterschiedlichen Drehzahlen $n$ gemessen werden. Es werden eine Reihe von Auslenkungen $x_{i}$ bei unterschiedlichen Drehzahlen $n$, die alle ausreichend weit unterhalb der kritischen Drehzahl $n_{k r i t}$ liegen, aufgenommen und die RMS-Amplitude der Auslenkungen gebildet (siehe Abbildung 4.13). Die Messwertpaare, die sich indirekt proportional zum Quadrat verhalten, bilden die Grundlage für die Bestimmung der Exzentrizität $\varepsilon$.

Für die numerischen Ersatzmodelle der beiden Rührertypen ergeben sich folgende Werte:

Schrägblattrührer: $\quad \varepsilon=1,5 \mathrm{E}-04 m$

$F 1^{\odot}$-Rührer: $\quad \varepsilon=1,0 \mathrm{E}-04 m$

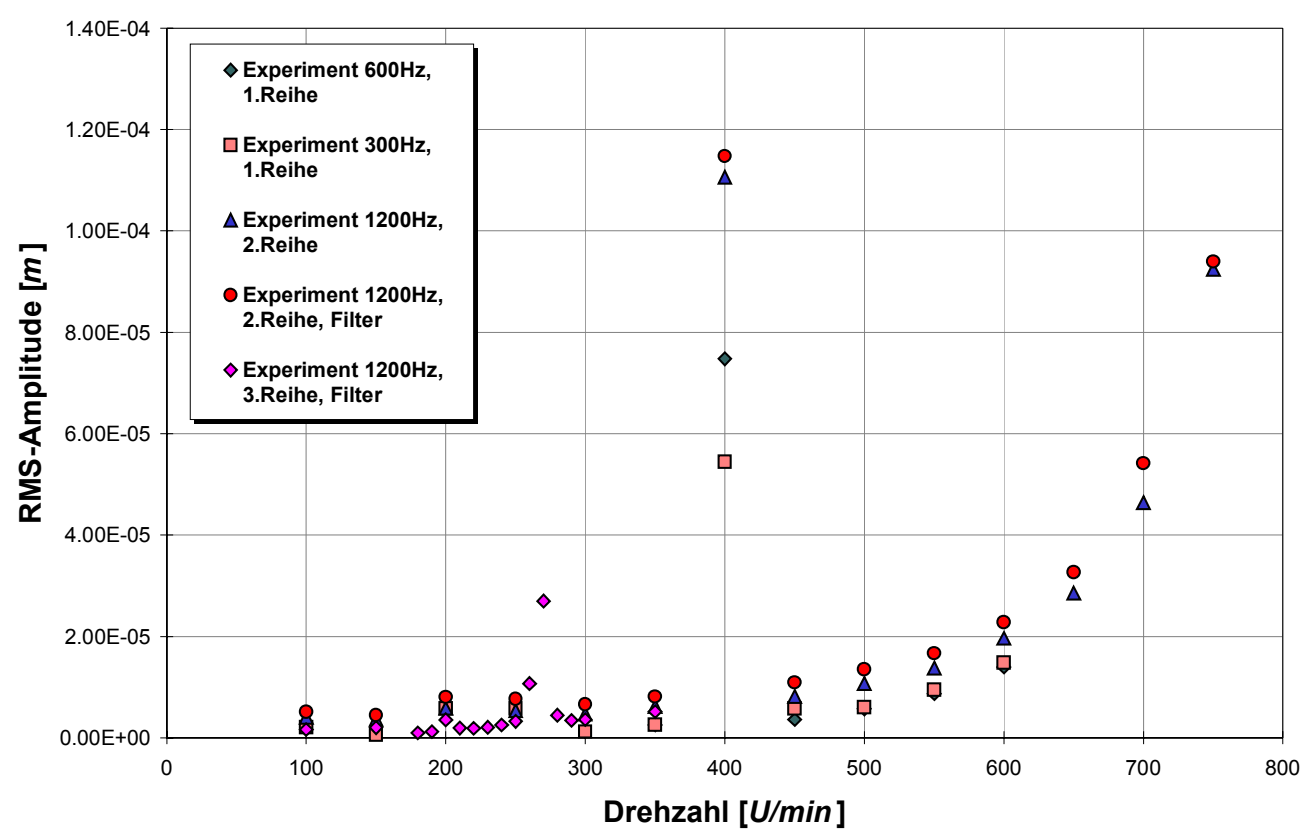

Abbildung 4.13: RMS-Amplituden beim Schrägblattrührer 
Die hohen RMS-Amplituden bei den Drehzahlen 270U/min und vor allem bei 400U/min resultieren aus dem Antriebsmotor, der bei diesen Drehzahlen erhebliche Schwingungen auf das Rührwerk überträgt. Durch bloßes Berühren des Antriebsmotors in diesen Drehzahlbereichen können diese Schwingungen festgestellt werden. Der Antriebsmotor verursacht in diesen Drehzahlbereichen auch erhebliche Geräuschentwicklungen, welche auf Eigenvibrationen zurückzuführen sind.

Die RMS-Amplituden in diesen Bereichen können somit nicht für die Bestimmung der Exzentrizität $\varepsilon$ verwendet werden. 


\section{Berechnungsbeispiele}

Es werden folgende Rührertypen numerisch und experimentell untersucht:

- Schrägblattrührer

- $\quad \mathrm{F} 1^{\odot}$-Rührer

Abbildung 5.1 und Abbildung 5.2 zeigen die numerischen Modelle der beiden untersuchten Rührertypen mit in einer Schnittebene dargestellten Strömungsfeldern bzw. Geschwindigkeitsvektoren.

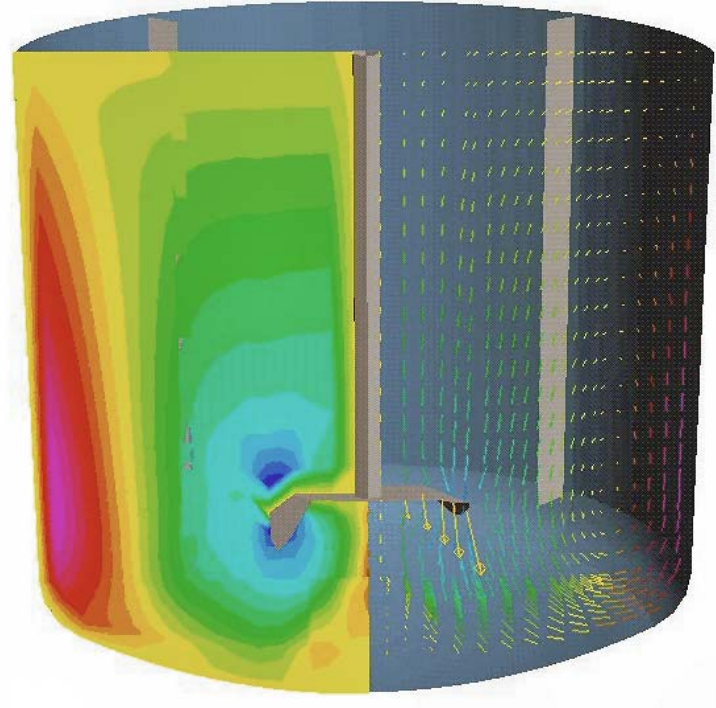

Abbildung 5.1: $F 1^{\odot}$-Rührer

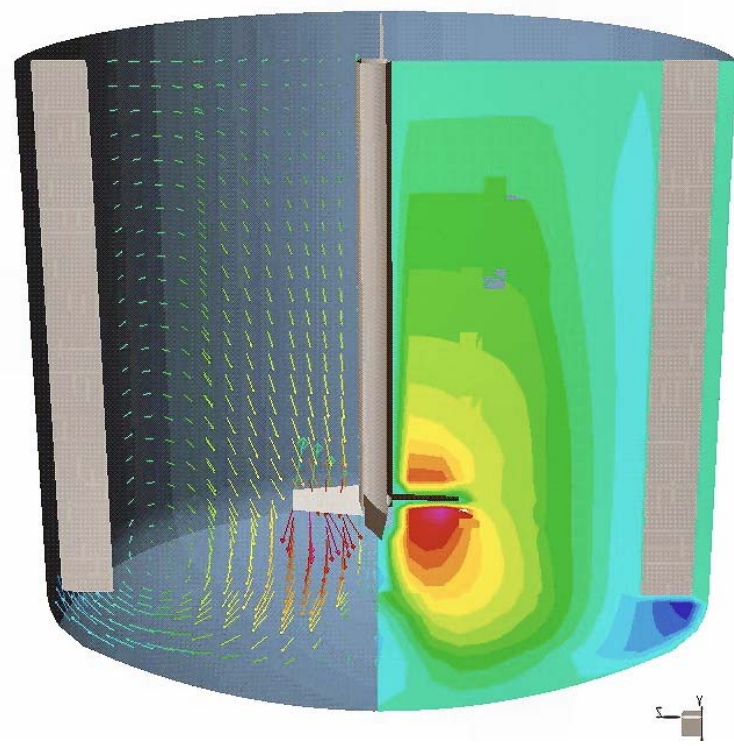

Abbildung 5.2: Schrägblattrührer

Die geometrischen Randbedingungen für die Simulationen ergeben sich aus den Vorgaben des Experiments und können Kapitel 4.1.1 entnommen werden.

\subsection{Vergleich des numerischen Ersatzmodells mit experimentellen Ergebnissen}

\subsubsection{Schrägblattrührer und $\mathrm{F1}^{\odot}{ }^{\circledR}$-Rührer im Leerlauf (Luft)}

Bei der Simulation im Leerlauf sind die numerischen Parameter Gitterfeinheit, Turbulenzmodellierung des Strömungsfelds bei den Drehzahlen unerheblich, da die Strömungskräfte als verschwindend klein zu betrachten sind und keinerlei Auswirkung auf das Strukturverhalten des Rührwerks haben. 
Der numerische Parameter „Zeitschrittweite“ wurde bereits in Kapitel 3.2.4 eingehend untersucht und für die folgenden Untersuchungen im Leerlauf mit 40TS/U festgelegt. Für sämtliche Simulationen im Leerlauf wird das zeitliche Diskretisierungsverfahren nach Newmark verwendet.

Das numerische Ersatzmodell des Schrägblattrührers wurde in Kapitel 3.2.4 mit FEMModellen, analytischen Modellen und dem numerischen Balkenmodell verglichen, und es zeigte sich eine sehr gute Übereinstimmung bezüglich der kritischen Drehzahl und den Auslenkungsamplituden.

Zur zusätzlichen Validierung des Frequenz- und des Amplitudenverhaltens von Schrägblattrührer und $\mathrm{F}^{\odot}{ }^{\odot}$-Rührer über verschiedenen Drehzahlen werden die numerischen Ersatzmodelle der beiden Rührertypen mit experimentellen Ergebnissen verglichen.

Ausschwingversuche sind die einfachste Möglichkeit, die Eigenfrequenz eines Rührwerks im Leerlauf experimentell zu bestimmen (siehe Kapitel 4.2.1).

Es ergeben sich hierbei folgende Ergebnisse für die kritische Drehzahl:

\begin{tabular}{||c|c|c||}
\hline \hline & Numerisches Modell & Ausschwingversuch \\
\hline Schrägblattrührer & $910 \mathrm{U} / \mathrm{min}$ & $915 \mathrm{U} / \mathrm{min}$ \\
\hline$F 1^{\odot}$-Rührer & $840 \mathrm{U} / \mathrm{min}$ & $850 \mathrm{U} / \mathrm{min}$ \\
\hline
\end{tabular}

Tabelle 5.1: Vergleich der kritischen Drehzahlen aus numerischem Ersatzmodell und Ausschwingversuchen

Die Ergebnisse der Ausschwingversuche, welche eine erste Vorabüberprüfung darstellen sollen, stimmen sehr gut mit den numerischen Simulationsergebnissen überein, liefern allerdings keinerlei Aussage über die RMS-Amplituden im Betrieb. 


\section{Schrägblattrührer im Leerlauf}

Abbildung 5.3 zeigt den RMS-Amplitudenverlauf des Schrägblattrührers über verschiedenen Drehzahlen im Leerlauf.

Die Größe der Strukturdämpfungskonstante wurde in den Simulationsläufen variiert, um ihren Einfluss auf das Schwingungsverhalten des Rührwerks aufzuzeigen. $\varsigma$ hat keinen feststellbaren Einfluss auf die kritische Drehzahl, wohl aber auf die RMSAmplitude, vor allem im Bereich der kritischen Drehzahl. Für den Fall $\varsigma=0$ (dämpfungsfrei) wurde der Simulationslauf bei $n_{k r i t}$ nach ca. 100 Umdrehungen abgebrochen, ohne dass sich der Rührer eingeschwungen hatte. Es ist zu erwarten, dass die Amplitude des Rührers bei längerer Simulationsdauer noch weiter ansteigt, bis der Simulationslauf divergiert.

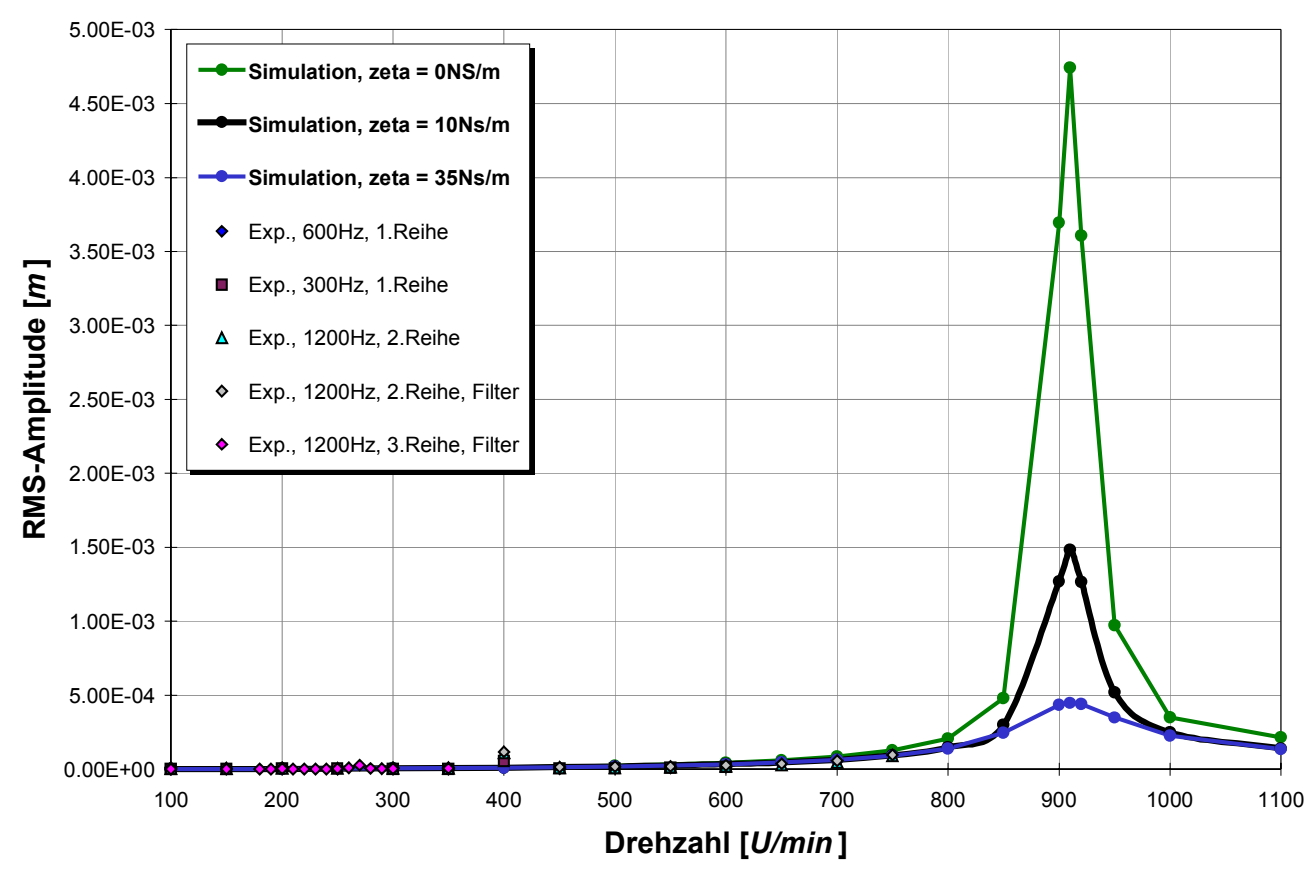

Abbildung 5.3: Schrägblattrührer im Leerlauf

Zur Veranschaulichung wird in Abbildung 5.4 der experimentell untersuchte Drehzahlbereich nochmals vergrößert dargestellt. 


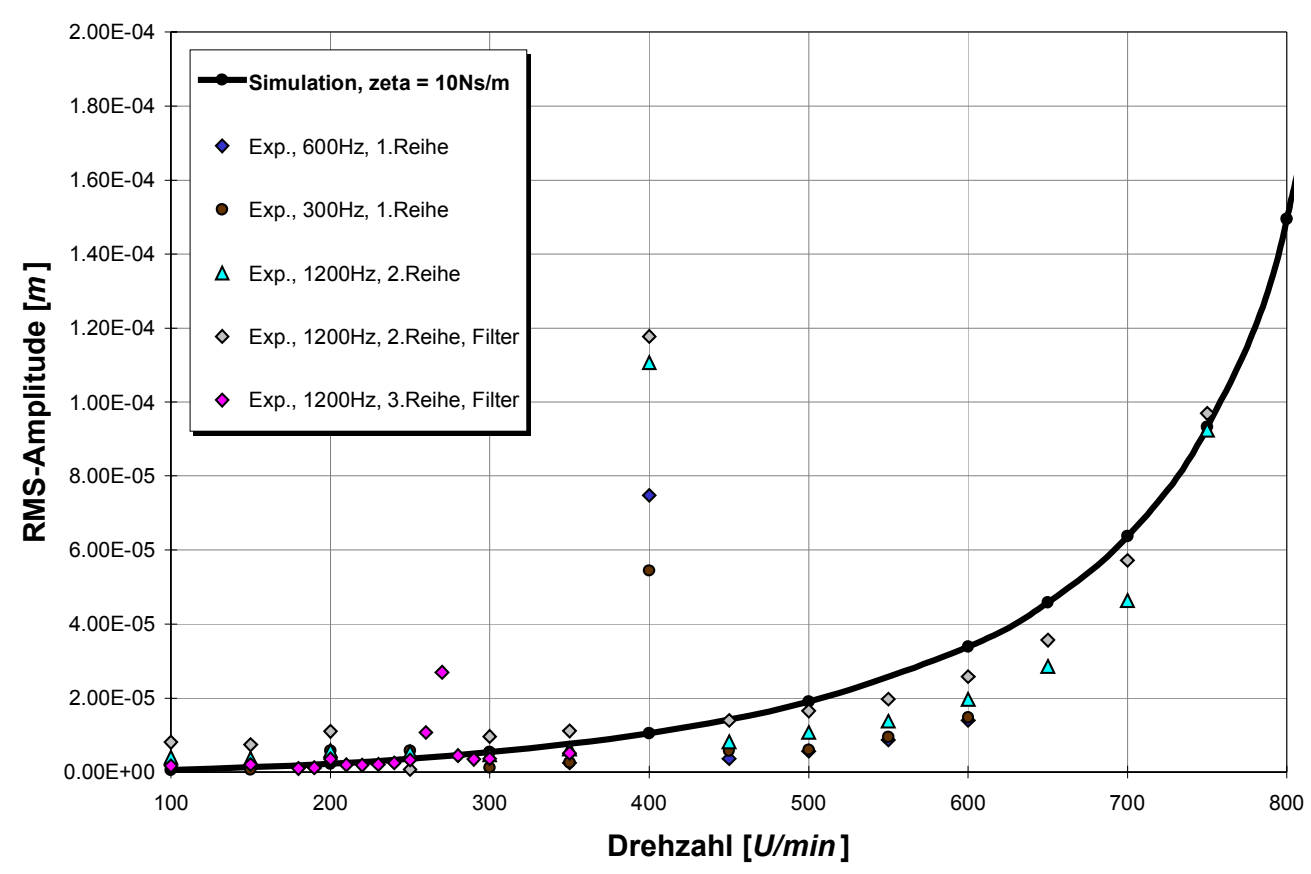

Abbildung 5.4: Vergleich Simulation - Experiment im Leerlauf

Es ist deutlich zu erkennen, dass das numerische Ersatzmodell mit $\varsigma=10 \mathrm{NS} / \mathrm{m}$ (aus Aussschwingversuch) und die experimentellen Ergebnisse eine sehr gute Übereinstimmung zeigen. Die Abweichungen in Drehzahlbereichen von ca. 400U/min resultieren aus den Vibrationen des Antriebsmotors.

Eine weitere Möglichkeit, die numerischen mit den experimentellen Ergebnissen zu vergleichen, ist die Untersuchung der Bewegungsbahn (Trajektorie) des Wellenmittelpunktes am Messpunkt.

In Abbildung 5.5 ist die Bewegungsbahn des Rührwerksmittelpunktes beispielhaft bei 700U/min dargestellt. Der Rührwerksmittelpunkt beschreibt bei ideal starrer Einspannung über den gesamten Drehzahlbereich eine Kreisbahn. Die Größe der Auslenkungsamplitude ist abhängig von der Drehzahl.

Die von Knoch [54] und Traupel [109] beschriebenen Phänomene, bei denen die Bewegungsbahn des Rührers bei Annäherung an die kritischen Drehzahlen die Form einer immer schmaler werdenden Ellipsenbahn annimmt, gilt nur für den Fall der elastischen Lagerung des Rührwerks. Ist das Rührwerk in zwei Richtungen unterschiedlich starr gelagert, treten eine Spaltung der kritischen Drehzahl und somit zwei Amplitudenmaxima auf. 
Im vorliegenden Fall ist das Rührwerk sowohl in der Simulation als auch im Experiment in alle Richtungen als starr eingespannt $\left(c_{E}=1,55 \cdot 10^{7} \mathrm{~N} / \mathrm{m}\right) \mathrm{zu}$ betrachten.

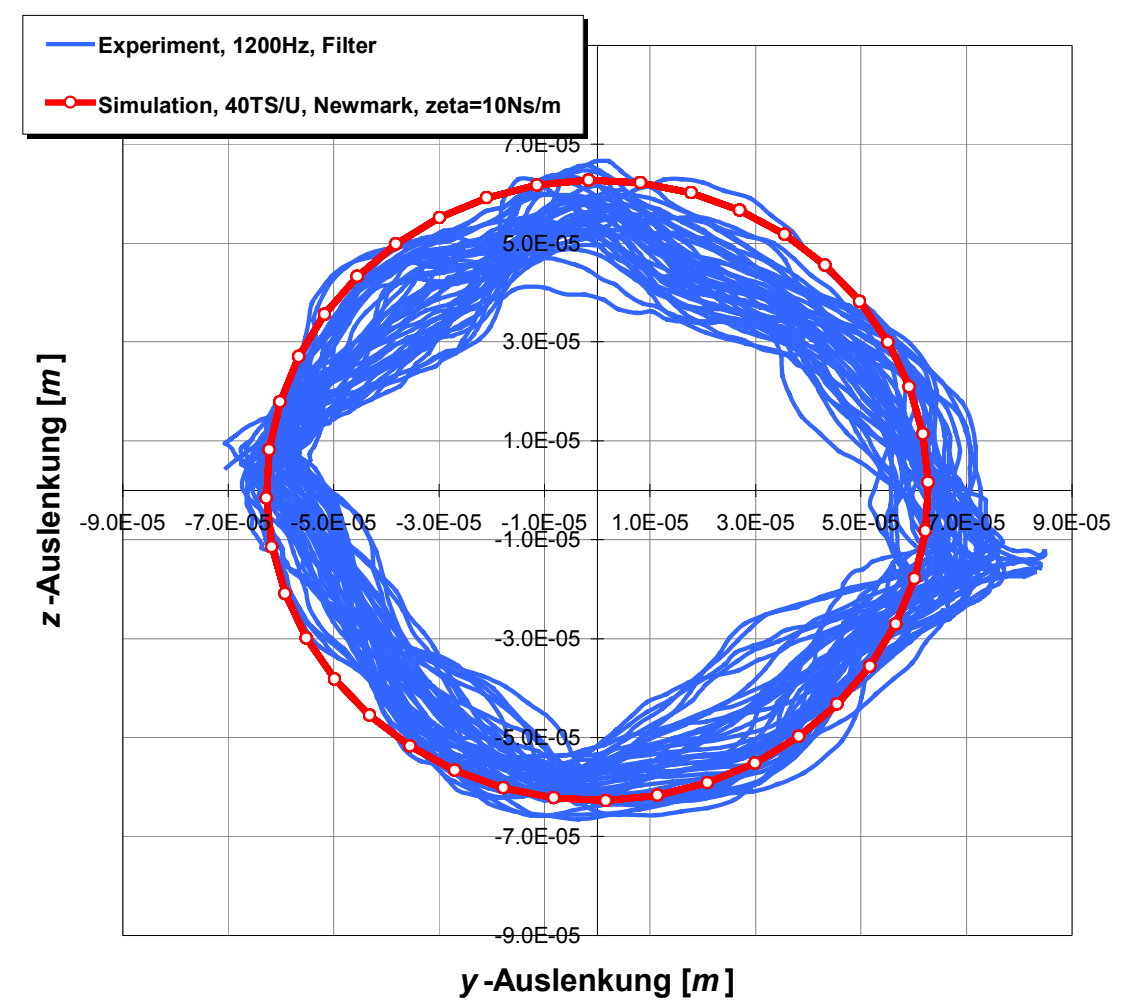

Abbildung 5.5: Trajektorie des Schrägblattrührers im Leerlauf bei 700U/min

Der unregelmäßige Verlauf der Trajektorie im Experiment ist, entsprechend der Ausführungen von Ziegler [120] auf Störeinflüsse durch die Lagerung der Rührwelle zurückzuführen. 


\section{F1 ${ }^{\circledR}$-Rührer im Leerlauf}

Zur Veranschaulichung des Exzentrizitätseinflusses auf das Amplitudenverhalten wird in Abbildung 5.6 exemplarisch die RMS-Amplitude des $\mathrm{F} 1^{\circledR}$-Rührers über der Drehzahl für verschiedene Exzentrizitäten aufgetragen.

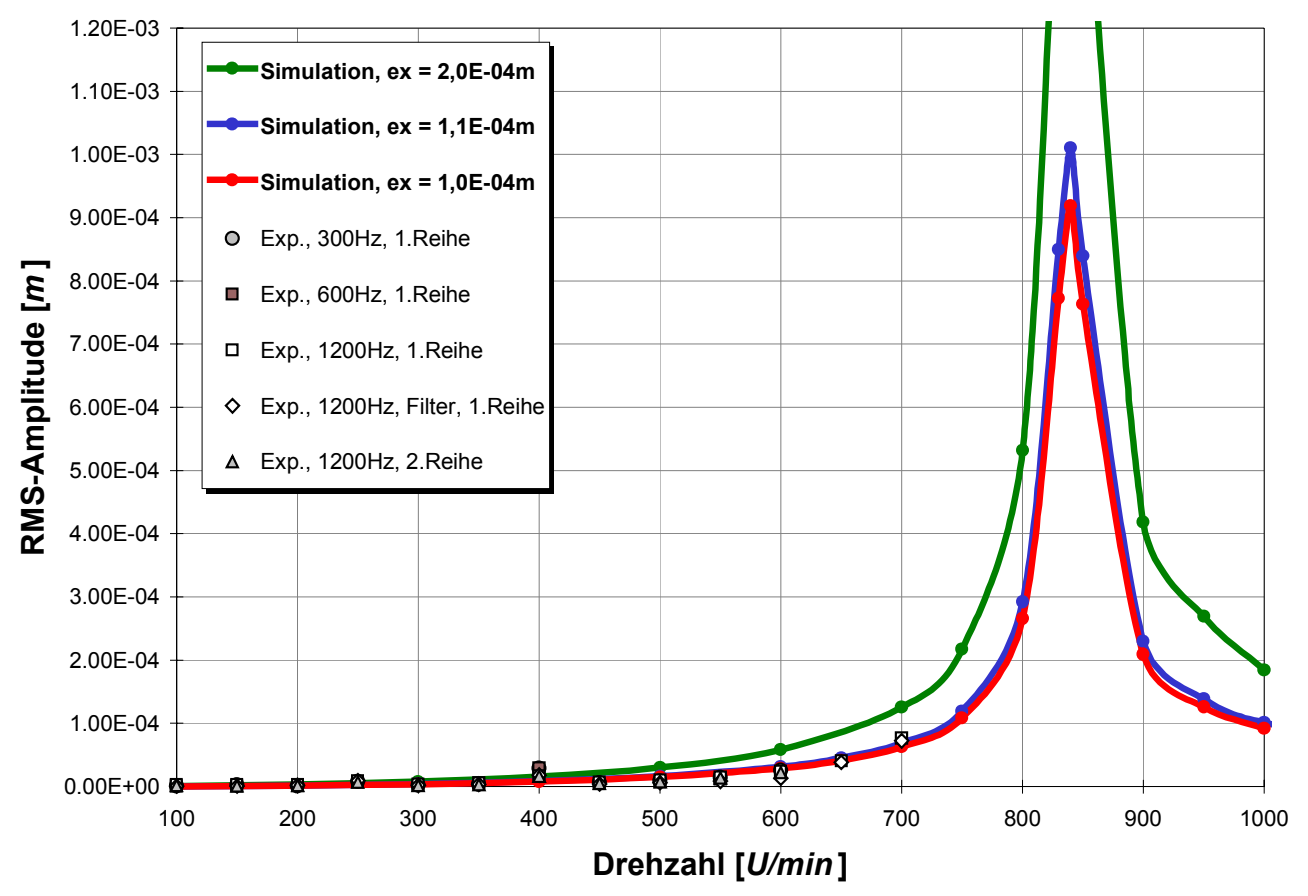

Abbildung 5.6: $F 1^{\odot}$-Rührer im Leerlauf

Wie zu erwarten, nimmt die RMS-Auslenkungsamplitude des Rührwerks mit steigender Exzentrizität $\varepsilon$ zu. Die Exzentrizität hat jedoch keinerlei Einfluss auf die kritische Drehzahl.

Zum Vergleich mit den experimentellen Untersuchungsergebnissen muss die Simulationskurve mit $\varepsilon=1,0 \mathrm{E}-04 m$ betrachtet werden, da diese dem realen Fall des untersuchten Rührwerks entspricht (siehe Abbildung 5.7).

In Abbildung 5.7 wird der gemessene Drehzahlbereich nochmals vergrößert dargestellt. 


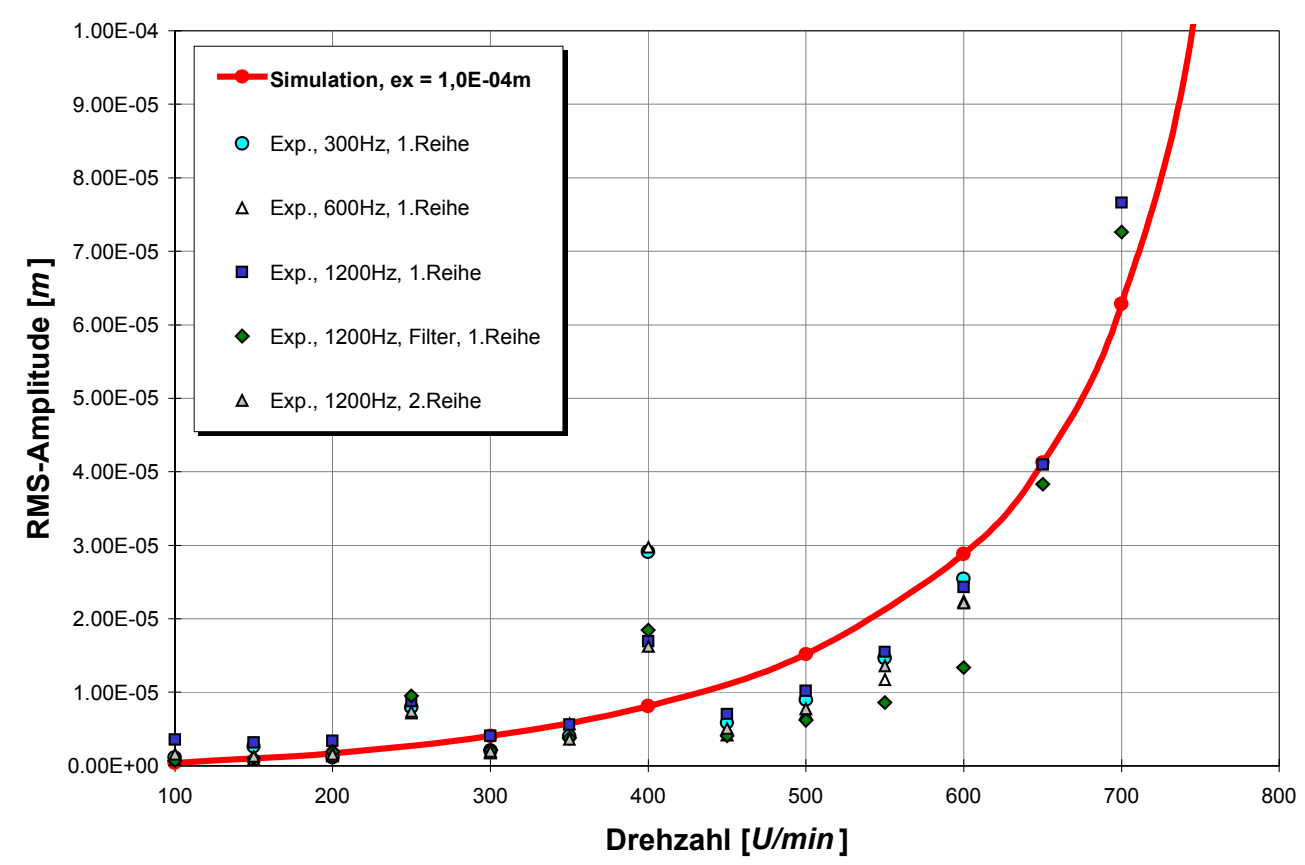

Abbildung 5.7: Vergleich Simulation - Experiment im Leerlauf $\left(F 1^{\odot}\right.$-Rührer)

Zusammenfassend lässt sich feststellen, dass die Simulationsergebnisse der numerischen Ersatzmodelle des Schrägblatt- und des F ${ }^{\odot}$-Rührers im Leerlauf sehr gut mit den experimentellen Untersuchungen übereinstimmen. 


\subsubsection{Schrägblattrührer und $F 1^{\odot}$-Rührer im Fluid (Wasser)}

\section{Validierung des numerischen Strömungsfelds}

Grundlage für eine richtige Simulation der Rührerbewegung (Strukturdynamik) und des Rührerfrequenzverhaltens im Fluid ist eine richtige numerische Lösung für das Strömungsfeld im Rührbehälter, welches in diesem Kapitel eingehend untersucht wird. Es werden folgende numerische Parameter gesetzt, die durch eigene Voruntersuchungen (siehe Kapitel 3.2.4) gefunden wurden:

- $\quad k-\varepsilon$ Turbulenzmodell

- Hybrid-Ansatz für die räumliche Diskretisierung des Strömungsfelds

- Zeitschrittweite 80TS/U

- 10 Iterationen pro Zeitschritt

Tabelle 5.2 und Tabelle 5.3 zeigen berechnete und gemessene Leistungseinträge der beiden Rührertypen bei einer Drehzahl von 600U/min. Der Leistungseintrag in der numerischen Strömungssimulation wird durch die Druckkräfte, die auf die Rührerblätter wirken, bestimmt:

$P=M \cdot \omega$

mit

$$
M=\frac{p}{A} \cdot y_{\text {Hebel }}
$$

\begin{tabular}{||c|c|c|c|c||}
\hline & $\begin{array}{c}\text { Simulation } \\
35000 \\
\text { Gitterzellen }\end{array}$ & $\begin{array}{c}\text { Simulation } \\
100000 \\
\text { Gitterzellen }\end{array}$ & Experiment & Differenz \\
\hline $600 U /$ min & $188,5 \mathrm{~W}$ & $188,4 \mathrm{~W}$ & $173,3 \mathrm{~W}$ & $8 \%$ \\
\hline
\end{tabular}

Tabelle 5.2: Vergleich des Leistungseintrags beim $F 1^{\circledR}$-Rührer 


\begin{tabular}{||l|c|c|c||}
\hline \hline & $\begin{array}{c}\text { Simulation } \\
35000 \\
\text { Gitterzellen }\end{array}$ & Experiment & Differenz \\
\hline $600 \mathrm{U} / \min$ & $305,2 \mathrm{~W}$ & $300,3 \mathrm{~W}$ & $2 \%$ \\
\hline
\end{tabular}

Tabelle 5.3: Vergleich des Leistungseintrags beim Schrägblattrührer

Die Ergebnisse bestätigen frühere Untersuchungen von Kohnen und Bohnet [56], nach denen sich der Leistungseintrag bei Berechnung aus Druckkräften kaum mit der Gitterfeinheit ändert. Der etwas zu hohe Leistungseintrag aus den Simulationen ist auf das k- $\varepsilon$ Turbulenzmodell zurückzuführen, welches die turbulente kinetische Energie leicht überschätzt wiedergibt [18], [31], [56].

Abbildung 5.8 und Abbildung 5.9 stellen die aus Simulation und LDA-Messung erhaltenen axialen Strömungsgeschwindigkeiten für die Systeme Schrägblattrührer bzw. $F 1^{\odot}$-Rührer gegenüber. Die Darstellung der axialen Geschwindigkeit über verschiedenen Winkelstellungen des Rührorgans gilt jeweils beispielhaft für den Punkt 4 (siehe Abbildung 4.6).

Die turbulenten Schwankungen sind im Experiment wesentlich stärker ausgeprägt als bei der Simulation mit dem $k$ - $\varepsilon$ Turbulenzmodell, welches diese Schwankungen herausdämpft. 


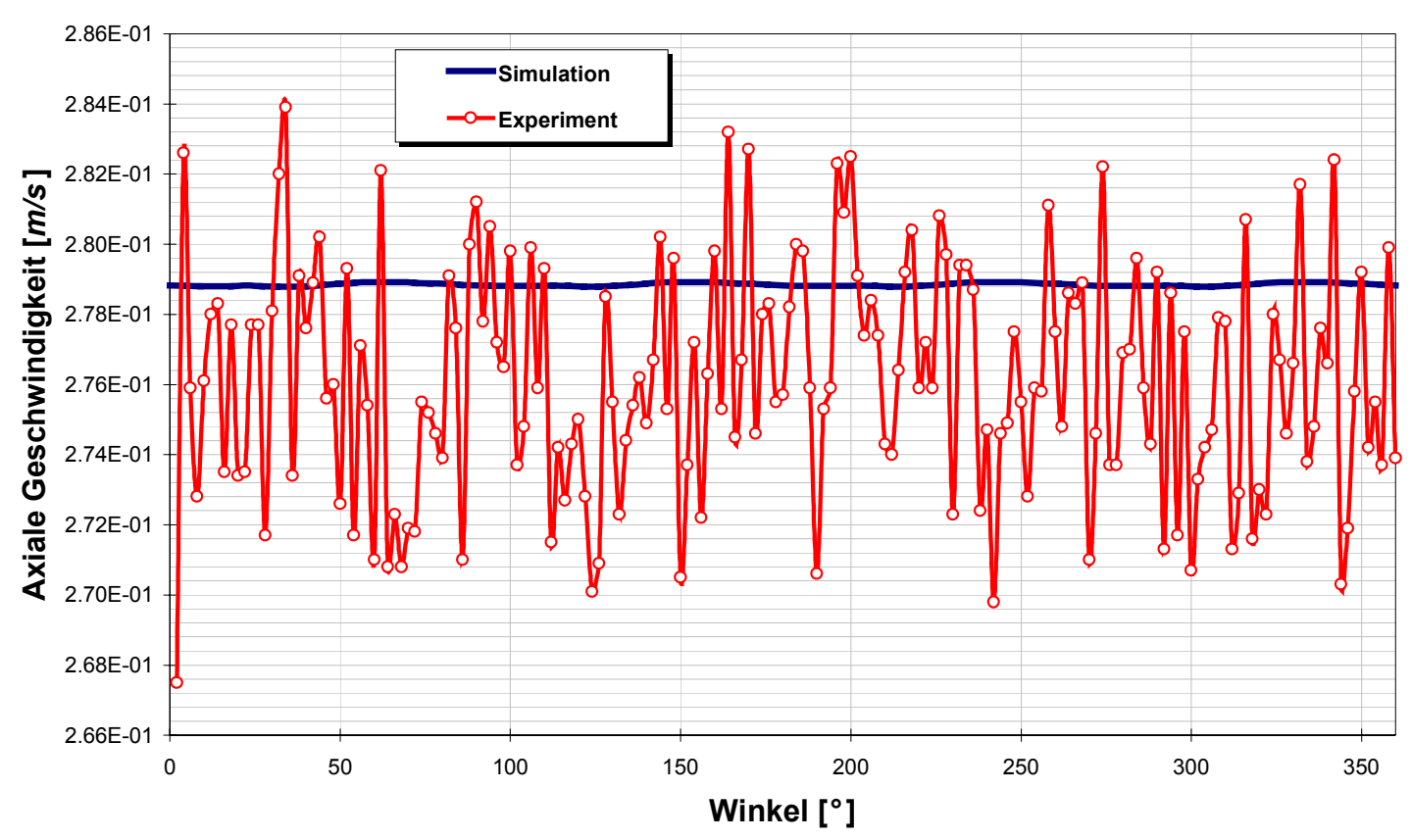

Abbildung 5.8: Vergleich der axialen Geschwindigkeit aus Simulation und Experiment beim Schrägblattrührer

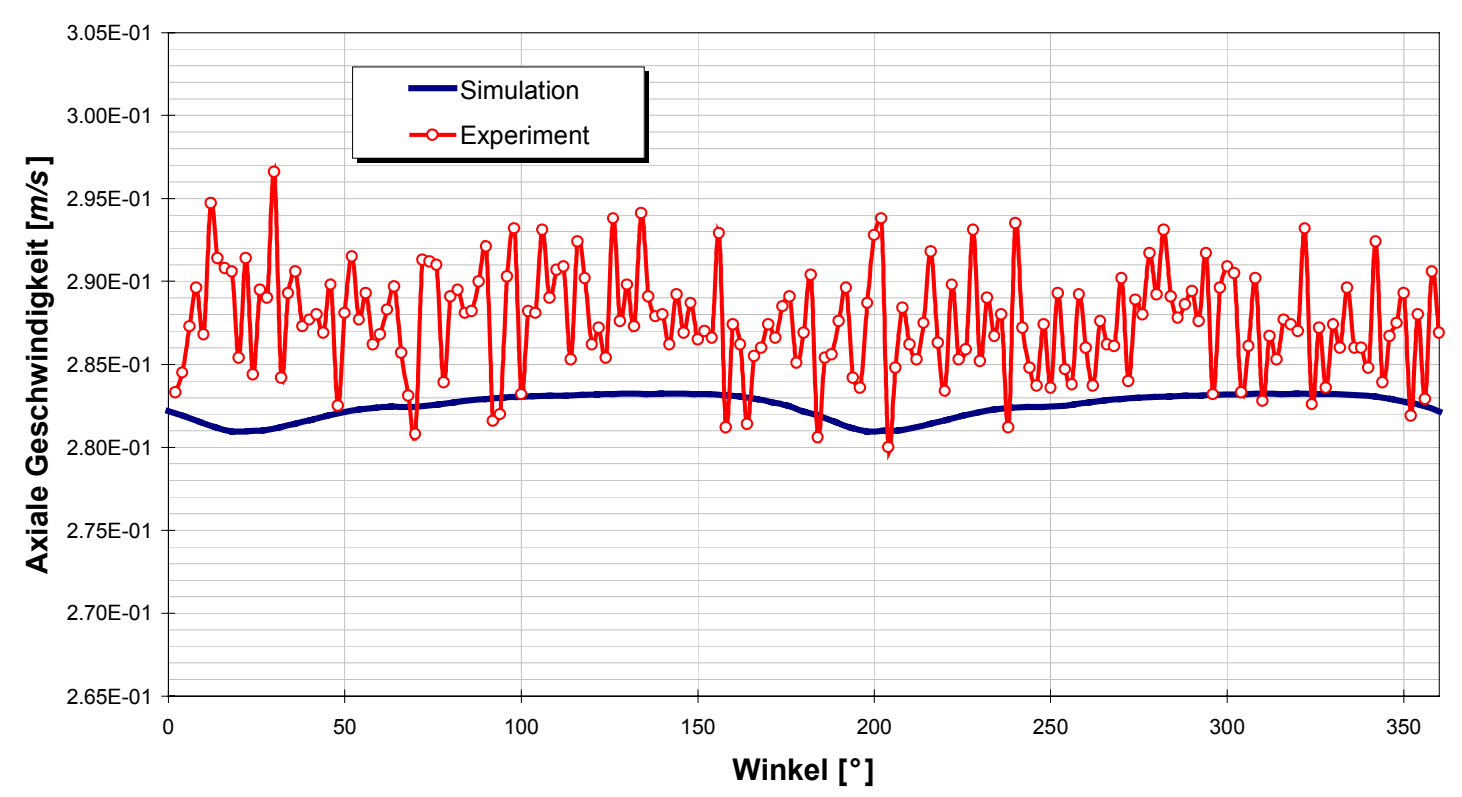

Abbildung 5.9: Vergleich der axialen Geschwindigkeit aus Simulation und Experiment beim $\mathrm{Fl}^{\circ}$-Rührer

In Abbildung 5.10 sind die gemittelten axialen Strömungsgeschwindigkeiten (Simulation und Experiment) an allen Messpunkten im Rührbehälter für beide Rührorgane zusammenfassend dargestellt. 


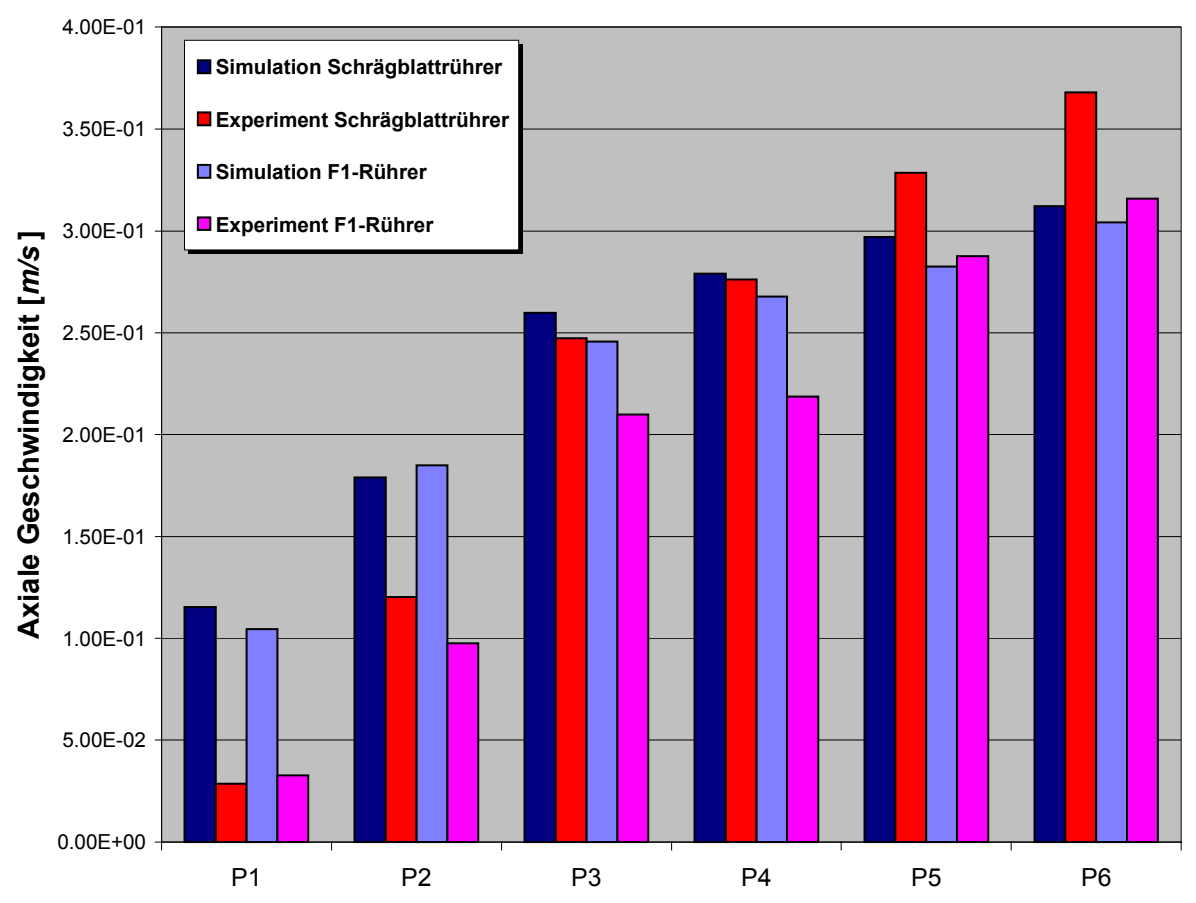

Abbildung 5.10: Vergleich der gemittelten axialen Strömungsgeschwindigkeiten aus Simulation und Experiment

Durch den Schrägblattrührer werden an den Messpunkten etwas größere axiale Strömungsgeschwindigkeiten erzeugt als beim $\mathrm{F}{ }^{\odot}$-Rührer. Die axiale Strömungsgeschwindigkeit nimmt erwartungsgemäß von der Rührmediumoberfläche (P1) zum Rührorgan (P6) zu.

Die relativ großen Unterschiede von Experiment und Simulation bei P1 und P2 bei beiden Rührwerken sind mitunter auf die nicht ganz übereinstimmende Lage der Monitorpunkte von Simulation und Experiment in diesem Bereich des Rührbehälters zurückzuführen. Außerdem wurde aufgrund der geringeren Geschwindigkeitsgradienten im oberen Bereich des Rührbehälters bei beiden Rührorganen ein gröberes Rechengitter modelliert, was einerseits zu Rechenzeitersparnissen, andererseits allerdings zu den beobachtbaren Rechenungenauigkeiten führen kann. Eine weitere Fehlerquelle sind Messungenauigkeiten, die durch Verunreinigungen des Rührmediums (zu schwere Tracerpartikel) hervorgerufen werden und sich vor allem in Bereichen geringerer Strömungsgeschwindigkeiten gravierender auswirken.

Zusammenfassend lässt sich feststellen, dass gerade in dem Strömungsbereich des Rührorgans, der mit einem feinerem Rechengitter aufgelöst wurde, eine sehr gute Übereinstimmung zwischen Simulation und Experiment vorherrscht. 


\section{Schrägblattrührer im Wasser}

Für den folgenden Vergleich zwischen Simulation und Experiment wurde das numerische Ersatzmodell des Schrägblattrührers mit einer experimentell bestimmten Exzentrizität $\varepsilon$ von 1,5E-04m, einer Gitterzellenanzahl von 35000 , dem $k-\varepsilon$ Turbulenzmodell und dem Newmark-Diskretisierungs-Verfahren mit einer Zeitschrittweite von $80 T S / U$ verwendet. Die Strukturdämpfungskonstante $\varsigma$ wurde zur Veranschaulichung ihres Einflusses variiert.

Abbildung 5.11 zeigt den RMS-Amplitudenverlauf des Schrägblattrührers über verschiedenen Drehzahlen im Rührmedium Wasser. Die Strukturdämpfungskonstante $\varsigma$ wurde variiert.

Im Vergleich zum Leerlauf (siehe Abbildung 5.3), hat die Strukturdämpfung im Wasser nur einen geringen Einfluss auf die RMS-Amplituden des Rührwerks, da die äußere Dämpfung durch das Rührmedium gegenüber der inneren Strukturdämpfung überwiegt [37], [49].

Für den Vergleich mit den experimentell ermittelten Werten muss die Kurve mit $\varsigma=10 N s / m$ herangezogen werden.

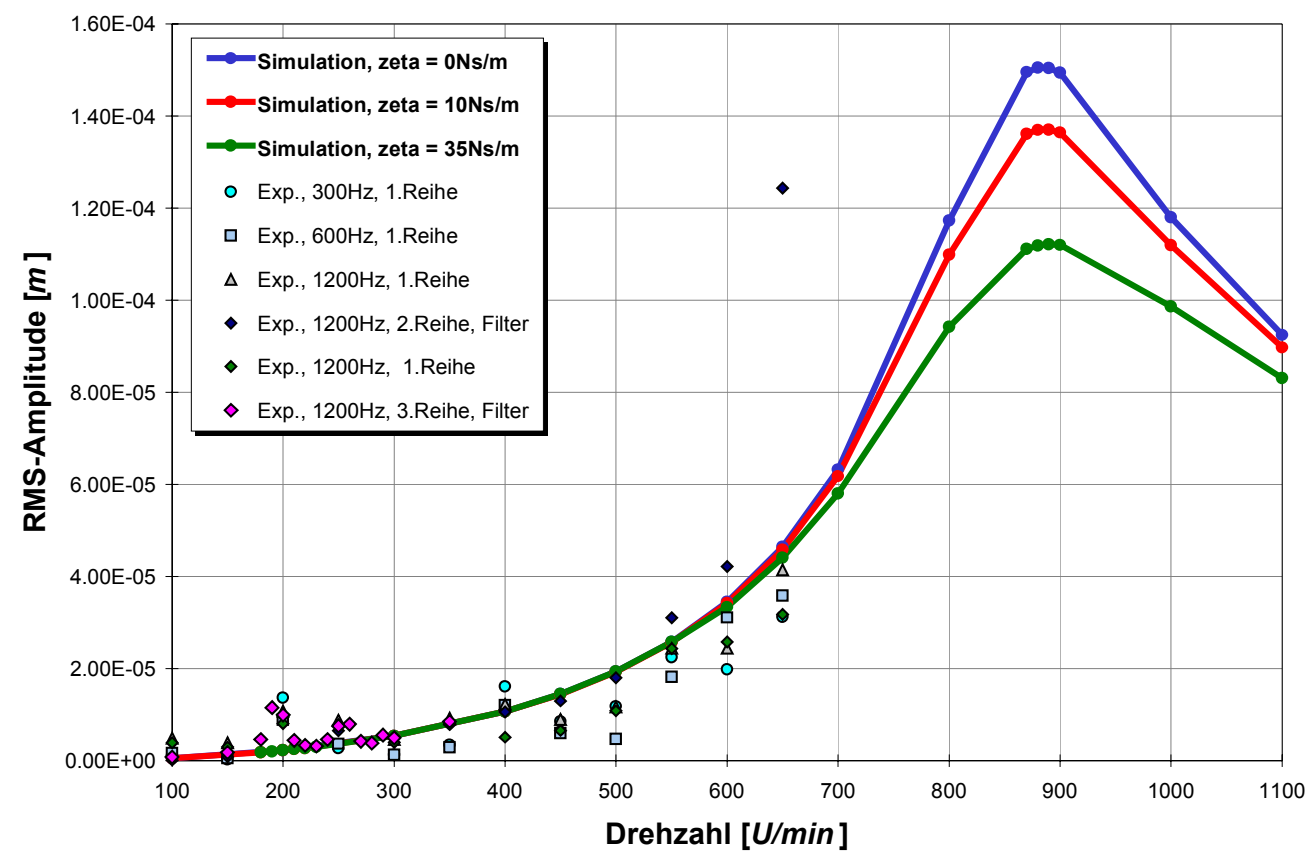

Abbildung 5.11: Vergleich Simulation - Experiment in Wasser (Schrägblattrührer)

Die Vibrationen des Antriebsmotors bei 400U/min, welche bei den Untersuchungen im Leerlauf noch deutlich zu erkennen waren, werden durch den Einfluss des Rührmediums Wasser stark gedämpft. 
Die Unterschiede zwischen Simulation und Experiment bei ca. 200U/min (unterkritischer Peak) resultieren aus einer fluidinduzierten Anregung, die mit dem Standard $k-\varepsilon$ Turbulenzmodell nicht ausreichend genau wiedergegeben werden kann. Hierzu sind Simulationen mit Low-Re-Modellen (siehe Abbildung 3.14) nötig, welche ein Vielfaches an Gitterzellen und somit auch an Speicherplatz und Rechenzeit benötigen.

\section{F1 ${ }^{\circledR}$-Rührer im Wasser}

Für den folgenden Vergleich zwischen Simulation und Experiment wurde das numerische Ersatzmodell des $\mathrm{F}^{\odot}{ }^{\odot}$-Rührers mit einer experimentell bestimmten Exzentrizität $\varepsilon$ von $1,0 \mathrm{E}-04 m$, einer Gitterzellenanzahl von 35000 , dem $k$ - $\varepsilon$ Turbulenzmodell und dem Newmark-Diskretisierungs-Verfahren mit einer Zeitschrittweite von 80TS/U verwendet.

Abbildung $\mathbf{5 . 1 2}$ zeigt den RMS-Amplitudenverlauf des $\mathrm{F} 1^{\odot}$-Rührers über verschiedenen Drehzahlen im Rührmedium Wasser.

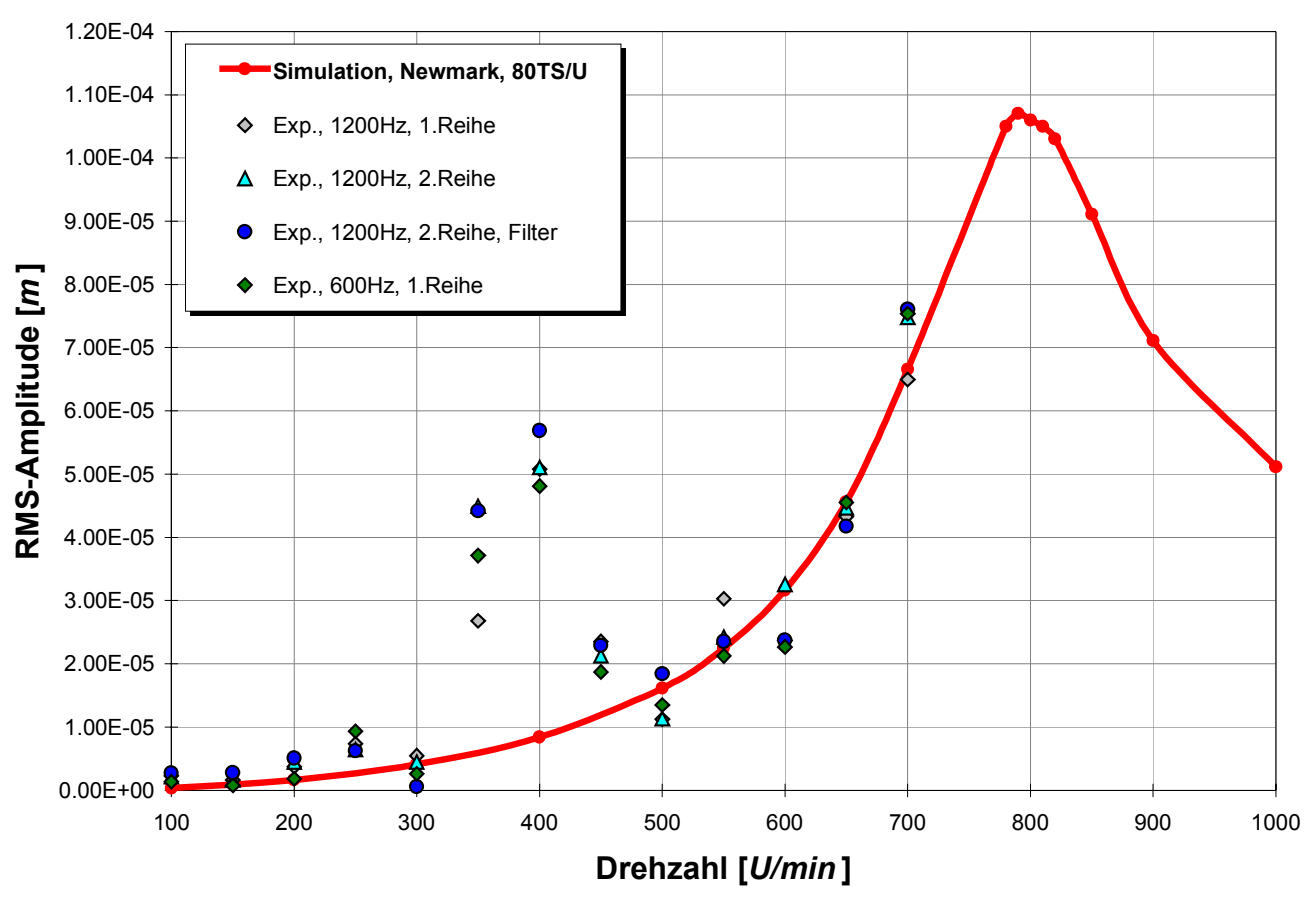

Abbildung 5.12: Vergleich Simulation - Experiment in Wasser $\left(F 1^{\circledR}\right.$-Rührer $)$

Der Vergleich zwischen Simulation und Experiment zeigt, dass vor allem im unterkritischen Drehzahlbereich bei ca. 400U/min Abweichungen zwischen Simulation und Experiment auftreten. Diese Abweichungen resultieren zum kleinen Teil aus den 
Vibrationen des Antriebsmotors in diesem Drehzahlbereich, welche allerdings, wie bereits beim Schrägblattrührer in Wasser deutlich zu erkennen war, in Wasser stark gedämpft werden.

Dieses ausgeprägte Amplitudenmaximum bei 400U/min (Experiment) im Wasser ist somit nicht auf Motorvibrationen zurückzuführen, sondern vielmehr auf eine fluidinduzierte Anregung. Der daraus resultierende unterkritische Peak der Auslenkungsamplitude des Rührwerks tritt beim zweiblättrigen $\mathrm{F} 1^{\odot}$-Rührer bei $n_{\text {krit }} / 2$ $=780 \mathrm{U} / \mathrm{min} / 2=390 \mathrm{U} / \mathrm{min}$ auf. 


\subsection{Einfluss verschiedener Fluide auf das Frequenzverhalten des Rührwerks}

\subsubsection{Einfluss des Fluids auf die kritische Drehzahl und die RMS- Amplituden}

In Abbildung 5.13 ist der RMS-Verlauf des Schrägblattrührers am Rührerkopf im Leerlauf, für Wasser und für Glukosesirup ( $\rho=2000 \mathrm{~kg} / \mathrm{m}^{3}, \eta=0,1$ Pas $)$ dargestellt. Eine Erhöhung der Dichte und der Viskosität des Rührmediums hat zur Folge, dass sich die kritische Drehzahl und vor allem die RMS-Auslenkungen des Rührwerks erheblich verringern.

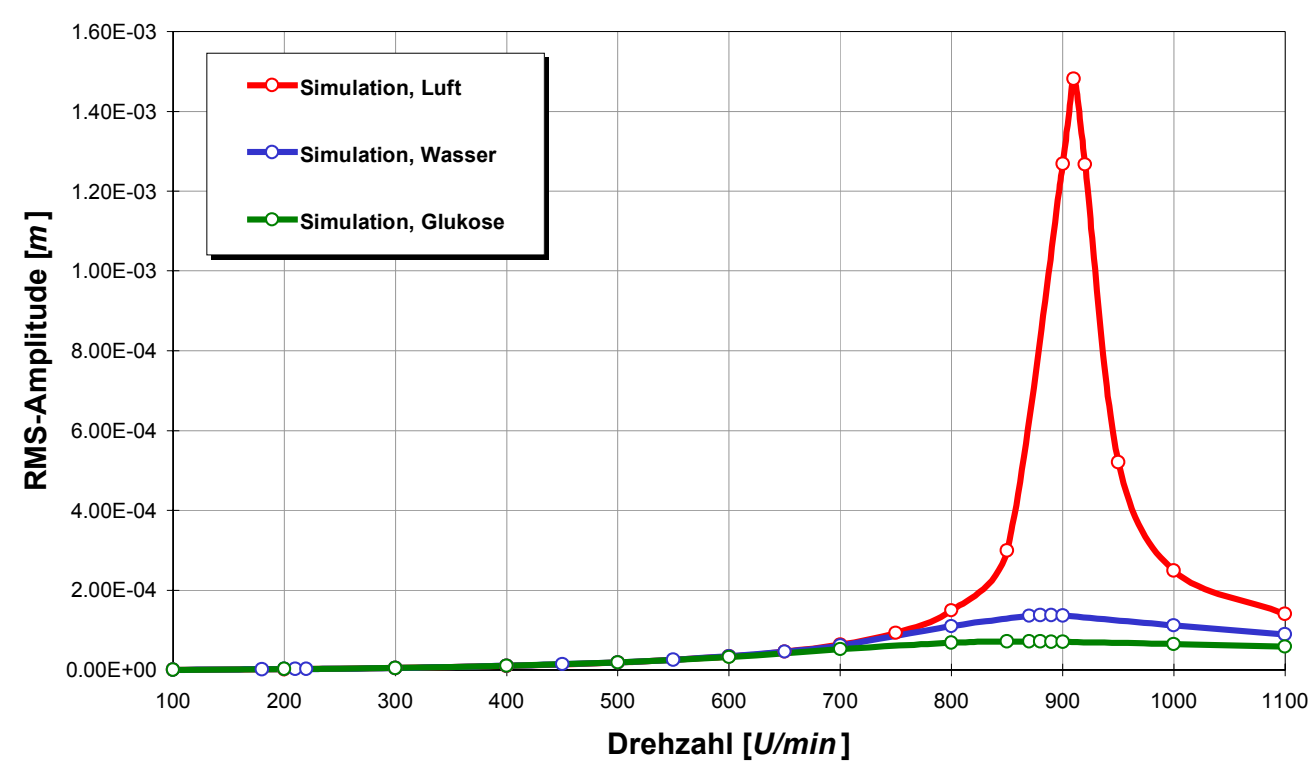

Abbildung 5.13: Einfluss des Fluids auf die kritische Drehzahl und die RMSAmplitude

In Tabelle 5.5 sind die kritischen Drehzahlen und die zugehörigen RMS-Amplituden am Rührerkopf für die drei Rührmedien nochmals zahlenmäßig zusammengefasst:

\begin{tabular}{|c|c|c|}
\hline & kritische Drehzahl & RMS-Amplitude \\
\hline Luft & $910 U / \mathrm{min}$ & $1,48 \mathrm{E}-03 \mathrm{~m}$ \\
\hline Wasser & $880 U / \mathrm{min}$ & $1,37 \mathrm{E}-04 \mathrm{~m}$ \\
\hline Glukosesirup & $870 U / \mathrm{min}$ & $7,14 \mathrm{E}-05 \mathrm{~m}$ \\
\hline
\end{tabular}

Tabelle 5.5: Kritische Drehzahlen und zugehörige RMS-Amplituden bei verschiedenen Fluiden 


\subsubsection{Fluidinduzierte Schwingung im unterkritischen Drehzahlbereich}

Stimmt die Frequenz der von der Rührbehälterströmung kommenden Impulse mit der Eigenfrequenz des Rührwerks überein, können auch im unterkritischen Bereich nicht ungefährliche Resonanzschwingungen auftreten. Diese sogenannte Blattdrehzahl ist beim vierblättrigen Schrägblattrührer als $n_{\text {Blatt }}=n_{\text {krit }} / 4$ definiert, was beim untersuchten Rührertyp etwa 200U/min entspricht. Durch eine Variation der Einbauten (Stromstörer) konnte deren Einfluss auf die RMS-Amplitude experimentell überprüft werden.

Jedes Mal, wenn ein Rührerblatt einen Stromstörer passiert, erhält es über das Rührmedium einen Impuls. Da bei vier gleichmäßig angeordneten Einbauten die jeweils gegenüberliegenden Blätter nahezu gleichzeitig diesen Strömungsimpuls erfahren, werden die Impulse kompensiert und wirken sich nicht so stark auf die RMSAmplitude des Rührwerks aus wie bei unsymmetrischen Einbauten (z.B. einem Stromstörer).

Es zeigt sich, dass ohne Einbauten kaum periodisch wiederholende Strömungsimpulse auf das Rührwerk wirken, welche dieses in Resonanzschwingungen versetzen könnten. Abbildung 5.14 stellt das Schwingungsverhalten des Schrägblattrührers im unterkritischen Drehzahlbereich dar.

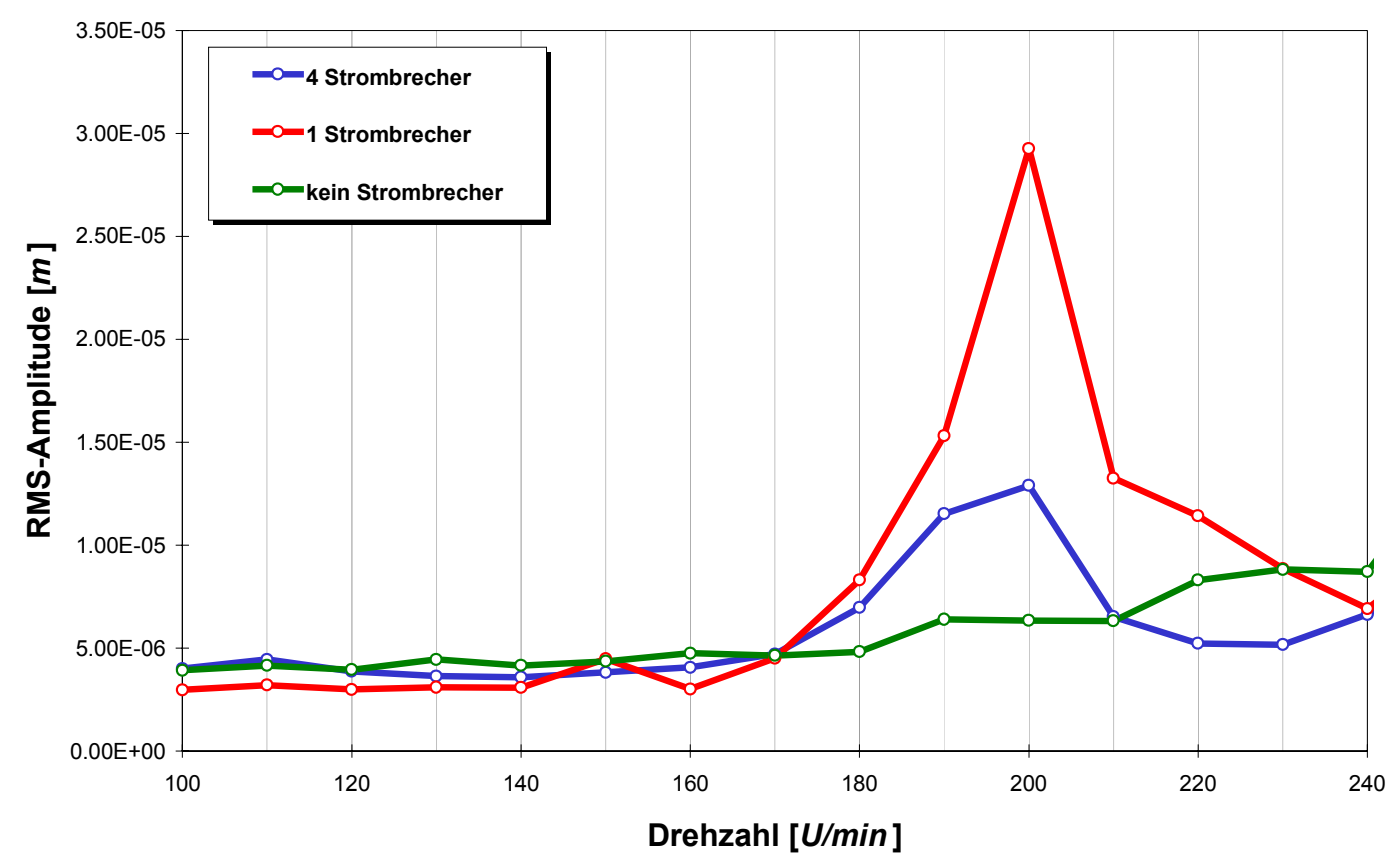

Abbildung 5.14: Unterkritischer Peak beim Schrägblattrührer 
Die relativ zum Stillstand des Rührwerks ermittelten Drücke auf die vier Rührerblätter des Schrägblattrührers können sowohl experimentell als auch numerisch an der Oberund Unterseite der Rührerblätter ermittelt werden.

In Abbildung 5.15 ist beispielhaft am Blatt 3 die Lage des Monitorpunkts für die folgenden Diagramme dargestellt.

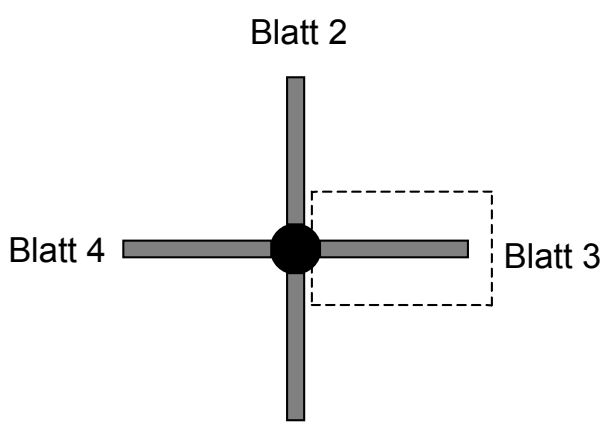

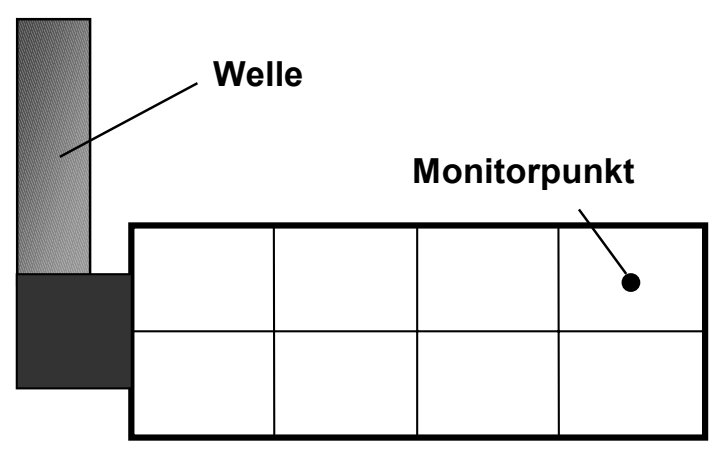

Blatt 3

Blatt 1

Abbildung 5.15: Lage des Monitorpunkts

In Abbildung 5.16 und Abbildung 5.17 werden die aus der Simulation ermittelten Drücke auf die vier Rührerblätter über der Zeit dargestellt. Im Rührbehälter befinden sich vier gleichmäßig verteilte Stromstörer $\left(90^{\circ}\right.$-Anordnung). Abbildung 5.16 zeigt den Druckverlauf für den Monitorpunkt (siehe Abbildung 5.15) an der Oberseite (Saugseite) des jeweiligen Blattes, Abbildung 5.17 an der Unterseite (Druckseite). Es wird eine Rührerumdrehung (80TS) bei 200U/min dargestellt.

Wie zu erwarten, ergeben sich auf der Oberseite der Rührerblätter relativ zum Stillstand betrachtet negative (Saugseite) und auf der Unterseite positive (Druckseite) Druckwerte. 


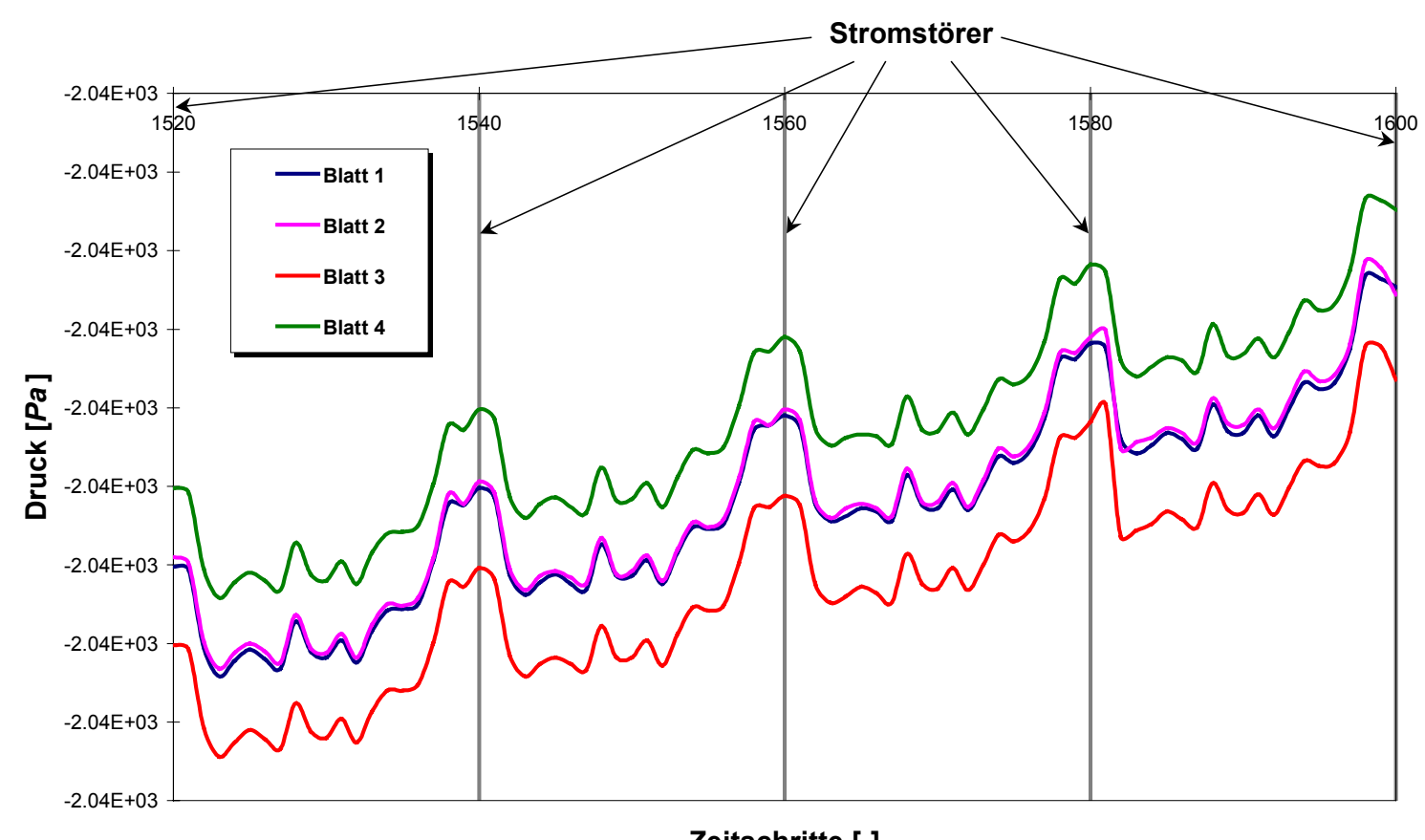

Zeitschritte [-]

Abbildung 5.16: Oberseite der Rührerblätter (4 Stromstörer)

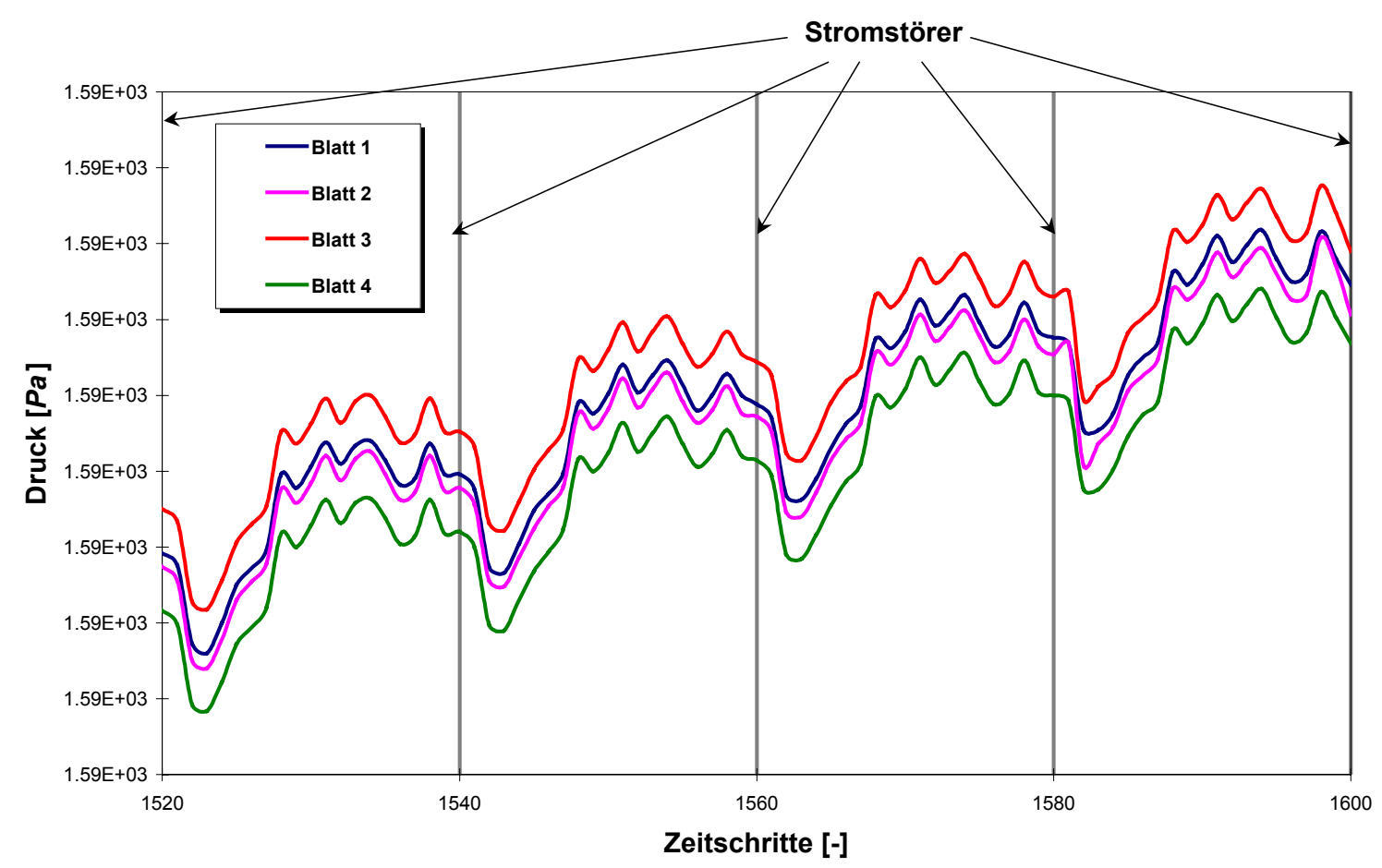

Abbildung 5.17: Unterseite der Rührerblätter (4 Stromstörer)

Die vier Rührerblätter passieren zeitgleich einen Stromstörer, was pro Rührerblatt zu vier Impulsen pro Umdrehung führt. Die jeweiligen Drücke auf Oberseite und Unterseite der einzelnen Rührerblätter sind nahezu identisch, so dass sich die Impulse 
auf gegenüberliegende Blätter kompensieren, und der daraus resultierende Gesamtströmungsimpuls auf das Rührwerk gering ist.

Der dennoch auftretende sehr geringe Druckunterschied auf die einzelnen Rührerblätter ist auf die unterschiedliche Auslenkung der Blätter zurückzuführen. Bei Blatt 3 ist der Schwerpunkt angenommen, so dass dieses Blatt im unterkritischen Drehzahlbereich am weitesten ausgelenkt wird und somit relativ zur Ruhelage den betragsmäßig größten Druck erfährt. Dementsprechend ist der Betrag des Drucks am gegenüberliegenden Blatt 4 am geringsten. Die Drücke auf Blatt 1 und Blatt 2 sind fast identisch, da diese Blätter auch die gleiche Kreisbahn beschreiben.

Es ist auffällig, dass auf Ober- und Unterseite kurz nach Passieren des Stromstörers der Druck auf ein Minimum absinkt, welches auf der Unterseite allerdings sofort wieder verlassen wird. Hier bildet sich eine Maximumebene aus. Auf der Oberseite der Rührerblätter bleibt der Druck nach Passieren des Stromstörers in einem Minimumtal, bis er kurz vor Passieren des Stromstörers wieder steil ansteigt, so dass beim Passieren des Stromstörers ein kurzzeitiges Druckmaximum vorliegt.

Ergänzend hierzu werden in Abbildung 5.18 die experimentellen Werte aus der Druckmessung an den beiden gegenüberliegenden Blättern Blatt 3 und Blatt 4 für den in Abbildung 5.15 dargestellten Monitorpunkt aufgezeigt. Auch hier ist zu erkennen, wie sich beim Passieren der Stromstörer die gleichen Drucktendenzen wie bei der Simulation ausbilden. Die Druckschwankungen auf die beiden Rührerblätter sind allerdings größer und nicht so regelmäßig wie bei der Simulation, was auf stochastisch auftretende Turbulenzen zurückzuführen ist, welche bei der Simulation mit dem $k$ - $\varepsilon$ Turbulenzmodell herausgedämpft werden. 


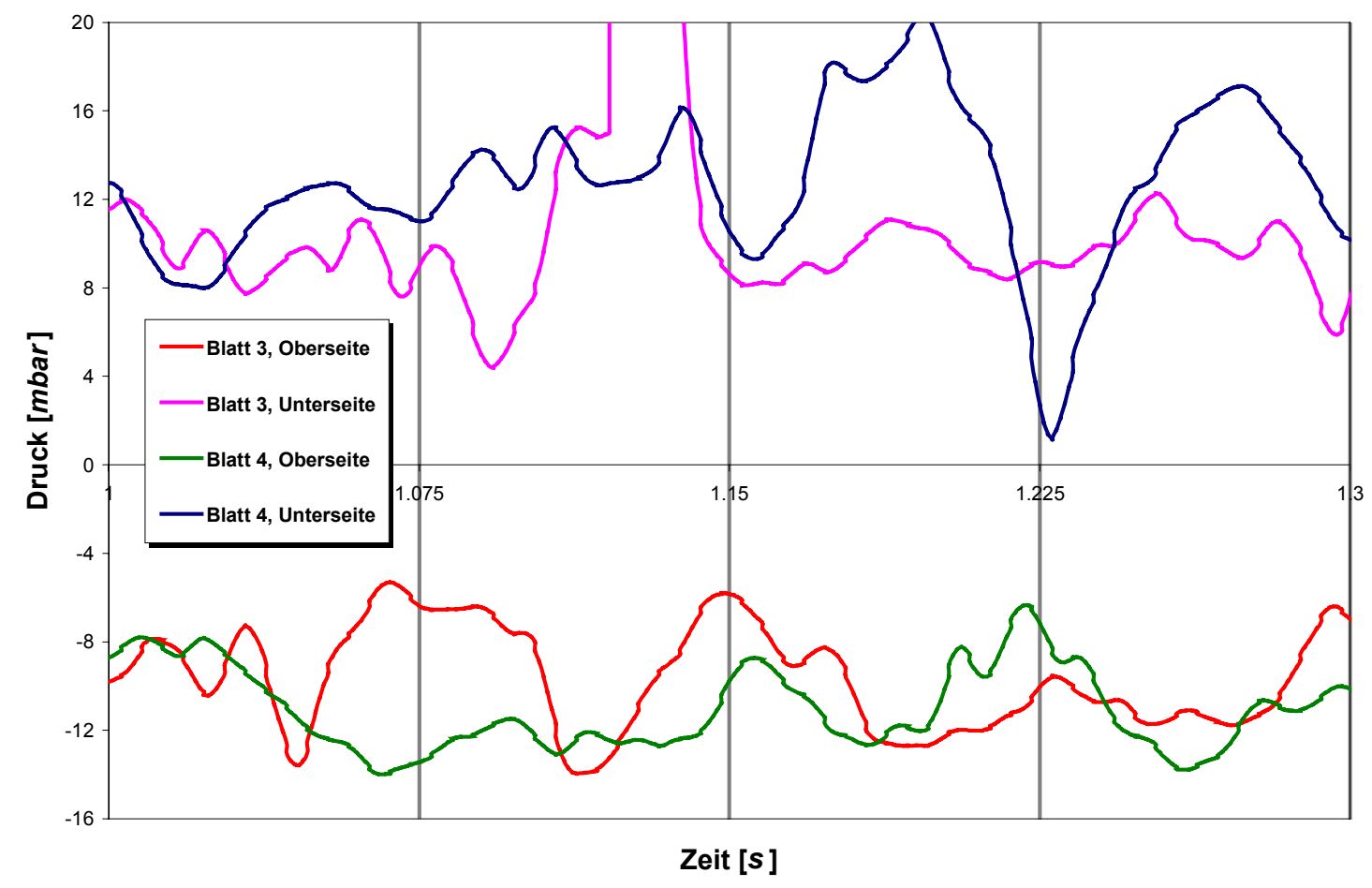

Abbildung 5.18: Druckverläufe im Experiment (4 Stromstörer)

In Abbildung 5.19 und Abbildung 5.20 werden die aus der Simulation ermittelten Drücke auf die vier Rührerblätter über der Zeit mit nur einem Stromstörer im Rührbehälter dargestellt.

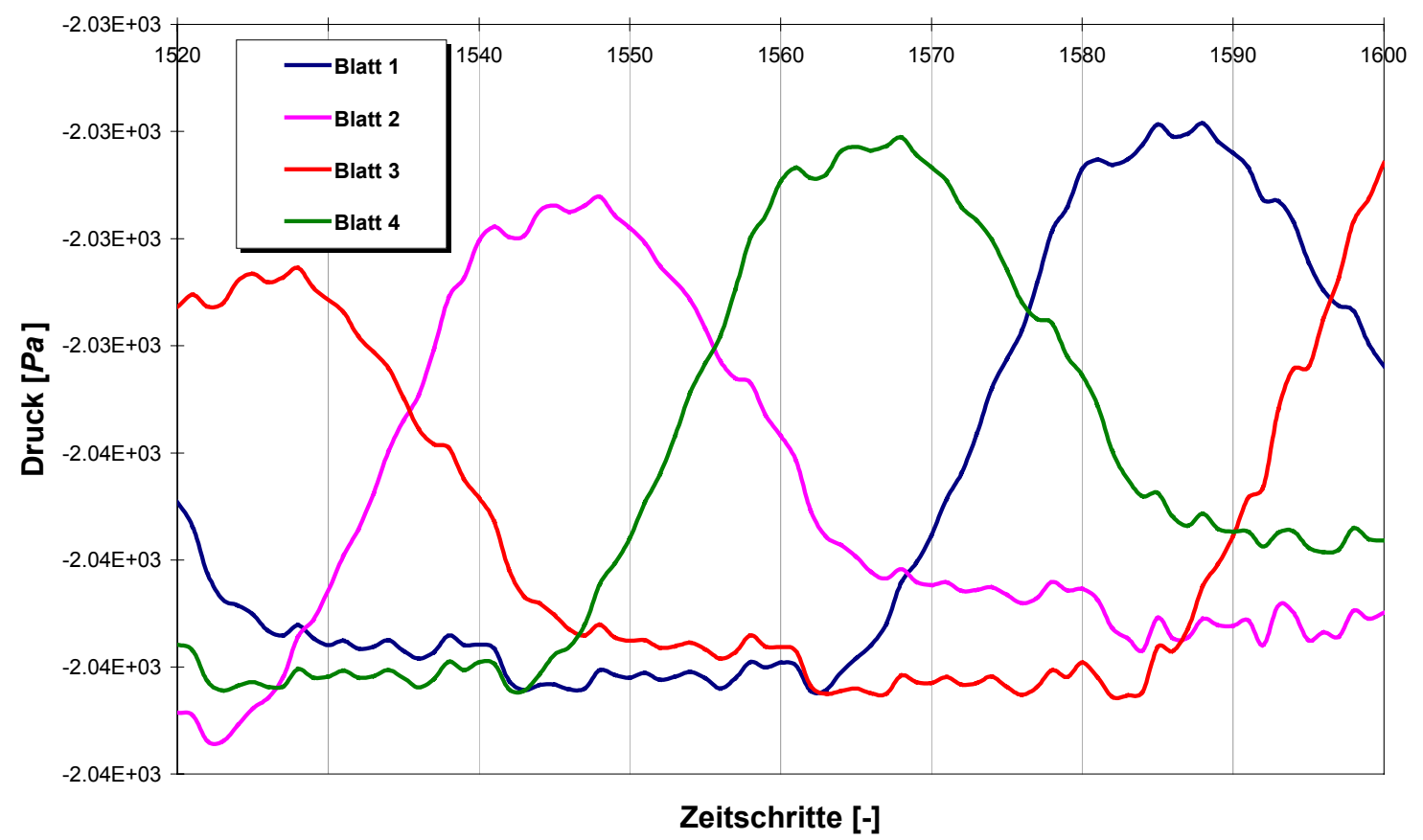

Abbildung 5.19: Oberseite der Rührerblätter (1 Stromstörer) 


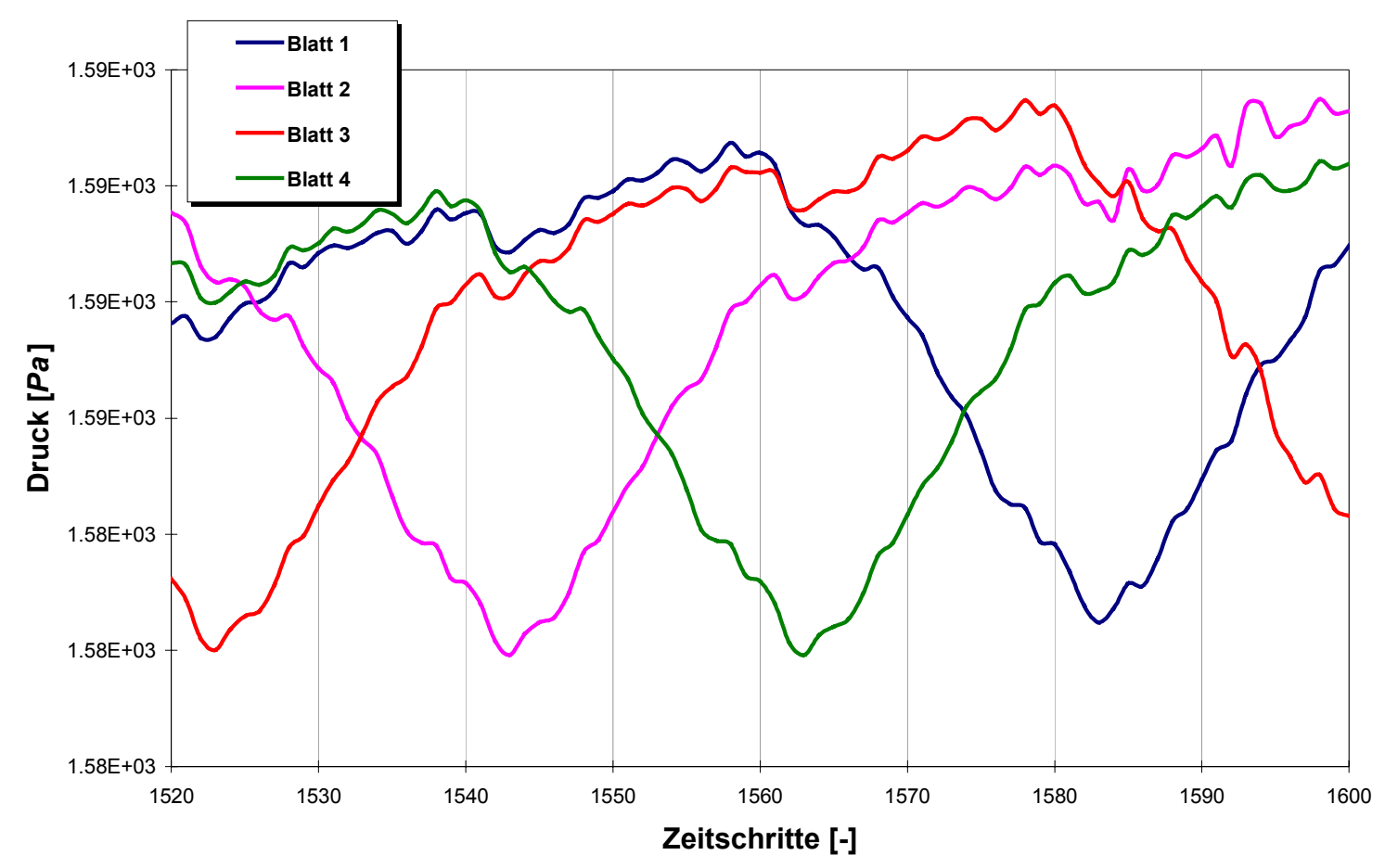

Abbildung 5.20: Unterseite der Rührerblätter (1 Stromstörer)

Der Druckverlauf an den einzelnen Blättern besitzt pro Umdrehung nur ein Extremum, kurz nachdem der Stromstörer passiert wird:

- $\quad$ Blatt 3: 1520 Zeitschritte

- $\quad$ Blatt 2: 1540 Zeitschritte

- $\quad$ Blatt 4: 1560 Zeitschritte

- $\quad$ Blatt 1: 1580 Zeitschritte

Auf die Oberseite der Rührerblätter (Saugseite) wirkt dabei ein Druckmaximum, auf die Unterseite (Druckseite) ein Druckminimum.

Aufgrund der fehlenden Kompensation der Impulse an gegenüberliegenden Rührerblättern bei unsymmetrischer Anordnung der Stromstörer kommt es zu einer Verstärkung der Anregung des Rührwerks. Im Resonanzfall ergibt sich somit ein größeres unterkritisches RMS-Amplitudenmaximum als beim Betrieb mit beispielsweise vier Stromstörern.

Für sämtliche aufgeführten Anordnungen wurden sowohl Simulationen als auch Experimente mit unterschiedlichen Drehzahlen durchgeführt. Es ergeben sich ähnliche Druckverläufe wie bei den aufgeführten Beispielen mit einer Drehzahl von 200U/min, jedoch mit unterschiedlich großen, von der Drehzahl abhängigen, Absolutwerten des Drucks. 


\subsection{Einfluss der Einspannsteifigkeit auf das Frequenzverhalten des Rührwerks}

Die Bewegungsbahn des fliegenden Rührwerks ist maßgeblich von der Einspannung des Rührwerks abhängig.

Wie aus Abbildung 5.21 deutlich zu erkennen ist, nehmen die kritische Drehzahl und die RMS-Amplitude bei weicherer Einspannsteifigkeit zwischen Rührwerk und Träger ab.

Bei gleicher Steifigkeit in $y$ - und $z$-Richtung (Einspannebene) bildet sich ein signifikantes RMS-Amplituden-Maximum im Bereich der kritischen Drehzahl aus. Beispiele hierfür sind:

- $\quad$ starre Einspannung

- Einspannung auf eine ebene Platte

- $\quad$ Einspannung auf einen Kugelboden

Bei vielen realen Lagerungen ist die Einspannsteifigkeit oft aus konstruktiven Gründen in $y$ - und $z$-Richtung unterschiedlich [120]. Ein Beispiel hierfür ist der Vierkantträger, der durch die Rührwerksbewegung sowohl auf Biegung (y-Richtung) als auch auf Torsion (z-Richtung) belastet wird. Die Torsionssteifigkeit des Vierkantträgers ist geringer als die Biegesteifigkeit, wodurch sich zwei unterschiedliche kritische Drehzahlen (700U/min und 900U/min) mit den jeweiligen Maxima der Schwingungsamplitude des Rührwerks ausbilden.

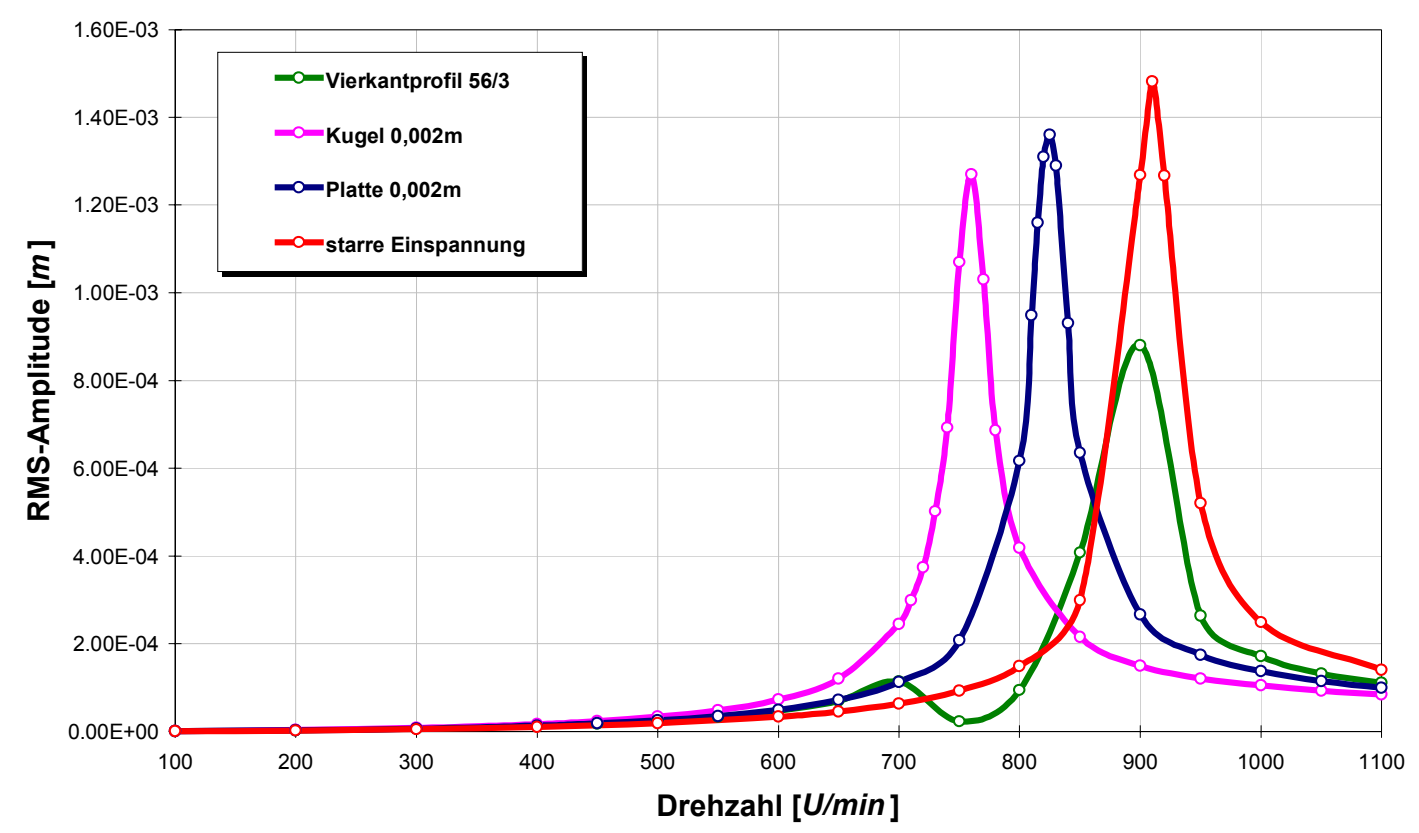

Abbildung 5.21: Vergleich unterschiedlicher Einspannverhältnisse 
In den folgenden Abbildungen 5.22 - 5.24 sind die Bewegungsbahnen (Trajektorien) des Rührwerks für unterschiedliche Einspannsteifigkeiten dargestellt. Es wird der unterkritische, der kritische und der überkritische Drehzahlbereich betrachtet. Die Achsen der Koordinatensysteme sind skaliert.

Bei ideal starrer Lagerung (linke Spalten) führt das Rührwerk über den gesamten Drehzahlbereich eine sogenannte Taumelbewegung aus, d.h. das Rührwerk beschreibt eine gleichmäßige Kreisbahn, deren Amplitude von der Drehzahl abhängt. Erwähnenswert ist hierbei der unterschiedliche Einlaufvorgang im unterkritischen und kritischen Drehzahlbereich. Während im unterkritischen Drehzahlbereich das Rührwerk erst nach mehreren Überschwinger auf eine quasistationäre Bahn einläuft, wird diese im überkritischen Bereich durch spiralförmige Steigerung der Auslenkung erreicht (siehe Abbildung 5.23 linke Spalte und Abbildung 5.24 linke Spalte)

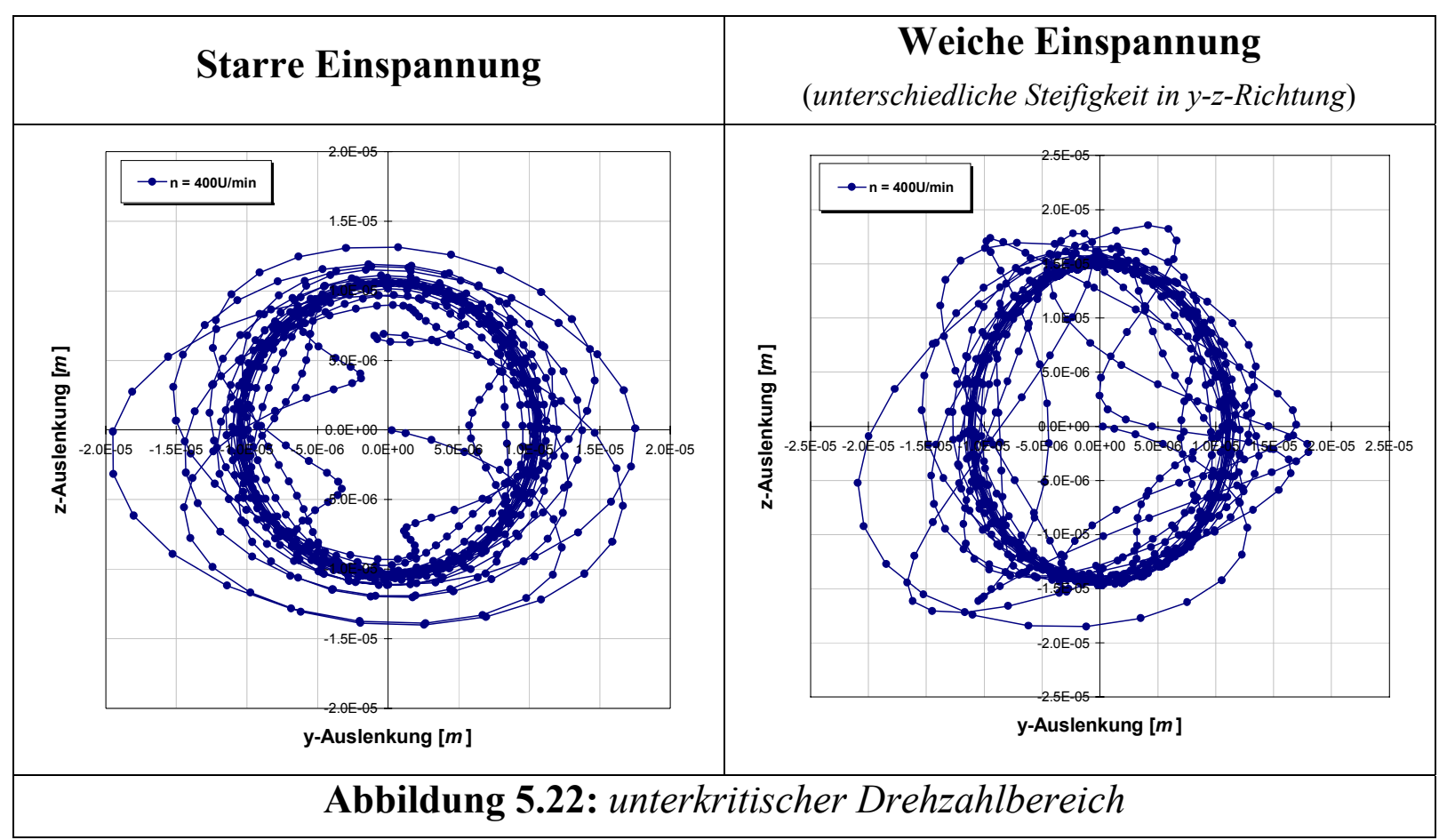


Bei weicher Einspannung und unterschiedlicher Steifigkeit in $y$ - und $z$-Richtung nimmt die Kreisbahn des Rührwerks bei Annäherung an die erste kritische Drehzahl (700U/min) zunehmend die Form einer schmalen Ellipse an. Hier weist die RMSAmplitude des Rührwerks auch das erste Maximum auf.

Die Ausrichtung der Ellipse beim Erreichen der zweiten kritischen Drehzahl (900U/min) ist von der Ellipse bei der ersten kritischen Drehzahl verschieden. Sie wandert in Richtung der Biegebeanspruchung ( $y$-Richtung) des Vierkantprofils. Das RMS-Amplituden-Maximum bei der zweiten kritischen Drehzahl ist wesentlich größer als bei der ersten kritischen Drehzahl. Das Bewegungsverhalten ist unmittelbar auf die Größe der Richtungsabhängigkeit der Einspannverhältnisse zurückzuführen und bei geringeren Unterschieden schwächer ausgeprägt.

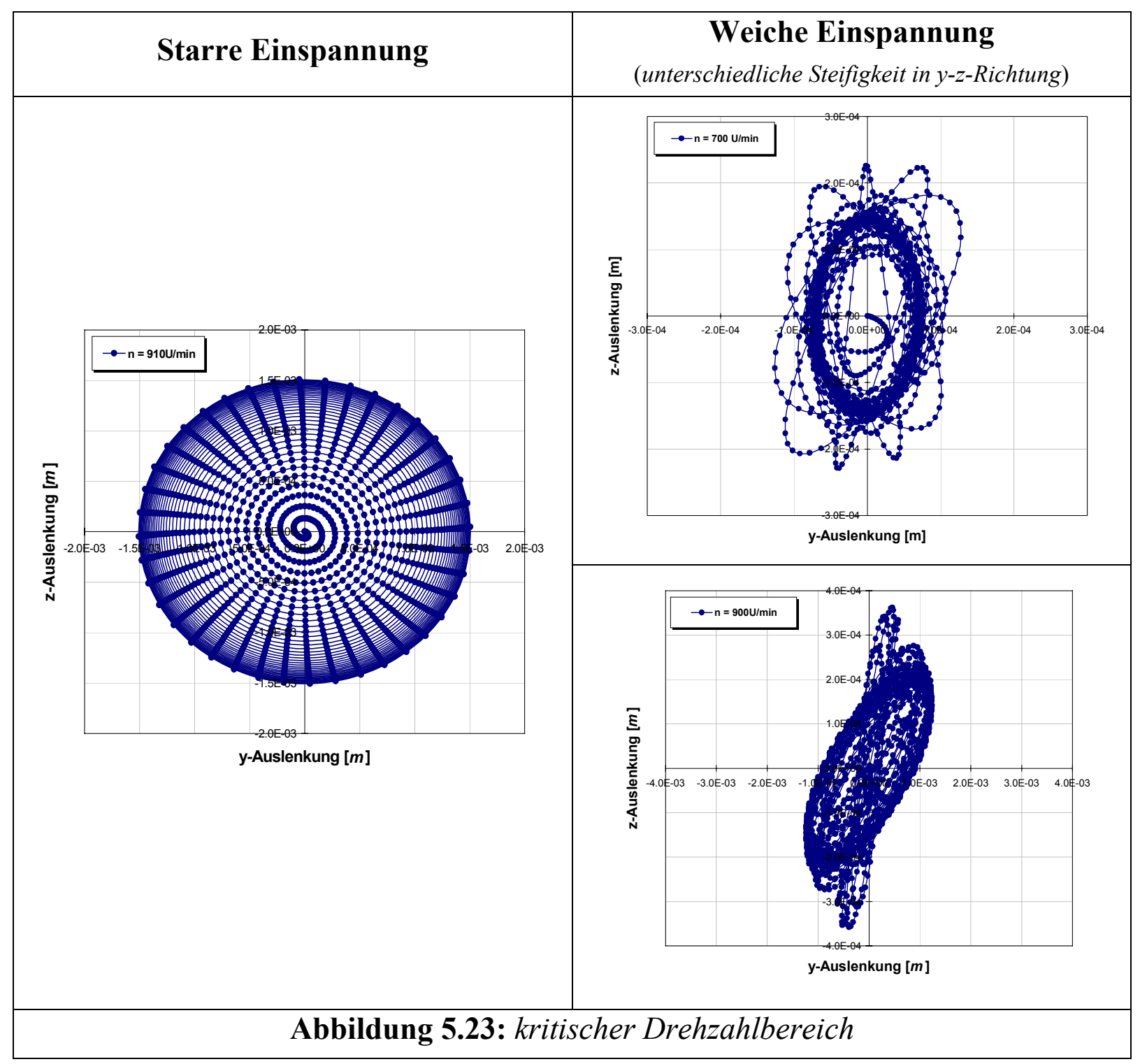


Nach Überschreiten der kritischen Drehzahl geht die Trajektorie des Rührwerks bei weicher Einspannung langsam wieder in die Form einer Kreisbahn mit geringerer Auslenkungsamplitude über. Es erfolgt die sogenannte Selbstzentrierung.

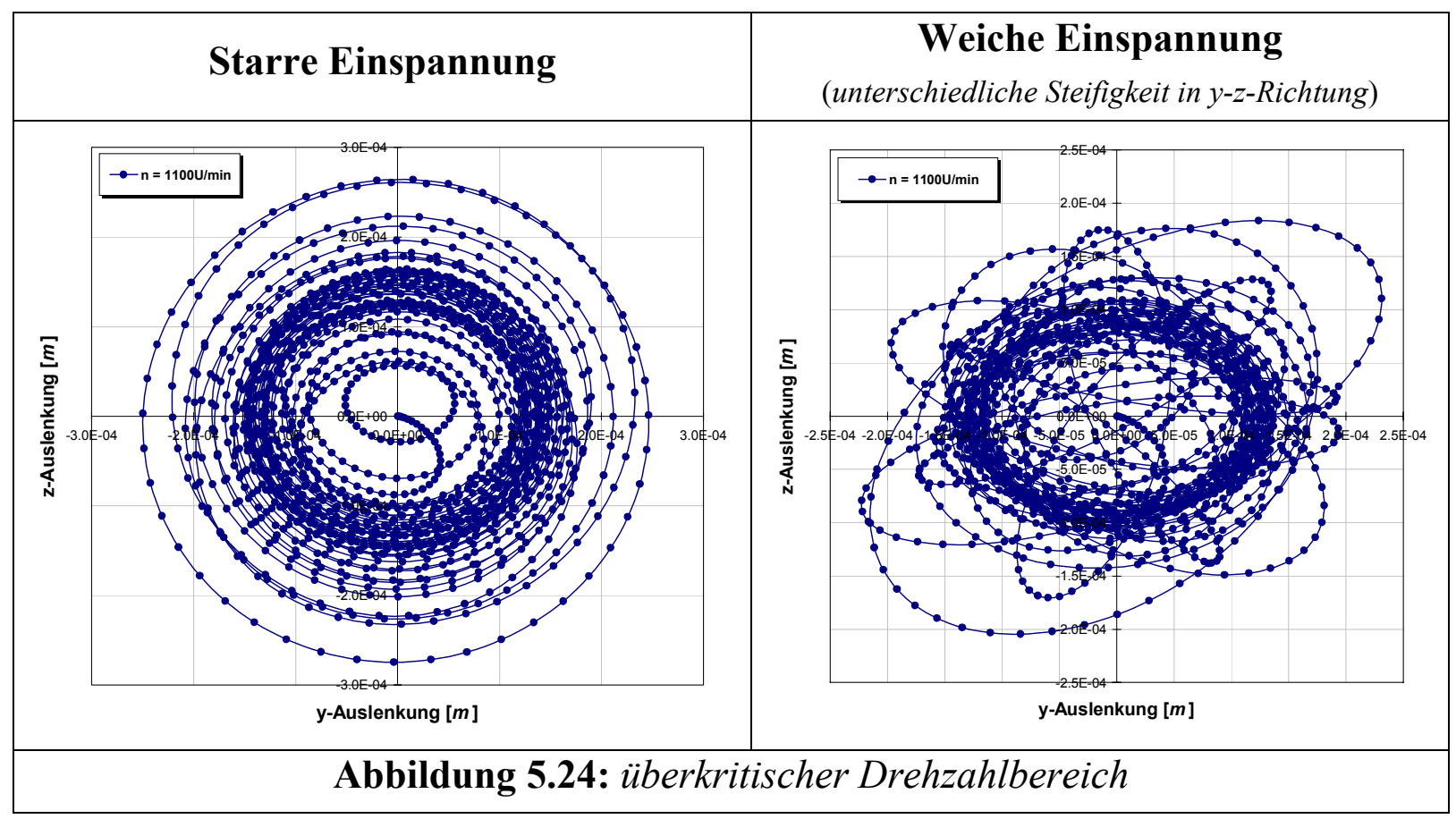




\section{Zusammenfassung und Ausblick}

Trotz der weitverbreiteten Anwendungsgebiete werden Rührwerke auch heutzutage noch weitgehend empirisch, mit aufwendigen experimentellen Verfahren, oder ungenauen analytischen Lösungen sicherheitstechnisch ausgelegt.

Unzureichende Berechnungsgrundlagen und Modelle sind oftmals die Ursache für Falschauslegungen und damit verbundene Schadensfälle; so auch bei der Auslegung von freifliegenden, schnelllaufenden Rührwerken in Fluiden, für deren korrekte sicherheitstechnische Auslegung die Eigenfrequenz, bzw. kritische Drehzahl $n_{k r i t}$ und die Auslenkungsamplituden wichtige Größen darstellen.

Das komplexe Wechselspiel zwischen der Mechanik des Rührwerks und den auf den Rührer einwirkenden fluiddynamischen Effekten, die ihrerseits wiederum Rückwirkungen auf die Mechanik des Rührwerks haben, lassen eine geschlossene analytische Lösung des dynamischen Verhaltens des Rührwerks nicht zu.

Desweiteren wird die Art der Rührwerksbefestigung am Behälterdach in den meisten analytischen Lösung nicht berücksichtigt. Hier wird oftmals von einer starren Einspannung zwischen Rührwerk und Einspannträger bzw. Behälterdach ausgegangen, die in der Realität, gerade bei großen Rührbehälterdurchmessern, selten zutrifft.

Kommerzielle FEM-Programme sind zum gegenwärtigen Zeitpunkt nicht in der Lage, voll fluid-struktur-gekoppelte Probleme bei praxisrelevanten Reynoldszahlen zu berechnen. Eine vollständige fluid-struktur-gekoppelte Simulation von FEM-Modell und CFD-Modell, die über den ständigen Austausch von Größen wie Kräften, Verschiebungen und Geschwindigkeiten zwischen den beiden Programmpaketen laufen müsste, ist sehr rechenzeit- und speicherplatzaufwendig sowie programmiertechnisch zu schwerfällig.

In dieser Arbeit wurde ein völlig neuer Weg beschritten. Ein kommerzieller CFDCode wird sowohl durch ein numerisches Ersatzmodell, und zur zusätzlichen Validierung, durch ein numerisches Balkenmodell für die Rührerdynamik erweitert (Fluid-Struktur-Kopplung). Das numerische Ersatzmodell erweist sich nach zahlreichen Untersuchungen dem komplexeren Balkenmodell bezüglich der Rechengenauigkeit als ebenbürtig, hat allerdings entscheidende Vorteile bezüglich Stabilität und Rechenzeit.

Das simulierte Strömungsfeld im Rührbehälter wird vollständig mit der Rührwerksstruktur gekoppelt. Somit können sämtliche zeitabhängigen fluiddynamischen Kräfte auf das Rührwerk übertragen werden. Für die 
Strömungsberechnung kommt ein Finite-Volumen-Verfahren mit verschiebbaren und deformierbaren Kontrollvolumina bei gleichzeitiger Anwendung der „sliding mesh“Methode zum Einsatz.

Mögliche weiche Einspannverhältnisse zwischen Rührwerk und Halterung werden über analytische Berechnungskonzepte in das numerische Ersatzmodell einbezogen.

Das in den CFD-Code implementierte numerische Ersatzmodell für die Rührerdynamik wurde mit experimentellen Ergebnissen im Leerlauf und Wasser verglichen und zeigte für unterschiedliche Rührwerke eine sehr gute Übereinstimmung. Hierzu wurden zahlreiche Parameterstudien mit Gitterzellenanzahlen, zeitlichen Diskretisierungsverfahren, Zeitschrittweiten, Iterationsanzahlen und Turbulenzmodellen durchgeführt.

Die beiden untersuchten Rührertypen „Schrägblattrührer“ und „,F1 ${ }^{\odot}$-Rührer“ zeigten bei einer Modellierung mit ca. 35000 Gitterzellen, dem $k-\varepsilon$ Turbulenzmodell, dem Newmark-Verfahren zur zeitlichen Diskretisierung der Struktur, 80 Zeitschritten pro Umdrehung und 10 Iterationen pro Zeitschritt stabile und genaue Simulationsergebnisse. Für genauere Untersuchungen der fluidinduzierten Anregung im unterkritischen Drehzahlbereich muss mit sogenannten Low-Re Turbulenzmodellen und wesentlich feineren Gittermodellen gearbeitet werden, was einen erheblichen Mehraufwand an Speicherkapazität und Rechenzeit mit sich zieht.

Mit dem entwickelten numerischen Berechnungsverfahren ist man somit in der Lage:

- die kritische Drehzahl von Rührwerken und zu vermeidende Betriebsbereiche und

- $\quad$ die drehzahlabhängigen Auslenkungsamplituden des Rührwerks über der Zeit für variierende Einspannverhältnisse, verschiedene Rührwerks- und Rührbehältergeometrien und unterschiedliche Rührmedien schon in der Planungsphase $\mathrm{zu}$ bestimmen.

Das Verfahren stellt im Vergleich zu experimentell basierten Auslegungen, eine kostengünstigere und verglichen mit halbempirischen Ansätzen eine genauere Auslegungsmethode mit breiterem Anwendungsspektrum dar. 
Zukünftige Arbeiten mit dem entwickelten Berechnungsprogramm könnten mit noch feineren Gittern und mit Hilfe von „Large-Eddy“ oder mit erst kürzlich entwickelten „Detached-Eddy“-Ansätzen, welche eine Kombination aus einer „Large-Eddy“ Simulation und einem statistischen Modell darstellen, simuliert werden. Dadurch kann ein noch genauerer Einblick in das Strömungsfeld und beispielsweise in die fluidinduzierte Anregung durch Wirbelablösungen bei Rührwerken gewonnen werden. Aufgrund der fortschreitenden Rechnertechnologie mit schnelleren Prozessoren und größeren Speicherbausteinen werden derartige Simulationen auch bei Rührermodellen in einem kleineren Zeitfenster und auf handelsüblichen PC's möglich sein.

Weitere denkbare Untersuchungen mit dem entwickelten numerischen Berechnungsprogramm sind Variationen des Rührmediums bis in den nichtNewtonschen (hochviskosen) Bereich, Simulationen mit mehrstufigen Rührwerken und mehrphasige Berechnungen mit z.B. freien Oberflächen, etwa zur Untersuchung von Durchtrittsbetrieben. 


\section{Literaturverzeichnis}

[1] Angst, R.; Kraume, M.:

Grid and model dependency of the solid/liquid two phase flow CFD

simulations of stirred vessels

Preprints of the $11^{\text {th }}$ European Conference on Mixing, $14^{\text {th }}$ to $17^{\text {th }}$ October

2003, Bamberg, Germany, $637^{\text {th }}$ Event of the European Federation of

Chemical Engineering

[2] Bakker, A.; Laroche, R.D.; Wang, M.H.; Calabrese, R.V.:

Sliding Mesh Simulation of Laminar Flow in Stirred Tanks

Transactions of the Institution of Chemical Engineers, Vol. 75 (A), 1997

[3] Bartels, C.; Breuer, M.; Durst, F.:

Comparison between Direct Numerical Simulation and $k$ - $\varepsilon$ Prediction of the

Flow in a Vessel Stirres by a Rushton Turbine

Proceedings of the $10^{\text {th }}$ European Conference on Mixing, Delft, The Netherlands, July 2-5, 2000

[4] Bäßler, O.:

Der Einfluss von Wälzlagern auf die Biegeschwingungen von Wellen

Konstruktion 15/3, 1963

[5] Bathe, H.-J.:

Finite Element Methoden

Springer Verlag, Berlin, Heidelberg, New York, 1986

[6] Beitz, W.; Grote, K.-H.:

Dubbel - Taschenbuch für den Maschinenbau

20. Auflage, Springer Verlag, Berlin, Heidelberg, New York, 2001

[7] Berger, T.:

Mischzeitberechnungen in Rührbehältern mit CFX-4

Diplomarbeit AEA Technology, TU München, 1998 
[8] Berger, T.; Strohmeier, K.:

Numerische Strömungssimulation verschiedener Rührwerke zur Bestimmung von Strömungskräften, Leistungsaufnahme und Mischzeiten

Kurzfassung der Referate der internen Arbeitssitzung der GVCFachausschüsse „CFD Computational Fluid Dynamics“, „Mehrphasenströmungen“" und ADMIRE, 28.02. - 01.03.2000, Bamberg

[9] Berger, T.; Strohmeier, K.:

Numerical Eigenfrequency Determination of Stirrers Considering FluidStructure Interaction

ASME PVP Vol.414-2, Emerging Technologies in Fluids, Structures and Fluid/Structure Interactions, Seattle, WA, USA, July $23^{\text {rd }}-27^{\text {th }} 2000$

[10] Berger, T.; Strohmeier, K.:

CFD Simulation von Fest-/Flüssigströmungen in Rührbehältern

Kurzfassung der Referate der internen Arbeitssitzung der GVCFachausschüsse „CFD Computational Fluid Dynamics“ und „Rheologie“, 21.02. - 23.02.2001, Weimar

[11] Berger, T.; Strohmeier, K.:

Bestimmung der kritischen Drehzahl von Rührern unter Berücksichtigung der Fluid-Struktur-Wechselwirkung

Kurzfassung der Referate der internen Arbeitssitzung der GVCFachausschüsse „Wärme- und Stoffübertragung“ und „CFD Computational Fluid Dynamics“, 04.03. - 06.03.2002, Weimar

[12] Berger, T.; Eck1, B.; Strohmeier, K.:

Fluid Structure Interaction of Stirrers in Mixing Vessels - Part II: Fully Coupled Simulation

ASME PVP Vol. 446-1, Emerging Technologies in Fluids, Structures and Fluid/Structure Interactions, Vancouver, BC, Canada, August $4^{\text {th }}-8^{\text {th }} 2002$ 
[13] Berger, T.; Strohmeier, K.:

Entwicklung eines numerischen Berechnungsverfahrens für

Rührwerksschwingungen

Kurzfassung der Referate der internen Arbeitssitzung der GVCFachausschüsse „Mischvorgänge“ und „CFD Computational Fluid Dynamics“, 31.03. - 02.04.2003, Berlin

[14] Berger, T.; Strohmeier, K.:

Numerical simulation of stirrer oscillations in different fluids (fully coupled fluid-structure-interaction)

Vortrag 21.5.2003, 27 $7^{\text {th }}$ International Exhibition on Chemical Engineering, Environmental Protection and Biotechnology. Frankfurt am Main, Germany, $19^{\text {th }}-24^{\text {th }}$ May 2003. In: ACHEMA $200327^{\text {th }}$ International ExhibitionCongress on Chemical Engineering, Environmental Protection and Biotechnology. Abstracts of the lecture groups

[15] Berger, T.; Strohmeier, K.:

Numerical Simulation of Stirrer Oscillations in Consideration of FluidStrucuture-Interaction and Flexible Restraint Systems

ASME PVP Vol. 460, Emerging Technologies in Fluids, Structures and Fluid/Structure Interactions, Cleveland, Ohio, July $20^{\text {th }}-24^{\text {th }} 2003$

[16] Berger, T.; Strohmeier, K.:

Structural dynamic analysis of stirrers with a fluid-structure-coupling algorithm in CFD

Preprints of the $11^{\text {th }}$ European Conference on Mixing, $14^{\text {th }}$ to $17^{\text {th }}$ October 2003, Bamberg, Germany, 637 ${ }^{\text {th }}$ Event of the European Federation of Chemical Engineering

[17] Berger, T.; Fischer, M.; Strohmeier, K.:

Fluid-Structure Interaction of Stirrers in Mixing Vessels

Journal of Pressure Vessels Technology, Transactions of the ASME, Nov. 2003 
[18] Birthig, A.; Lauschke, G.; Schierholz, W.F.; Beck, D.; Maul, C.; Gilbert, N.; Wagner, H.-G.; Werninger, C.Y:

CFD in der chemischen Verfahrenstechnik aus industrieller Sicht

Chemie Ingenieur Technik (72), No. 3, 2000

[19] Bradshaw, P.:

Turbulence

Springer Verlag, Berlin, Heidelberg, New York, 1976

[20] Bremer, H.; Pfeiffer, F.:

Elastische Mehrkörpersysteme

B.G. Teubner, 1992

[21] Burger, S.:

Einfluss dynamischer Krafteinwirkungen auf die Biegeschwingungen von Rührwellen

Chemie Ingenieur Technik 43, 1971

[22] Burger, S.:

Beitrag zur Berechnung von schnelllaufenden Rührwerken

Dissertation TU München, 1975

[23] Cebeci, T.; Smith, A.M.O.:

Analysis of Turbulent Boundary Layers

Academic Press Inc., New York, 1974

[24] CFX Short Course

Advanced Turbulence Modelling

Garmisch-Partenkirchen, Germany, ANSYS Germany GmbH, 4.-6- November 2003

[25] DANTEC Dynamics GmbH:

Flow Measurement - Laser-Doppler-Anemometry

Betriebsanleitung 
[26] Decker, S.; Sommerfeld, M.:

Numerical Calculations of Two-Phase Flows in Agitated Vessels using the Euler/Langrange Approach

Proceedings of the ASME FEDSM'00 11154, June $11^{\text {th }}-15^{\text {th }}, 2000$, Boston Massachusetts

[27] Demirdzic, I.; Peric, M.:

Space Conservation Law in Finite Volume Calculations of Fluid Flow

Int. Journal for numerical Methods in Fluids, Vol. 8, 1988

[28] Durst, F.; Melling, A.; Whitelaw, J.H.:

Theorie und Praxis der Laser-Doppler-Anemometrie

G. Braun Verlag, Karlsruhe, 1987

[29] Enger, S.; Breuer, M.; Durst, F.:

Experimentelle und numerische Untersuchungen von mehrphasigen Strömungen in Rührreaktoren (Numerische Arbeiten)

3. Fortschrittsbericht zum Schwerpunktprogramm Analyse, Modellbildung und Berechnung mehrphasiger Strömungen

[30] ERCOFTAC Special Interest Group on „Quality and Trust in Industrial CFD“ Editors: Casey, M., Wintergerste, T.

Best Practice Guidelines

Version 1.0, January 2000

[31] Ertem-Müller, S.; Schäfer, M.:

DNS and LES of the Turbulent Flow in a Rushton-Turbine

Kurzfassung der Referate der internen Arbeitssitzung der GVCFachausschüsse „Mischvorgänge“ und „CFD Computational Fluid Dynamics“, 31.03. - 02.04.2003, Berlin

[32] Fasano, J.B.; Miller, J.L.; Pasley, S.A.:

Consider mechanical design of agitators

Chemical Engineering Progress, August 1995, S.60-71 
[33] Ferziger, J.H.; Peric, M.:

Computational Methods for Fluid Dynamics

Springer Verlag, Berlin, Heidelberg, New York, 1999

[34] Fischer, M.; Strohmeier, K.:

The Use of Algebraic Computer Systems to find Analytical Solutions in Continuum Mechanics and Applications to Fluid-Structure Interaction Problems

ASME PVP Vol. 414-2, Emerging Technologies in Fluids, Structures and Fluid/Structure Interactions, Seattle, WA, USA, July $23^{\text {rd }}-27^{\text {th }} 2000$

[35] Fischer, M.:

Dreidimensionale numerische Simulation strömungsinduzierter Rohrbündelschwingungen

Dissertation TU München, 2001

[36] Gasch, R.:

Unwucht-erzwungene Schwingungen und Stabilität von Turbinenläufern

Konstruktion im Maschinen-Apparate-und Gerätebau, 25. Jahrgang, Heft 5, Mai 1973

[37] Gasch, R.; Pfützner, H.:

Rotordynamik - Eine Einführung

Springer Verlag, Berlin, Heidelberg, New York, 1975

[38] Gasch, R.; Knothe, K.:

Strukturdynamik, Band 1: Diskrete Systeme

Springer Verlag, Berlin, Heidelberg, New York, 1987

[39] Gasch, R.; Knothe, K.:

Strukturdynamik, Band 2: Kontinua und ihre Diskretisierung

Springer Verlag, Berlin, Heidelberg, New York, 1989 
[40] Geisler, R.:

Fluiddynamik und Leistungseintrag in turbulent gerührten Suspensionen

Dissertation, TU München, 1991

[41] Geisler, R.:

Einsatzbereiche von Rühr- und Knetreaktoren für verfahrenstechnische Grundaufgaben

Kurzfassung der Referate der internen Arbeitssitzung der GVCFachausschüsse „Mischvorgänge“ und „CFD Computational Fluid Dynamics“, 31.03. - 02.04.2003, Berlin

[42] Hagedorn, P.:

Technische Schwingungslehre, Band 2

Springer Verlag, Berlin, Heidelberg, New York, 1989

[43] Höhnel, L.:

Diplomarbeit, Technische Universität Dresden, 1974

[44] Hortmann, M.:

Stationäre und instationäre Berechnung von Rührkesseln -Theorie und Praxis-

Kurzfassung der Referate der internen Arbeitssitzung der GVCFachausschüsse „Mischvorgänge“ und „CFD Computational Fluid Dynamics“, 31.03. - 02.04.2003, Berlin

[45] Jamamoto, T.:

On Critical Speeds of a Shaft Supported by a Ball Bearing

Transactions of the ASME, Journal Of Applied Mechanics, 1959

[46] Jones, W.; Launder, B.:

The Prediction and Laminarization with a Two-Equation Model of Turbulence International Journal of Heat and Mass Transfer, Vol. 15, 1972 
[47] Jürgler, R:

Maschinendynamik

VDI-Verlag, 1996

[48] Kantorowitsch, S. B.:

Chemiemaschinen: Zentrifugen - Filter-Drehöfen

VEB Verlag Technik, Berlin 1970

[49] Kassera, V.:

Entwicklung eines numerischen Berechnungsverfahrens für Schwingungen querangeströmter Rührbündel

Dissertation Lehrstuhl für Apparate- und Anlagenbau, TU München, 1997

[50] Kassera, V.; Kraume, M.; Berger, T.:

CFD Simulation of Liquid-Particle Flows in Mixing Vessels

ASME PVP Vol. 414-2, Emerging Technologies in Fluids, Structures and

Fluid/Structure Interactions, Seattle, WA, USA, July $23^{\text {rd }}-27^{\text {th }} 2000$

[51] Kipke, K.:

Hydraulische Belastungen von Kesselreaktoren

Chem.-Ing.-Tech. 56, S. 733-739, 1984

[52] Kipke, K.:

Auslegung von Industrie Rührwerken

Chem. Ing. Tech. 57, S. 813 - 823, 1985

[53] Kneule, F.:

Praxis der Verfahrenstechnik - Rühren

DECHEMA Deutsche Gesellschaft für Chemisches Apparatewesen, Chemische Technik und Biotechnologie e.V., Frankfurt, 1986

[54] Knoch, A:

Biegebeanspruchung von Rühraggregaten

Dissertation Universität GH Paderborn, Shaker Verlag, 2000 
[55] Knudson W.C.; Surya Kumar, G.V.:

Explicit Time Integration Operators for Nonlinear Structural Dynamics

Mitteilungen 51/1979 des Sonderforschungsbereichs 64, Universität Stuttgart, 1979

[56] Kohnen, C.; Bohnet, M.:

Messung und Simulation der Fluidströmung in gerührten Fest/FlüssigSuspensionen

Chemie Ingenieur Technik (72), No. 6, 2000

[57] Kraume, M.:

Mischen und Rühren: Grundlagen und moderne Verfahren

WILEY-VCH, 2002

[58] Launder, B.E.; Spalding, D.B.:

The Numerical Computation of Turbulent Flows

Comp. Meth. Appl. Mech. Eng., Vol. 3, 1974

[59] Launder, B.E.; Sharma, B.I.:

Application of the Energy Dissipation Model of Turbulence to Calculation of Flow Near a Spinning Disc

Letters on Heat and Mass Transfer, Vol. 15, 1974

[60] Launder, B.E.; Reynolds, W.C.; Rodi, W.; Mathieu, J.; Jeandel, D.:

Turbulence Models and their Applications

Direction des Etudes et Recherches d'Electricite de France, Editions Eyrolles, Paris, 1984

[61] Liepe, F.; Meusel, W.; Möckel, H.O.; Platzer, B.; Weißgräber, H.:

Verfahrenstechnische Berechnungsmethoden, Teil 4: Stoffvereinigen in fluiden Phasen - Ausrüstungen und ihre Berechnungen

VCH Verlagsgesellschaft, Weinheim, 1988 
[62] Magnus, K.:

Schwingungen

B.G. Teubner, Stuttgart, 1976

[63] Manninen, M.; Syrjänen, J.:

Modelling Turbulent Flow in Stirred Tanks

CFX-Update Nr. 16, 1998

[64] MARC/MENTAT:

Dynamic Analysis with MARC

Unterlagen zur technischen Schulung MTU: 99-300-1, München, 25.26.02.1999

[65] MARC/MENTAT:

Dokumentation, User's Guide, Volume A, Theory and User

[66] Melzer, B.:

Theoretische und experimentelle Untersuchung des dynamischen Verhaltens von Rührerwellen

Dissertation, TU München, 1970

[67] Meyberg, K.; Vachenauer, P.:

Höhere Mathematik 2

Springer Verlag, Berlin, Heidelberg, New York, 1990

[68] Michelassi, V.; Rodi, W.; Zhu, J.:

Testing of Low-Reynolds Number $k$ - $\varepsilon$ Turbulence Models based on Direct Simulation Data

AIAA Journal, vol. 31, no. 9, 1993

[69] Milchenko, A.J.:

Conditions for High-Speed Mixer Shafts

Chemical and Petroleum Engineering 1965/1966 
[70] Mooser, A.:

Numerische Simulation von Zweiphasenströmungen in Rührbehältern

Konstruktive Semesterarbeit am Lehrstuhl für Apparate- und Anlagenbau TU München, 2000

[71] Müller, Chr.:

Modellierung und numerische Simulation von Einphasenströmungen in Rührbehältern

Konstruktive Semesterarbeit am Lehrstuhl für Apparate- und Anlagenbau, TU München, 2001

[72] Newmark, N.M.:

A Method of Computation for Structural Dynamics

ASCE, Journal of the Engineering Mechanics Division, Band 85

[73] Noll, B.:

Numerische Strömungsmechanik

Springer Verlag, Berlin, Heidelberg, New York, 1993

[74] Orszag, S.A.:

Introduction to Renormalization Group Modelling of Turbulence

In: Simulation and Modelling of Turbulent Flows

Ed Thomas Gatski, M.Yousuff Hussaini, John L. Lumley, New York, Oxford, Oxford University Press, 1996

[75] Pahl, M.; Habermann, R..; Wittig, A.:

Mischen, Rühren, Kneten und Dosieren

Chemie Ingenieur Technik (72), No.12, 2000

[76] Paszek, E.:

The effect of liquid media on agitator shaft vibration

International Chemical Engineering, 14, Nr. 3, S. 451- 455, 1974 
[77] Patankar, S.:

Numerical Heat Transfer and Fluid Flow

Hemisphere Publishing, 1980

[78] Patankar, S.V.; Spalding, D.B.:

A Calculation Procedure for Heat, Mass and Momentum Transfer in ThreeDimensional Parabolic Flow

Int. Journal. Heat and Mass Transfer, Vol.15, 1972

[79] Perret:

Wälzlager als Schwingungserreger

Werkstatttechnik und Maschinenbau 40/1, 1950

[80] Pfeiffer, F.:

Einführung in die Dynamik

B.G. Teubner, Stuttgart, 1992

[81] Pollard, G.J.:

Hydraulic Bending Loads in Mixer Shafts

$4^{\text {th }}$ European Conference on Mixing, 27. - 29. April 1982, Paper K3

[82] Ramsey, W.D.; Zoller, G.C.:

How the Design of Shafts, Seals and Impellers Affects Agitator Performance Chem. Eng., August 1976

[83] Rank, E.:

Grundlagen und Anwendungen von Finite-Elemente-Verfahren in der Strukturmechanik

KONWIHR - Kurzlehrgang „Numerische Methoden zur Lösung von Ingenieurproblemen in der Strömungs- und Strukturmechanik“, 18. - 21. Februar 2002, München 
[84] Reichel, C.; Berger, T., Strohmeier, K.:

Numerical Calculation of Fluid-Forces in Different Types of StirringApparatus with CFD

ASME PVP Vol.414-2, Emerging Technologies in Fluids, Structures and Fluid/Structure Interactions, Seattle, WA, USA, July $23^{\text {rd }}-27^{\text {th }} 2000$

[85] Rodi, W.:

Turbulence Models and their Application in Hydraulics - A State of the Art Review

Habilitation Thesis, University of Karlsruhe, 1980

[86] Rodi, W.:

Turbulence Models and their Applications in Hydraulics

International Association of Hydraulic Research Publication, Delft, 1980

[87] Schäfer M.:

Anwendung der Laser-Doppler-Anemometrie in der Rührtechnik

Kurzlehrgang HDA-LDA, Lehrstuhl für Strömungsmechanik, FriedrichAlexander-Universität Erlangen-Nürnberg, 1998

[88] Schäfer, M.; Yianneskis, M.; Wächter, P.; Durst, F.:

Trailing Vortices Around a $45^{\circ}$ Pitched-Blade Impeller

AIChE Journal, Vol. 44, Nr. 6, 1998

[89] Schäfer, M.:

Numerik im Maschinenbau

Springer Verlag, Berlin, Heidelberg, New York, 1999

[90] Schäfer, M.:

Coupled Fluid-Solid Problems: Survey on Numerical Approaches and Applications

Fluid-Struktur-Wechselwirkung, Tagung Wiesloch 11./12. Juni 2002, VDI Berichte 1682 
[91] Schilcher, S.:

Numerische Simulation des Strömungsfelds eines Schrägblatt- und $F 1^{{ }^{\circ}}$ Rührers und Vergleich mit Messergebnissen

Konstruktive Semesterarbeit am Lehrstuhl für Apparate- und Anlagenbau, TU München, 2003

[92] Schilling R.:

Modellbildung und Simulation

Skript zur Vorlesung, Lehrstuhl für hydraulische Maschinen, TU München

[93] Schlichting, H.; Gersten, K.:

Grenzschicht-Theorie

Springer Verlag, Berlin, Heidelberg, New York, 1997

[94] Schmid, P.:

Strömungstechnisches Versuchswesen

Skript zur Vorlesung, Lehrstuhl für Fluidmechanik, TU München

[95] Schnell, W.; Gross, D.; Hauger, W.:

Technische Mechanik, Band 2, Elastostatik

6.Auflage, Springer Verlag, Berlin, Heidelberg, New York, 1999

[96] Schnell, W.; Gross, D.; Hauger, W.:

Technische Mechanik, Band 3, Kinetik

6.Auflage, Springer Verlag, Berlin, Heidelberg, New York, 1999

[97] Schönung, B.E.:

Numerische Strömungsmechanik

Springer Verlag, Berlin, Heidelberg, New York, 1990

[98] SENSOPLAN GmbH.

Neue Messverfahren setzen neue Maßstäbe in der Drehmomentmesstechnik Antriebstechnik, 35, 1996 
[99] SKF Wälzlager Katalog

Carl Gerber Verlag, 1990

[100] Smith, G.D.:

Numerische Lösung von partiellen Differentialgleichungen

Vieweg Verlag, Braunschweig, 1970

[101] Solver Manual zur Software CFX-4.4

AEA Technology, Oxfordshire, 1999

[102] Sommerfeld, M.; Decker, S.:

State of the Art and Future Trends in CFD Simulation of Stirred Vessels

Preprints of the $11^{\text {th }}$ European Conference on Mixing, $14^{\text {th }}$ to $17^{\text {th }}$ October

2003, Bamberg, Germany, $637^{\text {th }}$ Event of the European Federation of

Chemical Engineering

[103] Stelzer, E.:

Fehler an Rühranlagen

Kurzfassung der Referate der internen Arbeitssitzung der GVC-

Fachausschüsse „Mischvorgänge“ und „CFD Computational Fluid Dynamics“, 31.03. - 02.04.2003, Berlin

[104] Strohmeier, K.; Hölzl, R.:

Vibrational Damage to a Bio-Reactor

Chemical Engineering and Technology, Bd. 21, Nr. 4, 1998

[105] Strohmeier, K.:

Schwingungsschaden an einem Bio-Reaktor

Technische Überwachung, Bd. 37, Nr. 11/12, 1996

[106] Strohmeier, K.:

Komponenten des Anlagenbaus

Skript zur Vorlesung, Lehrstuhl für Apparate- und Anlagenbau, TU München 
[107] Strohmeier, K.:

Sicherheits-Simulationen in Rohrleitungssystemen

Skript zur Vorlesung, Lehrstuhl für Apparate- und Anlagenbau, TU München

[108] Todtenhaupt, P.; Todtenhaupt; E.; Müller, W.; u.a.:

Handbuch der Rührtechnik - Grundlagen, Auswahlkriterien, Anwendung

EKATO Rühr- und Mischtechnik GmbH, Schopfheim 2000

[109] Traupel, W.:

Thermische Turbomaschinen

Springer Verlag, Berlin, Heidelberg, New York, 1968

[110] Truckenbrodt, E.:

Fluidmechanik

Springer Verlag, Berlin, Heidelberg, New York, 1989

[111] Ulbrich, H.:

Dynamik und Regelung von Rotorsystemen

Fortschrittsberichte VDI Reihe 11 Nr.86, 1986

[112] Ulbrich, H.:

Maschinendynamik

B.G. Teubner, Stuttgart, 1996

[113] Van Doormaal, J.P.; Raithby, G.D.:

Enhancements of the SIMPLE Method for Predicting Incompressible Fluid Flows

Numerical Heat Transfer, Vol. 7, 1984

[114] VDI-Richtlinien:

Hinweise zur Messung und Interpretation der Schwingungen von Maschinen Schwingungsbilder für Anregungen aus Unwuchten. Montagefehlern, Lagerungsstörungen und Schäden an rotierenden Bauteilen 
VDI 3839, Blatt2, Mai 2003, VDI Handbuch Schwingungstechnik, Beuth Verlag, Berlin, Wien, Zürich

[115] Wächter, K.:

Konstruktionslehre für Maschineningenieure

Grundlagen, Konstruktions- und Antriebselemente

VEB Verlag Technik, Berlin, 1987

[116] Weiß, H.-J.; Krebs, R.:

Dynamische Belastung von Rührreaktoren

Hydrodynamik - Spannungsberechnung - Modalanalyse

EKATO, Rühr- und Mischtechnik GmbH, Schopfheim

[117] Wilcox, D.C.:

Turbulence Modelling for CFD

$2^{\text {nd }}$ Edition, DCW Industries, Inc., La Canada, California, 1998

[118] Wittenburg, J.:

Schwingungslehre, Theorie und Anwendungen

Springer Verlag, Berlin, Heidelberg, New York, 1996

[119] Yakhot V.; Orszag, S. A.:

Renormalization Group Analysis of Turbulence: 1. Basic Theory

Journal of Scientific Computing, Vol. 1, 1986

[120] Ziegler, G.:

Maschinendynamik

Auflage, Carl Hanser Verlag, München, Wien, 1977

[121] Zlokarnik, M.:

Rührtechnik - Theorie und Praxis

Springer Verlag, Berlin, Heidelberg, New York, 1999 
